\title{
LARGE SCALE DATA ANALYSIS FOR COMPUTATIONAL BIOCHEMISTRY
}

\author{
by \\ Xuemei Luo
}

\begin{abstract}
A thesis submitted to the Faculty of Graduate and Postdoctoral Affairs

in partial fulfillment of

the requirements for the degree of

DOCTOR OF PHILOSOPHY

School of Computer Science

at

CARLETON UNIVERSITY
\end{abstract}

Ottawa, Ontario

September, 2011

(C) Copyright by Xuemei Luo, 2011 
Library and Archives Canada

Published Heritage

Branch

395 Wellington Street Ottawa ON K1A ON4 Canada
Bibliothèque et

Archives Canada

Direction du

Patrimoine de l'édition

395 , rue Wellington

Ottawa ON K1A ON4

Canada
Your file Votre référence

ISBN: 978-0-494-83209-7

Our file Notre référence

ISBN: 978-0-494-83209-7
NOTICE:

The author has granted a nonexclusive license allowing Library and Archives Canada to reproduce, publish, archive, preserve, conserve, communicate to the public by telecommunication or on the Internet, loan, distribute and sell theses worldwide, for commercial or noncommercial purposes, in microform, paper, electronic and/or any other formats.

The author retains copyright ownership and moral rights in this thesis. Neither the thesis nor substantial extracts from it may be printed or otherwise reproduced without the author's permission.
AVIS:

L'auteur a accordé une licence non exclusive permettant à la Bibliothèque et Archives Canada de reproduire, publier, archiver, sauvegarder, conserver, transmettre au public par télécommunication ou par l'Internet, prêter, distribuer et vendre des thèses partout dans le monde, à des fins commerciales ou autres, sur support microforme, papier, électronique et/ou autres formats.

L'auteur conserve la propriété du droit d'auteur et des droits moraux qui protège cette thèse. $\mathrm{Ni}$ la thèse ni des extraits substantiels de celle-ci ne doivent être imprimés ou autrement reproduits sans son autorisation.
In compliance with the Canadian Privacy Act some supporting forms may have been removed from this thesis.

While these forms may be included in the document page count, their removal does not represent any loss of content from the thesis.
Conformément à la loi canadienne sur la protection de la vie privée, quelques formulaires secondaires ont été enlevés de cette thèse.

Bien que ces formulaires aient inclus dans la pagination, il n'y aura aucun contenu manquant.

\section{Canadä}




\section{Abstract}

In this thesis computational methods were developed to solve three biological problems: to design RNA/DNA pools for in vitro selection of complex aptamers; to identify transposon insertion polymorphisms by computational comparative analysis of next generation personal genome data; and to identify and predict protein complexes from protein-protein interaction networks.

It is well known that using random RNA/DNA sequences as starting pools in in vitro aptamer selection experiments generally yields low complexity structures. Two computational methods were developed to generate sequence pools that exhibit higher structural complexity and can be used to increase the structural diversity of initial pools. Random Filtering increases the number of five-way junctions in RNA/DNA pools, and Genetic Filtering designs RNA/DNA pools with a specified structural distribution. DNA pools designed by these methods were shown to greatly improve access to highly complex sequence structures for aptamer selections.

Structural variations in a genome are a prominent and important type of genetic variation. Among all types of structural variations, the identification of transposon insertion polymorphisms is more challenging due to the highly repetitive nature of transposon sequences. A computational method, TIP-finder, was developed to identify transposon insertions through the analysis of next generation personal genome data. The efficiency of TIP-finder was tested with simulated data and it was able to detect $88 \%$ of transposon insertions with a precision of $\geq 91 \%$. Using TIP-finder to analyze six genomes, a total of 5569 transposon insertions were identified, representing the most comprehensive analysis of such type of genetic variation.

The cluster editing problem is a non-deterministic polynomial-time hard problem. In this thesis, a fixed-parameter tractability based method was implemented and improved for cluster editing. This method was then applied to identify and predict protein complexes of yeast. Results showed that this method has potential to identify known protein complexes and to predict new proteins in the complexes. It also has potential to predict new edges in protein-protein interaction networks as well. 


\section{Acknowledgements}

First and foremost, I would like to thank Professor Frank Dehne for giving me the opportunity to pursue research in his group, to explore the power of computation in challenging biological context, and for his support and patience with my research. My gratitude also goes to my co-superviser, Professor Ping Liang, who provided me with the opportunity, and support, to investigate comparative genomics, a truly fanscinating area with far-reaching significance.

My research, with a multidisciplinary nature, has drawn support from a large number of collaborators, without which I would not have been able to accomplish the projects. My sincerely thank Professors Maria DeRosa, Michael Langston, Ashkan Golshani, James Green, Michel Dumontier, and Yingfu Li for their help and suggestions.

Thanks go to fellow students across various research groups for their expertise and help, Dr. Sylvain Pitre, Dr. Yun Zhang, Maureen McKeague, Irene Lozynska, and Md Alamgir.

Finally I thank my husband Tony for his longtime support and encouragement that made me strong, and my son Alex for all the moments, joyful or otherwise, that kept me going. 


\section{Table of Contents}

$\begin{array}{lll}\text { Abstract } & \text { ii }\end{array}$

$\begin{array}{ll}\text { Acknowledgements } & \text { iii }\end{array}$

List of Figures $\quad$ xi

List of Tables $\quad$ xiii

List of Abbreviations $\quad$ xiv

$\begin{array}{lll}\text { Chapter } 1 & \text { Introduction } & 1\end{array}$

Chapter 2 Computational methods towards the design of pools for in vitro selection of complex aptamers $\quad 7$

2.1 Introduction . . . . . . . . . . . . . . . . . . . 7

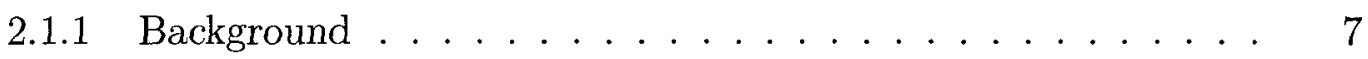

2.1 .2 Statement of the problem $\ldots \ldots \ldots \ldots$

2.1 .3 Contributions . . . . . . . . . . . . . . . . . 10

2.2 Literature review . . . . . . . . . . . . . . . . . . . 12

2.2 .1 What are aptamers? . . . . . . . . . . . . . . 12

2.2 .2 Outline of the SELEX process . . . . . . . . . . . 12

2.2 .3 Applications of aptamers . . . . . . . . . . . . . 13

2.2.4 The secondary structure . . . . . . . . . . . . . . 14

2.2.5 Graphical representation of RNA secondary structures . . . 15

2.2.6 Computational approaches for aptamer pool designs . . . . . 16

2.3 Method description . . . . . . . . . . . . . . . . 18

2.3.1 The Random Filtering method . . . . . . . . . . . . . 18

2.3.2 The Genetic Filtering method . . . . . . . . . . . . . 21

2.3.3 The RNA To Tree method . . . . . . . . . . . . . . 28 
2.4 Results . . . . . . . . . . . . . . . . . . .

2.4.1 Increasing the number of complex structures in SELEX starting pools (Random Filtering method) . . . . . . . . . . . . 30

2.4.2 Designing a diverse SELEX starting pool with uniform structural distribution (Genetic Filtering method) . . . . . . . . 36

2.4.3 Designing a SELEX starting pool with uniform tree structure distribution ....................... 40

2.4.4 Result of SELEX experiment with improved pool design . . . 42

2.5 Discussion of the algorithm approaches . . . . . . . . . . . 44

2.5.1 Choice of secondary structure folding algorithm (ViennaRNA) 44

2.5.2 Comparison of Inverse Folding with Random Filtering . . . . . 47

2.5.3 Comparison of Genetic Filtering with the Mixing Matrices ap-

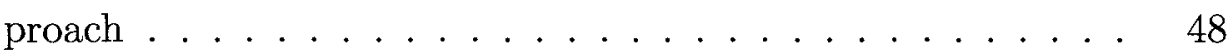

2.6 Conclusions and future work . . . . . . . . . . . . . 49

Chapter 3 Identification of transposon insertion polymorphisms by computational comparative analysis of next generation personal genome data

3.1 Introduction . . . . . . . . . . . . . . . . 51

3.2 Literature review . . . . . . . . . . . . . . 53

3.2 .1 Genome ................... 53

3.2 .2 Genome sequencing . . . . . . . . . . . 54

3.2.3 Genome sequencing strategies ............ 58

3.2.4 The human genome projects ............. 60

3.2.5 Structural variation in the human genome ......... 61

3.2.6 Methods for structural variation detection . . . . . . . . . 62

3.2.7 Transposon insertion polymorphisms . . . . . . . . . . 66

3.2.8 Computational methods for TIPs detection . . . . . . . . . 68

3.3 Methods ......................... 69

3.3.1 General approach . . . . . . . . . . . . . 69 
3.3.2 Identification of TIPs_OUT candidate loci $\ldots \ldots \ldots$

3.3.3 Prediction of the insertion location . . . . . . . . . . 72

3.3.4 Prediction of the insertion size and orientation . . . . . . . 73

3.3.5 Prediction of the insertion transposon subfamily . . . . . . 76

3.3.6 Prediction of the insertion genotype . . . . . . . . . . 77

3.3 .7 TIPs_OUT loci filtering $\ldots \ldots \ldots \ldots \ldots \ldots$

3.4 Results . . . . . . . . . . . . . . . . . . 83

3.4.1 TIPs_OUT identification in the simulated genome . . . . . . 83

3.4.2 TIPs_OUT prediction based on six genomes . . . . . . . . 84

3.4.3 Experimental validation on predicted TIPs_OUT . . . . . . 89

3.5 Discussions . . . . . . . . . . . . . . . . . . . 89

3.6 Conclusions and future work . . . . . . . . . . . . . . . . 91

Chapter 4 The cluster editing problem: implementation, improvement and application $\quad 94$

4.1 Introduction . . . . . . . . . . . . . . . . . . . . . . . . . 94

4.2 State of the art in algorithms for the cluster editing problem . . . . 97

4.2.1 Approximation algorithms . . . . . . . . . . . . 97

4.2.2 Fixed-parameter algorithms for cluster editing . . . . . . . . 99

4.2.3 Review of the algorithms implemented by this study . . . . 101

4.3 Methods . . . . . . . . . . . . . . . . . . . . . . . 107

4.3 .1 A simple greedy heuristic . . . . . . . . . . . . . 107

4.3 .2 An LP approach . . . . . . . . . . . . . . . . . . 108

4.3 .3 An FPT-based approach . . . . . . . . . . . . . . . . 108

4.4 Implementation . . . . . . . . . . . . . . . . . 111

$4.4 .1 \quad$ Experimental setup . . . . . . . . . . . . . . . . . 111

4.4 .2 Experimental results . . . . . . . . . . . . . . . 112

4.5 Improvements . . . . . . . . . . . . . . . . . . . . . . 117

4.5.1 A greedy method for choosing branching orders . . . . . . 117

4.5.2 Dynamically updated matrix for neighbor information . . . . 118 
4.5.3 An LP method for choosing branching orders . . . . . . . 119

4.5.4 A parallel FPT-based method for cluster editing . . . . . . 120

4.5.5 Experimental results after further improvements . . . . . . . 123

4.6 Application . . . . . . . . . . . . . . . . . . . . 127

4.6.1 The cluster editing method to predict protein complexes in $S$. cerevisiae . . . . . . . . . . . . . . . 128

4.6 .2 Experimental results $\ldots \ldots \ldots \ldots$

4.7 Conclusions and future work . . . . . . . . . . . . . . . 134

$\begin{array}{lll}\text { Chapter } 5 & \text { Summary of contributions } & 137\end{array}$

$\begin{array}{ll}\text { Bibliography } & 139\end{array}$ 


\section{List of Figures}

Figure 2.1 Structural distribution of the 2793 known aptamers from the Ellington Database [102]. . . . . . . . . . . . . . . 8

Figure 2.2 The SELEX strategy for the screening of RNA aptamers. . . . 13

Figure 2.3 Elements of RNA secondary Structures. . . . . . . . . . . . . . 14

Figure 2.4 An example of an RNA pseudoknot. . . . . . . . . . . . . . 15

Figure 2.5 Schematic graphical representation for a RNA secondary structure generated by Mfold [191]. . . . . . . . . . . . . . 16

Figure 2.6 Complete sets of tree graphs having two to seven vertices [53]. 17

Figure 2.7 A sample 5-way junction structure used to calculate the method for counting junctions. . . . . . . . . . . . . 20

Figure 2.8 Comparison of junction distribution with different stem lengths. 21

Figure 2.9 Graphical overview of the Genetic Filtering algorithm. . . . . 22

Figure 2.10 Comparison of average structural distribution of 1 million sequences for random RNA/DNA pools and RNA/DNA pools generated by Random Filtering. . . . . . . . . . . . . . . 31

Figure 2.11 Comparison of average structural distribution of 1 million sequences for random RNA/DNA pools and RNA/DNA pools generated by Random Filtering with 15nt primers at both ends, "GGAAGAGATGGCGAC" and "AGCTGATCCTGATGG". . 31

Figure 2.12 Sample secondary structure of RFPool A . . . . . . . . 33

Figure 2.13 DNA structures obtained experimentally from RFPool $A$. . . . 34

Figure 2.14 Sample sequences and representative secondary structures for RFPool A. . . . . . . . . . . . . . . . 35

Figure 2.15 Relationship between 5J population and sequence lengths for a random pool of 1 million sequences with and without primers.

Figure 2.16 The best fitness value observed so far at each generation of Genetic Filtering (primers "ATACCAGCTTATTCAATT" and "AGATAGTAAGTGCAATCT"). . . . . . . . . . . . . 
Figure 2.17 Sample secondary structure of GFPool1. . . . . . . . . . . . 38

Figure 2.18 The best fitness value observed at each generation of Genetic Filtering (primers "ATACCAGCTTATTCAATT" and "AGATTGCACTTACTATCT"). . . . . . . . . . . . 39

Figure 2.19 Sample secondary structure of GFPool2. . . . . . . . . . . 40

Figure 2.20 Sample secondary structure of GFPool3. . . . . . . . . . . . 41

Figure 2.21 Tree structure distribution of GFPool3. . . . . . . . . . . . . 41

Figure 2.22 Percent recovery of DNA eluted from the ATP selection column versus selection round. . . . . . . . . . . . . . 42

Figure 2.23 DNA structures obtained experimentally from the starting GFPooll used in SELEX experiments. . . . . . . . . . . . . 44

Figure 2.24 DNA structures obtained after 8 selection rounds against ATP. $\quad 44$

Figure 2.25 Comparison of predicted structural distributions of RNAfold, GTfold and MCfold for the same 1000 random sequences of 100 nucleotides each (containing no fixed primer sites). . . . . 46

Figure 2.26 Resulting structural distribution after addition of two $15 \mathrm{nt}$ primers to 20 million 5-way junctions sequences of length $70 \mathrm{nt}$ generated by Inverse Folding. . . . . . . . . . . . . . 47

Figure 2.27 Comparison of average structural distribution for the pools generated by Random Filtering and Inverse Folding. . . . . . . . 48

Figure 3.1 Hierachical shotgun sequencing method $[134] . \ldots \ldots$

Figure 3.2 Whole genome shotgun sequencing method. . . . . . . . 59

Figure 3.3 Types of structural variation that can be detected with PEM

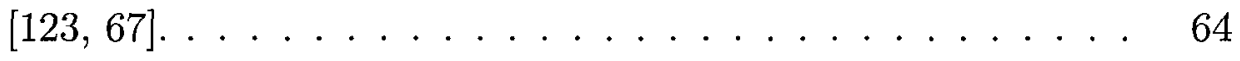

Figure 3.4 The TE content of the human genome. . . . . . . . . 67

Figure 3.5 An example of a TIPs_OUTcandidate locus. . . . . . . . . 71

Figure 3.6 Schematic representation of an insertion split. . . . . . . . . 74

Figure 3.7 Schematic representation of an insertion overlap. . . . . . . . 74

Figure 3.8 The simulated values of cutoffA for different genome coverage. 81 
Figure 3.9 The simulated values of cutoffB for different genome coverage.

Figure 3.10 Relationship between genome coverage and numMF32 cutoff for different genotype. . . . . . . . . . . . . . 82

Figure 3.11 Alu subfamily distribution for 6 genomes. . . . . . . . . . . 86

Figure 3.12 L1 subfamily distribution for 6 genomes. . . . . . . . . . . 86

Figure 3.13 SVA subfamily distribution for 6 genomes. . . . . . . . . . 87

Figure 3.14 HERV subfamily distribution for 6 genomes. . . . . . . . . 87

Figure 4.1 An example of the cluster editing problem. . . . . . . . . . . 95

Figure 4.2 The branching rule for case C2 [57] . . . . . . . . . . 105

Figure 4.3 The branching rule for case C3 [57] . . . . . . . . . . . . . 105

Figure 4.4 A graph $G$ and its critical clique graph $C$ [58]. . . . . . . . 106

Figure 4.5 A comparison of edit distances computed by LP versus the greedy method. . . . . . . . . . . . . . . . . . 112

Figure 4.6 A comparison of run times required by LP versus the greedy method. . . . . . . . . . . . . . . . . . . 112

Figure 4.7 FPT run times on a graph with 50 vertices, 5 clusters and edit distance $20 \ldots \ldots \ldots \ldots \ldots$. . . . . . . . . . 113

Figure 4.8 FPT run times on a graph with 100 vertices, 10 clusters and edit distance 40. . . . . . . . . . . . . . . . 114

Figure 4.9 The monotonicity of FPT parameter effects as seen when solving clique with vertex cover. . . . . . . . . . . . . . . . 114

Figure 4.10 FPT parameter effects on a graph with 100 vertices, 5 clusters and edit distance $40 \ldots \ldots \ldots \ldots \ldots$

Figure 4.11 FPT parameter effects on a graph with 100 vertices, 10 clusters and edit distance $40 \ldots \ldots \ldots \ldots \ldots \ldots$

Figure 4.12 Search trees depend on parameter values. . . . . . . . . . . 116

Figure 4.13 The scalability of approximation via LP versus optimization via FPT . . . . . . . . . . . . . . 117 
Figure 4.14 A comparison of run time before and after the FPT improvement methods. . . . . . . . . . . . . . . . . . . . 124

Figure 4.15 A comparison of edit distances computed for Yes case before and after the FPT improvement methods. . . . . . . . . . 124

Figure 4.16 FPT run times on a graph with 20 vertices and 95 edges. . . . 125

Figure 4.17 A comparison of run time on graph 100v_10c_40ed_491e between the LP method and the greedy method for choosing branching orders. . . . . . . . . . . . . . . . 125

Figure 4.18 A comparison of run time on graph 20v_95e among the LP method, the greedy method and the parallel method for branching. . . . . . . . . . . . . . . . . 126

Figure 4.19 The run time on the graph $81 v \_269 e$ by the parallel FPT-based method. . . . . . . . . . . . . . . . . . 


\section{List of Tables}

Table 2.1 Structural distribution determined with different numbers of mutations (averages and variances in brackets). . . . . . . . .

Table 2.2 Structural distribution of sequences created by mutating 50 different 3J sequences 1 million times each. . . . . . . . . . 23

Table 2.3 in silıco and in vitro structural distribution for RFPool $A$. . . . 32

Table 2.4 Structural distribution for GFPool1. . . . . . . . . . . . . . . 37

Table 2.5 Structural distribution, distance from the optimal, and number of single stranded positions present for GFPool2 and GFPool2A. 39

Table 2.6 in sılıco structural distribution, distance from the optimal, and number of single stranded positions present for DNA pools with primers as well as experimental structural distributions prior to starting SELEX and after 8 rounds of selection using ATP as the target. . . . . . . . . . . . . . .

Table 2.7 Structural distribution comparison between RNAfold and GTfold for GFPool1. . . . . . . . . . . . . . . . . . 46

Table 3.1 Summary of the capacity for the major next-generation sequencing technologies [175]. . . . . . . . . . . . 57

Table 3.2 List of the time complexity of the TIPs_OUTclusterFinding al-

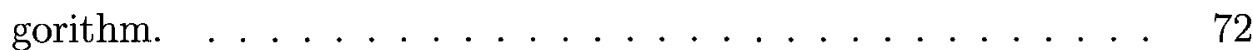

Table 3.3 Summary of filtering parameters used in TIP-finder. . . . . . . 82

Table 3.4 Summary of TIPs_OUT predictions based on simulation. . . . . 84

Table 3.5 Sample results of TIPs_OUT prediction. . . . . . . . . . 85

Table 3.6 Summary of TIPs_OUT predictions based on the six genomes. . 85

Table 3.7 Summary of experimental validation on predicted TIPs_OUT. . 89

Table 4.1 The approximation results on the problem of minimizing dis-

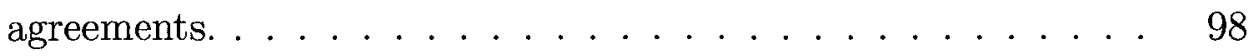

xii 
Table 4.2 The branching order for case $C 2$ of the refined branching strategy based on the LP method for choosing branching order. . . . . . 120

Table 4.3 The branching order for case $C 3$ of the refined branching strategy based on the LP method for choosing branching order. . . . . . 121

Table 4.4 Comparison of protein clusters found by cluster editing with known protein complexes. . . . . . . . . . . . . . . . . . 131

Table 4.5 Comparison of predicted edges with newly updated protein-protein interaction database. . . . . . . . . . . . . . . . 133

Table 4.6 Summary of the implementation of cluster editing method with overlapping on 6 test graphs. . . . . . . . . . . . . . . 134

Table 4.7 Protein clusters predicted for Test Graph 2 after the first and the second round cluster editing. . . . . . . . . . . . . . 134

Table 4.8 Summary of the graphs generated from a list of proteins that are involved in non-homologous end-joining process. . . . . . 135

Table 4.9 Summary of the edit distances found by cluster editing for the protein graphs that are involved in non-homologous end-joining process. . . . . . . . . . . . . . . . . . 


\section{List of Abbreviations}

DFS Depth-First Search

FPT Fixed-Parameter Tractability

HGP Human Genome Project

HPC High Performance Computing

LP Linear Programming

NGS Next-Generation Sequencing

NP Non-deterministic Polynomial-time

PCR Polymerase Chain Reaction

PEM Paired-End Mapping

PPI Protein-Protein Interaction

SELEX Systematic Evolution of Ligands by Exponential Enrichment

SNP Single-Nucleotides Polymorphism

SRP Signal Recognition Particle

SS Single-Stranded

SV Structural Variation

TE Transposable Element

TIP Transposon Insertion Polymorphism

WGS Whole Genome Shotgun 


\section{Chapter 1}

\section{Introduction}

Bioinformatics represents a field integrating molecular biological science and information science such as applied mathematics, system science, computer science, and statistics to understand and organize the information associated with biomolecule, on a large scale [112]. It focuses on developing and applying computational techniques to increase the understanding of biological processes. Bioinformatics uses computer programs to reveal fundamental mechanisms underlying biological problems related to the structure and function of macromolecules, biochemical pathways, disease processes, and evolution [134].

According to a National Institutes of Health definition [128], bioinformatics is "research, development, or application of computational tools and approaches for expanding the use of biological, medical, behavioral or health data, including those to acquire, store, organize, analyze, or visualize such data." The related discipline of computational biology is "the development and application of data-analytical and theoretical methods, mathematical modeling and computational simulation techniques to the study of biological, behavioral, and social systems." It has been very well documented that bioinformatics has become an indispensable tool in advancing our knowledge of biological phenomena and fostering new biomedical applications.

During the last few decades, due to the vast amount of biological data generated particularly in the fields of genomics and proteomics, bioinformatics research has become increasingly demanding on the computational capacity. It requires faster CPU, larger memory and faster I/O when analyzing these biological data. With the increase of such requirements on computational tools, high performance computing (HPC) has become a critical component in bioinformatics. HPC uses supercomputers and computer clusters to solve advanced computation problems. Through parallel processing algorithms and software, computational problems can be divided into small 
pieces so that each can be executed simultaneously by separate processors. HPC provides more efficient computation and memory usage, and when it is applied to the field of bioinformatics, it allows researchers to explore complex biological problems more efficiently.

In this thesis computational methods were developed to solve three different biological problems: to design pools for in vitro selection of complex aptamers; to identify transposon insertion polymorphisms (TIPs) by computational comparative analysis of next generation personal genome data; and to identify and predict protein complexes from protein-protein interaction networks. HPC was indispensable in all three projects to provide the computational power that is essential to handle large-scale data involved.

Aptamers are single stranded nucleic acids that bind to target molecules with high affinity through 3D conformation complementarity. They are useful in biotechnological and therapeutic applications $[48,164,17,77]$. Aptamers are usually generated by an in vitro selection process known as SELEX (Systematic Evolution of Ligands by Exponential Enrichment) from a large random sequence pool.

It is well known that using random RNA/DNA sequences for SELEX experiments will generally yield low complexity structures such as 1-way and 2-way junction sequences, and that the highly complex structures such as 4-way and 5-way junction sequences are far less abundant [84, 190, 99]. For example, aptamers that bind to ATP, chloramphenicol, neomycin B and streptomycin all have linear stem-loop or slightly branched structures [99]. In a recent computational analysis of in vitro RNA random pools [53], it was shown that random pools are not structurally diverse. They heavily favor simple topological structures due to incomplete and insufficient random sequence sampling. This lack of structural diversity in random pools suggests that complex structure motifs with high-order junctions would be rare in selected aptamers.

Recent experimental results suggest that structurally diverse libraries which, for instance, include high-order junctions, may prove useful in finding new functional motifs $[24,23]$. In light of these findings, it would become advantageous to engineer the starting pool to increase highly complex structures and to have uniform distribution 
over all possible structures. Although a number of approaches have been used to increase the structural complexity of starting pools [78, 130, 131, 187, 100, 94, 104, 36], these approaches are based on ad hoc pool designs for individual experiments and targets. Hence, it is necessary to develop more general pool design approaches that can be used for any targets.

In this thesis two computational methods were developed to generate sequence pools that exhibit higher structural complexity and that can be used to increase the overall structural diversity of initial pools for in vitro selection experiments. Random Filtering selectively increases the number of five-way junctions in RNA/DNA pools, and Genetıc Filtering designs RNA/DNA pools to a specified structure distribution, whether uniform or otherwise. We showed that using our computationally designed DNA pools greatly improved access to highly complex sequence structures for SELEX experiments without losing the ability to select for common 1-way and 2-way junction sequences. Part of this work has been published in RNA [111].

Structural variations (SVs) in genomes are now known as a prominent and important type of genetic variation. They can be defined as genetic alternation comprising microscopic and submicroscopic variants including deletion, duplication, copy-number polymorphism, insertion, inversion and translocations [50]. Rapidly accumulating evidence indicates that SVs are likely to make an important contribution to human diversity and disease susceptibility $[66,115,120]$. However, identification of such SVs has proven to be a very challenging task [41]. In recent years, with the development of new sequencing technologies such as 454 sequencing, Solexa technology, and the SOLiD platform, vast amount of next-generation sequencing (NGS) data has been generated. These technological advancements opened up new opportunities for the identification of critical SVs associated with phenotypes from these NGS data. The availability of rapidly increasing personal genome sequence data from the use of newer generations of sequencing technologies provides a very rich data source for surveying SVs in humans. Nevertheless, processing the personal genome data in a form of billions of short sequence reads for the identification of SVs represents a very challenging computational task.

Transposon insertion polymorphism (TIP) is one type of SVs. Transposons are 
discrete pieces of DNA that can move within a genome, and they account for approximately $45 \%$ of the human genome [34]. By simply moving from one place to another or by making new copies into other locations in the genome, transposons play important roles in the evolution of the genome and human genetic diversity. Today, a small fraction of transposons remain active in the human genome including subfamilies of Alu, L1, SVA, and possibly HERV-K elements [126]. Transposon insertion polymorphism refers to the presence or absence of a transposon insertion at a specific genomic location in populations of a given species. A polymorphism refers to a common variation in the DNA that has a frequency of at least $1 \%$ in the population, whereas a variation which is rare or uncommon in the population is called a private polymorphism [13]. In this study, all types of transposon insertion variations are referred to as transposon insertion polymorphisms.

Since an extremely large number of copies of transposons are present in the human genome and their sequences among members in the same family are very similar, the identification of TIPs is more challenging than others SVs. To this point, only a small number of TIPs have been identified using classical molecular biology techniques, such as locus-specific PCR, targeted mutation screening, and transposon differential display polymerase chain reactions (PCR) $[185,5,11,146]$. With the availability of human genome sequences generated from the human genome project and personal genome project, a few studies have used computational comparative genomic approach to identify TIPs, but most methods are limited to compare two assembled genome sequences. However, until now only few human genome sequences have been assembled, i.e. the human genome reference sequences and Venter's personal genomes. Most human genome sequences generated by next-generation sequencing technologies are unassembled. Therefore, it is necessary to develop a computational method to identify TIPs by computational comparative analysis of next generation personal genome data, that is, to compare a sequenced but unassembled genome (test genome) with an assembled genome (reference genome).

In this thesis, a computational method, TIP-finder, was developed to identify TIPs through the analysis of next generation personal genome data. TIP-finder predicts TIPs via a greedy algorithm based on the mapping patterns of pair-end reads 
associated with TIPs and other biological characteristics of transposons. We tested the efficiency of TIP-finder using a simulated genome containing a set of known TIPs and were able to detect about $88 \%$ of TIPs with a precision of $\geq 91 \%$. Using TIPfinder to analyze the Solexa pair-end sequence data at deep coverage for six genomes representing two trio families generated by the 1000 genome project, we identified a total of 5569 TIPs, consisting of 4881, 456, 91, and 141 insertions from Alu, L1, SVA and HERV, respectively, that are absent in the human reference genomes. In addition to the estimated insertion location, TIP-finder also provides prediction of transposon subfamily, insertion size and genotype for each TIP. This study demonstrates the high efficiency of TIP-finder for the identification of TIPs, and it represents one of the few comprehensive analysis of TIPs performed so far and reveals an unexpectedly highly level of transposon associated genetic polymorphsim in humans. Part of this work has been submitted to the American Institute of Physics Proceedings of AMMCS-2011 [110] and a full paper is under preparation to be submitted for publication.

The cluster editing problem, which is a non-deterministic polynomial-time hard (NP-hard) problem, was also studied in this thesis. The cluster editing problem is defined as follows. Given an undirected graph $G=(V, E)$, and a non-negative integer $k$, is it possible to transform $G$ through at most $k$ insertion and deletion into a cluster graph? A cluster graph is a graph that consists of a disjoint union of cliques. A clique is a graph where every two vertices have an edge between them. We present the first practical implementation of a fixed-parameter tractability (FPT)-based method for cluster editing, and compare our implementation with the straightforward greedy method and a solution based on linear programming (LP). Our experiments showed that the best performance was obtained by using the refined branching method together with interleaving (re-kernelization). We then improved the FPT-based method by using a number of algorithms for the cluster editing problem.

A protein complex refers to a group of two or more proteins with a similar function that highly physically interact with each other. It corresponds to a dense subgraph in the protein-protein interaction (PPI) networks [7, 141]. Protein complexes carry out many important molecular functions. Therefore, identification of protein complexes can help to understand and explain certain biological processes. We applied the 
improved cluster editing method to identify and predict protein complexes of yeast. Comparison of protein clusters found by the cluster editing method with known yeast protein complexes showed that this method has potential to identify known protein complexes and to predict new proteins in the complexes. It also has potential to predict new edges in PPI networks. Part of this work has been published in Springer LNCS 4169 [37].

The thesis consists of five chapters. Chapter 2 first introduces the background about aptamer pool design. It then reviews current experimental as well as computational methods for designing aptamer pools. Next, two methods to design the pools are described: Random Filtering and Genetic Filtering. The results of these two methods and experimental validation are explained and discussed. Chapter 3 first provides background about genome sequencing, human genome projects, SVs and TIPs. It then reviews the current computational methods for the identification of SVs and TIPs. Next, it describes the TIP-finder method to identify TIPs, followed by the results of applying this method to a simulated genome and 6 genomes from the 1000 genome project. The experimental validation is also explained and discussed. Chapter 4 first introduces the cluster editing problem and reviews the state of the art in algorithms for this problem. It then presents our implementation and improvement of a FPT-based method for cluster editing. The applications of the FPT-based method for cluster editing to predict protein complexes is discussed next. Finally, Chapter 5 summarizes the contributions of this study. 


\section{Chapter 2}

\section{Computational methods towards the design of pools for in vitro selection of complex aptamers}

This chapter describes the development and implementation of computational methods towards the design of pools for in vitro selection of complex aptamers. A paper published by [111] is partly the basis for this chapter. I carried out all computational work for this project. The SELEX experiments were performed by Maureen McKeague in Dr. DeRosa's laboratory at Carleton University.

\section{$2.1 \quad$ Introduction}

\subsubsection{Background}

Aptamers are target-binding nucleic acid molecules that can bind with high specificity to a range of molecules such as antibiotics, proteins, viruses, catalytic RNA molecules and even whole cells such as live cancer cells $[181,61,63,157]$. Methods for the generation of aptamers were first established by Gold and Szostak [44, 171]. To date, over 3,400 aptamers that bind to a wide variety of ligands have been selected by a biochemical process known as Systematic Evolution of Ligands by Exponential Enrichment (SELEX) [102, 170]. SELEX involves the repetitive partitioning of a library of random nucleic acid sequences on the basis of selective binding to the desired target, followed by amplification by polymerase chain reaction.

Recent in vitro selection experiments have led to the identification of novel aptamers for biotechnological purposes such as functional genomics [48] and biosensing [164]. Other recent applications of aptamers include control of gene expression and medical diagnosis $[17,77]$. As the applications of aptamers grow, the need for design, identification and selection of novel and improved aptamers becomes even more apparent. 


\subsubsection{Statement of the problem}

It is well known that in vitro random RNA/DNA pools are not structurally diverse and heavily favour simple topological structures such as stem-loops structures due to incomplete and insufficient random sequence sampling [53]. Analysis of existing aptamers has revealed that the majority of oligonucleotide sequences generated from random in vitro selection experiments have simple structures with low degrees of complexity and that highly complex structures are far less abundant [84, 190, 99]. For example, aptamers that bind to ATP, chloramphenicol, neomycin B and streptomycin all have linear stem-loop or slightly branched structures [99]. This lack of structural diversity in random pools may explain why complex structure motifs such as highorder junctions (see the definition of junction in Section 2.2.4) are rare in known synthetic aptamers.

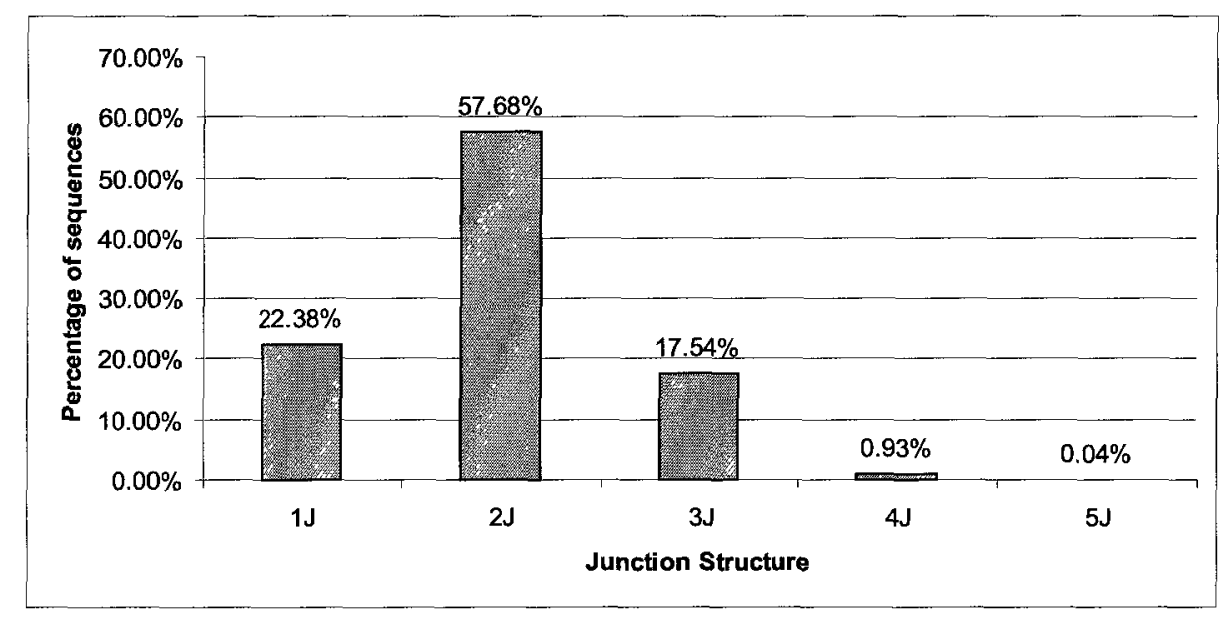

Figure 2.1: Structural distribution of the 2793 known aptamers from the Ellington Database [102].

To further demonstrate this, we folded the 2793 known aptamers from the Ellington Lab aptamer database [102] by ViennaRNA [64], and then counted the number of junctions of these known aptamers using the CountJunctions algorithm described in Section 2.3. The results showed that among these known aptamers, less than $1 \%$ of them contain 4-way and 5-way junction structures (see Figure 2.1). However, natural aptamers (i.e. riboswitches) more commonly contain high-order junctions. For example, the lysine riboswitch involves three-helical and two-helical bundles joined 
by a 5-way junction [156]. Almost all other riboswitches can be classified into one of two types based on their high-junction number. Type I riboswitches contain a fundamental 3-way junction architecture. In Type II riboswitches, such as the SAM-I binding riboswitch, the aptamer domain is arranged in a 4-way junction structure [152].

It has recently been shown that increasing the structural diversity of the starting oligonucleotide pool can enhance the possibility of finding novel aptamers with improved activity $[24,23]$. In a recent investigation, five-way junctions (5Js) were found to provide the scaffold for the formation of functionally more active catalytic deoxyribozymes [29]. It was shown that the structures with 5Js were evolved from pre-existing and less complex structures with three-way junctions, and that the more complex structures had a significantly higher rate of activity.

Consequently, novel approaches have been employed to increase the structural complexity of starting pools. It has been shown that structural complexity is largely dependent on sequence length [147]. However, RNA/DNA pools for SELEX are usually restricted to sequences of at most 100 nucleotides (100nt) in length due to the difficulty involved in the synthesis above this length. This 100nt starting sequence typically includes 15 -nucleotide primers at both the 3' and 5' ends, which reduces the total number of variable positions from 100 to 70 . Thus, effective methods should make use of primers as part of their strategy for predicting high complexity structures.

One approach to increase the structural complexity is to keep the structure constant and introduce random segments in the proximity of the existing structure such as group I ribozyme domain $[78,130,131,187]$, and purine nucleotide synthase ribozymes [100]. Other approaches involve changing sequence length and composition [94, 104]. Davis and Szostak [36] isolated a high-affinity GTP aptamer from a partially structured RNA library, one half of which consisted of fully random sequences and the other half of a random region with a designed stem-loop in the middle. Subsequent analysis of the obtained aptamers revealed that most of those with highest affinity originated from the partially structured portion of their pool. They also observed that the highest affinity aptamers had fairly complex structures [24] and concluded from their experiments that more complex RNA structures are required for greater 
GTP-binding activity.

However, the above observations are based on ad-hoc pool designs for individual experiments and targets. Furthermore, these ad-hoc pools with more complex structures were generated by inserting particular stem-loops that were known a priori to be relevant for the given target [36]. Such prior knowledge is not available in many cases. Hence, it is necessary to develop more general pool design approaches that can be used for targets without such prior knowledge. Recently, Schlick and coworkers $[86,88,87]$ developed a computational approach for designing structured RNA pools by modeling the pool generation process using mixing matrices. However, the scope of this approach was limited and the approach is not computationally efficient (see Section 2.5.3).

\subsubsection{Contributions}

We have studied the design of RNA/DNA pools for in vitro selection experiments and developed two new methods to improve their structural complexity and diversity: 1) a computational method called Random Filtering to selectively increase the number of highly complex structures, including five-way junctions in RNA/DNA pools, and 2) a computational method called Genetic Filtering to design RNA/DNA pools with any given structure distribution, including a uniform structure distribution (i.e. 20 percent 1-way (1J), 2-way (2J), 3-way (3J), 4-way (4J) and 5-way (5J) junctions each).

Our first aim was to increase the structural complexity of RNA/DNA pools. Since RNA/DNA secondary structures can be described using unlabeled tree graphs [52], we used the degree of branching, i.e. the number of junctions, to measure their structural complexity. In this approach, a tree graph with a higher order junction has a greater structural complexity. Random Filtering led to a significant enrichment in the number of highly complex structures in DNA pools. For example, pools were generated where more than $10 \%$ of the sequences are five-way junctions and more than $15 \%$ of the sequences are four-way junctions as compared to nearly zero fiveway junctions and around $0.2 \%$ four-way junctions in a typical random DNA pool. We found very similar results for 100nt DNA pools where 30nt are fixed primer-binding 
regions.

Our second aim was to engineer a DNA/RNA pool with any given structure distribution over all possible structural complexities, to maximize the diversity of DNA/RNA sequences available in the starting pool for in vitro selection. For a given set of primers determined by the respective SELEX experiment, our Genetic Filtering method can provide a roadmap for creating a RNA/DNA pool with nearly uniform structure distribution (i.e. $20 \%$ of each 1-way, 2-way, 3-way, 4-way and 5-way junctions). We also extended our Genetic Filtering method to design structured RNA and DNA pools with desired tree structure distributions. Gan and coworkers [52] developed and applied graphical representation and enumeration of 2D RNA topologies to systematically catalog libraries of existing and hypothetical motifs. In their approach, an RNA structure is represented by a tree graph where stems correspond to edges and loops/bulges/junctions to vertices. Based on their tree structure catalog libraries, we used our Genetic Filtering method to generate RNA and DNA pools with a desired motif distribution.

We tested the hypothesis that our designed pools can increase the chance of sampling more complex oligonucleotide structures during in vitro selection. It has already been observed by Davis and Szostak [36], for the case of GTP aptamers, that pools based on customized starting sequences may increase the likelihood of finding better aptamer sequences. To further test this hypothesis, we used a DNA sequence designed with our Genetic Filtering method to generate a uniform structure distribution for the starting pool of a SELEX experiment to select for ATP-binding DNA aptamers. We found that after 8 rounds of selection cycles, complex structures such as five-way junctions accounted for $20 \%$ of the sampled sequences. These experiments confirmed that our methods greatly improved the likelihood of generating sequences of increased structural complexity for SELEX experiments. 


\subsection{Literature review}

\subsubsection{What are aptamers?}

Aptamers are single stranded RNA or DNA molecules (oligonucleotides) that bind specifically with their targets with dissociation constants in the picomolar to nanomolar range. Aptamers are relatively small nucleic acid molecules, with molecular weight around 8-14 kDa. Many aptamers have complex three-dimensional structures, produced by a combination of Watson-Crick and non-canonical intramolecular interactions, and other non-covalent interactions such as electrostatic, van der Waals force, and $\pi$ stacking. Although both DNA and RNA aptamers can form complex secondary and tertiary structure, the range of three-dimensional structures achieved by RNA aptamers are more diverse than DNA aptamer, and also RNA secondary structures are more stable than DNA due to stronger stacking energies [91]. Generation/evolution of aptamers is typically performed by an in vitro process known as SELEX [44, 171].

\subsubsection{Outline of the SELEX process}

As shown in Figure 2.2, in the selection of RNA aptamer via the SELEX process, a large library of randomized DNA sequences, usually in the $10^{14}-10^{15}$ range, is first generated, for example, by solid phase oligonucleotide synthesis. This pool of DNA sequences is then transcribed into an RNA pool using polymerase promoted reactions. In the next step, these RNA molecules are subjected to a selection process. Typically, a mixture of these RNA molecules is passed through a column containing a matrix functionalized with the desired ligand. When eluted with appropriate buffer solutions, RNA molecules that do not bind the ligand are eluted off the column first, leaving those that do bind the ligand. By changing the eluting conditions, RNA molecules that bind the ligand can be recovered from the column matrix. These latter RNA molecules (enriched RNA pool) are then reverse-transcribed into DNAs by reverse transcriptases and then amplified by polymerase chain reactions (PCR) to complete one cycle/round of selection. This selection cycle is typically repeated 8-12 rounds until RNA aptamers with desirable binding affinity are evolved. The identities of these aptamers are then determined by sequencing. In contrast, when DNA aptamers are 
selected, the general methodology is the same, except that i) DNA pools are used directly for selection, and ii) consequently no transcription and reverse-transcription are required.

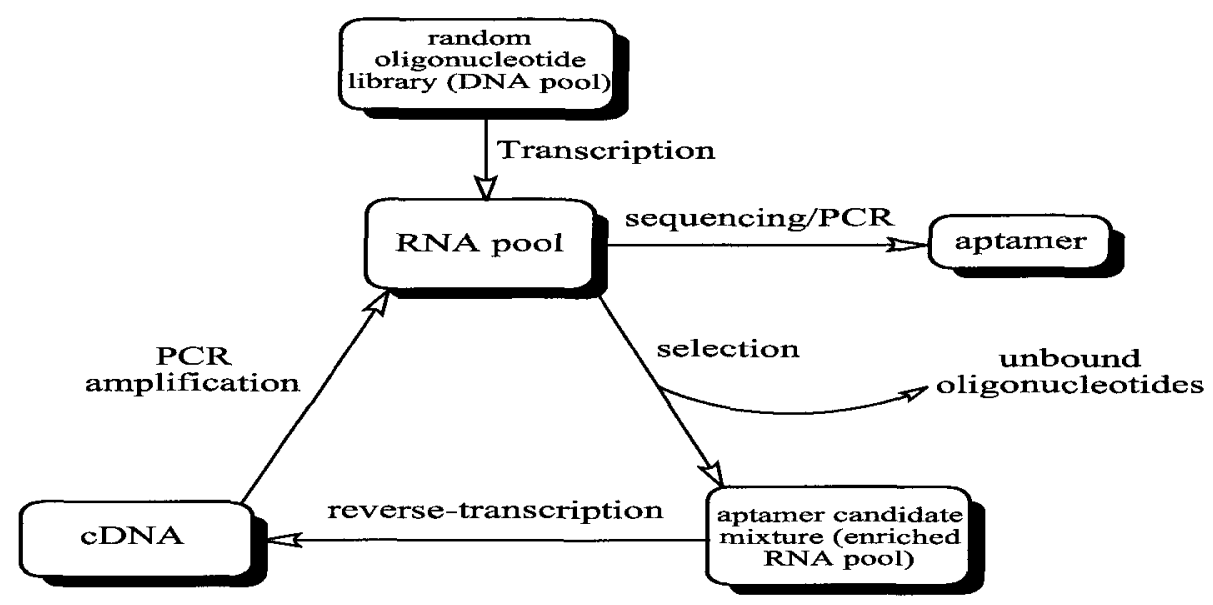

Figure 2.2: The SELEX strategy for the screening of RNA aptamers.

\subsubsection{Applications of aptamers}

Using SELEX, over 3,400 aptamer sequences have been generated in the past two decades $[102,170]$. These aptamers show very high binding affinity with a variety of ligands, including small molecules (such as carbohydrates, antibiotics, and nucleosides) [92], proteins [93], and most recently whole cells [157]. In a number of aspects, aptamers compare favorably with antibodies [83]. First, their binding affinity is comparable. Second, aptamers are generated in vitro, and therefore no animals are involved. Third, the target for aptamer creation can be any molecule, including toxins. Also, once aptamers are successfully selected, they can be chemically synthesized and purified in a reproducible manner. Therefore, aptamers have been under investigation for their potentials in a wide range of applications such as biosensor, disease diagnosis, pathogen detection, and disease treatment. The first aptamer drug (Macugen) has recently been approved by the US Food and Drug Administration to treat age-related macular degeneration. 


\subsubsection{The secondary structure}

Nucleic acids, most notably single-stranded RNA molecules, display complex secondary structures. Some common secondary structures are shown in Figure 2.3. RNA secondary structure is generally divided into helices and various kinds of loops. Helices are defined as contiguous base pairs and loops are defined as unpaired nucleotides surrounded by base pairs. The stem-loop structure, which is also called hairpin loop, is extremely common structural component of nucleic acids. In the stem-loop structure, a base-paired helix ends in a short unpaired loop. Internal loops and bulges are also frequently present in nucleic acids. Internal loops are a short series of unpaired bases in a longer paired helix and bulges are the regions in which one strand of a helix has unpaired bases. Pseudoknots (see Figure 2.4) contain at least two stem-loop structures in which two stems are not disjoint, but one stem is not contained in the other either.

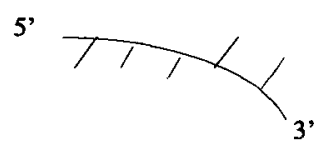

Single strand

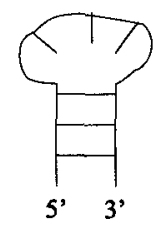

Hairpin loop

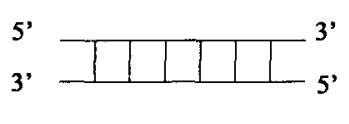

Double helix

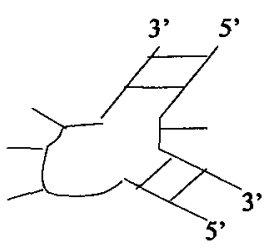

Internal loop

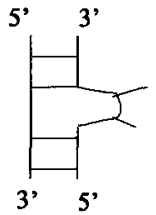

Bulge

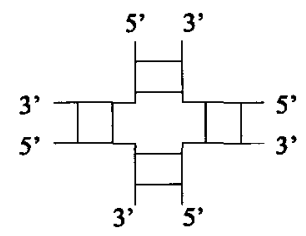

Four-stem junction

Figure 2.3: Elements of RNA secondary Structures.

RNA junctions are defined as the point of connection between different helical segments [108]. They are common secondary-structure elements which present in a wide range of RNA molecules. RNA junctions are important for the structural and catalytic properties of RNAs. It has been shown that they are involved in a 


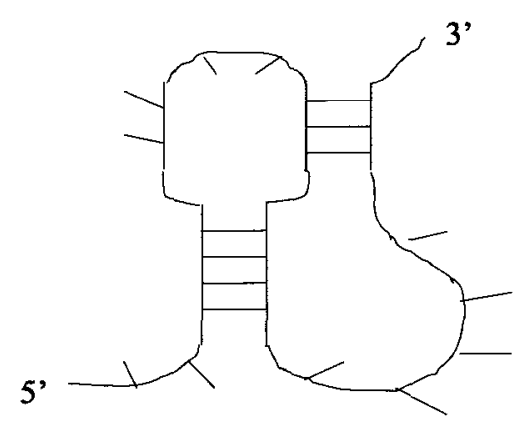

Figure 2.4: An example of an RNA pseudoknot.

variety of different functional roles in nucleic acid, including the self-cleaving catalytic properties of the hammerhead ribozyme [153], promotion of functional folded states of the hairpin ribozyme [182], recognition of the binding pocket domain by purine riboswitches [10, 156], and translation initiation of the hepatitis $\mathrm{C}$ virus at the internal ribosome entry site [85].

\subsubsection{Graphical representation of RNA secondary structures}

Since RNA secondary structures are essentially 2D networks, they can be represented using graphs to facilitate analysis of RNA structures. Such ideas have been applied to analyze structural similarity between RNAs by Le et al. [101] and Shapiro et al. [158]. In 2003, Gan et al. [52] developed a two-dimensional graphical representation approach for describing and estimating the size of RNA's secondary structural repertoire, including naturally occurring and other possible RNA motifs.

To represent a secondary structure as an unlabeled tree graph, Gan et al. [52] used the following rules to assign edges and vertices:

(1) A bulge, hairpin loop, or internal loop is considered as a vertex when there is more than one unmatched nucleotide or noncomplementary base pair.

(2) A junction is considered as a vertex.

(3) The 3'- and 5'-ends of a helical stem are considered as a vertex. 
(4) An RNA stem, defined as having more than one consecutive complementary base pair, is represented as an edge.

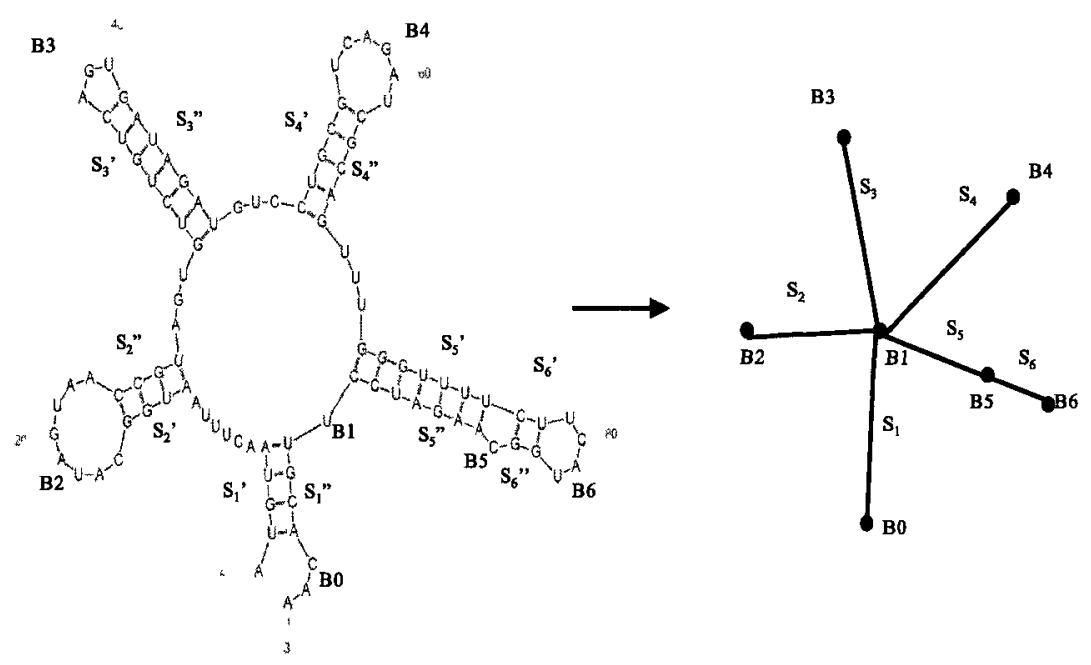

Figure 2.5: Schematic graphical representation for a RNA secondary structure generated by Mfold [191].

As shown in Figure 2.5 the above rules reduce the 2D structures of a RNA to a tree graph. Note that Gan et al. define an RNA stem to have two or more complementary base pairs. Based on this approach, Gevertz et al. [53] summarized the complete sets of tree graphs having two to seven vertices (see Figure 2.6). Each graph is assigned an identification number (ID). This ID is indexed as $n_{m}$, where $n$ is the motif's vertex number $(V)$, and $m$ indicates the order in which the motif occurs within the $V$-vetex tree structures. For example, motif number $5_{1}$ and $7_{3}$ refer to specific five- and sevenvertex trees, respectively. Note that the RNA secondary structures in all figures in this Chapter were generated by Mfold[191].

\subsubsection{Computational approaches for aptamer pool designs}

As discussed in Section 2.1.2, recent findings show that increased structural diversity of the starting oligonucleotide pool can enhance the possibility of finding novel aptamers with improved activity $[24,23]$. However, random pools are not structurally 

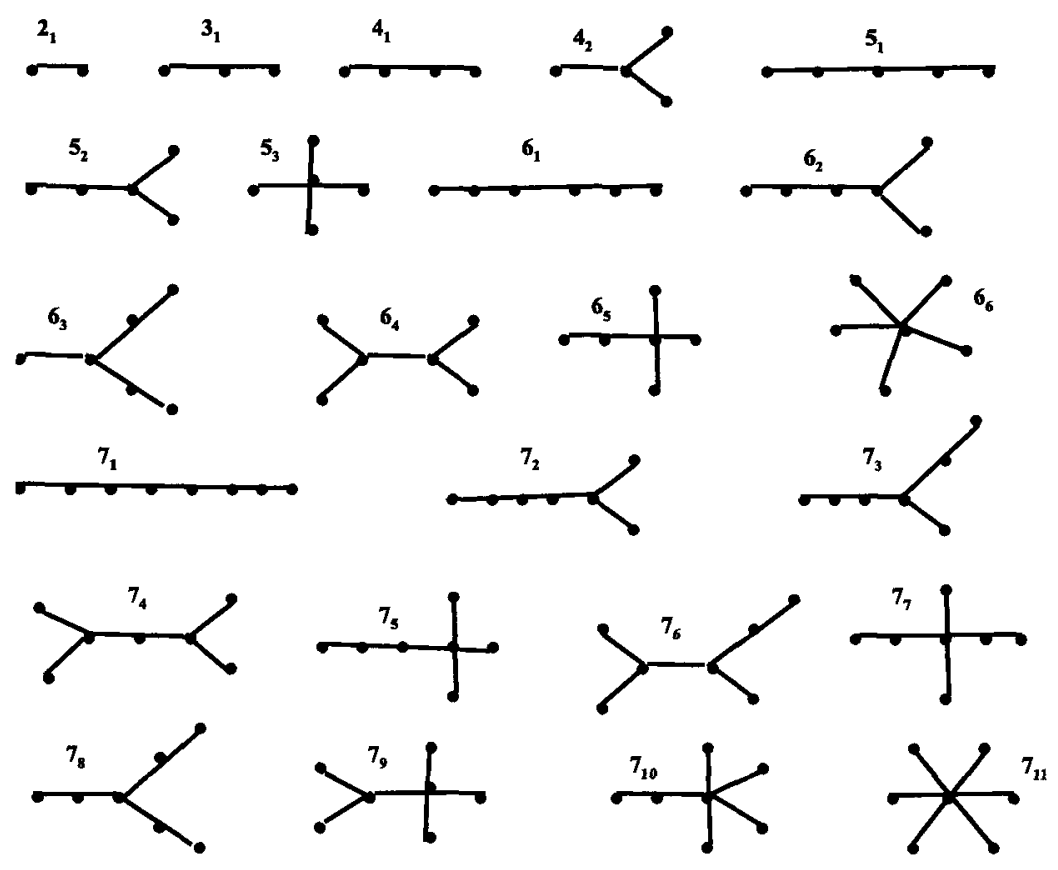

Figure 2.6: Complete sets of tree graphs having two to seven vertices [53].

diverse and heavily favor simple structures such as hairpin loops [53]. Therefore, a number of approaches have been used to increase the structural complexity of starting pools. Nevertheless, these approaches are based on ad hoc pool designs for individual experiments and targets. Hence, it is necessary to develop more general pool design approaches that can be used for any targets.

Schlick and coworkers developed a computational approach for designing structured RNA pools by modeling pool generation process using mixing matrices and analysis of pool structural distribution using tree graphs [86]. The mixing matrix is a 4 by 4 matrix which specifies nucleotide transition rates for all nucleotide bases. Pool synthesis uses four vials (A, C, G, U), and the matrix elements represent nucleotide base composition in a vial. For example, $M_{A C}$ is the molar fraction of base $\mathrm{C}$ to base A in the vial. Schlick and coworkers defined five classes of 22 mixing matrices based on sequence transformations associated with covariance mutations. The mixing matrices are applied to chosen sequences from 6 starting sequences to yield designed sequence pools. Schlick and coworkers also developed a companion web server, RAGPOOLS, for designing and analyzing structured pools for in vitro selection [88]. RAGPOOLS 
provides optimized starting sequences, mixing matrices, and associated weights in response to a user-specified target pool structure distribution.

However, the scope of this approach was limited and the approach is not computationally efficient, for example, the most complex structures created via the mixing matrix approach are $4 \mathrm{~J}$ structures; the error rate generated to create a pool with a uniform distribution of 12 tree structures (from $2_{1}$ to $6_{5}$ defined in [53]) is $60 \%$ using 1 mixing matrix, which is high. A detailed discussion is shown in the Section 2.5.3. Therefore, it is necessary to develop a more accurate approach to design structured RNA/DNA pools.

\subsection{Method description}

We have developed two new methods to increase the structural complexity and diversity of RNA/DNA pools: 1) a computational method called Random Filtering to selectively increase the number of five-way junctions in RNA/DNA pools, and 2) a computational method called Genetic Filtering to design RNA/DNA pools with any given structure distribution, including a uniform structure distribution (i.e. 20 percent 1-way (1J), 2-way (2J), 3-way (3J), 4-way (4J) and 5-way (5J) junctions each).

We also extended our Genetic Filtering method to design structured RNA and DNA pools with desired tree structure distributions. As discussed in Section 2.2.5, an RNA structure can be represented by a tree graph where stems correspond to edges and loops/bulges/junctions to vertices. We have developed a method called $R N A$ Filtering to convert RNA structures to tree graphs so that we can use our Genetic Filtering method to design RNA and DNA pools with a desired motif distribution.

\subsubsection{The Random Filtering method}

Random Filtering is a computational method to selectively increase the number of five-way junctions in DNA pools for SELEX. Random Filtering proceeds as follows: We start from a random RNA or DNA pool and use ViennaRNA (see discussion below) to generate their secondary structures. We then compute the number of junctions for each sequence with the CountJunctions algorithm outlined below. Each $5 \mathrm{~J}$ sequence is then mutated at every single stranded position by randomly choosing 
one of four bases (ACGT) to substitute for the original. This process is repeated 1 million times for each 5J sequence to calculate the structure distribution of each respective pool design. Sequences are only mutated at non-primer positions. The pool with the largest percentage of 5-way junctions is selected.

It was initially unclear whether using a million rounds of mutations was sufficient to determine the distribution with a reasonable degree of accuracy. Table 2.1 shows a typical example for a pool's structure distribution determined with different numbers of mutations. Each experiment was repeated 100 times, and the numbers shown represent the average and variance for each value. The averages are very stable for different numbers of mutations. However, the variance is clearly reduced with additional experiments, reaching very close to zero when the number of mutations reaches 1 million. Hence, 1 million mutations are expected to be sufficient to reliably estimate the structure distribution.

\begin{tabular}{|c|c|c|c|c|c|}
\hline $\begin{array}{c}\text { No. of } \\
\text { mutations }\end{array}$ & $\begin{array}{c}\text { 1J } \\
\text { structures(\%) }\end{array}$ & $\begin{array}{c}\text { 2J } \\
\text { structures(\%) }\end{array}$ & $\begin{array}{c}\text { 3J } \\
\text { structures(\%) }\end{array}$ & $\begin{array}{c}4 \mathrm{~J} \\
\text { structures(\%) }\end{array}$ & $\begin{array}{c}5 \mathrm{~J} \\
\text { structures(\%) }\end{array}$ \\
\hline $10^{2}$ & $10.04(9.72)$ & $49.45(30.23)$ & $27.12(20.19)$ & $8.78(9.71)$ & $4.61(4.54)$ \\
\hline $10^{3}$ & $10.26(0.70)$ & $49.99(3.05)$ & $26.45(2.04)$ & $8.44(0.65)$ & $4.86(0.41)$ \\
\hline $10^{4}$ & $10.31(0.09)$ & $49.87(0.29)$ & $26.69(0.19)$ & $8.34(0.07)$ & $4.80(0.04)$ \\
\hline $10^{5}$ & $10.36(0.01)$ & $49.93(0.02)$ & $26.57(0.02)$ & $8.36(0.01)$ & $4.78(0.00)$ \\
\hline $10^{6}$ & $10.35(0.00)$ & $49.93(0.00)$ & $26.58(0.00)$ & $8.35(0.00)$ & $4.79(0.00)$ \\
\hline
\end{tabular}

Table 2.1: Structural distribution determined with different numbers of mutations (averages and variances in brackets).

Secondary structure prediction was performed using the ViennaRNA 1.6.5 [64] software package in which RNAfold generates a single RNA/DNA secondary structure prediction through energy minimization based on a dynamic programming algorithm originally developed by Zuker and Stiegler [192]. ViennaRNA provides as output a nucleotide pairing list indicating for each nucleotide whether or not it is paired with another nucleotide, and if so to which. From this information we then determine the number of junctions in the RNA/DNA secondary structure using the CountJunctions algorithm.

The CountJunctions algorithm traverses the sequence from 5' to 3'. For each stem (at least four contiguous base pairs), there are two strands. Starting from one strand, if there is a stem along the path to another strand, we increase the junction number 
by 1. For example, in Figure 2.7, stem S1 has two strands, S1' and S1". Along the path from S1' to S1" in the 5' to 3' direction, each of the S2, S4, S5 stems forms one junction, so the number of junctions for stem $\mathrm{S} 1$ is 5 . For each stem, the number of junctions is at least 1 . The number of junctions for the RNA secondary structure is the maximum number of junctions of all stems. For example, if a secondary structure contains a 2-way and a 4-way junction (see Figure 2.14(b)), the secondary structure is defined as a 4-way junction structure.

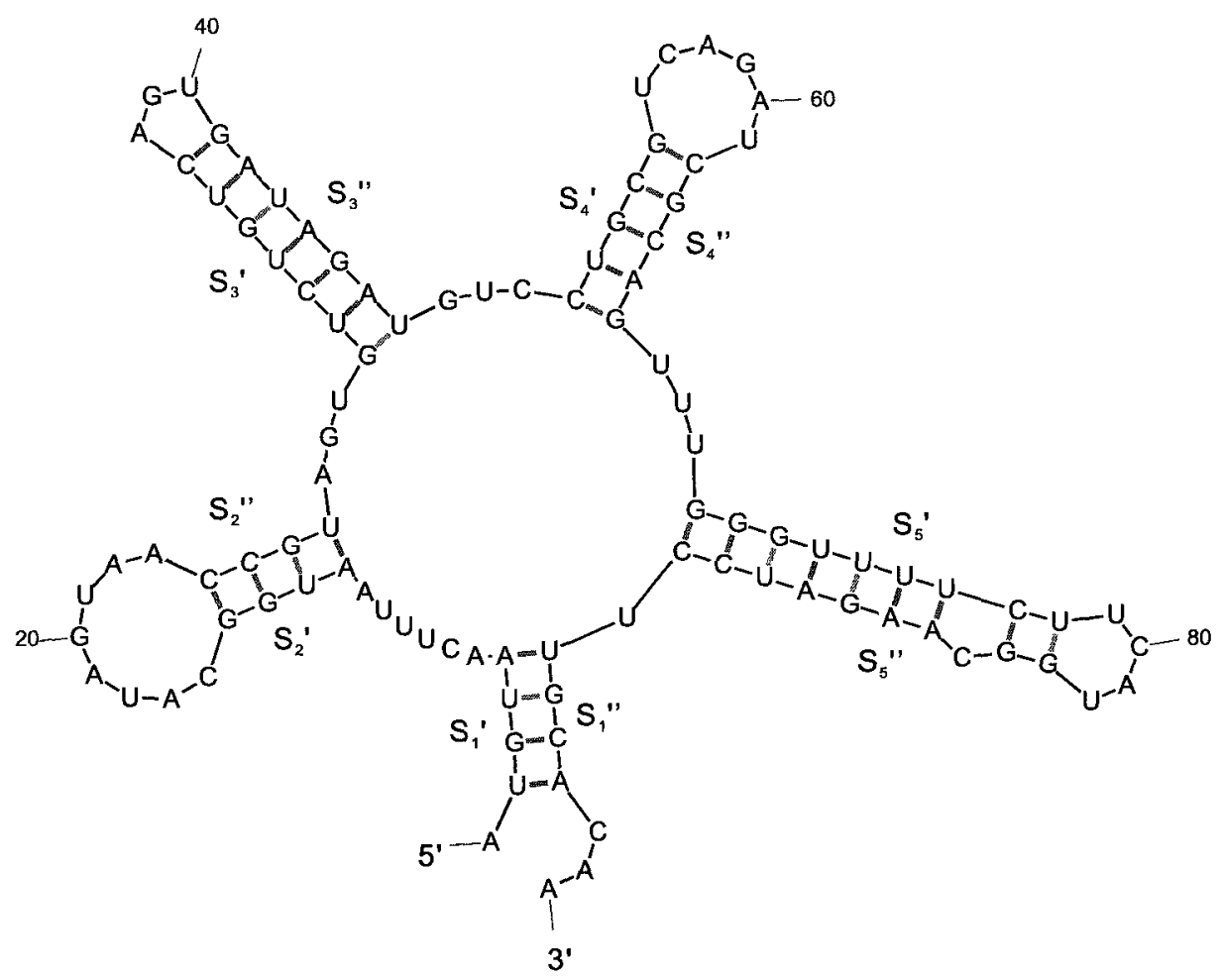

Figure 2.7: A sample 5-way junction structure used to calculate the method for counting junctions.

\section{Algorithm 1 CountJunctions}

(1) Scan the nucleotide pairing list created by ViennaRNA and determine the list $\left\{S_{1}, \ldots, S_{n}\right\}$ of all stems. We define a stem as a maximal list of at least four contiguous pairs.

(2) Consider the list $L$ of nucleotides from the 5' end to the 3' end. Each stem $S$ consists of two contiguous subsequences $S^{\prime}$ and $S^{\prime \prime}$ of $L$. Sort the list $L^{\prime}=$ $\left\{S_{1}^{\prime}, S_{1}^{\prime \prime}, S_{2}^{\prime}, S_{2}^{\prime \prime}, \ldots, S n^{\prime}, S n^{\prime \prime}\right\}$ according to their location in $L$ 
(3) For each stem, calculate its number of junction. A k-way junction consists of a sequence $S_{1}^{\prime}, S_{2}^{\prime}, S_{2}^{\prime \prime}, S_{3}^{\prime}, S_{3}^{\prime \prime}, \ldots, S_{k}^{\prime}, S_{k}^{\prime \prime}, S_{1}^{\prime \prime}$

(4) Find the maximum number of junctions

— End of Algorithm -

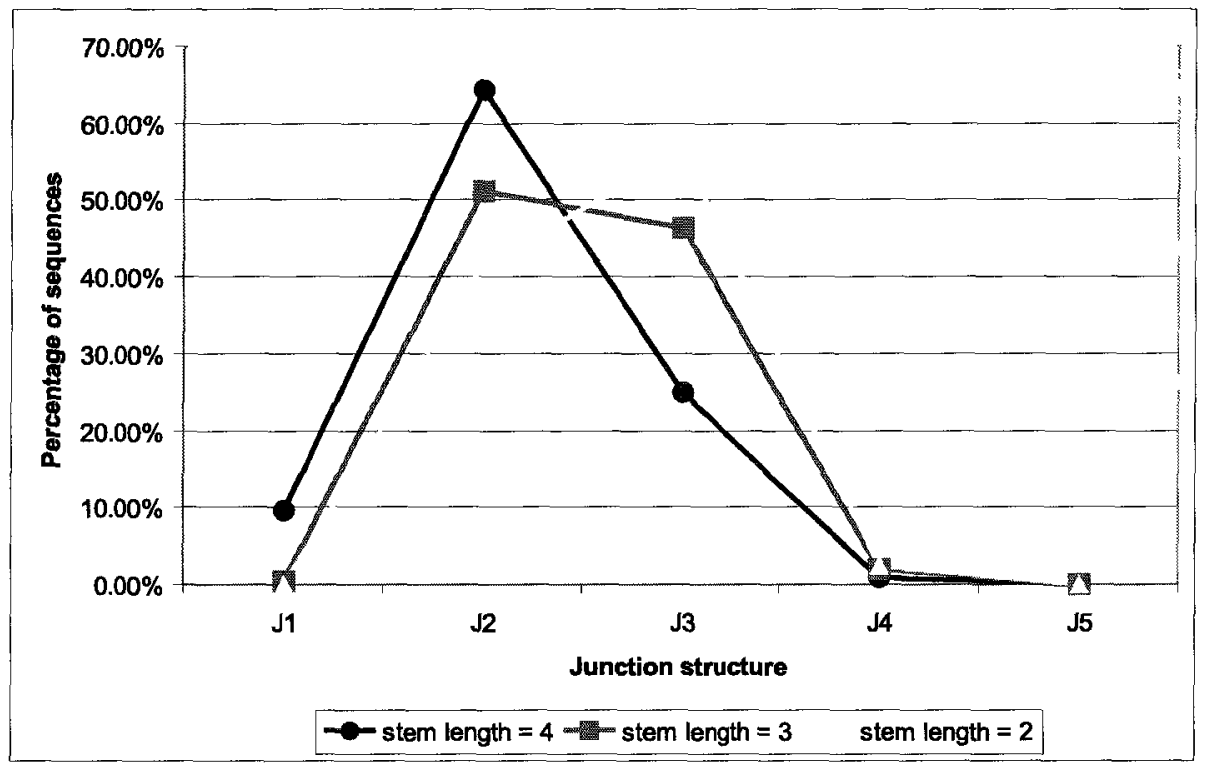

Figure 2.8: Comparison of junction distribution with different stem lengths. Selecting a smaller minimum stem length results in a larger number of predicted high complexity structures.

In Step 1 of Algorithm CountJunctions we define a stem as a maximal list of at least four contiguous pairs. We chose the most strict setting of minimum stem length $=4$ in accordance with [14]. The relationship between junction distribution count generated by Algorithm CountJunctions and minimum stem length is shown in Figure 2.8. We observed that selecting a smaller minimum stem length results in a larger number of predicted high complexity structures. However, the difference is not substantial.

\subsubsection{The Genetic Filtering method}

Genetic Filtering is a computational method to design diverse DNA pools for SELEX with uniform structure distribution, i.e. 20\% 1-way, 2-way, 3-way, 4-way and 5-way 
junctions each. A graphical overview of our method is given in Figure 2.9 and a more detailed Genetic Filtering pseudo-code is provided below (Algorithm 2).

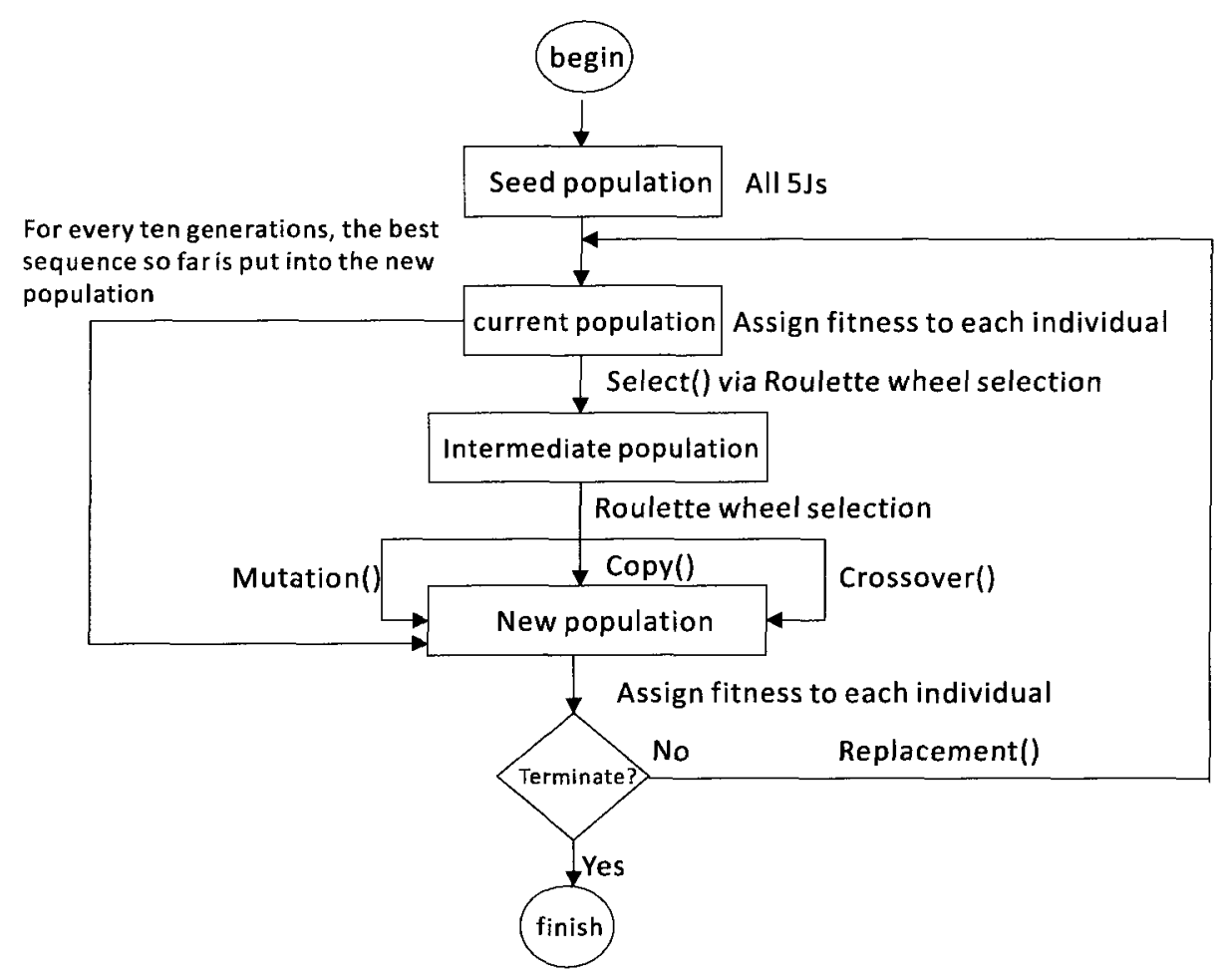

Figure 2.9: Graphical overview of the Genetic Filtering algorithm.

For a given pair of primers, Genetic Filtering first produces an initial generation of pool designs. Each pool design is assigned a fitness score which indicates its closeness to the desired uniform structure distribution. New generations of pool designs are then obtained by selecting designs from previous generations with better (i.e. smaller) fitness score and then applying three types of operations: mutation, copy and crossover. The initial generation of pool designs is based on a set of 5-way junctions which contain the given primers. The reason for selecting a set of 5-way junctions as the initial pool designs is that it is possible to obtain low complexity structures through the mutation of high complexity structures (see Figure 2.11) but the opposite is highly unlikely. This was also reported by a recent investigation [29] where 5Js were derived from the preexisting and less complex structures with 3Js through evolutionary pathways, but it was observed that the possibility is very small. 
A similar experiment is shown in Table 2.2. Mutating 50 different $3 \mathrm{~J}$ sequences 1 million times each, for a total of 50 million sequences, generated only $1625 \mathrm{~J}$ structures.

\begin{tabular}{|c|c|c|c|c|}
\hline $\begin{array}{c}\text { No. of 1J } \\
\text { structures }\end{array}$ & $\begin{array}{c}\text { No. of 2J } \\
\text { structures }\end{array}$ & $\begin{array}{c}\text { No. of 3J } \\
\text { structures }\end{array}$ & $\begin{array}{c}\text { No. of 4J } \\
\text { structures }\end{array}$ & $\begin{array}{c}\text { No. of 5J } \\
\text { structures }\end{array}$ \\
\hline 1498358 & 22284153 & 26031085 & 185724 & 162 \\
\hline
\end{tabular}

Table 2.2: Structural distribution of sequences created by mutating 50 different $3 \mathrm{~J}$ sequences 1 million times each.

Given an initial seed population of pool designs based on $5 \mathrm{~J}$ structures, our genetic algorithm proceeds as shown in Figure 2.9. Note that, all sequences (i.e. pool designs where all non-primer single-stranded positions are considered as random positions) always contain the given primers. Each sequence (pool design) is assigned a fitness score which indicates its closeness to the desired structure distribution. For a sequence $\mathrm{S}$, let $j c t_{\imath}$ be the percentage of i-way junctions counted after mutating its single stranded positions $10^{4}$ times, and let $d j c t_{\imath}$ be the desired percentage of i-way junctions $\{i=1, \ldots, 5\}$. The fitness score for $S$ is calculated as $\sum_{\imath=0}^{5}\left|j c t_{\imath}-d j c t_{\imath}\right|$. The desired percentage of i-way junctions is the uniform structure distribution and each $d j c t_{\imath}$ is set to $20 \%$. Note that, we perform $10^{4}$ instead of $10^{6}$ mutations of single stranded positions for the fitness score calculations. The number of mutations is reduced for fitness score estimation to save computation time. As discussed in Section 2.4, our Genetic Filtering method takes hours on a 200 processor PC cluster. As shown in Table 2.1, the structure distribution estimated with $10^{4}$ mutations is close to that estimated with $10^{6}$ mutations. Furthermore, we confirm our final result by re-calculating the fitness score with $10^{6}$ mutations.

We further modified the fitness score for pool designs in Genetic Faltering such that designs without a desired number of single stranded (random) positions were penalized. When each sequence (pool design) is assigned a fitness score, we penalize sequences whose number of single stranded positions (num_ss) is not equal to the desired number of single stranded positions (dnum_ss), and less penalty is given to pool designs with fewer single stranded positions. For details see Algorithm 3. In our case, we chose 24 as the ideal number of single stranded positions. The reason for this lies in the SELEX experiment. Most SELEX experiments begin with a starting pool 
consisting of nanomoles of sequences, as this is a reasonable amount of DNA that can be synthesized. This translates to approximately $10^{14}$ to $10^{15}$ sequences. Given that 4 possible bases can be inserted at any random sequence position and $10^{15} \approx 4^{25}$, this implies a target number of random positions in the pool design of approximately 25 is sufficient for the entire sequence space to be sampled once in a given experiment. Here, we chose to include 24 random positions in our pool design to ensure complete sequence coverage in our starting pool (i.e. allowing four expected copies for every different sequence).

After the fitness scores have been calculated for the current population of pool designs, biased selection (based on fitness scores) is applied to select a new intermediate population of pool designs with better fitness. Here, we use a roulette wheel selection method to select pool designs from the current population where each pool design is chosen with a probability that corresponds to its relative fitness (see Algorithm 4). By repeatedly spinning the roulette wheel, a new intermediate population is selected and fitter individuals have a greater chance to be selected than weaker ones.

Crossover, mutation and copy are then applied to the intermediate population to create the next generation. For the crossover method, we first choose two sequences $\mathrm{S} 1$ and S2 from the intermediate population of pool designs, and then generate a random number called randomPosition between two parameters Low and High. To create the next generation, we switch the sub-sequences of S1 and S2 from position randomPosition to position length - randomPosition, where length is the total length of each sequence. The crossover method is designed to potentially switch substructures between two sequences. This is clearly not always the case, but when it happens, new pool designs of interest can be generated. When a sequence in the intermediate population is selected for mutation, we generate a random number between 0 and 1 for each position of the sequence excluding primers. If the number is less than a parameter $P_{\text {position, }}$, we mutate the position by choosing with equal likelihood one of the other three nucleotides (see Algorithm 5). The mutated sequence will then enter the next generation. When a sequence in the intermediate population is selected for a copy operation, the sequence is simply copied unchanged to the next generation. The above crossover, mutation and copy methods have parameters 
$P_{\text {crossover }}, P_{\text {mutation }}$ and $P_{\text {copy }}$, respectively, which represent the likelihood of applying the respective method to a given sequence. Furthermore, for every 10 generations we re-add the best sequence found so far to the new generation of pool designs.

Genetıc Faltering generates a number of generations of pool designs (typically between 500 and 3000) untıl either a pool design with uniform structure distribution is found, or the score of the best pool design remains unchanged for a number of generations, or a maximum number of generations $(N G)$ is reached.

\section{Algorithm 2 Genetıc Falterıng Algorithm (Pseudo-Code)}

initialize currentGeneratıon [popSıze]

for i from 0 to popsize

$$
\begin{aligned}
& \text { distance }[\imath]=\sum_{\imath=0}^{5}|\jmath c t[\imath]-\operatorname{d\jmath ct}[\imath]| \\
& \text { fıtness }[\imath]=\text { fitness_with_penalty }()
\end{aligned}
$$

endfor

Fitness $=\sum$ fitness $[\imath]$

for $\mathrm{i}$ from 0 to popSize

$$
\begin{aligned}
& \operatorname{prob} F[\imath]=1-\text { finess }[\imath] / \text { Futness } \\
& \operatorname{prob} C[\imath]=\sum_{\jmath=0}^{\imath} \operatorname{probF}[\jmath]
\end{aligned}
$$

endfor

num_generation $=0$

for each i from 0 to popSize

$$
\begin{aligned}
& \text { oldGeneratıon }[\imath]=\text { currentGeneration }[\imath] \\
& \text { oldFutness }[\imath]=\text { fitness }[\imath]
\end{aligned}
$$

endfor

while minFitness $\neq 0$ and num_generatıon $\leq N G$ and NoChangeCounter $<$ NoChangeCutoff

$$
\mathrm{j}=0
$$

while $\jmath<$ popSize

if num generation $\% 10=0$

$$
\text { newGeneratıon }[0]=\text { bestSequence }
$$




$$
\begin{aligned}
& \text { fitness }[0]=\text { best_fitness } \\
& \mathrm{j}=\mathrm{j}+1
\end{aligned}
$$

endif

$P_{\text {random }}=$ random number from $[0,1]$

if $0 \leq P_{\text {random }}<P_{\text {crossover }}$

seq1=roulette_wheel_select()

seq $2=$ roulette_wheel_select()

midGeneration, midGeneration $2=\operatorname{crossover}(\operatorname{seq} 1$, seq2)

if midGeneration, midgeneration2 not in newGeneration

newGeneration $[j]=$ midGeneration

newGeneration $[j+1]=$ midGeneration 2

$$
\mathrm{j}=\mathrm{j}+2
$$

endif

else if $P_{\text {crossover }} \leq P_{\text {random }}<P_{\text {crossover }}+P_{\text {mutation }}$

seq $=$ roulette_wheel_select()

midGeneration $=$ mutate_positions $(\mathrm{seq})$

if midGeneration not in newGeneration

$$
\text { newGeneration }[j]=\text { midGeneration }
$$

$$
\mathrm{j}=\mathrm{j}+1
$$

endif

else

midGeneration=roulette_wheel_select()

if midGeneration not in newGeneration

$$
\begin{aligned}
& \text { newGeneration }[j]=\text { midGeneration } \\
& \mathrm{j}=\mathrm{j}+1
\end{aligned}
$$

endif

endif

endwhile

replace(currentGeneration, newGenration)

for each i from i to popSize 
if currentGeneration [i $]$ in oldGeneration

$\mathrm{ix}=$ index of currentGeneration $[i]$ in oldGeneration

fitness $[i]=$ oldFitness $[i x]$

else

$\operatorname{distance}[i]=\sum_{i=0}^{5}|j c t[i]-\operatorname{djct}[i]|$

fitness $[i]=$ fitness_with_penalty ()

oldGeneration [size_oldGeneration $]=$ currentGeneration $[i]$

oldFitness[size_oldGeneration $]=$ fitness $[i]$

size_oldGeneration=size_oldGeneration +1

endif

endfor

update Fitness, $\operatorname{probF}[i]$, probC $[i]$

num_generation $=$ num_generation +1

bestSequence $=$ MAX(bestSequence_newGeneration, bestSequence_current)

if bestSequence has improved

NoChangeCounter $=0$

else NoChangeCounter $=$ NoChangeCounter +1

endif

endwhile

— End of Algorithm -

Algorithm 3 Method: fitness_with_penalty

num_ss $=$ number of ss positions

dnum_ss $=$ the desired number of ss positions

if num_ss $\geq$ dnum_ss

fitness $=$ distance $+0.2^{*}$ (num_ss-dnum_ss)

else fitness $=$ distance $+($ dnum_ss-num_ss $)$.

endif

- End of Algorithm - 
Algorithm 4 Method: Roulette_wheel_selection

$\mathrm{r}=$ random percentage number from $[0,1]$

if $r=\operatorname{prob} C[0]$

return currentGeneration [0]

else if $\operatorname{prob} C[k-1]<r \leq \operatorname{prob} C[k]$

return currentGeneration [k]

endif

— End of Algorithm -

Algorithm 5 Method: mutate_positions

$R N A[]=\{$ "A", "G", "C", "T" $\}$

for position i from primer_length to (length - primer_length-1)

$\mathrm{r}=$ random percentage number from $[0,1]$

if $r<P_{\text {position }}$

$\mathrm{n}=$ random number from $[0,3]$

while sequence[i] $=$ RNA[n]

$\mathrm{n}=$ random number from $[0,3]$

endwhile

sequence $[\mathrm{i}]=\mathrm{RNA}[\mathrm{n}]$

endif

endfor

— End of Algorithm -

\subsubsection{The $R N A$ To Tree method}

RNA To Tree is a computational method to convert RNA structures to tree graphs. It uses the structure information generated by the CountJunctions algorithm and the tree graph rules defined in Section 2.2.5 to convert a RNA secondary structure into a tree graph. RNA To Tree proceeds as follows: we first use step 1 of the CountJunctions algorithm (see Algorithm 1) to compute the total number of stems. Note that according to tree graph rule 4 defined in Section 2.2.5, the minimum length 
of a stem structure was defined as at least two contiguous pairs here. Based on step 2 and 3 of the CountJunctions algorithm, we then use the ConvertingRNAToTree algorithm to build a tree graph shown below (Algorithm 6).

Similar to the CountJunctions algorithm, the ConvertingRNAToTree algorithm traverses the sequence from 5' to 3'. As tree graph rule 3 defined in Section 2.2.5, the 3 '- and 5'-ends of a helical stem are considered as a vertex. We assume that 5'-end links to the first stem, so we name 5'-end as vertex B0 and add an edge between vertices B0 and B1. For each stem $i$, along the path from its one strand to its another strand, if there is a stem $\mathrm{j}$ that forms a junction, we add edge between vertices $\mathrm{B}(\mathrm{i})$ and $\mathrm{B}(\mathrm{j})$. For example, in Figure 2.5, along the path from $S_{1}^{\prime}$ to $S_{1}^{\prime \prime}$ in the 5' to 3' direction, each of the $S_{2}, S_{3}, S_{4} S_{5}$ stems forms one junction, so we add edges between B1 and B2, B3, B4, B5 respectively. Note that stem $S_{6}$ does not contribute a junction for stem $S_{1}$, but it forms one junction along the path from $S_{5}^{\prime}$ to $S_{5}^{\prime \prime}$, so we add an edge between B5 and B6, not between B1 and B6. In the ConvertingRNAToTree algorithm, endIndex represents the index number of the stem which links to 3'-end. If endIndex is not 0, that means 3'- and 5'-ends link to different stems (see Figure 2.12), so we name 3'-end as vertex B(endIndex) and add an edge between vertices $\mathrm{B} 0$ and $\mathrm{B}$ (endIndex).

\section{Algorithm 6 ConvertingRNAToTree}

$\mathrm{n}=$ number of stems

endIndex $=0$

add edge between vertices B0 and B1

for stem $\mathrm{i}$ from 1 to $\mathrm{n}$

if stem i links to 3 ' end

endIndex $=\mathrm{i}$

endif

for stem $\mathrm{j}$ from 1 to $\mathrm{n}$

if stem $\mathrm{j}$ forms one junction for stem $\mathrm{i}$

add edge between vertices $B(i)$ and $B(j)$

endif 
endfor

endfor

if endIndex $>0$

add edge between vertices $\mathrm{B} 0$ and $\mathrm{B}$ (endIndex)

endif

— End of Algorithm

\subsection{Results}

\subsubsection{Increasing the number of complex structures in SELEX starting pools (Random Filtering method)}

Using Vienna RNA to fold one million 100nt random sequences, we identified $765 \mathrm{~J}$ sequences. These sequences were subjected to Random Filtering to generate a 5J enhanced pool. Figure 2.10 shows an increase in the structural complexity (using junction order) of a pool generated from Random Filtering (using the 76 5Js as a starting point) as compared to a pool of 1 million 100 nt random sequences. The most frequent structures in the Random Filtering pools are 3Js $(48.65 \%)$ while the most frequent structures in the random pools are 2Js (64.35\%). In the Random Filtering pools, $10.23 \%$ of the sequences are $5 \mathrm{Js}$ and $15.45 \%$ of the sequences are $4 \mathrm{Js}$, compared with $0.01 \% 5 \mathrm{Js}$ and $0.95 \%$ J $\mathrm{Js}$ in the random pools.

Since primer sites (short-fixed sequences at the 5'- and 3'- ends required for amplification) are generally used in SELEX experiments, we examined the effect of including them on structural diversity. We used a $1 \overrightarrow{5} \mathrm{nt} 5$ ' primer site "GGAAGAGATGGCGAC" and a 15nt 3' primer site "AGCTGATCCTGATGG", thereby occupying 30 fixed positions in our starting sequence. This leaves only 70 nt that can be modified for the design of the starting pool, decreasing the sequence length from 100, making it considerably more difficult to find complex structures [147].

From a 100nt pool of 20 million random sequences containing these primers, we obtained only 35 sequences exhibiting $5 \mathrm{Js}$. These sequences were used to generate a pool using Random Filtering. Compared with 76 5Js found in a 1 million 100nt random pool without primers, $5 \mathrm{Js}$ are 40 folds less abundant in a $100 \mathrm{nt}$ pool containing 


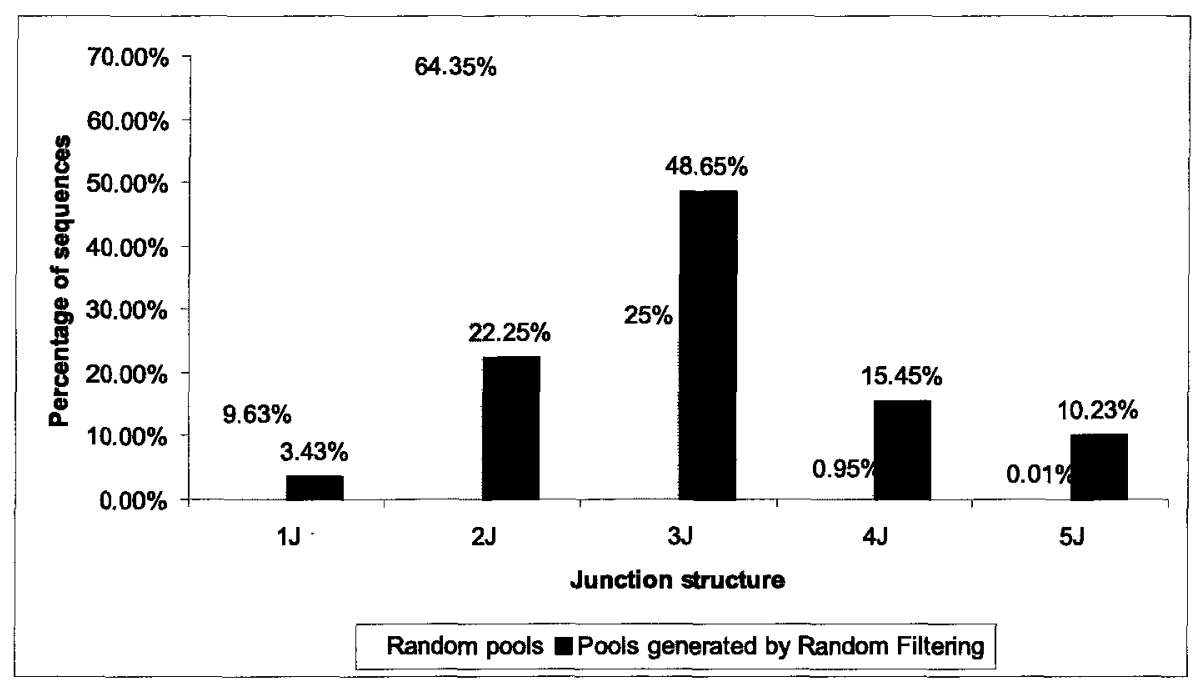

Figure 2.10: Comparison of average structural distribution of 1 million sequences for random RNA/DNA pools and RNA/DNA pools generated by Random Filtering. Random Filtering has greatly increased the percentage of high complex structures such as $5 \mathrm{~J}_{\mathrm{s}}$ (by $10 \%$ ) and $4 \mathrm{Js}$ (by $15 \%$ ).

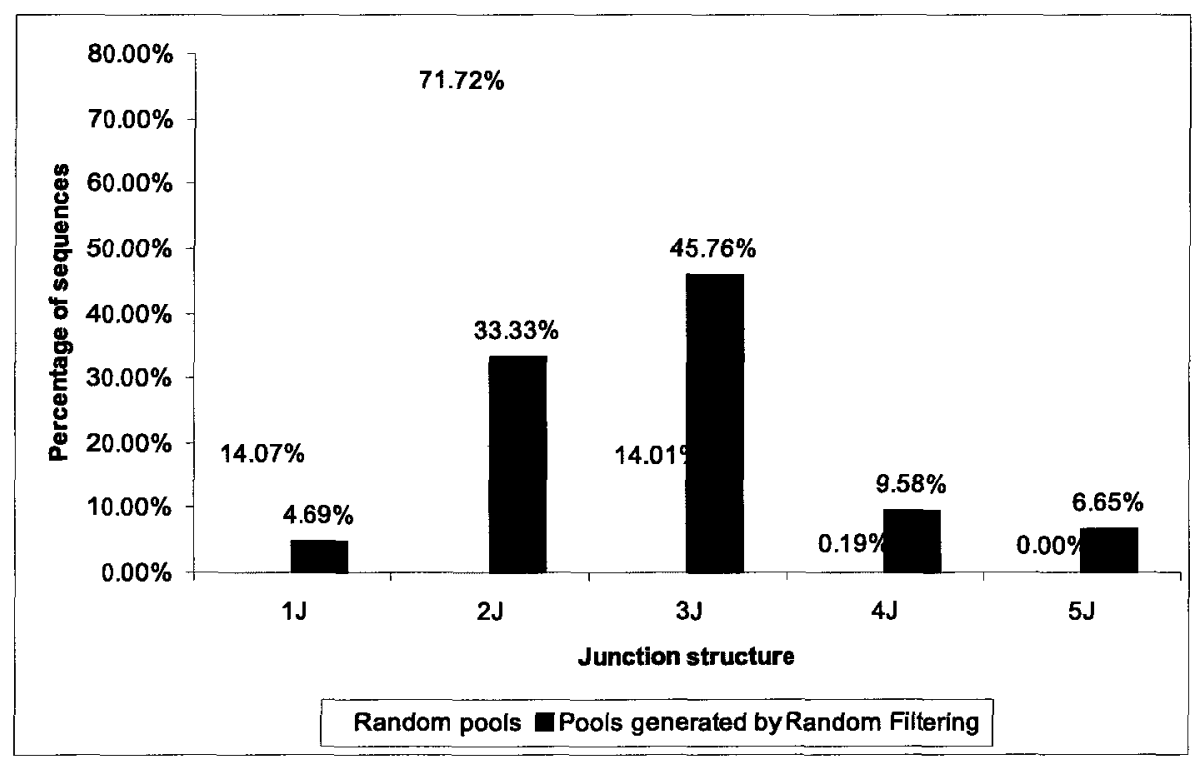

Figure 2.11: Comparison of average structural distribution of 1 million sequences for random RNA/DNA pools and RNA/DNA pools generated by Random Filtering with 15nt primers at both ends, "GGAAGAGATGGCGAC" and "AGCTGATCCTGATGG". Random Filtering has greatly increased the percentage of high complex structures such as 5Js (by 6\%) and 4Js (by 10\%). 
30nt fixed primers. According to Sabeti et al. [147], a 5J motif in a 70nt random pool is about 100 to 200 fold less abundant than a $5 \mathrm{~J}$ motif in a $100 \mathrm{nt}$ random pool. However, primers can participate in the formation of junctions even though the effective pool length has been shortened. Therefore, the 5Js abundances in a 100nt pool containing 30nt primers should be higher than a simple 70nt random pool. Similar to the case without primers, Random Filtering produces a more structurally complex pool (see Figure 2.11). The most frequent structures in our Random Filtering pools are $3 \mathrm{~J}$ sequences $(45.76 \%$ ) while the most frequent structures in random pools are 2J (71.72\%). In Random Filtering pools, $6.65 \%$ of the sequences are $5 \mathrm{Js}$ and $9.58 \%$ of the sequences are $4 \mathrm{Js}$, compared with $0.00 \% 5 \mathrm{Js}$ and $0.19 \% 4 \mathrm{Js}$ in random pools. Therefore, Random Filtering increases the percentage of highly complex structures available for selection in the SELEX experiments. The sequence shown in Figure 2.12 called RFPool $A$ has 19 single stranded positions (indicated as "N") excluding primers and resulted in the largest number of $5 \mathrm{Js}(31.13 \%)$ (Table 2.3 ).

\begin{tabular}{|c|c|c|c|c|c|c|}
\hline & $\begin{array}{c}\text { 1J } \\
\text { Structures }\end{array}$ & $\begin{array}{c}2 \mathrm{~J} \\
\text { Structures }\end{array}$ & $\begin{array}{c}3 \mathrm{~J} \\
\text { Structures }\end{array}$ & $\begin{array}{c}4 \mathrm{~J} \\
\text { Structures }\end{array}$ & $\begin{array}{c}5 \mathrm{~J} \\
\text { Structures }\end{array}$ & $\begin{array}{c}\text { No. of } \\
\text { SS pos. }\end{array}$ \\
\hline in silico & $3.28 \%$ & $25.68 \%$ & $24.68 \%$ & $15.22 \%$ & $31.13 \%$ & 19 \\
\hline in vitro & $0 \%$ & $47.06 \%$ & $29.41 \%$ & $11.76 \%$ & $11.76 \%$ & - \\
\hline
\end{tabular}

Table 2.3: in silico and in vitro structural distribution for RFPool $A$.

The RFPool A was synthesized, amplified, cloned and sequenced and these sample sequences were folded using ViennaRNA. Of the 17 sequences recovered, $11.76 \%$ of them formed 5-way junctions (see Table 2.3), the structure alignments are shown in Figure 2.13.

It may initially appear that the structural diversity of a pool resulting from our filtering approaches will be overly constrained given the stretches of fixed positions in the stems. However, an examination of a number of structures resulting from the same pool design demonstrates that while the sequence in these regions is constrained, the corresponding structure is not. Examples of different junction structures formed from the RFPool $A$ are shown in Figure 2.14. These sample structures illustrate that the same fixed sequence region may participate in a stem in one structure while forming a loop in another. For example, in the 5J structure shown in Figure 2.14(a), 


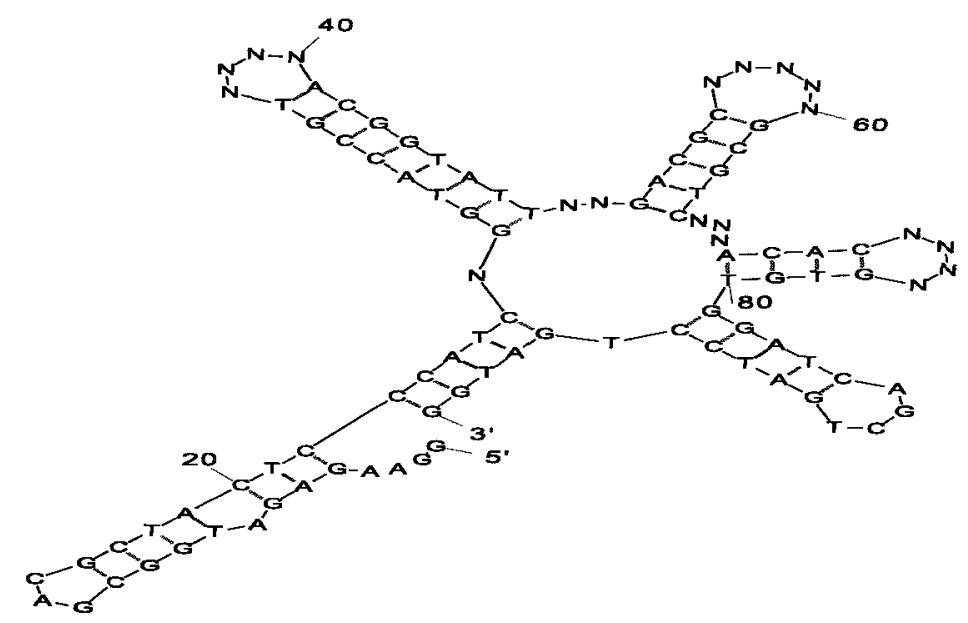

Figure 2.12: Sample secondary structure of RFPool $A$. RFPool $A$ including primers "GGAAGAGATGGCGAC" and "AGCTGATCCTGATGG" was designed to generate the highest percentage of $5 \mathrm{~J}$ sequences. The sequence has 19 single stranded positions excluding primers (noted as ' $\mathrm{N}$ '). As a starting pool for SELEX experiments, these 19 single stranded positions are considered as random $(\mathrm{N})$ and the other positions are considered as fixed.

the sequence GCGT starting at base 61 participates in a stem while the same fixed sequence forms a loop in the corresponding $4 \mathrm{~J}$ structure illustrated in Figure 2.14(b).

For our Random Filtering method to work, several 5-way junctions need to be initially found by repeatedly examining random sequences; these $5 \mathrm{Js}$ serve as the starting point for the Random Filtering method. The ability to generate 5Js depends primarily on the sequence length [147], whereas the number of 5Js obtained also depends on the size of the pool. Figure 2.15 shows the relationship between sequence length and the number of $5 \mathrm{Js}$ that can be found for one million random sequences with and without primer regions. The numbers shown are consistent with, but not equal to, the estimates given in Sabeti et al. [147]; see discussion below. As the pool sequence length decreases, the number of $5 \mathrm{Js}$ in the random pool decreases as well. When the sequence length is decreased to 76 , no $5 \mathrm{Js}$ were found in the random pool. This means that when $5 \mathrm{~J}$ sequences are needed in a 1-million random pool without 


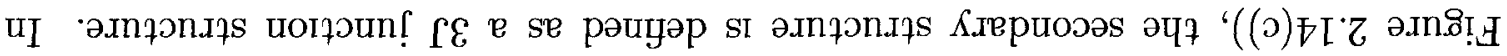

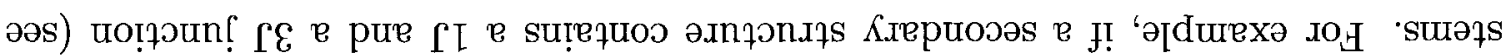

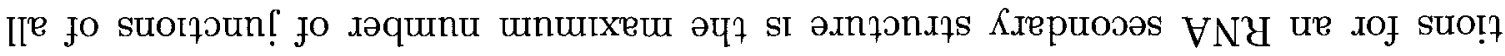

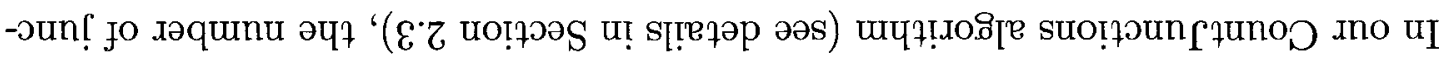

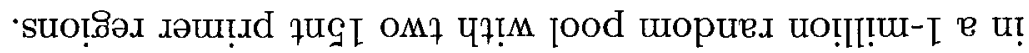

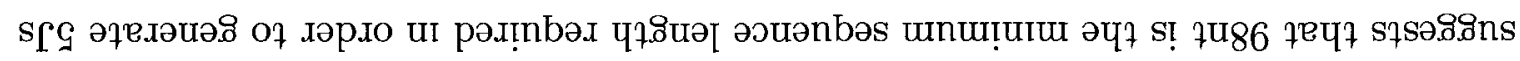

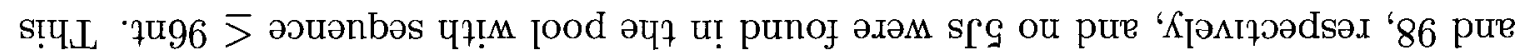

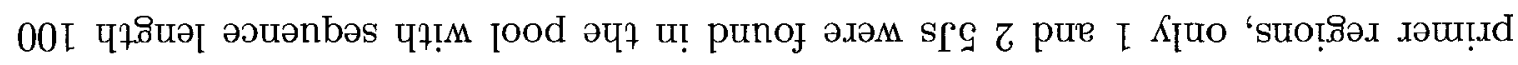

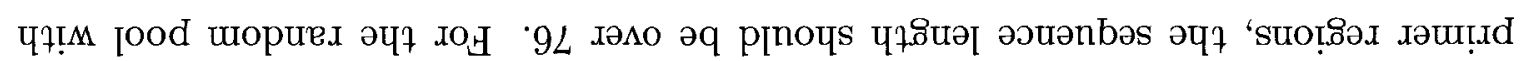

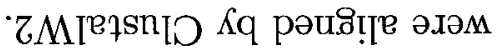

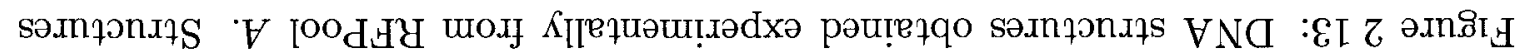

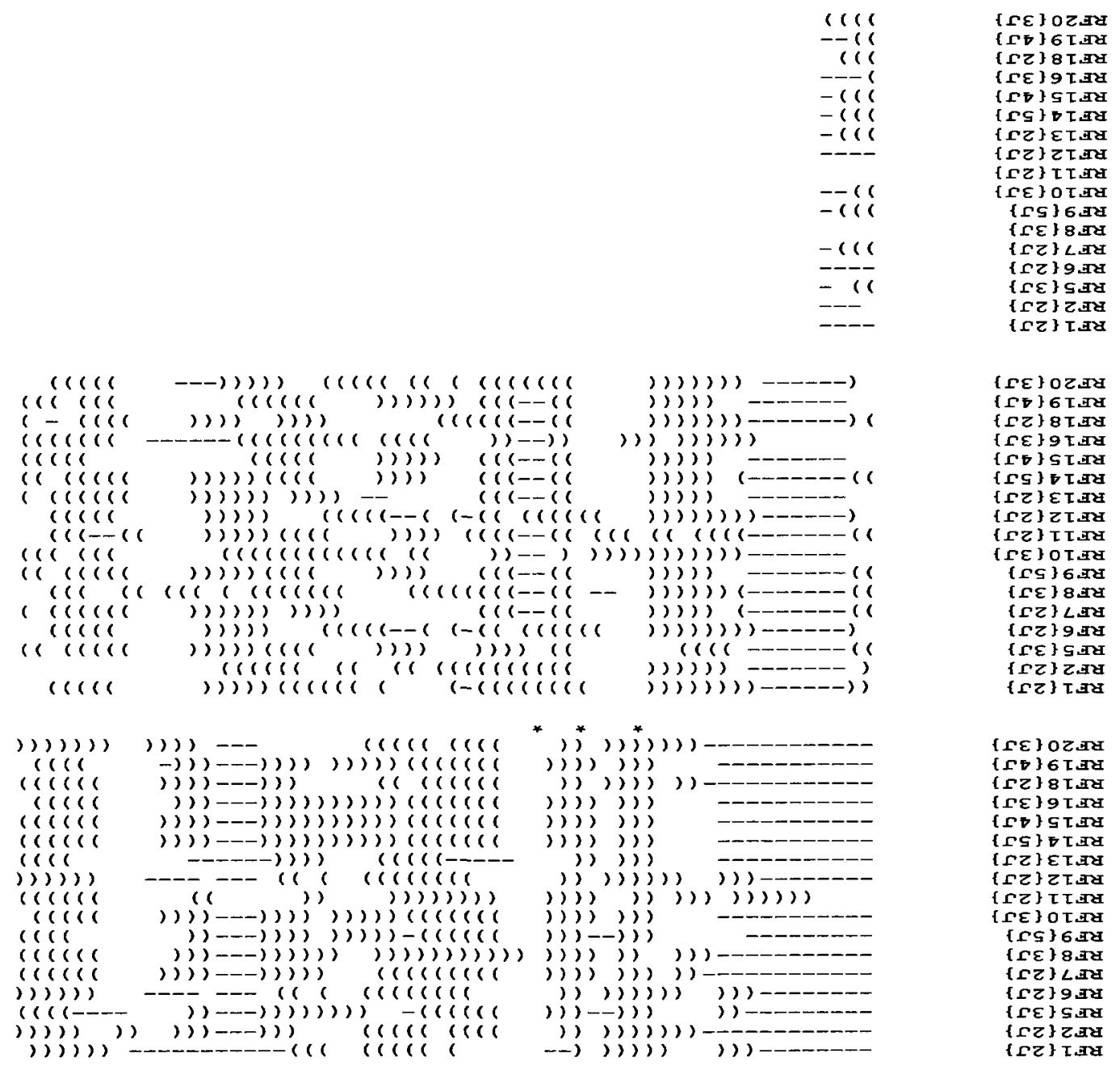




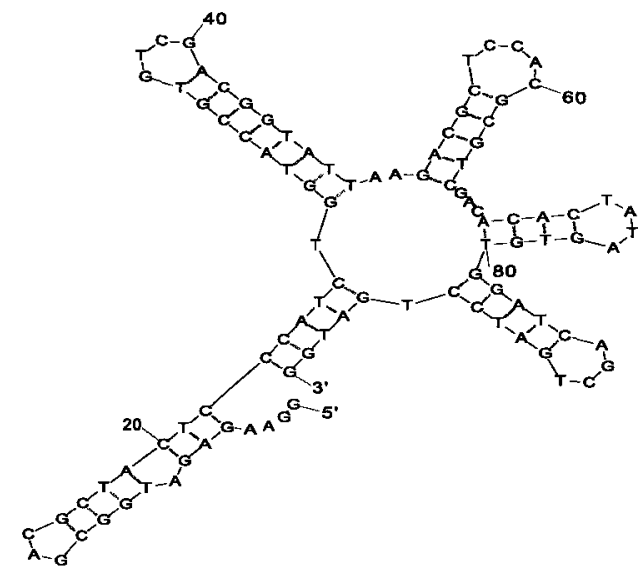

(a) a $5 \mathrm{~J}$ structure

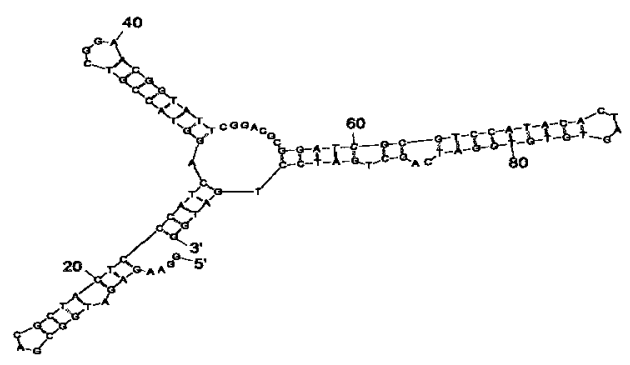

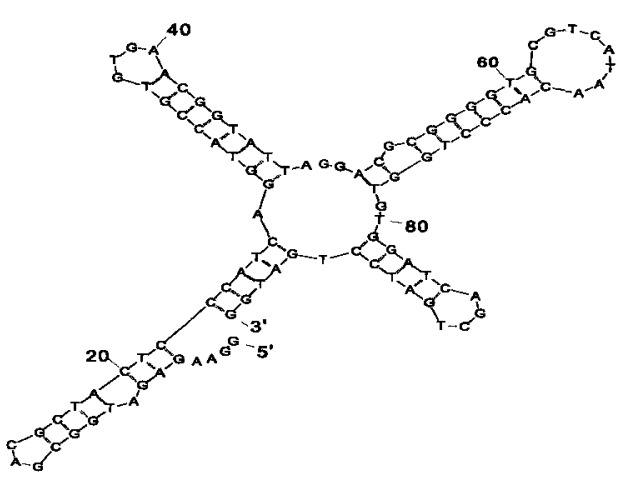

(b) a $4 \mathrm{~J}$ structure

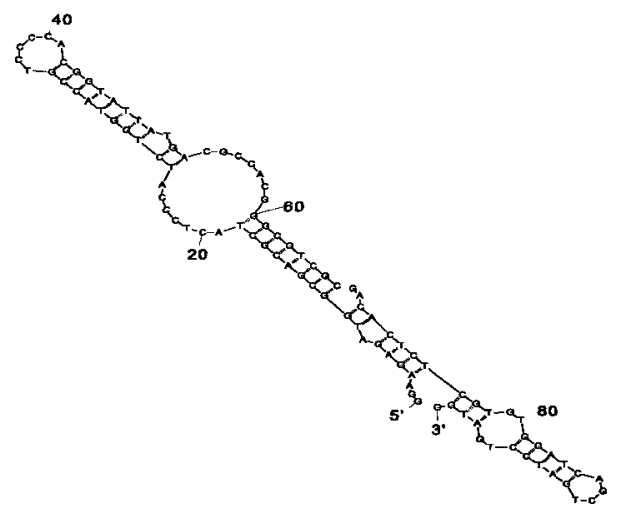

(c) a $3 \mathrm{~J}$ structure

(d) a $2 \mathrm{~J}$ structure

Figure 2.14: Sample sequences and representative secondary structures for RFPool $A$.

contrast, Sabeti et al. [147] calculated the probability of finding a particular motif within a random sequence. That is, their method would count the sequence in Figure 2.14 (c) as having a $1 \mathrm{~J}$ and $3 \mathrm{~J}$ motif. Therefore, the percentages of n-way junction 


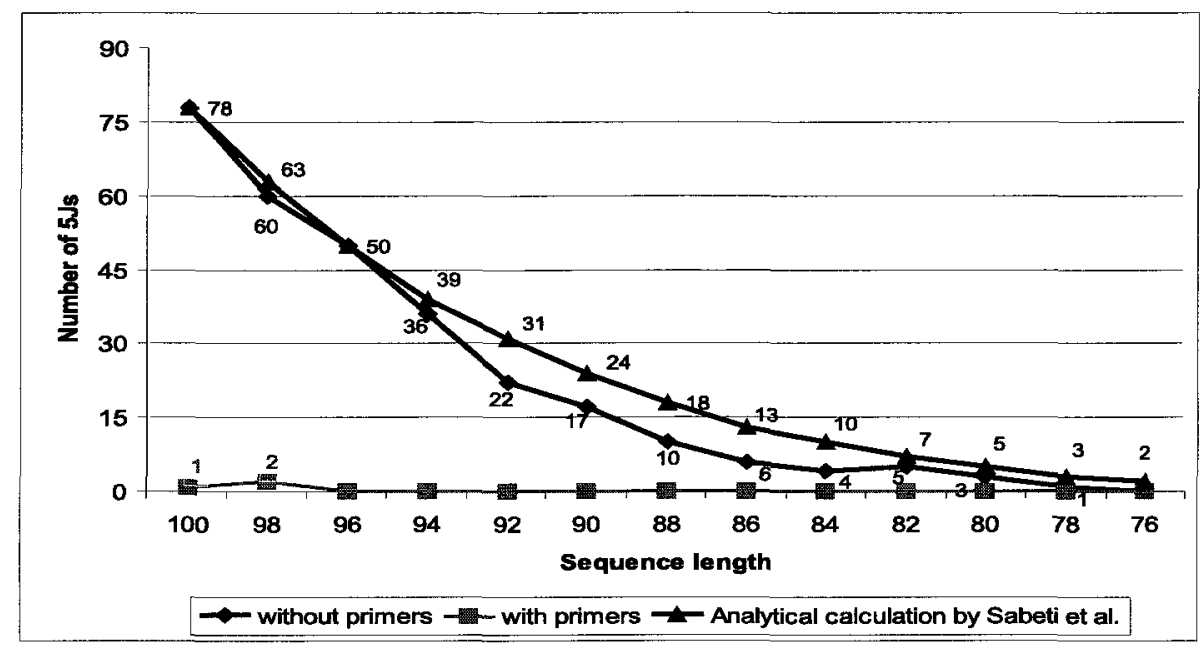

Figure 2.15: Relationship between 5J population and sequence lengths for a random pool of 1 million sequences with and without primers. When $5 \mathrm{~J}$ sequences are needed in a 1-million random pool without primer regions, the sequence length should be over 76. In order to generate $5 \mathrm{Js}$ in a 1-million random pool with two $15 \mathrm{nt}$ primer regions, $98 \mathrm{nt}$ is the minimum sequence length required. Analytical calculation is based on Equation 2 in Sabeti et al. [147], given $n \approx 56$. The "without primer" curve is in direct agreement with this analytical calculation.

in a random pool calculated by our CountJunction method are different from those of Sabeti et al. Since $5 \mathrm{~J}$ is the most complex structure found for 100nt random pools in our computation, a sequence containing a $5 \mathrm{~J}$ structure is less likely to contain other motifs. Therefore, the $5 \mathrm{~J}$ abundance calculated by our CountJunction method should be close to those found by Sabeti et al. For example, 78 J Js are found in a 100nt random pool without the primer regions (see Figure 2.15). Based on this starting point, 63,50 and $395 \mathrm{Js}$ can be found for 98, 96 and 94nt pools, respectively, according to Equation 2 in Sabeti et al. [147], given $\mathrm{n}=56$ (see Figure 2.15). The "without primer" curve in Figure 2.15 is in direct agreement with this analytical calculation.

\subsubsection{Designing a diverse SELEX starting pool with uniform structural distribution (Genetic Filtering method)}

In the previous section we showed how the Random Filtering method enriches a pool with complex structures such as 5-way junctions. Here, we also present a method, termed Genetic Filtering, to design a diverse starting pool for in vitro selection with 
a uniform structure distribution, i.e. a pool with 20 percent of each $1 \mathrm{~J}, 2 \mathrm{~J}, 3 \mathrm{~J}, 4 \mathrm{~J}$ and $5 \mathrm{~J}$ structures.

We ran our Genetic Filtering method on a 100nt pool design with primer sites "ATACCAGCTTATTCAATT" and "AGATAGTAAGTGCAATCT" for 571 generations with a runtime of $2 \mathrm{hrs} 10 \mathrm{~min}$ on a 200 processor cluster. The best fitness score decreases steadily with each generation until it remains steady for approximately 500 generations (Figure 2.16). The distribution is close to a uniform distribution (Table 2.4) with a distance from the optimal of 2.95. The best pool design, GFPool1, is shown in Figure 2.17. This pool was also examined experimentally to confirm its structural diversity after 8 rounds of SELEX.

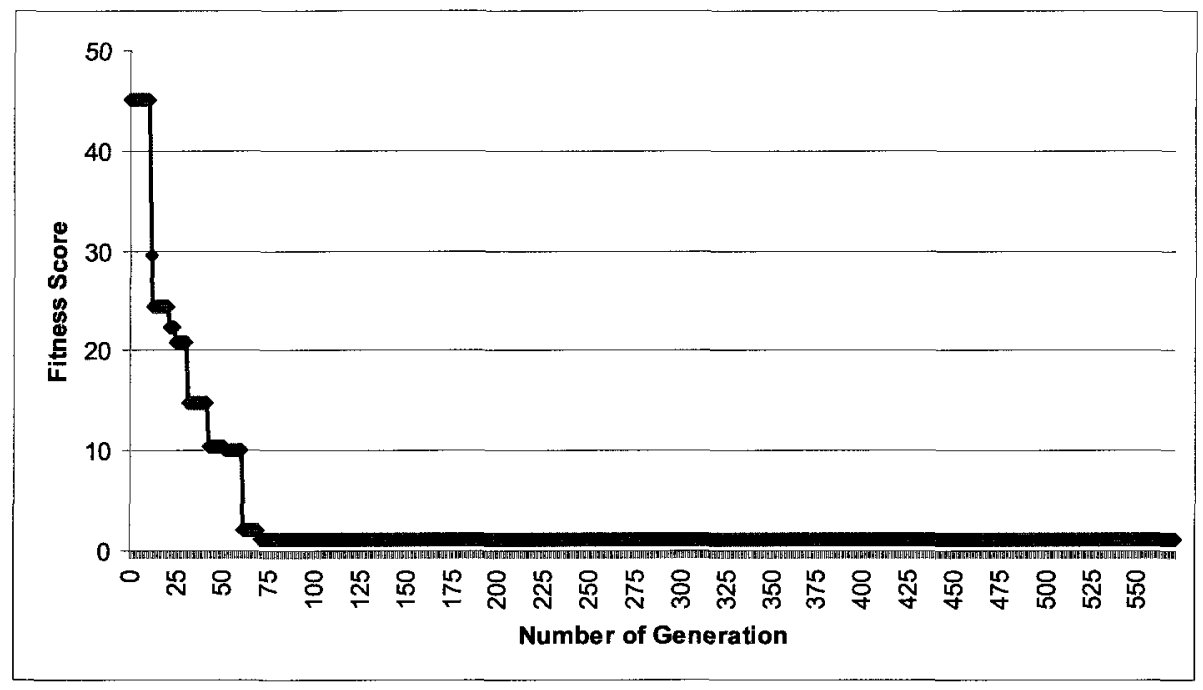

Figure 2.16: The best fitness value observed so far at each generation of Genetic Filtering (primers "ATACCAGCTTATTCAATT" and "AGATAGTAAGTGCAATCT"). Parameters: $\mathrm{NG}=10000$ NoChargeCutoff $=500$ Pcrossover $=0.0$ Pmutation $=0.9$ Pposition $=0.0156$ Pcopy $=0.1$ (see Section 2.3.2). The best fitness score (smallest value), which indicates its closeness to the desired uniform structure distribution, decreases steadily with each generation until it remains steady for approximately 500 generations.

\begin{tabular}{|c|c|c|c|c|c|c|}
\hline $\begin{array}{c}1 \mathrm{~J} \\
\text { Structures }\end{array}$ & $\begin{array}{c}2 \mathrm{~J} \\
\text { Structures }\end{array}$ & $\begin{array}{c}\text { 3J } \\
\text { Structures }\end{array}$ & $\begin{array}{c}4 \mathrm{~J} \\
\text { Structures }\end{array}$ & $\begin{array}{c}5 \mathrm{~J} \\
\text { Structures }\end{array}$ & Distance & $\begin{array}{c}\text { No. of } \\
\text { SS pos. }\end{array}$ \\
\hline $19.59 \%$ & $20.37 \%$ & $18.93 \%$ & $20.42 \%$ & $20.69 \%$ & 2.95 & 24 \\
\hline
\end{tabular}

Table 2.4: Structural distribution for GFPool1. 


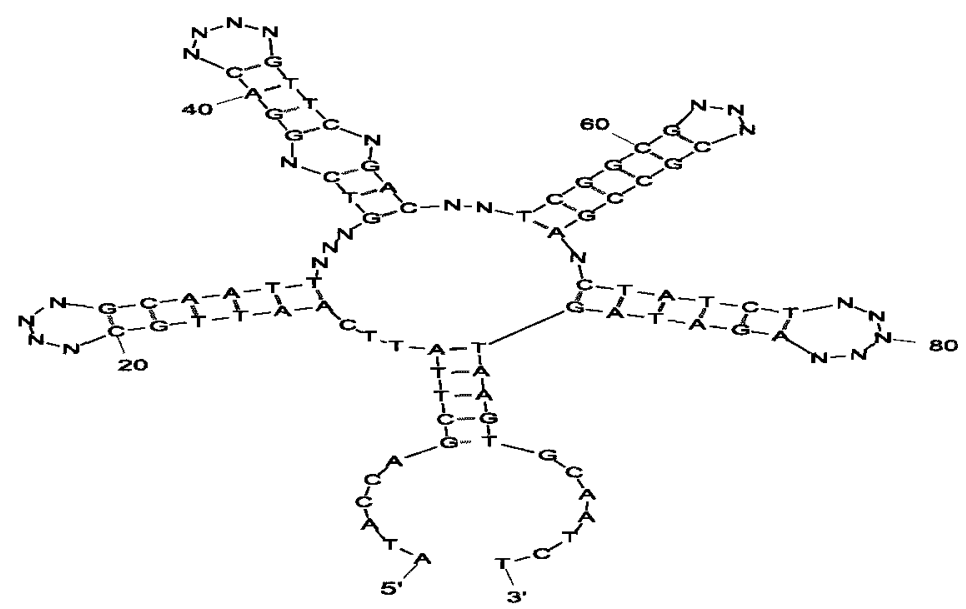

Figure 2.17: Sample secondary structure of GFPool1. GFPool1 is the DNA pool sequence including primers "ATACCAGCTTATTCAATT" and "AGATAGTAAGTGCAATCT". This was designed to generate a SELEX starting pool with uniform structure distribution. This 100nt in length pool, containing 24 random nucleotide positions, was synthesized and used in selection experiments for binding ATP.

The effect of primer site and number of single stranded positions was also investigated for the Genetic Faltering approach. Using a 5' primer sequence "ATACCAGCTTATTCAATT" and 3' primer sequence "AGATTGCACTTACTATCT", Genetic Filtering stopped at generation 1626, and the runtime on a 200 processor PC cluster was $6 \mathrm{hrs} 17 \mathrm{~min}$. Figure 2.18 shows the best (smallest) fitness score of the pool designs observed so far at each generation. The best pool design, GFPool2 (Figure 2.19) delivered a structure distribution (Table 2.5) close to the uniform structure distribution, having a deviation of 4.93. The number of single stranded positions in the best pool design was 25, despite having a penalty in the fitness function for deviations from the ideal 24 positions. In order to obtain a pool design with uniform structural distribution and exactly 24 random positions, we performed the following additional computation. For each single stranded position, excluding primers, we kept that single stranded position fixed and mutated the other 24 single stranded positions 


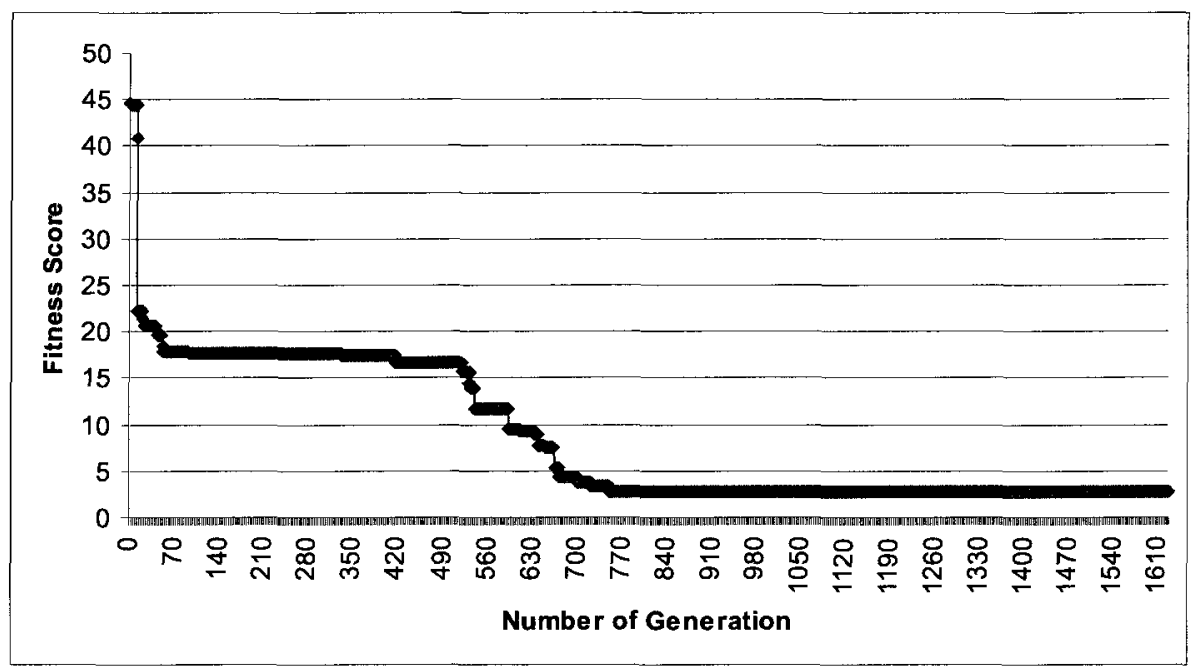

Figure 2.18: The best fitness value observed at each generation of Genetic Filtering (primers "ATACCAGCTTATTCAATT" and "AGATTGCACTTACTATCT"). Parameters: $\mathrm{NG}=10000$ NoChargeCutoff $=500$ Pcrossover $=0.0$ Pmutation $=0.9$ Pposition $=0.0156$ Pcopy $=0.1$ (see Section 2.3.2). The best fitness score decreases steadily with each generation and stopped at generation 1626 when it remains steady after approximately 500 generations.

$10^{6}$ times, calculating the structural distribution for those $10^{6}$ mutations. The best distribution was obtained when the 20th single stranded position was kept fixed, in which case the distribution is nearly equal to the optimal uniform structural distribution (see Table 2.5). The pool design, GFPool2A, obtained by Genetic Filtering with very close to uniform structural distribution and exactly 24 random positions is "ATACCAGCTTATTCAATTAAGCNNNNNGTCCACNNNNNNGTGGATNGCCCCCNNNNGGNNGGGCNNTGGCTNNNNGCCAGGTAGATTGCACTTACTATCT".

\begin{tabular}{|c|c|c|c|c|c|c|c|}
\hline & 1Js & 2Js & 3Js & 4Js & 5Js & Distance & SS pos. \\
\hline GFPool2 & $18.74 \%$ & $19.73 \%$ & $19.06 \%$ & $22.10 \%$ & $20.36 \%$ & 4.93 & 25 \\
\hline GFPool2A & $19.08 \%$ & $20.14 \%$ & $19.93 \%$ & $20.29 \%$ & $20.56 \%$ & 1.97 & 24 \\
\hline
\end{tabular}

Table 2.5: Structural distribution, distance from the optimal, and number of single stranded positions present for GFPool2 and GFPool2A. GFPool2: Pool shown in Figure 2.19 using primers "ATACCAGCTTATTCAATT" and 3' prier "AGATTGCACTTACTATCT" GFPool2A: Pool 2 modified by keeping the 20th single stranded position fixed. 


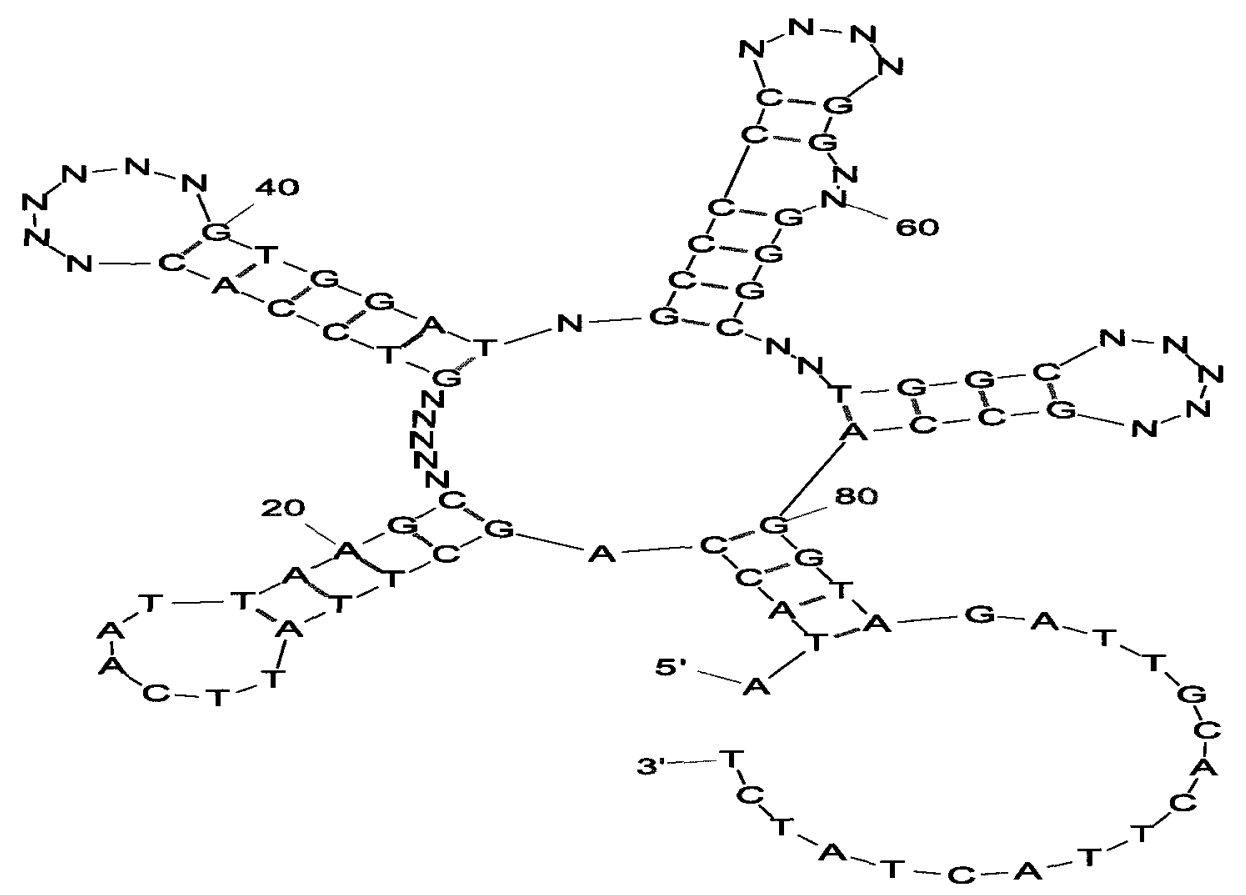

Figure 2.19: Sample secondary structure of GFPool2. GFPool2 uses primers "ATACCAGCTTATTCAATT" and "AGATTGCACTTACTATCT". It was designed to generate a SELEX starting pool with uniform structure distribution. This pool is $100 \mathrm{nt}$ in length and contains 25 random nucleotide positions.

\subsubsection{Designing a SELEX starting pool with uniform tree structure dis- tribution}

Similar to the method for designing the uniform junction distribution pool, we designed a pool with a uniform distribution of 12 tree structures from 21 to $6_{5}$ in Figure 2.6. We started from a random pool and used ViennaRNA to generate the secondary structures, then converted the secondary structures to the tree structures using the RNA To Tree method. In order to compare with the mixing matrices approach $[86,88]$, the minimum length of a stem structure was defined as at least two contiguous pairs, and pools of 10,000 sequences were used for computation. Each tree structure is classified into different structure categories. Given an initial seed population of pool designs based on a number of tree structures such as $5_{3}, 6_{1}, 6_{2}$, $6_{3}, 6_{4}$ and $6_{5}$, we used Genetic Filtering to create a single design that would yield a startup pool with a uniform distribution of 12 tree structures. The pool design obtained by this experiment (GFPool3) and its structural distribution are shown in 
Figure 2.20 and Figure 2.21, respectively.

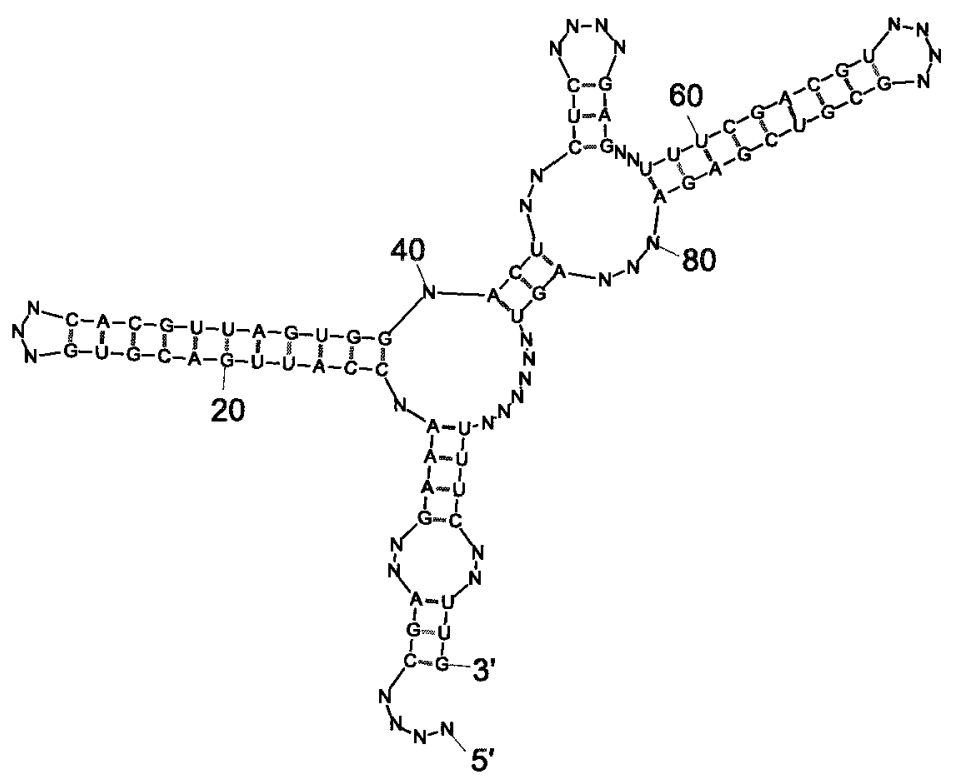

Figure 2.20: Sample secondary structure of GFPool3. GFPool3 is the DNA pool sequence designed to generate a SELEX starting pool with uniform tree structure distribution.

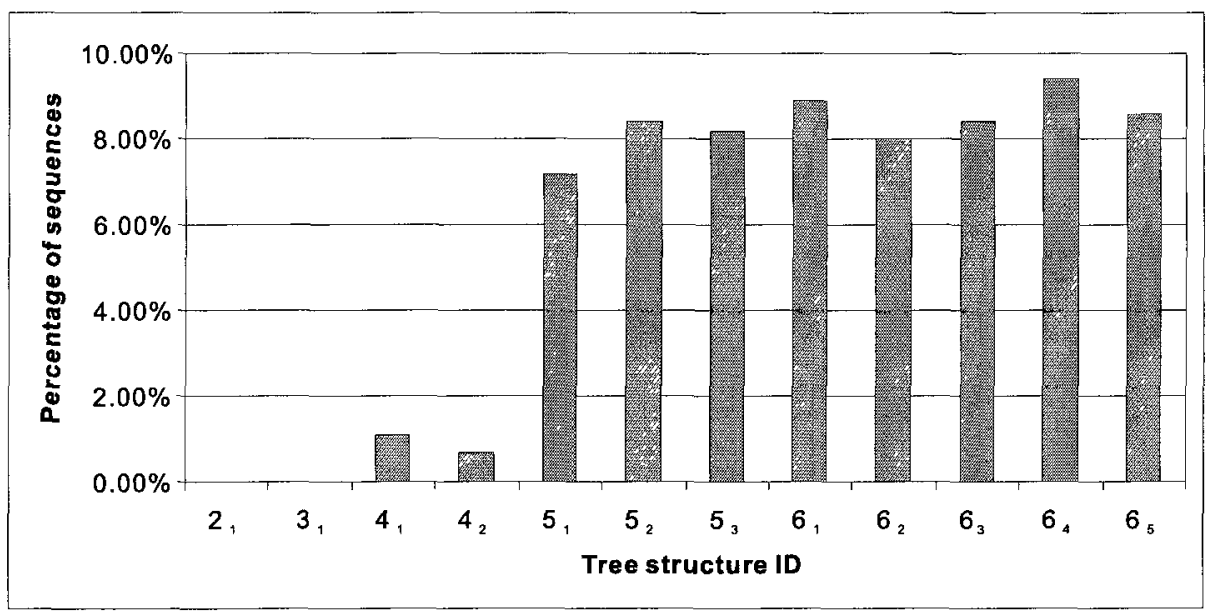

Figure 2.21: Tree structure distribution of GFPool3. 


\subsubsection{Result of SELEX experiment with improved pool design}

SELEX experiments were performed using our uniform structure pool, GFPool1, generated by our Genetıc Filtering method, in an effort to confirm that our design approach yielded a pool that allowed for an increased range of complex structures to be sampled during the selection process. Selections were performed to find DNA sequences that showed affinity for ATP [72]. In the original ATP aptamer SELEX experiments, the random DNA pool used to screen for ATP binders consisted of $2 \times 10^{14}$ different sequences made up of a 72 nucleotide random region flanked by defined primer binding sites. After 8 rounds of selection, the random region of the sequence with the best binding affinity was determined to be 5'-GACTG GGCTT GTGCT TGGGG GAGTA TTGCG GAGGA AAGCG GCCCT GCTGA AGTGG GATAC ATGTG GATAC CC -3' (personal communication, J.W. Szostak). This sequence, with its flanking primers, is of low complexity, predicted to form a $2 \mathrm{~J}$.

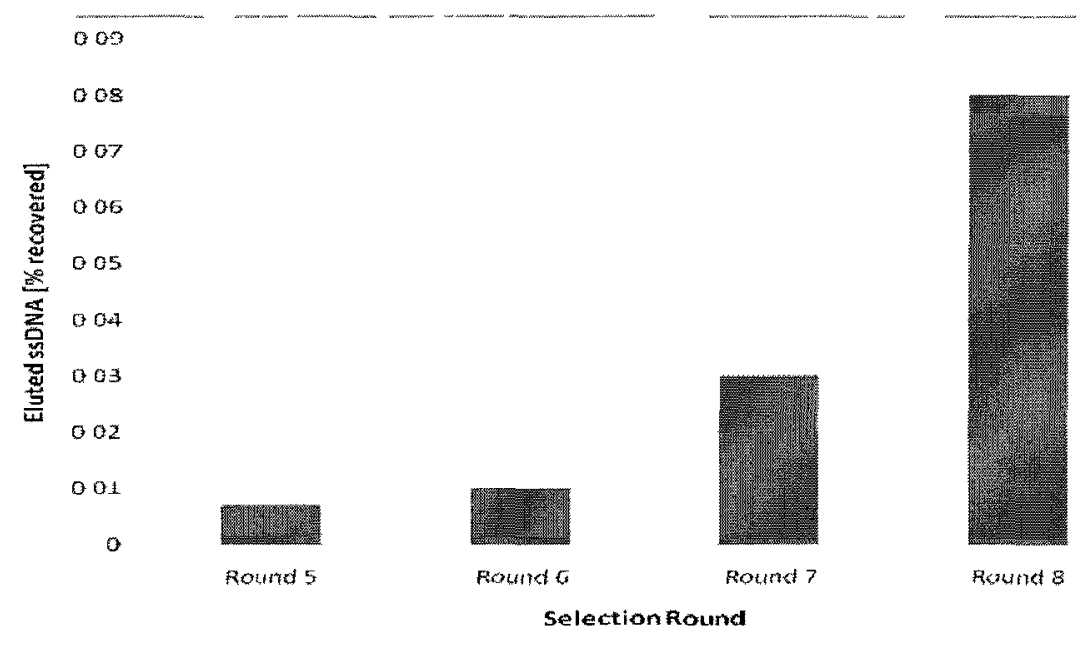

Figure 2.22: Percent recovery of DNA eluted from the ATP selection column versus selection round. With each round, an increased percentage of the pool DNA bound to the target was observed.

Given the extensive study of this system, we chose ATP as the target for testing our uniform structure pool, GFPool1, generated by our Genetic Filtering method. A total of eight rounds of SELEX were performed, equivalent to the original procedure by Szostak. The recovery of DNA after each round, corresponding to the amount of DNA binding to the ATP target, was monitored using UV-vis absorbance and 
fluorescence measurements. With each round, an increased percentage of the pool DNA bound to the target was observed (see Figure 2.22). DNA from GFPool1 prior to selections as well from the round 8 enriched pool was cloned and sequenced. The secondary structures were analysed using Vienna RNA. Table 2.6 shows the distribution of junction structures obtained for both the starting and enriched pools, and structures can be found in Figure 2.23 and Figure 2.24, where structures were aligned by ClustalW2. Of the 19 sequences obtained experimentally from the non-enriched starting pool, $1 \mathrm{~J}_{\mathrm{s}}, 2 \mathrm{~J}_{\mathrm{s}}, 3 \mathrm{~J}_{\mathrm{s}}, 4 \mathrm{Js}_{\mathrm{s}}$ and $5 \mathrm{~J}_{\mathrm{s}}$ were found. Additionally, 2Js, 3Js and $5 \mathrm{~J}_{\mathrm{s}}$ were found in the enriched pool, confirming not only the structural diversity of this designed pool, but also that complex structures were retained after several rounds of enrichment.

\begin{tabular}{|c|c|c|c|c|c|c|c|}
\hline & $1 \mathrm{Js}$ & $2 \mathrm{Js}$ & $3 \mathrm{Js}$ & $4 \mathrm{Js}$ & $5 \mathrm{Js}$ & Distance & SS pos. \\
\hline in silico & $19.6 \%$ & $20.4 \%$ & $18.9 \%$ & $20.4 \%$ & $20.7 \%$ & 2.95 & 24 \\
\hline $\begin{array}{c}\text { in vitro } \\
\text { (before enrichment) }\end{array}$ & $15.8 \%$ & $26.3 \%$ & $10.5 \%$ & $21.1 \%$ & $26.3 \%$ & - & - \\
\hline $\begin{array}{c}\text { in vitro } \\
\text { (after 8 rounds of SELEX) }\end{array}$ & $0 \%$ & $50 \%$ & $30 \%$ & $0 \%$ & $20 \%$ & - & - \\
\hline
\end{tabular}

Table 2.6: in silico structural distribution, distance from the optimal, and number of single stranded positions present for DNA pools with primers as well as experimental structural distributions prior to starting SELEX and after 8 rounds of selection using ATP as the target. GFPool1: Pool shown in Figure 2.17 using primers "ATACCAGCTTATTCAATT" and "AGATAGTAAGTGCAATCT".

Two of the 5J sequences, ATPF4 and ATPF8 (see Figure 2.24), were tested for binding affinity to ATP, yielding dissociation constant $K d$ values of $24.4 \mu M$ and $3.7 \mu M$, respectively. In comparison, a $K d$ of $6 \mu M$ is reported for the published ATP aptamer sequence [72]. It is important to note that the published ATP aptamer sequence underwent extensive optimization and minimization prior to binding affinity experiments. The fact that one 5J generated from our SELEX experiments shows improved binding over that optimized system, while the other $5 \mathrm{~J}$ does not, suggests that complexity alone does not guarantee better target binding, but higher complexity structures do have the potential to yield better aptamers. This clearly underscores the need for structural diversity, not just structural complexity, in the initial starting pool. 


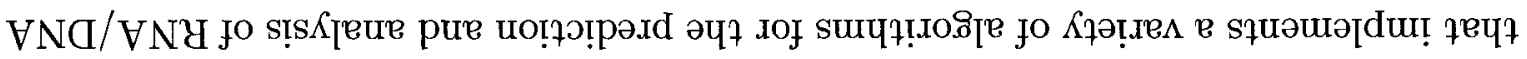

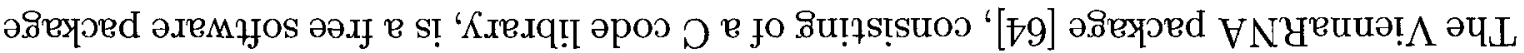

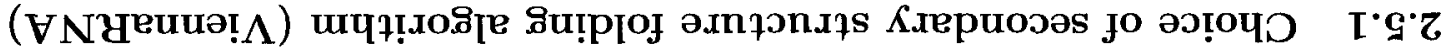

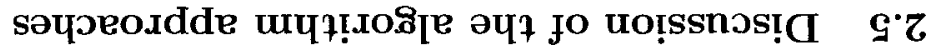

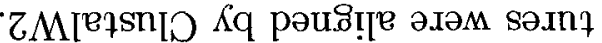

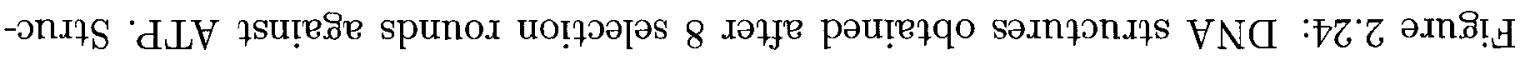

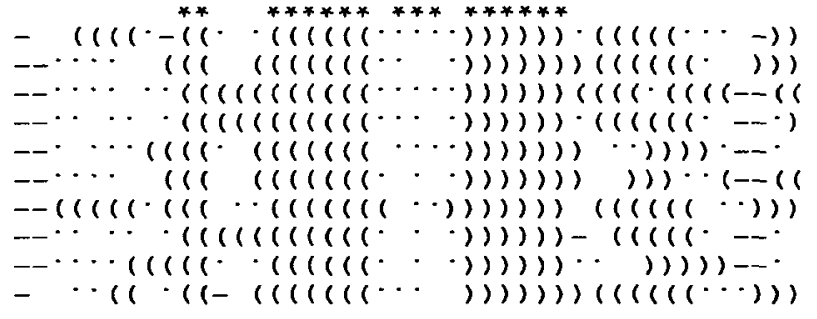

)$)(\cdots)--())),) \cdots{ }^{*} \cdots\left(\left(\left(\left(\left(\left(-\left(\left(\left(\left((((\cdots)))^{* * *}\right)\right)\right) \cdots \cdot\right)\right)\right)\right)\right)\right)^{* *} \cdot\right.$

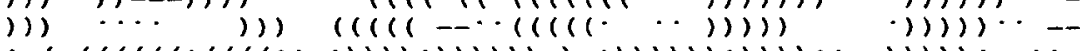

- $(((((((\cdot((((\cdots \cdot)))) \cdot))))))), \cdot)))))) \cdot)))) \cdots())))) \cdots$

$1)))) \cdots((((((((\cdot-))))))))) \cdot((((((\cdots)))))) \cdots))))) \cdots \cdot$

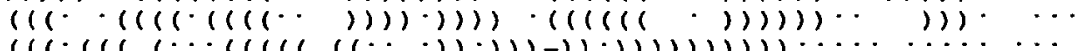

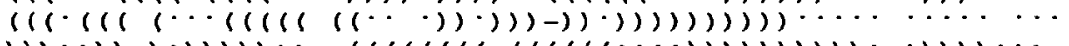

1) $\cdots))(\cdot))))) \cdots((((((((1(((((\cdots)))))))))) \cdot \cdot \cdot)))) \cdots \cdot$

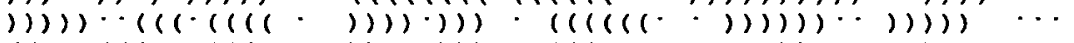

$(((-((((((-(((1-2))))))))))((((((-\cdots)))))) \cdots \cdots))) \cdots$

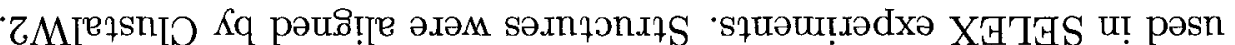

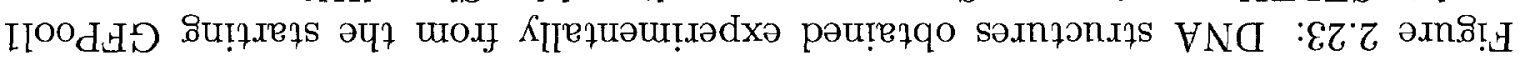

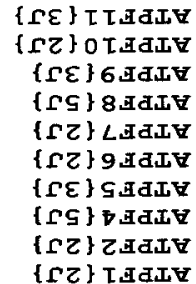

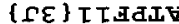
\{rz\} 0 โ जUdW \{ $ᄃ$ \} 6 ज्ञाय \{rS\} 8JALV $\{\rho z\} \angle A d L V$ \{ᄃZ\} 9. 저

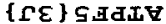
\{rS\} TुaLV $\{\rho Z\} 乙$ IdU

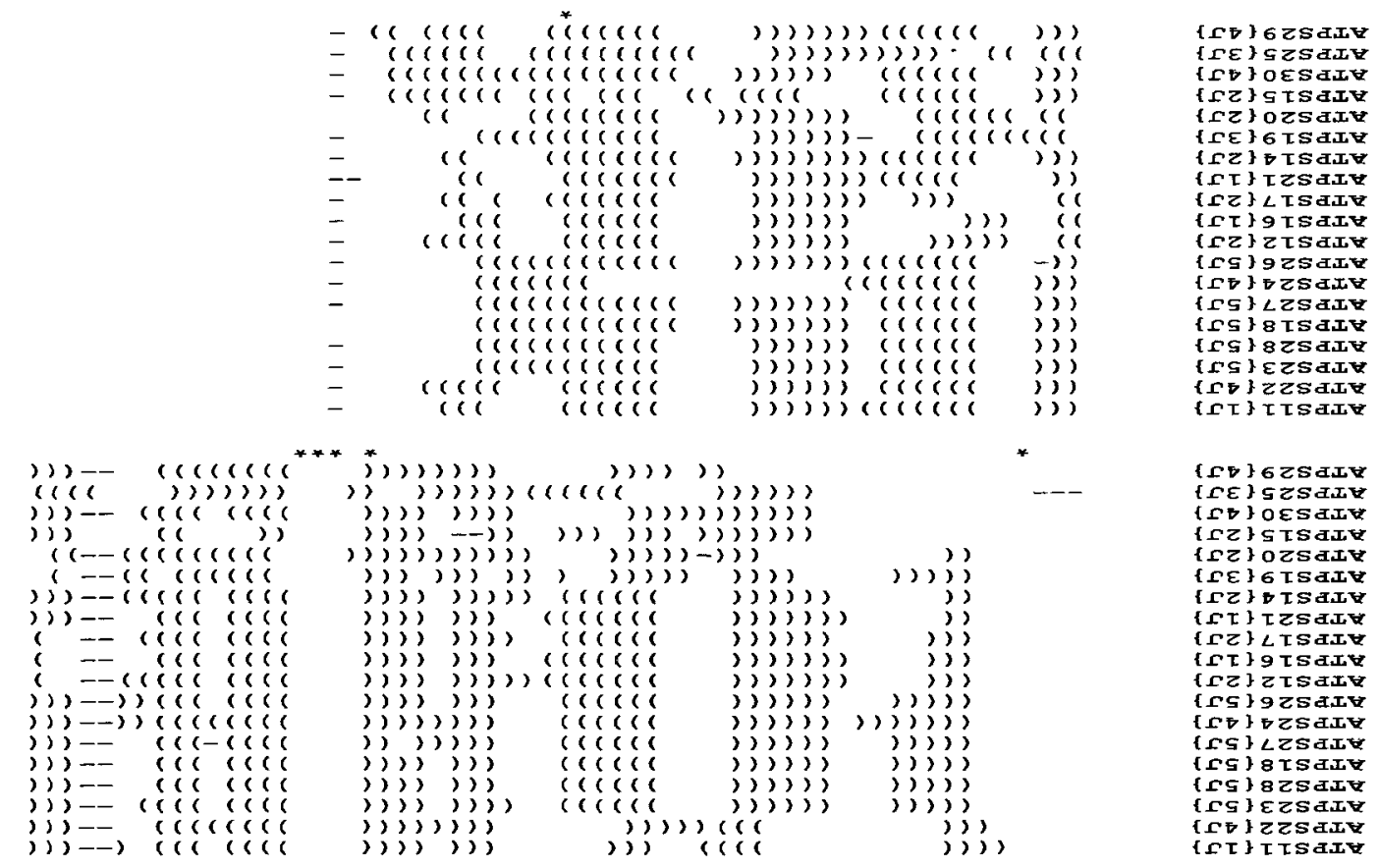


secondary structures. RNA/DNA secondary structure prediction through free energy minimization, which is also called RNAfold, is the most widely used function in ViennaRNA. Structure prediction by ViennaRNA generates a single optimal structure based on a dynamic programming algorithm originally developed by Zuker and Stiegler [192]. This method predicts secondary structures based on minimal overall free energy derived from stacked pair and loops [65]. In this respect, stacks are formed by two consecutive base pairs, and loops include internal loops, bulges, hairpin loops and multiloops (junctions). The algorithm explores all the possibilities when computing the minimum free energy structure recursively by dynamic programming.

Mfold is another RNA/DNA folding package using thermodynamic methods [116, 191]. Hofacker and coworkers compared the performance of RNAfold and Mfold, and found that RNAfold is considerably faster than Mfold [65]. Recently, other folding programs such as GTfold [117] and MCfold [133] have been developed. Figure 2.25 shows the comparison of structure distributions of the same 1000 random sequences predicted by RNAfold, GTfold and MCfold. The structure distributions generated by RNAfold and GTfold are almost identical (see Table 2.7). However, MCfold predicted considerably more complex structures. Upon closer inspection of MCfold, we observe that this method starts by enumerating all possible junction configurations and therefore has a tendency to overestimate the number of complex structures. We also observed a considerable difference in runtime between these three methods. On a single machine, the run time required for a single sequence by RNAfold and GTfold are very similar [117]. However, MCfold runs several orders of magnitude slower. On a PC using an Intel Core 2 Quad Q6600 processor with 2.4GHz and 8GB DDR2 RAM, the run time of RNAfold for folding one sequence was about 0.008029 seconds, while it took about 845 seconds for MCfold, which is approx. 100,000 times slower than RNAfold. Thus, it would take 27 years for MCfold to predict the secondary structure for one million sequences. It is therefore not feasible to use MCfold to predict secondary structures in our methods despite its capacity in predicting pseudoknot structures.

Currently, both our approaches for pool design are based on RNA/DNA secondary 


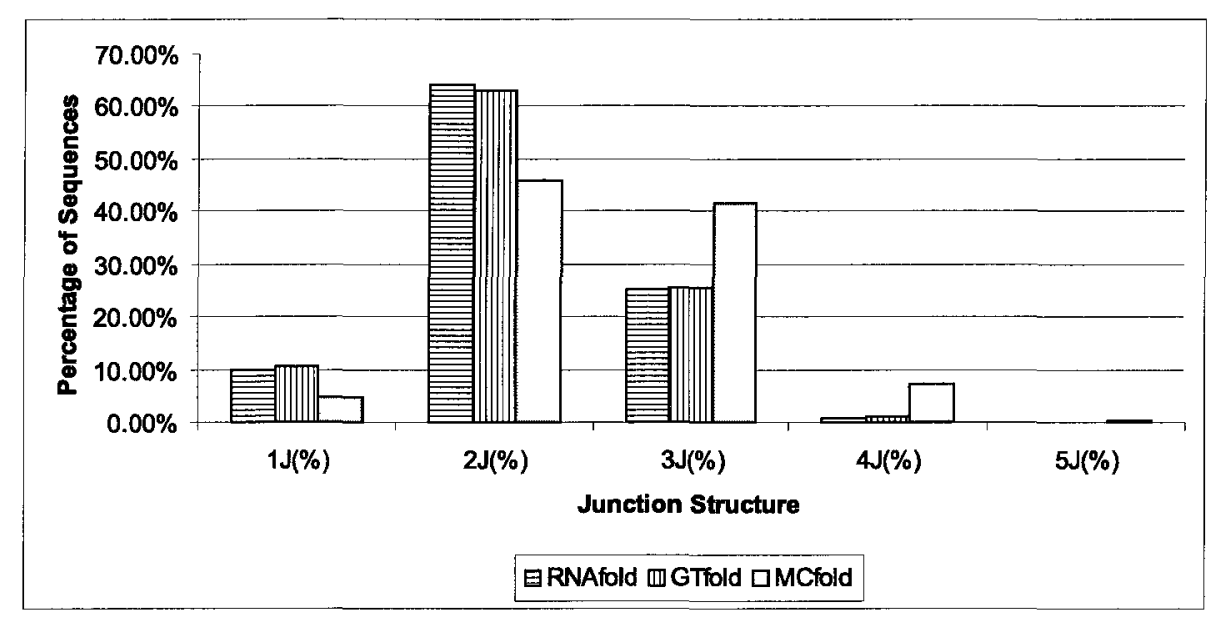

Figure 2.25: Comparison of predicted structural distributions of RNAfold, GTfold and MCfold for the same 1000 random sequences of 100 nucleotides each (containing no fixed primer sites). The structure distributions generated by RNAfold and GTfold are almost identical. However, MCfold predicted considerably more complex structures.

structure folding algorithms, such as RNAfold in the ViennaRNA package. Consequently, our approaches are subjected to the limitations of these folding algorithms. As an example, RNA sequences that adopt pseudoknot structures are not amenable by our approaches since RNAfold in ViennaRNA package cannot fold a sequence into a pseudoknot. This is a concern because functional RNA pseudoknots have been found in random pools [168]. Although there exist algorithms that can fold pseudoknot structures, such as PKNOTS [143], MCfold [133] and gfold [142], folding of large sets of sequences as required in our methods is not efficient and feasible. However, this thesis is concerned with small RNAs (100 nt) only and focuses on statistical properties such as frequencies of junction structures for large numbers of sequences, thus impact of the lack of ability to predict pseudoknots is minimized.

\begin{tabular}{|c|c|c|c|c|c|}
\hline & 1J Structures & 2J Structures & 3J Structures & 4J Structures & 5J Structures \\
\hline RNAfold & $19.65 \%$ & $20.39 \%$ & $18.63 \%$ & $20.48 \%$ & $20.86 \%$ \\
\hline GTfold & $19.96 \%$ & $20.01 \%$ & $18.55 \%$ & $21.25 \%$ & $20.23 \%$ \\
\hline
\end{tabular}

Table 2.7: Structural distribution comparison between RNAfold and GTfold for GFPool1. GFPool1 is shown in Figure 2.17. 


\subsubsection{Comparison of Inverse Folding with Random Filtering}

Inverse Folding associated with the ViennaRNA package was developed to find sequences that fold into a predefined structure [64]. However, it cannot be applied for the design of starting pools for in vitro selection experiments because of the primers involved in the experiments. Inverse Folding cannot generate sequences containing primers directly. As shown in Figure 2.26, adding primers to complex structures can have a dramatic effect. In the experiment shown, we consider 20 million $5 \mathrm{Js}$ of length 70nt generated by Inverse Folding and add to each of them two primers of length 15nt. As shown in Figure 2.26, all except 155,989 structures change from 5Js to simpler structures. That is, less than $1 \%$ of engineered sequences retain $5 \mathrm{Js}$ and instead result in simpler structures.

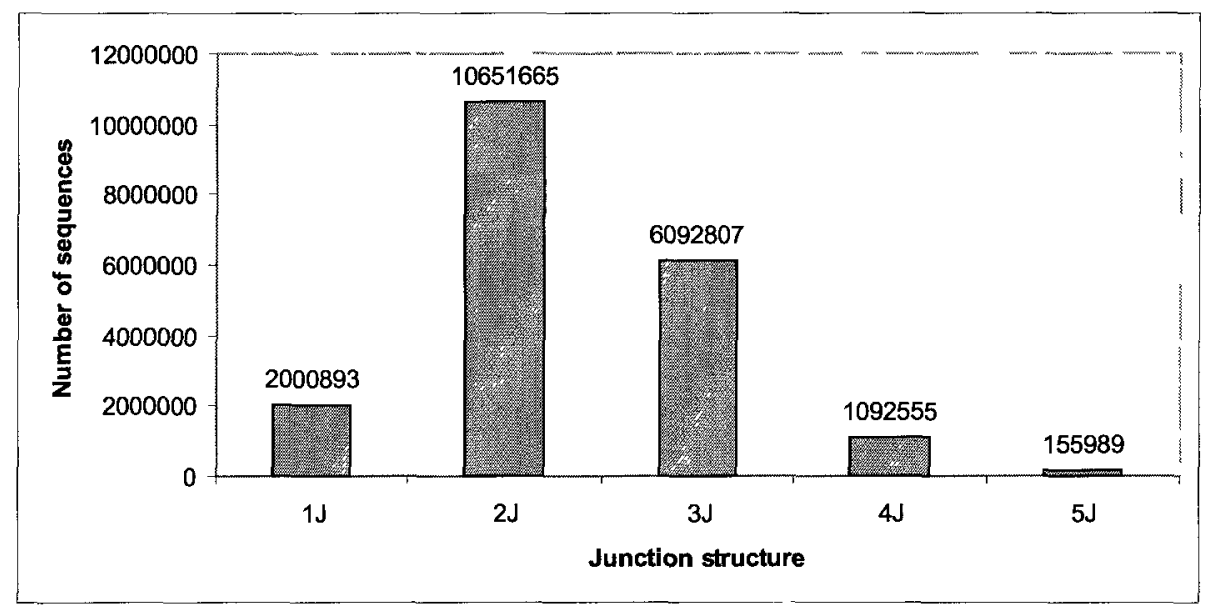

Figure 2.26: Resulting structural distribution after addition of two $15 \mathrm{nt}$ primers to 20 million 5-way junctions sequences of length 70 nt generated by Inverse Folding. Less than $1 \%$ of sequences retain 5-way junctions and instead result in simpler structures.

To compare the Inverse Folding method and the Random Filtering method, we randomly chose $465 \mathrm{~J}$ structures generated by Inverse Folding in Figure 2.26 and a random pool, respectively, and mutated their single stranded positions 1 million times. Random Filtering generates more complex structures than Inverse Folding (Figure 2.27) and would be more suitable to generate structurally diverse starting pools for in vitro selection experiments. 


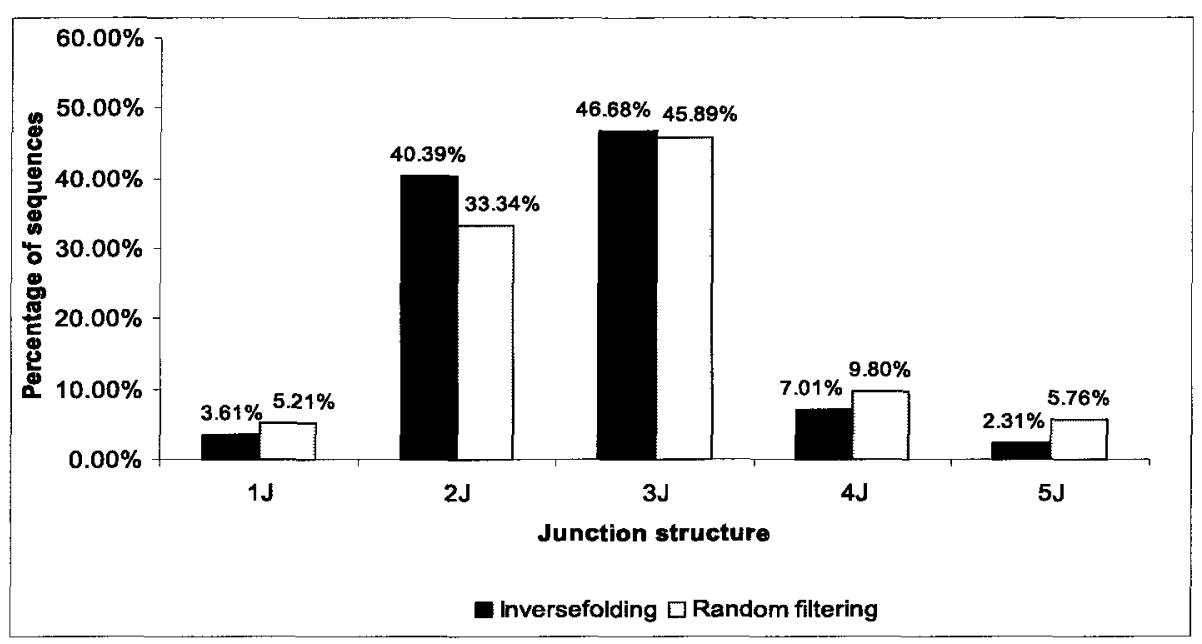

Figure 2.27: Comparison of average structural distribution for the pools generated by Random Filtering and Inverse Folding. Random Filtering generates more complex structures than Inverse Folding.

\subsubsection{Comparison of Genetic Filtering with the Mixing Matrices ap- proach}

Schlick and coworkers $[86,88]$ developed a computational approach for designing structured RNA pools by modeling the pool generation process using mixing matrices. To compare our method with the mixing matrices approach, we developed a program called RNA To Tree to convert RNA/DNA secondary structures into the tree structures as defined in $[52,53]$. To design a pool with a uniform distribution of 12 tree structures from $2_{1}$ to $6_{5}$ in [53], we used Genetic Filtering to create a single design that would yield a startup pool with a uniform distribution of 12 tree structures (see Section 2.4.3).

Applying the mixing matrix approach to create a pool with a uniform distribution of 12 tree structures (from $2_{1}$ to $6_{5}$ ) resulted in error rates of $60 \%$ (using 1 mixing matrix) and $38.9 \%$ (using 2 mixing matrices), respectively. Here, errors refer to the sum of the distances of individual to the desired distribution, i.e. $8.33 \%$ for uniform distribution of 12 tree structures. In contrast, Genetic Filtering reduced the error to $35.23 \%$ (with one single pool which is equivalent to 1 mixing matrix). For in vitro experiments, our method requires only one synthesis, whereas the mixing matrices approach needs one synthesis for each mixing matrix. Therefore, our method is also 
less costly and less time consuming compared with the mixing matrices approach.

Our results complement the mixing matrices approach in several ways. Random Filtering and Genetic Filtering are able to add more complex structures to the pool. For example, the most complex structures created via mixing matrices are $4 \mathrm{~J}$ structures whereas Random Filtering and Genetic Filtering can increase and control, respectively, the number of $5 \mathrm{~J}$ structures in the pool. The mixing matrices approach also does not cover the inclusion of primers. However, primer-binding regions are required in essentially all in vitro selection experiments. Since the primer regions are considered in our approach, our designed pools can be directly implemented into in vitro selection experiments. While we showed how Genetic Filtering generates pools with uniform structure distributions, the method can be used to design any given target structure distribution, not only for junction structures, but also for tree structures. The approach can also be applied to design pools including specific substructures such as common structural components of aptamers.

\subsection{Conclusions and future work}

In this study, we developed two new systematic computational pool design approaches that increase the covered search space for in vitro selection of complex aptamers: Random Filtering and Genetic Filtering. Compared with close to zero five-way junctions and around $0.2 \%$ four-way junctions in a typical random DNA pool with $10^{6}$ structures of 100 nucleotides each, Random Filtering generated pools of the same size where more than $10 \%$ of the sequences are five-way junctions and more than $15 \%$ of the sequences are four-way junctions. Very similar results were found for 100nt DNA pools where 30nt are fixed primers. Our RFPool A (designed via Random Filtering) was synthesized and a few sequences were determined and folded using ViennaRNA. In a subset of only 17 sequences, $11.76 \%$ were found to be 5 -way and $11.76 \%$ were 4 -way junctions. Compared to a completely random pool, our Random Filtering method can dramatically increase the likelihood of obtaining high-order junctions experimentally.

Our second method, Genetic Filtering can generate DNA pool designs of 100 nucleotides each, where 30nt are fixed primers, with distributions in silico that are very close to a uniform structure distribution, i.e. highly diverse. Analysis of a small 
set of the sequences obtained from the GFPool1 (designed via Genetic Filtering) in vitro showed significant structural diversity, with each of the $1 \mathrm{~J}, 2 \mathrm{~J}, 3 \mathrm{~J}, 4 \mathrm{~J}$, and $5 \mathrm{~J}$ structures present at levels of at least $10 \%$. Complex $5 \mathrm{~J}$ structures accounted for $26 \%$ of the sequences prior to enrichment. After GFPooll was subjected to 8 rounds of selection against the target ATP, complex $5 \mathrm{Js}$ accounted for $20 \%$ of the sampled sequences. Given that in a fully random pool only 35 of 20 million sequences result in complex $5 \mathrm{~J}$ structures, the likelihood of observing this concentration of $5 \mathrm{~J}$ structures is approximately $10^{-10}$. These results confirm that our suggested approach not only leads to better access to more complex structures for in vitro selection experiments but also that SELEX is capable of preserving highly complex structures if they are present in the initial DNA pool. When the binding affinities of two $5 \mathrm{~J}$ sequences from this enriched pool were tested, one sequence (ATPF8) displayed stronger affinity for ATP than Huizenga and Szostak's low complexity aptamer, while the other (ATPF4) showed weaker affinity. This suggests that higher complexity sequences have the potential to be better aptamers, but will not always lead to improved binding. Thus, structural diversity, not just structural complexity, is an important characteristic of an ideal starting pool for SELEX. Our Genetic Filtering method is particularly aimed at designing such pools. Future work will examine in more depth whether these designed pools lead more generally to aptamers with improved binding characteristics. 


\section{Chapter 3}

\section{Identification of transposon insertion polymorphisms by computational comparative analysis of next generation personal genome data}

This chapter focuses on the development and implementation of computational methods to identify transposon insertion polymorphisms by analyzing next generation personal genome data. A paper submitted to the American Institute of Physics Proceedings of AMMCS-2011 [110] is partly the basis for this chapter. I carried out all computational work for this project. The experimental validation was performed by Irene Lozynska in Dr. Liang's laboratory at Brock University.

\subsection{Introduction}

Structural variations (SVs) in a genome is commonly defined as DNA sequence alternation among individuals of the same species that are not single nucleotide polymorphisms (SNPs) [43, 172]. Such variants include deletion, duplication, insertion, inversion, and translocations of DNA sequences [50]. Rapidly accumulating evidence indicates that SVs significantly contribute to human diversity and disease susceptibility. Many SVs are associated with genetic diseases such as psoriasis, autism and Crohn's disease $[66,115,120]$. Therefore, detecting and characterizing these SVs is an important step in understanding the genetic basis of these diseases. However, identification and characterization of SVs have proven to be a very challenging task [41]. In recent years, with the development of new sequencing technologies, such as Roche 454, Illumina Solexa, and ABI SOLiD, a vast amount of high throughput sequencing (HTS) personal genome data has been generated. One example of such effort is the 1000 genome project that has so far generated deep or low coverage sequence data for more than 1000 individuals covering major human populations [81, 42]. These 
personal genome data that are expected to increase rapidly in the next few years will open new opportunities for comprehensive analysis of SVs. Recently, a number of computational tools have been developed for the identification of SVs using these personal genome data [123]. However, SVs identified by these methods do not include transposon insertions, a type of SV that is more challenging to identify due to the highly repetitive nature of the transposon sequences, i.e. huge number of copies in the genome and very similar sequences among the same family members.

Transposons or transposable elements (TEs) are discrete pieces of DNA that can move within a genome. TEs, with several millions of copies classified into many families and subfamilies, account for approximately $45 \%$ of the human genome [34], and they play important roles in the evolution of the genome and regulating gene functions $[6,79,34,59]$. By simply moving from one place to another or by making new copies inserting into other locations in the genome, these TEs generate a major source for inter- and intra-species genetic diversity [184, 21, 60, 155]. Transposon insertion polymorphism (TIP) refers to the presence or absence of a transposon insertion at a specific genomic location in populations of a given species. A small fraction of the TEs remain active today, and recent evidence indicates that about 35 to 40 subfamilies of Alu, L1, SVA elements and possibly HERV-K elements remain actively mobile in the human genome [126]. As discussed previously in Introduction, variations in DNA sequence may be either common (more than 1\%) or rare in a population. Polymorphisms refer to the ones in the former case and private polymorphisms or mutations refer to those in the latter case [13]. In this thesis, all types of transposon insertion variations are referred to as transposon insertion polymorphisms. Many dozens of disease-causing transposon insertions have been identified [26, 12, 132], and the full scope of transposon mutagenesis in humans is likely to extend far beyond these initial cases.

Due to the presence of TEs in an extreme large number of copies and the highly similar sequences among members in the same family, genome-wide analysis of TIPS presents a great deal of challenges. Only a small number of TIPs have been identified using classical molecular biology techniques, such as locus-specific PCR, targeted mutation screening, and transposon differential display polymerase chain reactions 
(PCR) $[185,5,11,146]$. With the completion of the human genome project and availability of limited additional personal genome data, a few studies have explored the use of computational comparative genomic approaches to identify TIPs and these approaches have proven to be very fruitful $[177,96,179,185]$. These early studies not only demonstrated the usefulness of personal genome data in identifying TIPs, but also suggested to us that the level of human genetic diversity associated with TIPs is much higher than what was previously appreciated. The latter point has been further confirmed by several recent pilot studies employing experimental analysis of personal genomes using next generation sequencing technologies $[183,47,71]$. With the availability of personal genome data increasing at a unprecedented scale, it has become imperative to develop new computational algorithms in order to utilize these genomic data for the study of TIPs.

In this study, we developed a computational method, called TIP-finder, to identify TIPs through analysis of next generation personal genome data. In TIP-finder, a greedy algorithm is used to identify the candidate TIPs loci which are then enhanced by a machine-learning approach. We tested the efficiency of TIP-finder with simulated data and were able to detect about $88 \%$ of TIPs with a precision of $\geq 91 \%$. When TIP-finder was used to analyze the Solexa paired-end sequence data at deep coverage for six genomes representing two trio families, a total of 5569 TIPs, consisting of 4881, 456, 91, and 141 insertions from Alu, L1, SVA and HERV, respectively, were identified, representing the most comprehensive analysis of such type of genetic variation so far.

\subsection{Literature review}

\subsubsection{Genome}

The genome refers to all DNA (in some viruses, RNA) sequences in an organism[134]. It is an entirety of the genetic material of an organism passed along from one generation to the next generation. Genomic DNA is an unbranched polymer in which four distinct nucleotides are linked together in a given order. The variability of the four bases constitutes the genetic code of DNA. The particular order of $A, G, C$ and $T$ is extremely important and it contains all necessary information for living organisms to 
survive and reproduce. Therefore, determining the DNA sequence is very useful in fundamental research in understanding why and how organisms live.

The size of genomes varies from one species to another, from a few thousand base pairs to a few billions base pairs, from thousand genes to tens of thousand genes. For example, the phi $X$ bacteriophage, the first sequenced DNA-based genome, has about 5 thousands base pairs [148], while the human genome has about 3 billion base pairs. The E. coli genome contains about 4000 genes and the human genome contains about 23000 genes. Genes account for only about 1-2 percent of the human genome, and the remaining part of the genome consists of regulatory sequences, transposons and sequences with unknown functions[76].

Genomes are organized into physical structural unites called chromosomes. In the case of humans, the genome is composed of 23 pairs or a total of 46 chromosomes. The shortest chromosome is 50 million base pairs and the longest is 260 million base pairs [19]. Every child receives two sets of 23 chromosomes: one from the mother and one from the father. Of these 23 pairs, 22 pairs are called autosomes, and they are the same for both males and females. The 23rd pair, the sex chromosomes, differs between males and females. Females have two copies of the $X$ chromosome, and males have one $X$ and one $Y$ chromosome. The two chromosomes in a pair come from each parent, so they are not identical in sequence due to the high level of genetic diversity.

\subsubsection{Genome sequencing}

DNA sequencing is defined as the process and methods for determining the order of the nucleotide bases $(A, G, C$ and $T$ ) in a DNA molecule. It provides the information of the sequence of nucleotides. Over the past few decades, a few sequencing methods have been established. Very recently, next generation sequencing methods were developed to meet the increasing demand in genome sequencing. As of September 2009, 5843 genome sequencing projects have been recorded, of which 1100 have been completed, including 982 prokaryote species and 118 eukaryotic species [109]. 


\subsubsection{Sanger sequencing}

In 1977, two DNA sequencing methods were independently developed, one by an American group led by Maxam and Gilbert [118] and the other by an English group led by Sanger [150]. The Maxam-Gilbert method was based on a chemical cleavage protocol, and its reaction products were separated by gel electrophoresis. The Sanger group designed a procedure similar to the natural DNA replication process. It is based on the use of 2',3'-dideoxynucleoside triphosphates (ddNTPs) as DNA chain terminators.

Both groups shared the Nobel prize in 1980, however, the Maxam-Gilbert sequencing rapidly became out of favor due to the extensive use of hazardous chemicals and difficulties in automation. While the Sanger method has become the dominant approach and gold standard for DNA sequencing in recent decades [151], it has been modified to improve its efficiency, which includes the use of capillary electrophoresisbased sequencing machines for automated detection of DNA molecules with improved thermostable polymerases and fluorescent dye-labelled dideoxynucleotide terminators [134]. The Sanger method was used in the sequencing of the first human genome, which was initially published in 2001 [75], further improved in 2004 [76] through the Human Genome Project. The current Sanger sequencing can achieve read lengths of $800-1000$ bp with a per-base "raw" accuracy as high as 99.999\% [162].

For the past 30 years, continued improvements in Sanger sequencing techniques, associated with advances in computing and informatics, have reduced the cost of genome sequencing by about two orders of magnitude and have shortened the genome projects from a decade to mere months (for mammalian-sized genomes), or even weeks (for microbial genomes)[145]. However, it still costs at least $\$ 10$ million to sequence a human genome or other genomes at a similar size [129]. Only a small number of large genome centres worldwide have the resource to handle the sequencing of a mammalian-sized genome. With the promise of individualized medicine (pharmacogenomics), new sequencing methods are being sought to decrease the cost and time required for genome sequencing. 


\subsubsection{Next-generation sequencing (NGS)}

NGS refers to platforms for DNA sequencing in a massively parallel manner in replacing the Sanger method. In 2005, a new platform using pyrosequencing technology was developed by 454 Life Sciences (http://www.454.com) [114] and this was the first commercial NGS platform. The method shears DNA into fragements of a few hundred bp and then amplifies DNA inside water droplets in an oil solution (emulsion PCR), with each droplet containing a single DNA template attached to a single primercoated bead that then forms a clonal colony. The sequencing slide contains many picolitre-sized wells each containing a single bead and sequencing enzymes. Pyrosequencing uses luciferin to generate light for detection of the individual nucleotides added to the existing DNA, and then determine the sequence of the growing strand [114]. When the 454 platform was first introduced, it was able to sequence 25 million bases in a four-hour period, about 100 times faster than the current Sanger sequencing [114]. The current 454 instrument, the GS-FLX, produces an average read length of $400 \sim 600 \mathrm{bp}$ per read, with a combined throughput of $1 \mathrm{~Gb}$ of sequence data per 10-h run (see Table 3.1) [175]. Its cost was reported as $\$ 60$ per Mb in 2008 [162].

In 2006, the Illumina (Solexa) genome analyzer, the first short-read sequencing platform, was commercially launched by Solexa, now part of Illumina (http://www.illumina.com This sequencing technology is based on reversible dye terminators. DNA molecules are first attached to primers on a slide and amplified so that clonally amplified clusters are generated by bridge amplification. Four differently labeled ddNTPs are added and non-incorporated nucleotides are washed away. Unlike pyrosequencing, the DNA can only be extended one nucleotide at a time. The fluorescently labeled nucleotides are recorded and the dye is chemically removed from the DNA before the next synthesis cycle. The current Solexa instrument produces an average read length of $100 \mathrm{bp}$ per read, with a combined throughput of $50 \mathrm{~Gb}$ of sequence data per 9-day run (see Table 3.1) [175]. Its cost was reported as $\sim \$ 2$ per $\mathrm{Mb}$ in 2008 [162].

The SOLiD (Supported Oligonucleotide Ligation and Detection) sequencing platform, introduced by Applied Biosystems (http://www.solid.appliedbiosystems.com) in 2007, is a short-read sequencing technology based on ligation. A pool of all possible oligonucleotides of a fixed length are labeled according to the first two bases. 
Oligonucleotides are annealed and ligated; the preferential ligation by DNA ligase for matching sequences results in a signal informative of the nucleotides at that position. Before sequencing, the DNA is amplified by emulsion PCR. The resulting beads, each containing only copies of the same DNA molecule, are deposited on a glass slide [173]. The current SOLiD instrument produces an average read length of 50bp per read, with a combined throughput of $60 \mathrm{~Gb}$ of sequence data per 5-day run (see Table 3.1) [175]. Its cost was reported as $\sim \$ 2$ per $\mathrm{Mb}$ in 2008 [162].

Roche (454), Illumina (Solexa) and ABI (SOLiD) are the main NGS platforms on the current market. Although they are based on different approaches, they share common technological features: All these platforms run sequencing in parallel to increase the throughput and they are capable of generating paired-end data that allow for paired-end mapping (PEM, see detailed definition in Section 3.3.1) techniques to be used for the detection of structural variations.

NGS technologies have reduced sequencing cost from $\$ 0.01 /$ base in 2004 to nearly $\$ 0.0001 /$ base in 2006 and increased the sequencing capacity from $1 \mathrm{Mbp} / \mathrm{machine} /$ day in 2004 to more than $5 \mathrm{Gbp} /$ machine/day in 2009 [169]. Compared with the Sanger sequencing method, NGS technologies are orders of magnitude faster and cheaper. In the short 5 years since the first commercial platform became available, NGS has been accelerating and revolutionizing a wide variety of genomics research, enabling experiments previously not technically feasible or affordable to be performed. Furthermore, these technological advancements opened entirely new areas of biological inquiry, such as the investigation of ancient genomes, the characterization of ecological diversity, and the identification of unknown etiologic agents [113]. However, compared with Sanger sequencing technique, NGS technologies are limited by the short read lengths and the accuracy of base calls, which makes the assembly of the whole genome from raw read data much more difficult.

\begin{tabular}{lrrr}
\hline Technology & Read Length & Output(GB) & Run Time \\
\hline Roche/454 & $400-600$ & 1 & $10 \mathrm{~h}$ \\
Illumina/Solexa & 100 & 50 & 9 days \\
ABI-SOLiD & 50 & 60 & 5 days \\
\hline
\end{tabular}

Table 3.1: Summary of the capacity for the major next-generation sequencing technologies [175]. 


\subsubsection{Genome sequencing strategies}

Since the available methods for DNA sequencing can only handle short stretches of DNA (less than 1000 base pairs) at a time, to sequence a longer DNA such as a genome, special strategies are required. In 1977, shotgun sequencing was introduced by Sanger et al $[150,166]$. In this method, the genome of an organism is randomly cut into small fragments by enzymes, each of which is then cloned and sequenced separately. The sequence is a "read" by a sequencing instrument, resulting in a collection of reads. Reads are the sequences of individual DNA fragments from a single run [136]. Normally, the length of the DNA fragments is longer than the length of reads, so only the ends of DNA fragments are sequenced. The reads are then combined in a proper order to obtain the sequence of the whole genome using computer programs called genome assemblers. In order to assemble the original genome sequence, each base in the genome needs to be sampled by at least one of the reads. The amount of DNA that is sequenced is referred to as coverage, which is defined as the ratio between the cumulative size of the reads and the size of the genome, i.e. : $c=L \times N / G$, where $L$ is the read length, $N$ is the number of reads, and $G$ is the length of the genome.

Currently, two shotgun strategies are used for genome sequencing. One strategy is called hierarchical shotgun sequencing, also referred to as "map-based", "BACbased" or "clone-by-clone" (see Figure 3.1). In this method, the DNA is first cut into fragments by enzymes and each fragment is inserted into a bacterial artificial chromosome (BAC). A BAC is a genetically engineered DNA that can replicate inside a bacterial cell. Each BAC clone can contain an insertion of foreign DNA from 100 to $500 \mathrm{~kb}$. The collection of BACs containing the whole genome is called a BAC library. The BAC inserts are isolated and mapped onto chromosomes using methods such as FISH to determine the order of each cloned fragment in the chromosome. Each BAC fragment is then randomly fragmented into smaller pieces (50 kb for cosmid libraries and 2 to $10 \mathrm{~kb}$ for plasmid libraries) and each piece is sequenced from both ends. The sequences are aligned so that the identical sequences overlap, and then these "shotgun" sequences are assembled into contigs (contiguous sequences) [134].

The other genome sequencing strategy is called whole genome shotgun (WGS) sequencing (see Figure 3.2). This method was first applied by Sanger and colleagues 


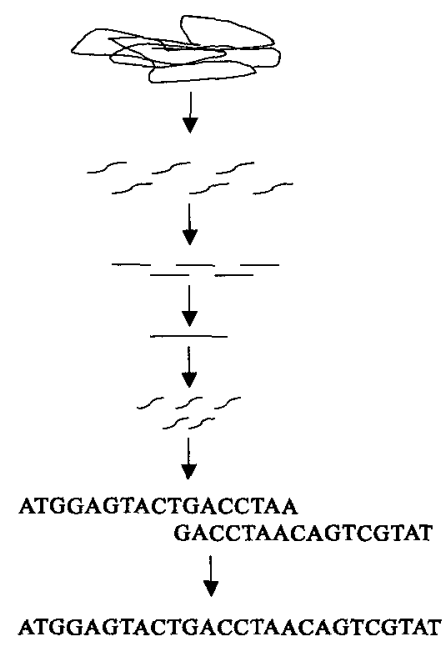

genomic DNA

BAC library

Mapped BAC clones

BAC to be sequenced

shotgun clones

shotgun sequence

assembly

Figure 3.1: Hierachical shotgun sequencing method [134].

to the genome of bacteriophage $\chi$ [149]. WGS randomly shears genomic DNA into fragments and then the fragments are sub-cloned into small-insert libraries such as 2 $\mathrm{kb}$, and large-insert libraries such as 10 to $20 \mathrm{~kb}$. The fragments are then sequenced from both ends. Once the sequences are obtained, they are aligned and assembled into contigs on the basis of sequence overlap [134].
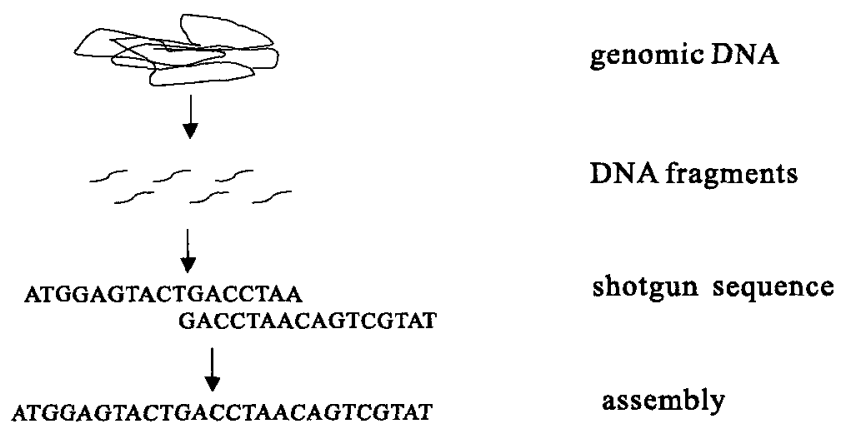

Figure 3.2: Whole genome shotgun sequencing method.

Each of these approaches has advantages and disadvantages. Unlike WGS sequencing, the location for each BAC is known in the hierarchical shotgun sequencing, so its sequence assembly is focused on a small region of the genome. Thus, there are 
fewer random fragments to assemble and the sequencers are more reliable. The disadvantage of the hierarchical shotgun sequencing is time and expense. The BAC clone mapping step can be especially time consuming. By contrast, the WGS sequencing is very fast and less expensive, but it is very computationally challenging to put a large number of short fragments back into a full sequence. It also imposes a greater potential for large-scale misassembly. Both approaches have been used to sequence whole genomes. The hierarchical shotgun sequencing method has been used for many large, eukaryotic genomes, including the genome of S. cerevisiae and the public consortium's version of the Human Genome Project [75]. The WGS method was used to sequence the genome of the bacterium Haemophilus influenzae [51], and it was also employed by the privately funded company Celera Genomics to sequence the human genome. The WGS method is currently used by all next-generation sequencing technologies.

\subsubsection{The human genome projects}

The Human Genome Project (HGP) was first launched in the US in 1990 as a joint effort of the Department of Energy and the National Institutes of Health. During the early years. of the HGP, the International Human Genome Sequencing Consortium (IHGSC), an open collaboration including twenty centres in six countries, was formed to carry out the component of the HGP [75]. In 1998, a private biotechnology company, Celera Genomics, started a project in sequencing human genome. In February 2001, both the IHGSC [75] and Celera Genomics [174] reported the draft sequences of the human genome. Improved drafts were later announced in 2004 [76]. The genome sequences generated by the public HGP and private project are called the human genome reference sequences. The reference sequences do not represent any specific person's genome, and are actually a consensus sequence for a few different genomes.

Personal genome projects aimed to sequence individual human genomes. In 2007, Levy and colleagues [105] published the first complete personal genome - Craig Venter's DNA sequence. In the following year, Wheeler and colleagues [180] also reported the complete genome sequence of James D. Watson. This is the first genome sequenced by next-generation sequencing technologies.

In January 2008, the 1000 Genomes Project (http://www.1000genomes.org/) was 
announced to sequence the genomes of at least 1000 people from around the world, using the NGS technologies. It aims to provide the most detailed and medically useful resource on human genetic variation. The goal of the 1000 Genomes Project is to find most of the variants in the human genome that have a frequency of at least $1 \%$ in the populations studied. The 1000 Genomes Project has completed its pilot phase, designed to develop and compare different strategies for genome-wide sequencing with high-throughput platforms [42].

\subsubsection{Structural variation in the human genome}

Human genetic variations can be categorized into various size ranges. The smallest type of variation is at the single nucleotide polymorphism (SNP) level, and it affects only one single nucleotide base. The international HapMap project [74, 73] documented 3.1 million SNPs from 270 human individuals. However, human genetic variation extends beyond SNP. SVs can be defined as polymorphic rearrangements of genome of $50 \mathrm{bp}$ up to hundreds of $\mathrm{kb}$ in size [160]. Recent studies have defined approximately $5 \%$ of the human genome as structural variant in the normal population, involving 800 independent genes [160].

Structural variations in the human genome include deletion, duplication, insertion, inversion, translocation and transposon insertion. All these variants are relative to the human reference genome. Deletion/insertion occurs when there is a less/more segment of DNA relative to the reference genome. For example, if the human chromosome has sections in order of $\mathrm{A}-\mathrm{B}-\mathrm{C}$ in the reference genome, then deletion/insertion occurs when a genome has sections $\mathrm{A}-\mathrm{C}$ (deletion) or $\mathrm{A}-\mathrm{E}-\mathrm{B}-\mathrm{C}$ (insertion). Inversion occurs when a segment of DNA that is reversed in orientation with respect to the rest of the chromosome such as having sections A-C-B. Tandem duplication occurs when two identical segments are adjacent in the chromosome, i.e. A-A-B-C. Translocation is defined as a change in position of a chromosomal segment within a genome that involves no change to the total DNA content. Translocation can be intra- or interchromosomal [50].

Rapidly accumulating evidence indicates that SVs contribute significantly to human phenotype diversity with many SVs associated with genetic diseases such as 
psoriasis, autism and Crohn's disease [66, 115, 120]. Therefore, detecting and characterizing these SVs is an important step in understanding the genetic basis of these diseases.

\subsubsection{Methods for structural variation detection}

After the completion of the human genome project, the reference genome has been playing an important role in the detection of SVs. It is now possible to use computational comparative genomic approaches described below to identify SVs through a comparison to the reference genome.

\subsubsection{Array-based methods}

The earliest methods for detecting SVs used array comparative genome hybridization (aCGH), which tests the relative frequencies of probed DNA segments between two genomes [135, 154, 161]. Another approaches use single-nucleotides polymorphism (SNP) arrays (available from the HapMap project) to measure the intensity of probe signals at known SNP loci. By considering allelic ratios at heterozygous sites, these methods based on arrays are able to detect copy-number variants(CNVs) such as insertion and deletion $[120,33,31,62,119]$. However, the size and breakpoint resolution of any prediction depend on the density of the probes on the array, which is limited by either the density of the array itself (for aCGH) or by the density of known SNP loci (for SNP arrays) [123]. Further, these platforms are unable to detect balanced structural variations such as inversion and translocation, and they have to avoid the repetitive part of the genome. Thus, they are unable to detect transposon insertions.

\subsubsection{Paired-end mapping (PEM)-based methods}

Detecting SVs between two individuals would be an easy task if both of the genomes were already assembled. However, until now only few human genomes have been assembled, i.e. the human genome reference sequences, Venter's and Watson's personal genomes. Therefore, current computational methods to detect structural variations use only one assembled genome (the "reference") and another sequenced but unassembled genome (the "test"). 
Paired-end mapping (PEM), enabled by most of the NGS technologies, has been successfully implemented to detect SVs by comparing a test genome with the reference genome [123]. PEM involves the generation of paired-end reads, which represent the two short reads of the two ends of a genomic fragment with a known estimated size, i.e. the library size or the insert size. The paired-end reads are sampled from the test genome, where they are ordered with certain orientations such as head to head $(\rightarrow \leftarrow)$ orientations. Although the test genome cannot be compared with the reference genome directly, when the paired-end reads are mapped to the reference genome, SVs can be discovered based on the patterns of PEM characteristic of each type of SVs. PEM behaves differently in the region surrounding the SVs depending on the nature of SVs [123]. Basically, alignments of paired-end reads to the reference genome are categorized as concordant and discordant [172]. Paired reads mapped to the reference genome at a distance similar to the inserted size and in a correct orientation represent a concordant PEM, otherwise represent a discordant PEM, which indicates a potential SV.

Figure 3.3 shows the signatures patterns of PEMs that are characteristics of different structural variations $[123,67]$. Insertions and deletions can be detected when the mapped distance is different from the inserted size. If the mapped distance is smaller than the inserted size, it is an insertion, otherwise a deletion. As for inversion, the mapping order of the two reads does not change but the orientation of one of the reads changes. Tandem duplication occurs when the paired-end reads mapped to the reference out of order but with proper orientations. When the paired-end reads are mapped confidently on different chromosomes, a translocation event is indicated.

Different types of SVs have different types of PEM signatures, so most current PEM-based methods detect SVs through the PEM signatures, which are dependent on mapped distance, order and orientations. Incorrect mapping information can lead to errors in the detection of PEM signatures. In order to reduce the chances of false positive, current methods group signatures that support the same variation together into a cluster. A standard clustering strategy, introduced by Tuzun and co-workers [172], only considers the discordant PEMs and ignores any paired read that has more than one good mapping position. Paired reads are considered as discordant when their 

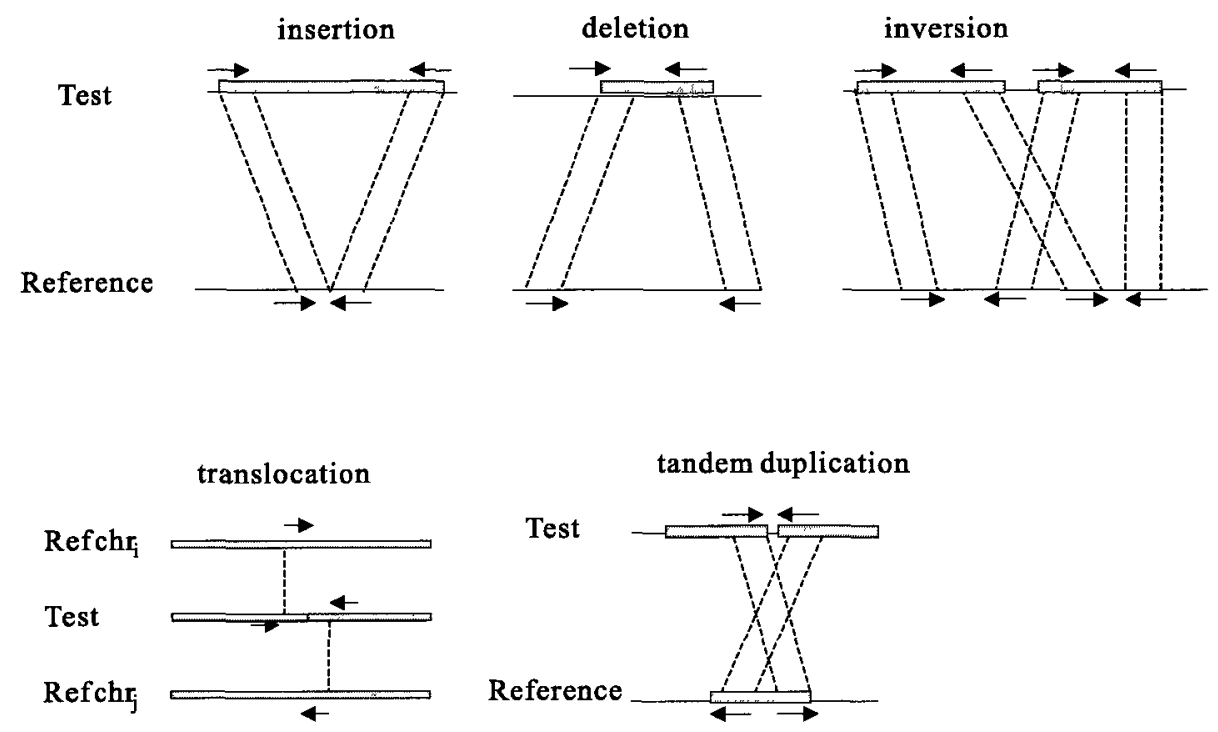

Figure 3.3: Types of structural variation that can be detected with PEM [123, 67].

mapping distance is 2-4 standard deviations away from the mean insert size. A cluster is formed if there is at least two or other minimum number of pairs showing signatures of the same type and with similar size and location. This standard clustering strategy was later used in several tools such as PEmer[97], Pindel[186], BreakDancer[27] and ABI SOLiD Software Tools [122].

One of the weaknesses of the standard clustering strategy is that it ignores the paired-end reads that have more than one mapping position[123], i.e., it does not allow the detection of SVs in the repetitive region including low copy regions generated by segmental duplications and transposable insertions. However, due to a strong association between segmental duplication and copy-number variation [32, 89], it is necessary to consider paired reads with multiple mapping positions in order to detect SVs within the duplicate regions. Several approaches such as variationHunter [67] and MoDIL [103] were developed to address this problem by assigning each good mapping read pair to only one cluster where it has the most support from other read pairs.

There are two parameters that define a cluster in the standard clustering strategy: one is the minimum number of paired reads required for a cluster, and the other is 
the number of standard deviations after which paired reads are considered discordant. Another weakness of the standard clustering strategy is that it uses a fixed cutoff for the number of standard deviations to determine discordant PEMs. This method can miss some clusters whose insertion size is small. For example, all paired reads at an insertion location have mapping distance of 2 standard deviations away from the mean insert size. The cluster cannot be formed if the discordance threshold is set at 3 standard deviations. Lee and colleagues [103] developed a distribution-based clustering approach, called MoDIL, to detect small insertion and deletion. This method compares the distribution of insert size in the sequenced library to the distribution of the observed mapped distances at a given location on the genome. If they match the typical insert size distribution but with a shift, then a cluster is formed. Similar approaches have been adopted in the BreakDancer [27] and ABI SOLiD Software Tools [122].

\subsubsection{Depth of coverage(DOC)-based methods}

The high coverage of NGS makes it possible to identify SVs, mainly duplications and deletions based on the depth of the coverage (DOC). SVs can be detected through the detection of DOC signatures by analyzing sequence read depths [50]. Assuming that the sequencing coverage is about the same across the entire genome, the number of reads mapped to a region is expected to be proportional to the number of times the region appears in the genome. If a region has insertion or deletion, there will be either more or less reads mapped to it. This gain/loss signature is directly related to the depth of coverage and to the size of the insertion or deletion. However, in reality, the sequencing coverage may not be uniform across the genome, and it can be over or under sampled in certain regions of the genome, which may cause the detection of false SVs.

To overcome this type of noise, current methods group DOC signatures that support the same variation into windows [123]. They first partition the reference genome into nonoverlapping windows based on the depth of coverage. Within each window, the depth of coverage is consistent, but the adjacent windows have a sudden change in the coverage of depth. Each window will correspond to a single loss, gain or no 
event. Similar to the aCGH technique, two studies $[22,28]$ used binary circular segmentation and local change point analysis techniques to find the proper breakpoint for the windows. They then used theoretical analysis and simulations to determine the minimum size of the windows that correspond to SVs. Although these methods are able to detect the insertion of duplicate sequence, they are not able to detect the insertion of novel sequence.

\subsubsection{Transposon insertion polymorphisms}

Transposon insertion polymorphism (TIP) is one type of SVs. It is defined as the presence and absence of a transposon insertion at a specific genomic location. Transposons are discrete pieces of DNA that can move within genomes [34]. They were discovered by Barbara McClintock in 1948 [121], for which she was awarded a Nobel prize in 1983. Transposons have the remarkable ability to transpose or duplicate themselves to other regions of the genome, and this process has been occurring in virtually all organisms. As a result, transposons are a major component of human and non-human primate genomes. The completion of the first human genome sequence revealed that transposons constitute nearly half of the human genome [75, 163]. Considering the protein-coding regions account for only $1.5 \%$ of the human genome, the contribution of transposons to the human genome is much more remarkable [75]. Transposons play important roles in the evolution of the genome and human genetic diversity. They can mutate the genomes by moving around to different positions in the genome, and some of these mutations have been documented to cause human diseases including cancers $[12,132]$.

Transposons are divided into two classes based on how they transpose themselves within the genome. DNA transposons, accounting for $2.8 \%$ of the human genome [34] (see Figure 3.4), can be cleaved from one site and inserted into a new site. Retrotransposons, accounting for nearly $42 \%$ of the human genome (see Figure 3.4), can copy themselves into RNA, and then reverse-transcribe into DNA and inserted at new genomic locations. Retrotransposons can be subdivided into two groups distinguished by the presence or absence of long terminal repeats (LTRs). Engoenous retrotrovirus (ERVs) are the main types of LTRs in the human genome, and they account for 
8.3\% of the genome. Most common Non-LTR retrotransposons are Alu elements and LINE-1s (L1), which account for one-third of the human genome [34] (see Figure 3.4). L1, Alu, and SVA elements (possibly HERV-K elements) are the TEs that have been shown to be currently active in humans [126]. Past and ongoing activities of these TEs generate TE insertions that differ between different populations, family lineage or even different members of the same families. So far, close to 3000 TIPs have been documented in the database of retrotranposon insertion polymorphism (dbRIP) [178]. More than 60 cases of de novo insertions were reported to be responsible for genetic disorders [34].

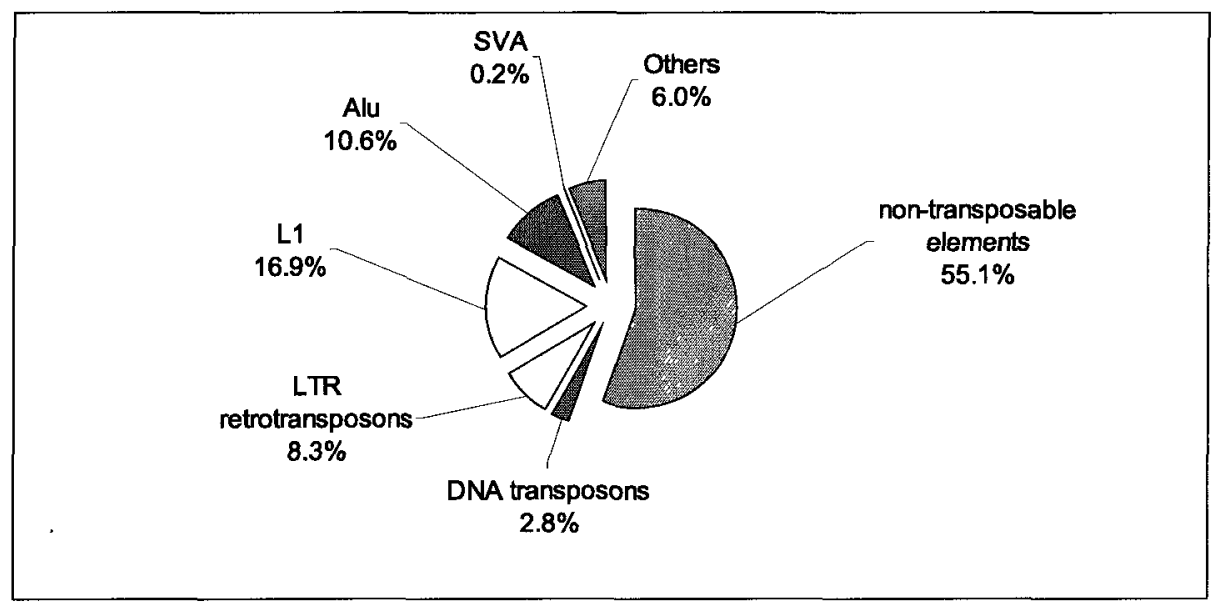

Figure 3.4: The TE content of the human genome.

Each of these major types of retrotransposons can be divided into subfamilies based on diagnostic nucleotide sequence that serve as markers to indicate a common ancestor among subfamily members. For example, more than 200 Alu subfamilies are classified and recognized $[176,138]$. The formation of the subfamilies, which often exist in a hierarchical structure, reflects the evolutionary dynamics of retrotransposon amplification in a sequential or linear accumulation fashion. For Alu Y family, new subfamilies are named using the number of nucleotide change relative to the Alu Y consensus sequence. For example, Alu Ya4 contains four nucleotide variations from the $\mathrm{Y}$ consensus. Alu Ya5 contains those same four changes plus one additional change. Letters following $\mathrm{Y}$ specify a particular cluster of additive changes that track the evolution of the active elements. Subfamily structure exists for L1, SVA and 
HERV as well[34].

\subsubsection{Computational methods for TIPs detection}

Although various computational approaches have been developed to detect SVs [123], most of them are not capable of detecting transposon insertions, which is a very important type of SVs to the study of human evolution and disease. With the completion of the human genome project, a few studies have used computational comparative genomic approaches to identify TIPs [177, 96, 179, 185], but these methods are limited to compare two assembled sequences. Very recently, Hormozdiari and colleagues [69] developed a method called variationHunter to detect transposon insertion. This is the only tool so far that identifies the TIPs by comparing the test genome and the reference genome based on NGS data.

In the variationHunter method, all discordant paired-end reads with one end mapped to the reference genome and the other end mapped to the TE consensus sequences are first collected, and then based on the mapping information of each paired-end read, the breakpoint intervals (the range of insertion locations) are computed. Note that here all reads can be mapped to multiple locations on the reference genome. After sorting all breakpoint intervals in ascending order based on their locations, a clustering algorithm is used to find the maximal valid clusters. As per [67], a valid cluster is defined as a set of alignments of discordant paired-end reads that support the same potential structural variation. A maximal valid cluster is defined as a valid cluster where no additional pair-end read alignments can be added such that the cluster remains valid. That is, a maximal valid cluster is a valid cluster for which no valid superset exists. Note that each paired-end read can be mapped to multiple locations on the reference genome. Since these locations are included in multiple maximal valid clusters, transposon insertions found from the maximal valid clusters can be incorrect. In order to reduce noise, Hormozdiari and colleagues used an approximate algorithm [67] to select the minimum number of the clusters from the maximal valid clusters such that each paired-end read has a map location in at most one selected valid cluster. They tested the variationHunter method with simulated data, by generating paired-end reads from the Venter genome assembly (HuRef) 
with the genome coverage at $10 \mathrm{X}$. After comparing with the reference genome, they discovered $>85 \%$ of transposon insertion events with a precision of $>90 \%$.

\subsection{Methods}

\subsubsection{General approach}

In this study, we focus on TIPs representing insertions that are present in the test genome but absent in the reference genome (TIPs_OUT hereafter), since they are more difficult to identify than those insertions that are present in the reference genome but absent in one or more test genomes, which we call "TIPs_IN". For paired-end reads with their fragment spanning the boundaries of the insertions, we can expect that one read-mate will map to the regions flanking the insertion with relatively good reliability (i.e. low number of hits with perfect or low number of mismatches) unless the flanking regions are repetitive in nature, while the other read-mate that falls into the transposon insertion will have a random match to a similar transposon sequence in the genome. This is because the transposon insertion is missing from the reference genome at that particular position, but an alignment may be found with other similar tranposon sequences in the genome. In this case, the alignment location of that read is a random pick among all top hits by programs such as MAQ [107] and BWA [106]. Sequence alignment data used in this study were obtained using the MAQ software . MAQ labels concordant reads with a flag of 18 (MF18 reads) and those mapped to two different chromosomes with a flag of 32 (MF32 reads). As there are 23 chromosomes in the genome, the possibility of the second read (in the transposon insertion) mapped to the same chromosome as the first read is on average at $4-5 \%$. Therefore, most of those paired-end reads would have the two reads mapped to different chromosomes (i.e. MF32 reads), and they are further characterized by having one of the reads mapped to a transposon location in the reference genome. Reads with these mapping signatures are the basis of our approach for identifying TIPs_OUT in our software, TIP-finder.

TIP-finder uses a greedy algorithm to identify the candidate TIPs_OUT loci and then predicts their insertion site, insertion size, transposon subfamily and insertion 
genotype. The TIPs_OUT candidate list is subsequently filtered by a set of parameters, which are optimized through a machine learning approach based on a simulated data containing known TIPs_OUT documented in dbRIP database [178].

\subsubsection{Identification of TIPs_OUT candidate loci}

We developed a greedy algorithm called TIPs_OUTclusterFinding to identify the TIPs_OUT candidate loci. TIPs_OUT candidate reads are defined as paired reads with one read in the flank region and another in newly inserted transposon loci. Here, we denote the sets of TIPs_OUT candidate reads as $R=\left(A_{1}, B_{1}\right),\left(A_{2}, B_{2}\right), \cdots,\left(A_{n}, B_{n}\right)$, where $A_{\imath}$ represents the reads in the flank region and $B_{\imath}$ represents the reads in inserted transposon loci as shown in Figure 3.5. The sets of all alignments of $\left(A_{\imath}, B_{\imath}\right)$ mapped to the reference genome are defined as Align $=\operatorname{align}_{1}$, align $_{2}, \cdots$, align $n$. Note that each alignment contains the mapped locations to the reference and the alignment orientations. That is, $\operatorname{allgn}_{\imath}=\left(\operatorname{pos} A_{\imath}, \operatorname{pos} B_{\imath}, \operatorname{or} A_{\imath}, \operatorname{or} B_{\imath}\right)$ where $\operatorname{pos} A_{\imath}$ and $\operatorname{pos} B_{\imath}$ represent the mapping locations of $A_{\imath}$ and $B_{\imath}$ on the reference genome and or $A_{\imath}$ and $\operatorname{or} B_{\imath}$ represent the mapping orientation of both end reads. The mapping orientation of the reads can be either "+" (forward strand) or "_" (reverse strand).

Based on alignments of paired-end reads to the reference genome using MAQ, TIPs_OUT candidate reads are characterized by having the two reads mapped to different chromosome, i.e. MF32 reads. Further, we require that one of the reads mapped to a non-transposon position and the other mapped to a transposon position. The location of transposons in the reference genome is based on the RepeatMasker annotation obtained from the UCSC Genome Browser (http://genome.ucsc.edu). Figure 3.5 shows an example of a TIPS_OUT candidate locus, while the algorithms and pipelines included in our TIP-finder tool are described below.

\section{Algorithm 7 TIPs_OUTclusterFinding}

(1) identify and collect all paired reads with mapping flag 32 (readMF32).

(2) identify TIPs_OUT candidate reads $\left(A_{\imath}, B_{\imath}\right)$

(a) map all readMF32 to a sorted transposon position list on the reference genome. 


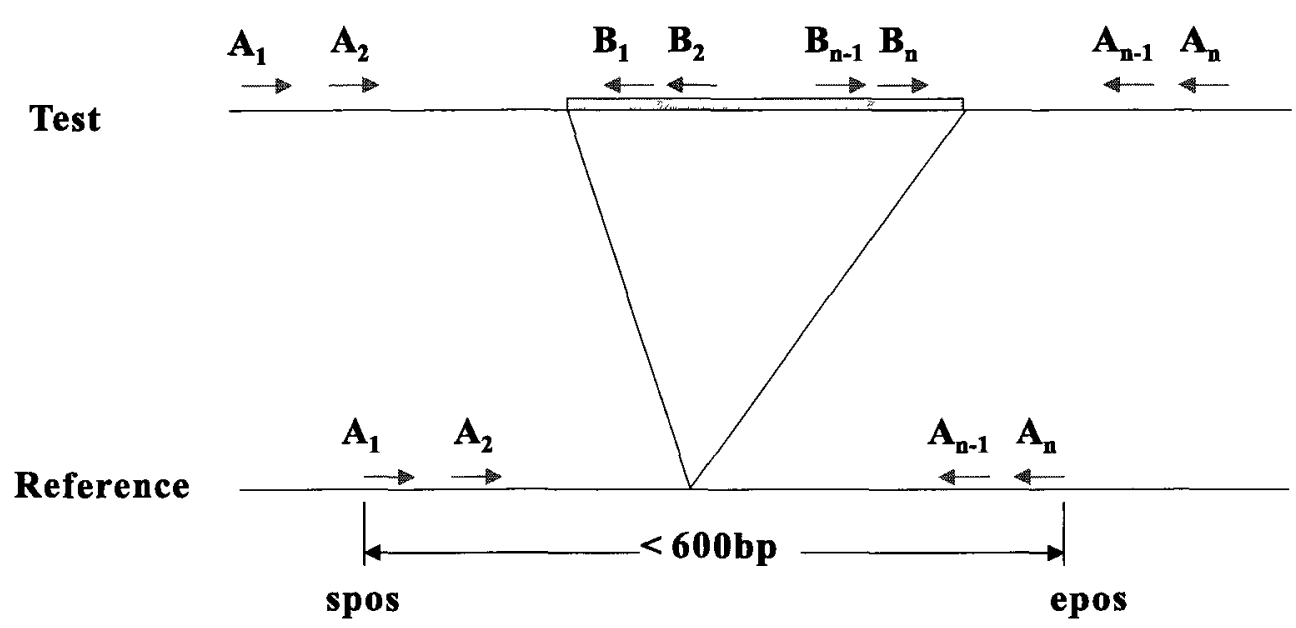

Figure 3.5: An example of a TIPs_OUTcandidate locus. $A_{i}$ represents the read mapped to the reference genome in a non-transposon position. $B_{i}$ represents the read mapped to a transposon position. Library size for our data set is around $250 \mathrm{bp}$ with a standard deviation of $100 \mathrm{bp}$. The minimum and maximum positions of all $A_{i}$ are set as the minimum (spos) and maximum (epos) positions for the locus. Note that $B_{n-1}$ and $B_{n}$ can also fall before $B_{1}$ and $B_{2}$ when the insertion is relatively short.

(b) for $A_{\imath}$, if it is not mapped to the transposon positions but its pair-mate $B_{i}$ is, add $\left(A_{\imath}, B_{i}\right)$ into the TIPs_OUT candidate reads list $L$.

(3) sort the list $L=\left[\left(A_{1}, B_{1}\right),\left(A_{2}, B_{2}\right), \cdots,\left(A_{n-1}, B_{n-1}\right),\left(A_{n}, B_{n}\right)\right]$ according to the locations of $A_{i}$ on the reference genome (pos $A_{i}$ )

(4) cluster $\left(A_{i}, B_{i}\right)$ based on $\operatorname{pos} A_{i}$

(a) If $\left|p o s A_{1}-p o s A_{2}\right|<2 \times$ LibrarySize and $B_{1}$ and $B_{2}$ are mapped to the same transposon family, $\left(A_{1}, B_{1}\right)$ and $\left(A_{2}, B_{2}\right)$ are grouped into one cluster.

(b) if the number of paired reads in a cluster is less than 2, discard the cluster. (4) find the minimum (spos) and maximum (epos) positions of $A_{2}$ for each cluster. - End of Algorithm -

Tables 3.2 lists the runtime cost of each step of the TIPs_OUTclusterFinding algorithm. In this table, $N$ is the number of readMF32, and $T$ is the number of annotated transposons in the reference genome. For simplicity, we will ignore step 1 and assume all readMF32 have already been loaded in a sorted list (alignments of paired-end reads are sorted based on their mapping positions by MAQ). In step 
$2 \mathrm{~b}$, to identify TIPs_OUT candidate reads, each readMF32 needs to find whether its pair-mate is mapped to a transposon position or not. This is processed by a binary search and the time complexity for it is $O(N \log N)$. In step 3, the worst case for the TIPs_OUTclusterFinding algorithm is that all readMF32 reads are candidate reads, so the time complexity for this step is $O(N \log N)$. The total time complexity of the TIPs_OUTclusterFinding algorithm is given by:

$$
\begin{aligned}
& =O(N+T+N \log N)+O(N \log N)+O\left(N^{2}\right)+O(N)+O(N) \\
& =O\left(N+T+N \log N+N^{2}\right)
\end{aligned}
$$

\begin{tabular}{lr}
\hline Steps & Costs \\
\hline step 2a & $O(N+T)$ \\
step 2b & $O(N \log N+N)$ \\
step 3 & $O(N \log N)$ \\
step 4a & $O\left(N^{2}\right)$ \\
step 4b & $O(N)$ \\
step 5 & $O(N)$ \\
\hline
\end{tabular}

Table 3.2: List of the time complexity of the TIPs_OUTclusterFinding algorithm.

\subsubsection{Prediction of the insertion location}

The cluster found by Algorithm 7 is a cluster of discordant paired-end reads which support the same potential transposon insertion. Clustering not only helps to improve confidence in the predictions of TIPs, but also to increase the precision of the predicted insertion breakpoints and insertion size. As shown in Figure 3.5, the insertion breakpoint is in the range of minimum(spos) and maximum(epos) positions of the cluster. Ideally, the orientation of $A_{\imath}$ in the cluster should be in an order as " $\rightarrow \rightarrow \rightarrow \leftarrow \leftarrow \leftarrow$ ", and the insertion location is the position where $A_{\imath}$ changes its orientation from forward to reverse. However, due to the problems of read sampling and mapping, some paired-end reads do not show correct orientation patterns. Therefore, it is hard to predict an exact insertion position. In this study, we use the algorithm described below to predict the minimum range of the insertion location ([isite_spos, isite_epos]) which is based on the mapping position $\left(p o s A_{\imath}\right)$ and orientation $\left(\right.$ or $A_{2}$ ) of $A_{2}$. [isite_spos, isite_epos] computed in Algorithm 8 is the minimal 
region of $A_{\imath}$ which does not necessarily follow a correct orientation pattern.

Algorithm 8 TIPs_OUTinsertionSite

$$
\begin{aligned}
& \text { left } \leftarrow 0 \\
& \text { right } \leftarrow 0 \\
& \text { isite_spos } \leftarrow \text { spos } \\
& \text { isite_epos } \leftarrow \text { epos } \\
& \text { for } \mathrm{i} \text { from } 0 \text { to } \mathrm{n}
\end{aligned}
$$

$$
\begin{aligned}
& \text { if } \text { or } A_{\imath}="+" \\
& \quad \text { if right }=1 \text { right } \leftarrow 0 \\
& \quad \text { if left }=0 \text { isite_spos } \leftarrow \text { pos } A_{\imath}
\end{aligned}
$$

else

$$
\begin{aligned}
& \text { if left }=0 \text { left } \leftarrow 1 \\
& \text { if right }=0 \\
& \quad \text { isite_epos } \leftarrow \text { pos } A_{\imath} \\
& \text { right } \leftarrow 1
\end{aligned}
$$

- End of Algorithm -

\subsubsection{Prediction of the insertion size and orientation}

A transposon insertion can be classified as insertion split or insertion overlap for predicting the size of the insertion as described below. Here, we only consider the first and last paired reads for each insertion locus. When the paired reads have an orientation pattern as $A_{1}(\rightarrow), \cdots, B_{1}(\leftarrow), \cdots, B_{n}(\rightarrow), \cdots, A_{n}(\leftarrow)$, we define this insertion as insertion split (see Figure 3.6). It indicates that the $B$ reads of the first and the last PEM pairs locate at the left and right end of the insertion, respectively, leaving a distance between the two $\mathrm{B}$ reads in the insertion. This happens when the insertion size is much larger than the sequence library insert size. When the paired reads have an orientation as $A_{1}(\rightarrow), \cdots, B_{n}(\rightarrow), \cdots, B_{1}(\leftarrow), \cdots, A_{n}(\leftarrow)$ (see Figure 3.7), we define this type of insertions as insertion overlap. It indicates that two DNA fragments represented by the first and last paired reads overlap in the insertion 
region, and it happens when the insertion size is close to or smaller than the size of library fragments.

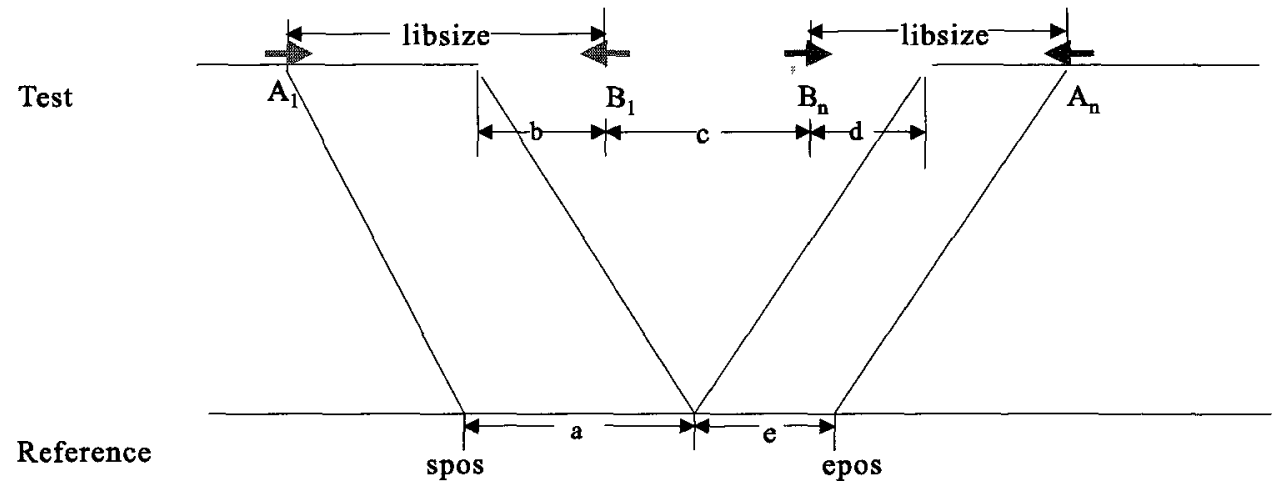

Figure 3.6: Schematic representation of an insertion split. In the case of an insertion split, the B reads of the first and the last PEM pairs locate at the left and right end of the insertion, respectively, leaving a distance between the two B reads in the insertion. This happens when the insertion size is much larger than the sequence library insert size.

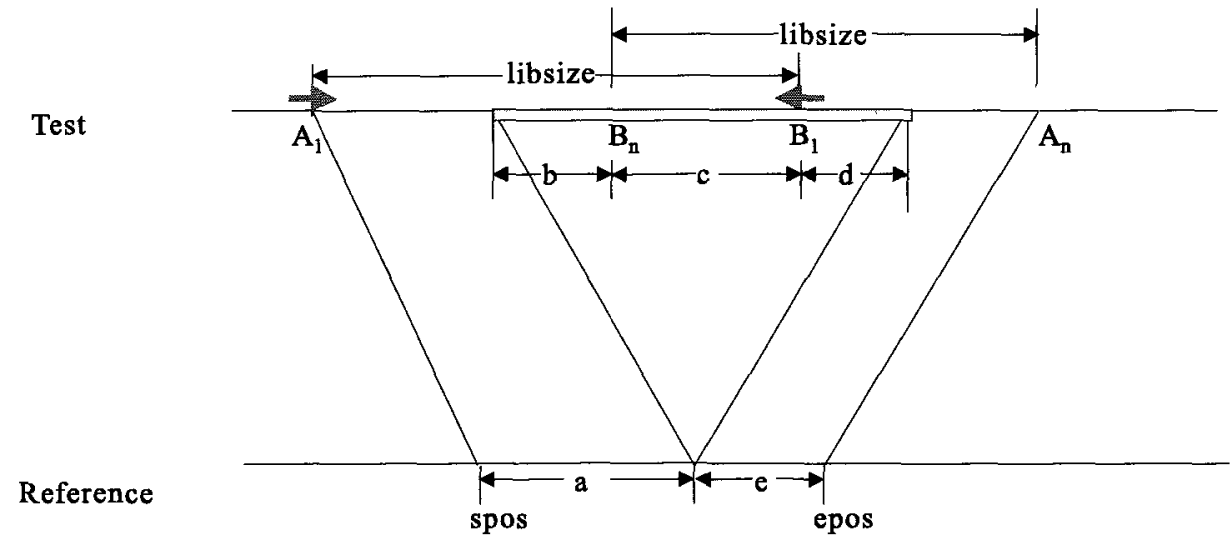

Figure 3.7: Schematic representation of an insertion overlap. In the case of an insertion overlap, two DNA fragments overlap in the insertion region, and it happens when the insertion size is close to or smaller than the size of library fragments.

The paired-end reads are sequenced from two ends with head to head $(\rightarrow \leftarrow)$ 
orientations in the case of Illumina PEM. When they are mapped to the reference genome, reads in the left flank region (e.g. $A_{1}$ in Figure 3.5) should be mapped to the forward orientation ("+") and reads in the right flank region (e.g. $A_{n}$ in Figure 3.5) should be mapped to the reverse orientation ("-"). Since the transposon insertion is missing from the reference genome at the insertion position, the reads representing the inserted transposon element can be mapped to other locations on the reference genome either in a forward orientation or a reverse orientation. Note that the alignment data we used in this study are in a BAM format, which reports all mapped reads based on the forward genomic strand. To change the reads to their original orientation in the test genome, we perform the following based on the strand information provided in BAM: if a read is in a reverse strand ("_), then it is reverse complemented; if a read is a forward strand, then it is kept unchanged.

Although TE reads in the same transposon insertion can be mapped to different locations on the reference genome, they should be mapped to the same transposon consensus sequence with the highest score. Thus, the mapping positions and orientations of $\mathrm{TE}$ reads on the transposon consensus sequence can be used to determine the mapping orientation of the inserted transposon in reference to the reference genome. For example, $B_{1}$ and $B_{n}$ in Figure 3.6 have different orientations in the test genome, so when both of them are mapped to the corresponding transposon consensus sequence, the mapping orientations of $B_{1}$ and $B_{n}$ should be different as well. Since $B_{n}$ has a forward orientation to the reference genome, if it is mapped to the transposon consensus sequence in a forward orientation, then the inserted transposon should have a forward orientation to the reference. Otherwise, it should have a reverse orientation. Based on the mapping positions of read $B_{1}\left(p o s T E \_B_{1}\right)$ and $\operatorname{read} B_{n}\left(p o s T E_{-} B_{n}\right)$ in the transposon consensus sequence and the mapping orientation of inserted transposon (orTE), insertion type can be identified as follows:

(1) if orTE $=$ "+" and $\operatorname{pos} T E_{-} B_{1}<\operatorname{pos} T E \_B_{n}$, it is insertion split.

(2) if orTE $=$ "+" and $\operatorname{pos} T E_{-} B_{1}>\operatorname{pos} T E_{\_} B_{n}$, it is insertion overlap.

(3) if orTE = "-" and posTE_B $B_{1}<\operatorname{pos} T E_{-} B_{n}$, it is insertion overlap.

(4) if orTE $=$ "-" and posTE_B $B_{1}>$ posTE_ $B_{n}$, it is insertion split.

In the case of insertion split (see Figure 3.6), the insertion size is calculated as follows: 


$$
\begin{aligned}
\text { insertion_size } & =b+c+d \\
& =(\text { libsize }-a)+\operatorname{distanceTest}\left(B_{1}, B_{n}\right)+(\text { libsize }-e) \\
& =2 \times \text { libsize }-(a+e)+\operatorname{distanceTest}\left(B_{1}, B_{n}\right) \\
& =2 \times \text { libsize }-\operatorname{distanceRef}\left(A_{1}, A_{n}\right)+\operatorname{distanceTest}\left(B_{1}, B_{n}\right)
\end{aligned}
$$

In the case of insertion overlap (see Figure 3.7), the insertion size is calculated as:

$$
\begin{aligned}
\text { insertion_size } & =(b+c)+d \\
& =(\text { libsize }-a)+(\text { libsize }-c-e) \\
& =2 \times \text { libsize }-(a+e)-\operatorname{distanceTest}\left(B_{1}, B_{n}\right) \\
& =2 \times \text { libsize }- \text { distanceRef }\left(A_{1}, A_{n}\right)-\operatorname{distanceTest}\left(B_{1}, B_{n}\right)
\end{aligned}
$$

where libsize is the library size, distance Re $f\left(A_{1}, A_{n}\right)$ is the distance between reads $A_{1}$ and $A_{n}$ on the reference genome, which is also the size of the locus (see Figure 3.6). distanceTest $\left(B_{1}, B_{n}\right)$ is the distance of $B_{1}$ and $B_{n}$ on the test genome, which is also the distance of $B_{1}$ and $B_{n}$ on the inserted transposon sequence. To compute this distance, we first map reads $B_{1}$ and $B_{n}$ to all transposon consensus sequences using BLAST, and then choose the best matched common consensus sequence as a reference sequence to determine the distance between $B_{1}$ and $B_{n}$. However, this method can only be used to predict the insertion size of the TIPs_OUT loci whose paired reads have ideal mapping orientations, i.e. the paired reads that meet the following conditions: 1) $A_{1}$ and $A_{n}$ are mapped to the reference genome in forward and reverse orientations, respectively; 2) $B_{1}$ and $B_{n}$ are mapped to the same TE consensus sequence; 3) $B_{1}$ and $B_{n}$ have different mapping orientations to the TE consensus sequence. We label these three conditions as flags: PF, TF and SF. If a locus meets a condition, the value of the corresponding flag will be set as 0 , otherwise, it will be set as 100 .

\subsubsection{Prediction of the insertion transposon subfamily}

The transposon subfamily of the insertion is predicted based on the most dominant subfamily among all MF32 reads $\left(B_{i}\right.$ in Figure 3.5$)$ for the predicted transposon insertion. The prediction method is shown in Algorithm 9. As discussed in Section 3.3.1, since the transposon insertion is absent in the reference genome, the reads in the transposon insertion $\left(B_{i}\right.$ in Figure 3.5) will have a random match to a similar transposon 
sequence in the reference genome and their alignment locations will be a random pick among all top hits by the MAQ software. So the mapping locations of these reads are unreliable, and thus they cannot be used to determine the insertion transposon subfamily by comparing the transposon locations in the reference genome and their corresponding transposon subfamilies documented in the RepeatMasker database.

In this study, in order to predict the insertion transposon subfamily, the B reads in the insertion are mapped to the consensus sequences of all transposon subfamilies by the BLAST software [4], a widely used bioinformatics tool. Note that a read can be mapped to multiple subfamilies with the same highest score if the B reads fall into a region in the consensus sequences where there is no difference among subfamilies. In this case, all the best matched subfamilies for each B read are recorded and the most dominant subfamily among all B reads is chosen as the transposon subfamily of the insertion. The consensus sequences of all transposon subfamilies were obtained from Repbase [80] (http://www.girinst.org).

Algorithm 9 Identification of subfamily for a TIPs_OUT candidate locus

(1) Set the initial value for all transposon subfamilies $\left(T E_{\jmath}\right)$ as 0.

(2) Map all readMF32 $B_{\imath}$ of the insertion to the consensus sequences of $T E_{\jmath}$ by BLAST.

(3) If $B_{\imath}$ is mapped to a subfamily $T E_{\jmath}$ with the best score, increase value $T E_{\jmath}$ by 1 .

(4) Find the maximum number of valueTE$E_{\jmath}$.

(5) The TE subfamily with the maximum value is chosen as the subfamily assignment of this insertion.

- End of Algorithm -

\subsubsection{Prediction of the insertion genotype}

The genotype of the insertion in the test genome can also be predicted. Human somatic cells have a diploid genome containing two copies of each chromosome. Therefore, a TE insertion in a genome can exist in one of the three forms or genotypes, i.e. present in two copies, present in one copy or absent from both copies, and they are denoted as "+/+", "+/-" and "-/-", respectively. For the TIPs_OUT predicted in a 
given genome, it can be either " $+/+$ " or " $+/-$ ". For genotype " $+/+$ ", most pairedend reads in the region flanking the insertion display a flag MF32 and very few or no reads are concordant pairs (a flag of MF18), while for genotype "+/-", about half of the pair reads display a flag of MF32 and the other half show a flag of MF18.

The theoretical ratio of numMF18/numMF32 should be 0 and 1 for the " $+/+$ " and " $+/-$ " genotype, respectively. In this study, a simulation is used to determine the optimal cut off value for defining each genotype as shown in Table 3.3(see details in Section 3.3.7). It can be seen that when the ratio of numMF18/numMF32 is less than 0.8 , the genotype of insertion is defined as " $+/+$ "; otherwise, when the ratio of is equal to or greater than 0.8 , the genotype is defined as "+/.". We also defined an upper boundary cut off for the genotype of "+/-". As shown in Table 3.3, when the ratio of numMF18/numMF32 is greater than 3, the genotype will change from " + /-" to "-/-". The upper boundary cut off value for the genotype of " $+/-$ " was determined by the method describe below.

\subsubsection{TIPs_OUT loci filtering}

In order to reduce false positives (FP) in predicting TIPs_OUT, the predicted TIPs_OUT candidate loci are filtered by imposing restrictions on numMF32, the ratio between numMF32 and numMF18, and the percentage of MF32 reads ( $A_{i}$ in Figure 3.5) showing reliable mapping in the flank region of TIPs_OUT (percentReliableMF32). To optimize these filtering parameters, a machine learning approach was used based on a simulated data containing known TIPs_OUT documented in dbRIP database [178]. dbRIP is a database of human Retrotransposon Insertion Polymorphisms (RIPs), and it contains all currently known Alu, L1, HERV and SVA polymorphic insertion loci in the human genome. In simulating the known TIPs_OUT based on the dbRIP database, we inserted the 781 TIPs_OUT (695 Alus, 1 HERV, 13 SVAs and 72 L1s) from dbRIP into the reference genome at their corresponding locations to generate a simulated diploid genome sequence with one copy containing all of these TIPs_OUT and the other copy containing randomly selected $50 \%$ of the TIPs_OUT. Therefore, $50 \%$ of these TIPs_OUT are in the " $+/+$ " genotype, while the remaining $50 \%$ are in the "+/-" genotype. 
Based on the sequences of these two copies of the simulated genomes, we generated paired-end reads with a read length of $35 \mathrm{bp}$ (similar to the Illumina Solexa platform) at a size of $260 \mathrm{bp}$ with $60 \mathrm{bp}$ as the deviation using the sequence simulation utility included in the MAQ package [107]. These reads were then aligned to the reference genome (NCBI Buid 36/UCSC hg18) using MAQ and the output was converted to BAM/SAM format. Different amounts of sequence data were used to simulate genome coverage at $5 \mathrm{X}, 11 \mathrm{X}, 22 \mathrm{X}, 26 \mathrm{X}, 32 \mathrm{X}$ and $46 \mathrm{X}$. Note that for a simulated genome whose length, total number of reads and read length are all known, genome coverage can easily be computed using the formula presented in Section 3.2.3. For the 781 TIPs_OUT inserted in the simulated genome, we divided them into two disjoint sets: the first set (dbRIP_setA) contains 391 TIPs_OUT and the second set (dbRIP_setB) contains 390 TIPs_OUT. dbRIP_setA is used to train our algorithms by identifying the data patterns surround the insertion sites, and it is also used to determine the optimal combinatorial parameters in filtering the noise. dbRIP_setB is used to assess the performance of our algorithms by using the filtering parameters generated from dbRIP_setA. Since large-scale data are involved in the simulation, we used computer clusters for all computation and data analysis.

If a read is mapped to many positions in the genome, it is very likely that this read is in a repetitive region in the genome, and its mapping position is likely a random pick among all possible matches, thus the prediction is unreliable and may lead to false positive. To filter out these false positives, we added percentReliableMF32 as a filtering parameter. In the MAQ alignment data, flag $\mathrm{H} 0$ and $\mathrm{H} 1$ are the number of perfect hits and hits with one difference, respectively. In this study, the mapping of a read is considered as unreliable under any one of the following conditions: 1) $H 0>$ 4; 2) $H 0=0$ and $H 1 \geq 10 ; 3) H 0=0$ and $H 1=0$. percentReliableMF32 for each insertion is computed using Algorithm 10 and TIPs_OUT are filtered out when percentReliableMF32 is less than $80 \%$. This excludes TIPs_OUT in the repetitive sequence regions which tend to produce high level of false positive. We choose $80 \%$ as the cutoff for percentReliableMF32, which is the average of mean $-3 \times S D$ for the training data set at different sequencing coverage (data not shown). 
Algorithm 10 Computing percentReliableMF32

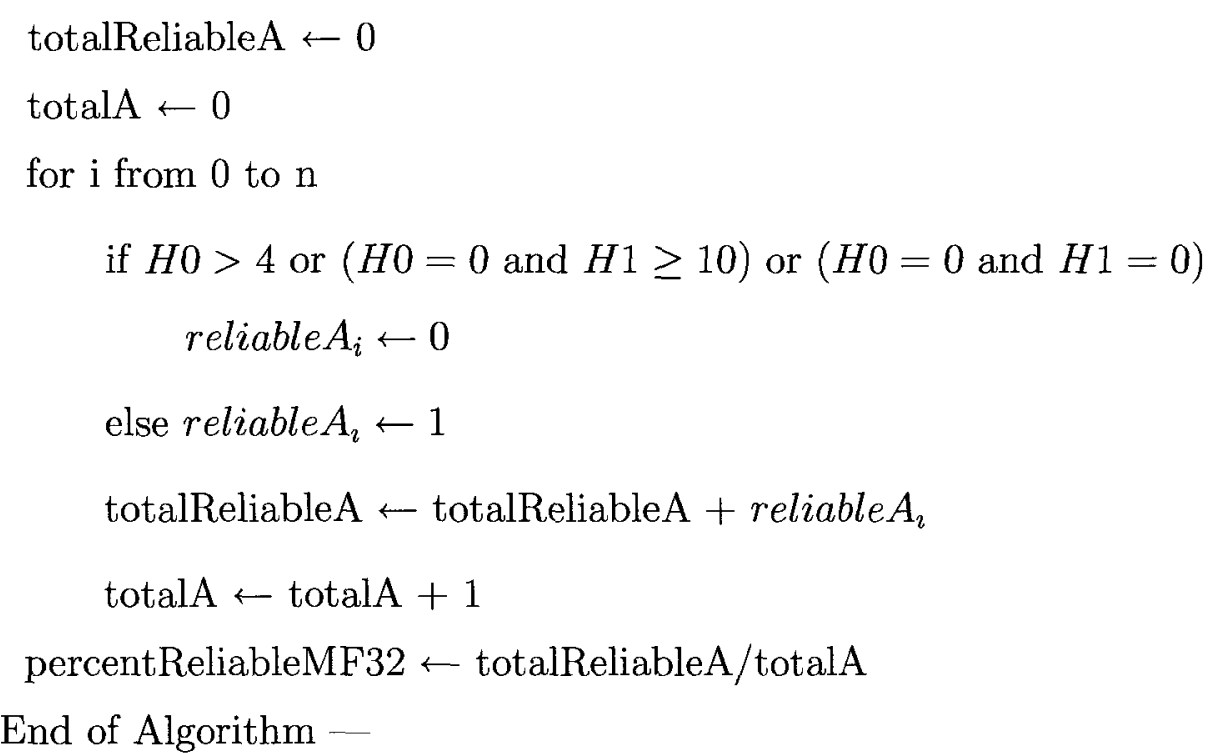

To optimize other filtering parameters, we first used steps 1 and 2 presented in Algorithm 7 to identify all MF32 candidate reads. At each known transposon insertion site of dbRIP_setA, we collected all MF32 candidate reads and all MF18 reads within a distance of the library size to the insertion site. We then calculated their numMF32 and numMF18. As discussed before, the genotype is mainly determined by the ratio of numMF18/numMF32. To define a genotype, we need to determine two cut off values for numMF18/numMF32: cutoffA and cutoffB. cutoffA is the upper boundary cutoff value for genotype " $+/+$ " as well as the lower boundary cutoff value for genotype "+/-", while cutoffB is the upper boundary cutoff for genotype "+/-" and lower boundary cutoff for genotype "-/.". Since the inserted locations of the dbRIP_setA and their genotypes are known, cutoffA was searched iteratively so that the best prediction of the genotypes at all inserted locations can be achieved. The simulated optimal values of cutoffA for different genome coverage are shown in Figure 3.8. It is noted that these values vary for different genome coverage, however, in theory they should be independent of the latter. Therefore, a mean value of 0.8 was chosen as cutoffA for this study. This approach cannot be used to determine cutoffB due to the lack of genotype "-/-" at these locations. In order to identify cutoffB, the ratio of numMF18/numMF32 at each inserted location was first calculated for entries of "+/-". The cutoffB for individual genome coverage was then determined as mean + 
$2.5 \times S D$, where "mean" and SD refer to the average ratio of numMF18/numMF32 and standard deviation, both excluding the top and bottom 5\% data points as the outliers. The simulated values of cutoffB for different genome coverage are shown in Figure 3.9. The average value of 3 was then used as cutoffB for this study.

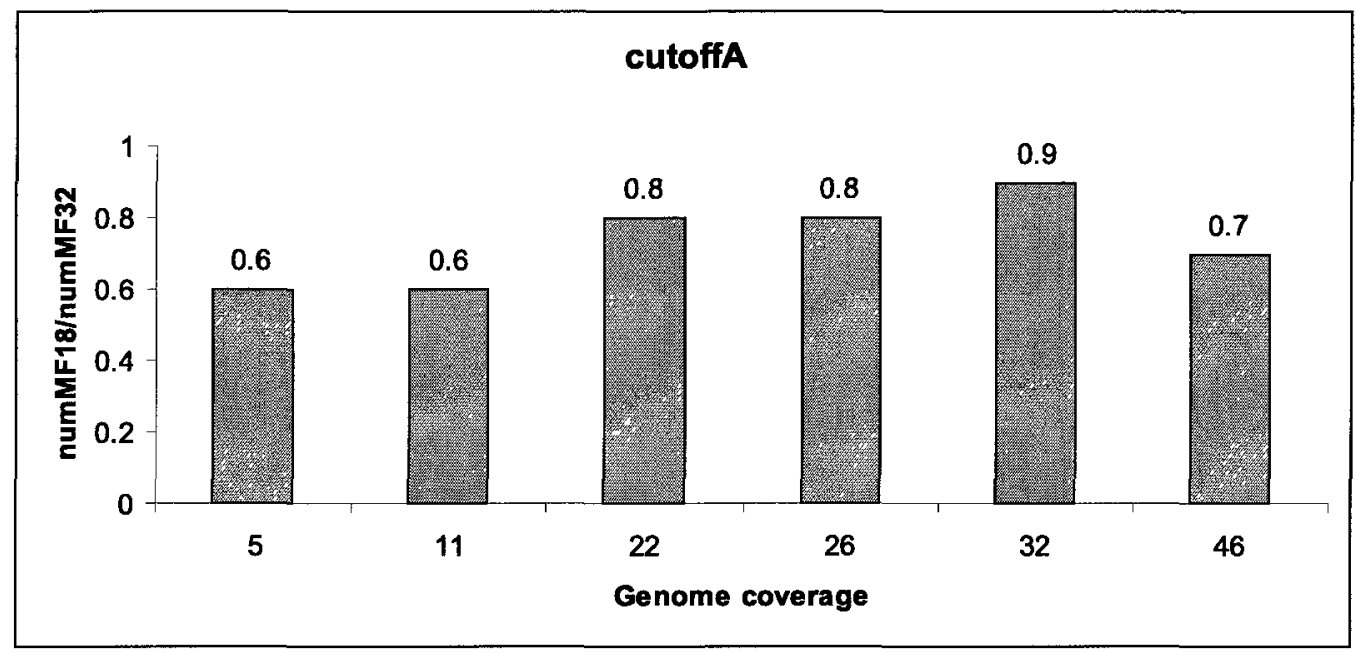

Figure 3.8: The simulated values of cutoffA for different genome coverage. cutoffA is the upper boundary cutoff value for genotype " $+/+$ " as well as the lower boundary cutoff value for genotype "+/-".

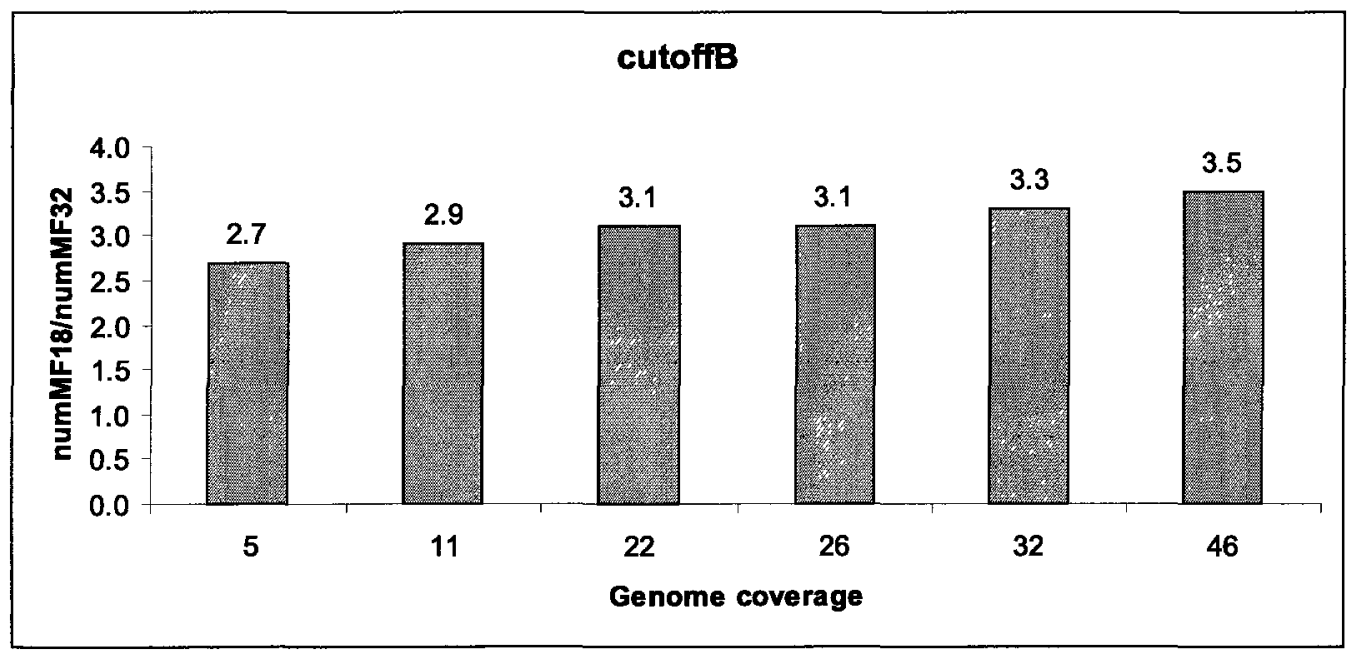

Figure 3.9: The simulated values of cutoffB for different genome coverage. cutoffB is the upper boundary cutoff value for genotype "+/-" as well as the lower boundary cutoff value for genotype "-/-". 
Minimum numMF32 is another filtering parameter. As discussed before, numMF32 in the insertion with genotype " $+/+$ " should be greater than that of genotype "+/ $"$ " Thus, different cut-off values for numMF32 should be used for different genotypes. In this study, for genotypes " + /+" and "+/-", the cut-off values for minimum numMF32 were searched iteratively so that about $95 \%$ of the known insertion sites can be correctly identified. Note that numMF32 is directly related to genome coverage. Therefore, after the cutoff values for minimum numMF32 were obtained for different genome coverage, the regression functions were further generated to show the relationship between the minimal numMF32 and genome coverage (see Figure 3.10(a) and Figure 3.10(b)). The final filtering parameters used in TIP-finder are summarized in Table 3.3.

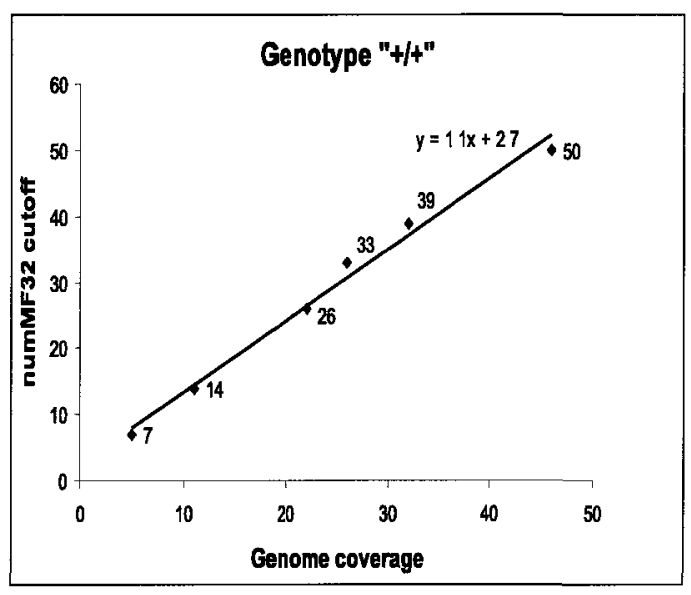

(a) Genotype "+1+"

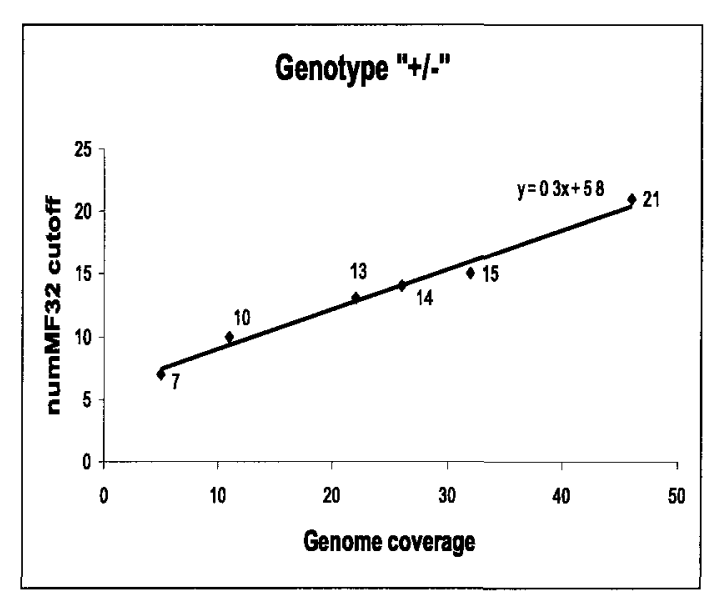

(b) Genotype "+/-"

Figure 3.10: Relationship between genome coverage and numMF32 cutoff for different genotype.

\begin{tabular}{|l|c|}
\hline & Filtering criteria \\
\hline percentReliableMF32 & percentReliableMF32 $\geq 80 \%$ \\
Genotype "+/+" & numMF $18 /$ num $M F 32<0.8$ \\
Genotype "+/-" & $0.8 \leq$ num $M F 18 /$ num $M F 32 \leq 3$ \\
numMF32 for "+/+" & numM $F 32 \geq 1.1 \times$ coverage +2.7 \\
numMF32 for "+/-" & numM $M 32 \geq 0.3 \times$ coverage +5.8 \\
\hline
\end{tabular}

Table 3.3: Summary of filtering parameters used in TIP-finder. 


\subsection{Results}

\subsubsection{TIPs_OUT identification in the simulated genome}

The simulated genome sequences contain two sets of known TIPs_OUT: dbRIP_setA and dbRIP_setB. dbRIP_setA was used to determine the optimal combinatorial filtering parameters and dbRIP_setB was used to test our algorithms. With the known insertion locations, the defined genotype and subfamily assignment, dbRIP_setB allowed us to provide an accurate assessment of the accuracy of TIP-finder in identifying TIPs_OUT in terms of both false positive and false negative rates, as well as the accuracy in predicting the TE subfamily and insertion genotype. Here, precision is calculated as percentage of true positive among all prediction, i.e. $(T P /(T P+F P))[\%]$, and sensitivity is calculated as percentage of detected true positive among all true TIPs_OUT, i.e. $(T P /(T P+F N))[\%]$, where TP, FN, and FP are the nurnber of true positive, false negative, and false positive, respectively. Since there are 390 TIPS $_{-}$OUT simulated in the genome, $(\mathrm{TP}+\mathrm{FN})$ is 390 .

As shown in Table 3.4, for genome coverage over 22X, our program is able to detect the known TIPs_OUT with sensitivity $\geq 88 \%$ and precision $\geq 91 \%$. It can be seen that the precision decreases when genome coverage increases. In fact, it is likely that the error rate associated with the alignment data increases when genome coverage grows, which could result in more false positives. The precision of TIPs_OUT prediction does not seem to be affected by the low genome coverage, while the sensitivity tends to drop slightly along with the decreasing genome coverage. The fact that a genome sequence data at a coverage as low as $5 \mathrm{X}$ still allows detection of more than $84 \%$ of true TIPs_OUT suggests that low coverage personal genome data that are currently available at a much larger number than those at deep coverage can also be valuable for the prediction of structural variations.

The accuracy of genotype prediction is above $80 \%$ for all examined levels of genome coverage as shown in Table 3.4. For subfamily prediction, the accuracy of our program is above $75 \%$ for genome at coverage over $22 \mathrm{X}$, but it decreases as the coverage drops below 22X. For insertion size prediction, we allow $20 \%$ error rate between the predicted size and the actual size. Among all TIPs_OUT with flags PF:0, TF:0 and 
$\mathrm{SF}: 0$, over $74 \%$ of the predicted insertion sizes match their actual sizes for all checked genome at coverage over $20 \mathrm{X}$. As the coverage decreases, the accuracy of insertion size predicted decreases as well.

\begin{tabular}{|l|r|r|r|r|r|r|}
\hline Genome coverage & $5 \mathrm{X}$ & $11 \mathrm{X}$ & $22 \mathrm{X}$ & $26 \mathrm{X}$ & $32 \mathrm{X}$ & $46 \mathrm{X}$ \\
\hline TP & 328 & 340 & 343 & 345 & 346 & 348 \\
TP+FP & 355 & 367 & 375 & 378 & 380 & 392 \\
TP+FN & 390 & 390 & 390 & 390 & 390 & 390 \\
Precision & $92 \%$ & $93 \%$ & $91 \%$ & $91 \%$ & $91 \%$ & $89 \%$ \\
Sensitivity & $84 \%$ & $87 \%$ & $88 \%$ & $88 \%$ & $89 \%$ & $89 \%$ \\
Genotype match & $80 \%$ & $88 \%$ & $88 \%$ & $89 \%$ & $90 \%$ & $91 \%$ \\
Subfamily match & $68 \%$ & $74 \%$ & $76 \%$ & $76 \%$ & $76 \%$ & $76 \%$ \\
Insertion size match & $50 \%$ & $63 \%$ & $74 \%$ & $74 \%$ & $74 \%$ & $76 \%$ \\
\hline
\end{tabular}

Table 3.4: Summary of TIPs_OUT predictions based on simulation.

\subsubsection{TIPs_OUT prediction based on six genomes}

To identify TIPs_OUT using TIP-finder, we downloaded genome data for six individuals representing two trio families (each consists of the two parents and a child) that were subjected to deep sequencing by the 1000 Genome Project at a coverage from $20 \mathrm{X}$ to $40 \mathrm{X}$ (http://1000genomes.org). The specific samples are NA12878 (daughter), NA12891 (mother) and NA12892 (father) for the Utah Caucasian family and NA19238 (mother), NA19239 (father) and NA19240 (daughter) for the Nigerian family. We used the alignment data of sequences generated using the Illumina Solexa platform provided in BAM format by the 1000 genome project using MAQ (http://1000genomes.org). The TIPs_OUT prediction was first performed for each genome individually and then the 6 lists of TIPs_OUT were combined to generate a non-redundant list of final TIPs_OUT candidate list (see Table 3.6). For each TIP_OUT, the location, the number of MF32 and MF18 reads, the insertion size, the insertion site, the subfamily assignment, and the genotype are provided in a table format (see Table 3.5). Note that the genome coverage is computed as the ratio between the total length of the reads and the genome length. Since the lengths of the above six genomes are unknown, in this study we used the length of the reference genome to compute the genome coverage for these six genomes. 


\begin{tabular}{|c|c|c|}
\hline Locus ID & Locus position & Prediction $^{a}$ \\
\hline 1 & chr18 $55368783-55369297$ & NA19238 $\mid$ AluSp $|55368861-55369249| 170 \mid+(-|41| 50|\mathrm{R} 0| \mathrm{P} 0|\mathrm{~S} 0|$ \\
\hline 2 & chr13 30789846-30790187 & NA12878|AluYal $|30790010-30790054| 209|+/-| 18|29| \mathrm{R}$ o|P $0 \mid \mathrm{S}$ ol \\
\hline 3 & chr10 98408418-98408699 & NA12892|AluYa5 $|98408536-98408573| 369|+/-| 15|26| \mathrm{R}$ 0|P $0|\mathrm{~S} 0|$ \\
\hline 4 & $\operatorname{chr} 738444585-38444855$ & NA12878|AluYa4|38444621-38444712|377|+/-|18|45|R 0|P 0|S 0| \\
\hline 5 & chr20 $37643996-37644270$ & NA12892|AluYb8|37644046-37644080|257|+/-|20|23|R 0|P 0|S 0| \\
\hline 6 & chr6 32697653-32697909 & NA12878|AluYa5 $|32697767-32697807| 414|+/-| 19|27| \mathrm{R} 0 \mid \mathrm{P}$ 0|S $0 \mid$ \\
\hline 7 & chr4 64409354-64409661 & NA12878|AluYc1 $|64409458-64409535| 371|+/-| 18|33| \mathrm{R}$ 0|P o|S 0| \\
\hline 8 & chr1 58977748-58978054 & NA12878|AluYa4 $|58977863-58977898| 380|+/-| 18|32| \mathrm{R}$ 0|P 0|S $0 \mid$ \\
\hline 9 & chr8 110170603-110170917 & NA12878 $\mid$ AluYe5 $|110170744-110170808| 380|+/-| 25|44| \mathrm{R} 0|\mathrm{P} 0| \mathrm{S} 0 \mid$ \\
\hline 10 & chr1 176761629-176762154 & NA19240|AluYg6 $|176761629-176762154| 248|+/-| 42|74| \mathrm{R}$ 100|P 0|S 100| \\
\hline 11 & chr3 $37579269-37579567$ & NA12878 $\mid$ AluYa4 $|37579379-37579434| 420|+/-| 20|25|$ R o|P 0|S 0| \\
\hline 12 & chr4 64409354-64409661 & NA12878|AluYc1 $|64409458-64409535| 371|+/-| 18|33| \mathrm{R} 0 \mid \mathrm{P}$ 0|S $0 \mid$ \\
\hline 13 & chr2 188871072-188871403 & NA12878|AluYb8 $|188871225-188871278| 375|+/+| 43|28| \mathrm{R}$ 0|P o|S $0 \mid$ \\
\hline 14 & chr5 84420069-84420377 & NA12878|AluYc1|84420215-84420245|385| \\
\hline
\end{tabular}

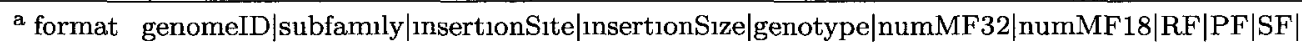

Table 3.5: Sample results of TIPs_OUT prediction.

\begin{tabular}{|c|c|c|c|c|}
\hline \multirow{2}{*}{ Genome(coverage) } & \multirow{2}{*}{ TE class } & \multirow{2}{*}{ TIPs_OUT } & \multicolumn{2}{|c|}{ Genotype } \\
\hline & & & $"+1+"$ & $"+1-"$ \\
\hline \multirow{2}{*}{ NA19240(36X) } & SVA & 65 & 39 & 26 \\
\hline & L1 & 224 & 96 & 128 \\
\hline \multirow{2}{*}{ (Nigerian child) } & HERV & 58 & 26 & 32 \\
\hline & Alu & 2379 & 842 & 1537 \\
\hline \multirow{2}{*}{ NA19238(24X) } & SVA & 25 & 8 & 17 \\
\hline & L1 & 145 & 52 & 93 \\
\hline \multirow{2}{*}{ (Nigerian father) } & HERV & 26 & 7 & 19 \\
\hline & $\mathrm{Alu}$ & 1865 & 250 & 1615 \\
\hline \multirow{2}{*}{ NA19239(28X) } & SVA & 45 & 25 & 20 \\
\hline & L1 & 211 & 87 & 124 \\
\hline \multirow{2}{*}{ (Nigerian mother) } & HERV & 57 & 29 & 28 \\
\hline & Alu & 2220 & 651 & 1569 \\
\hline \multirow{2}{*}{ NA12878(34X) } & SVA & 8 & 3 & 5 \\
\hline & L1 & 101 & 14 & 87 \\
\hline \multirow{2}{*}{ (Utah child) } & HERV & 33 & 15 & 18 \\
\hline & Alu & 799 & 57 & 742 \\
\hline \multirow{2}{*}{ NA12891(33X) } & SVA & 5 & 0 & 5 \\
\hline & L1 & 16 & 5 & 11 \\
\hline \multirow{2}{*}{ (Utah father) } & HERV & 12 & 7 & 5 \\
\hline & Alu & 174 & 13 & 161 \\
\hline \multirow{2}{*}{ NA12892(28X) } & SVA & 2 & 1 & 1 \\
\hline & L1 & 27 & 7 & 20 \\
\hline \multirow{2}{*}{ (Utah mother) } & HERV & 19 & 10 & 9 \\
\hline & Alu & 188 & 15 & 173 \\
\hline \multirow{4}{*}{$\begin{array}{l}\text { Total } \\
\text { (non-redundant list) }\end{array}$} & SVA & 91 & \multicolumn{2}{|c|}{ Grand total } \\
\hline & L1 & 456 & \multirow{3}{*}{\multicolumn{2}{|c|}{5569}} \\
\hline & HERV & 141 & & \\
\hline & Alu & 4881 & & \\
\hline
\end{tabular}

Table 3.6: Summary of TIPS_OUT predictions based on the six genomes. 


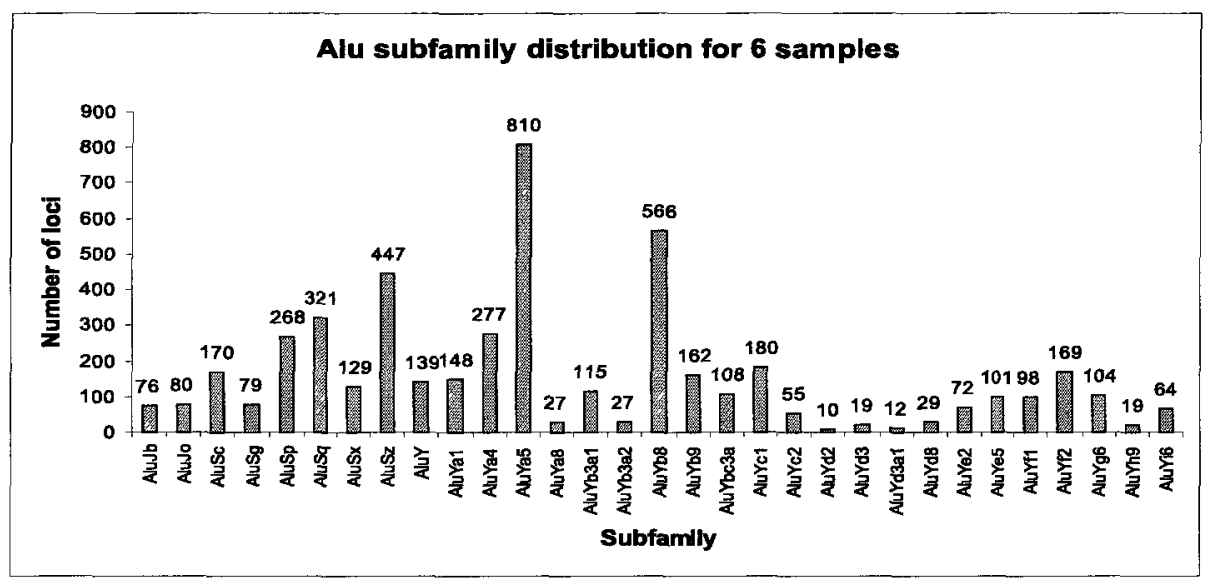

Figure 3.11: Alu subfamily distribution for 6 genomes.

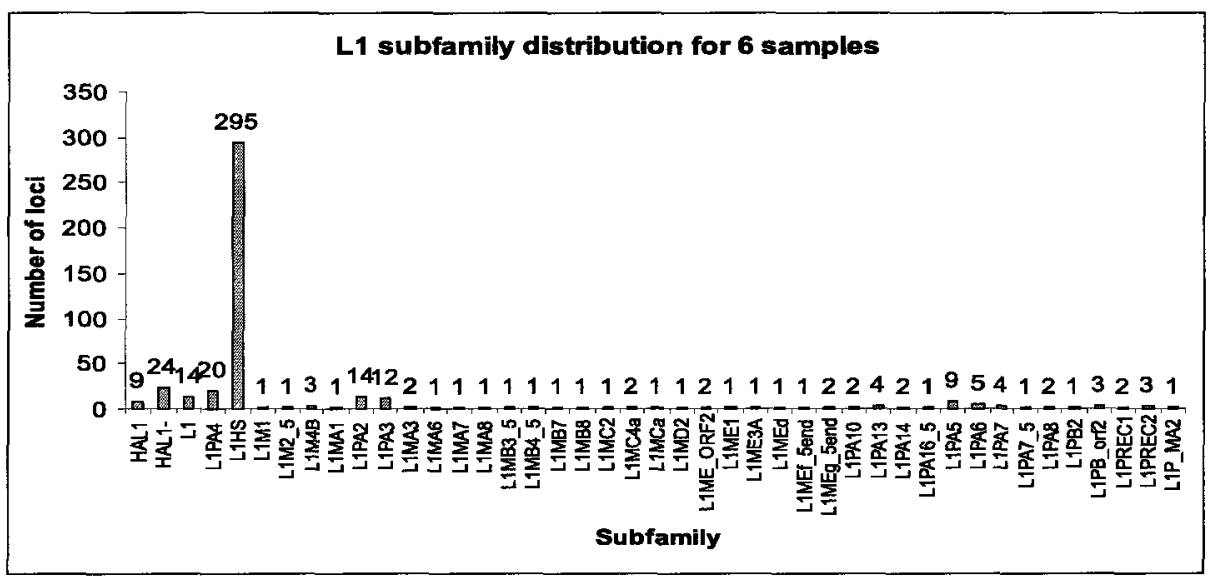

Figure 3.12: L1 subfamily distribution for 6 genomes.

TIP-finder identified a total of more than 5569 TIPs_OUT consisting of 4881, 456, 91, and 141 insertions from Alu, L1, SVA and HERV, respectively. Among the 3 types of TEs that are known to be active in the human genome, Alu has the largest number of TIPs_OUT as we could expect, reflecting its highest level of transposition and the large base number. It can be seen from the Alu subfamily distribution as shown in Figure 3.11 that AluYa5 and AluYb8 are the most active Alu subfamilies, which is consistent with the findings reported in [126]. Mills et al. [126] also reported that L1HS is the most active subfamily for L1. Our prediction shows that L1HS is the most active and accounts for more than $65 \%$ of the predicted L1s (see Figure 3.12). SVA_F which is the youngest SVA subfamily has the largest number in our prediction (see 
Figure 3.13). In addition to Alu, L1, and SVA, we identified 141 HERV TIPs_OUT, suggesting that they are still active contrasting to our current view. The HERV subfamily distribution for 6 genomes is shown in Figure 3.14. It is observed that HERVIP10FH-int which belongs to ERV1 family is the most active subfamily and LTR5_Hs which belong to ERVK family is the second most active subfamily.

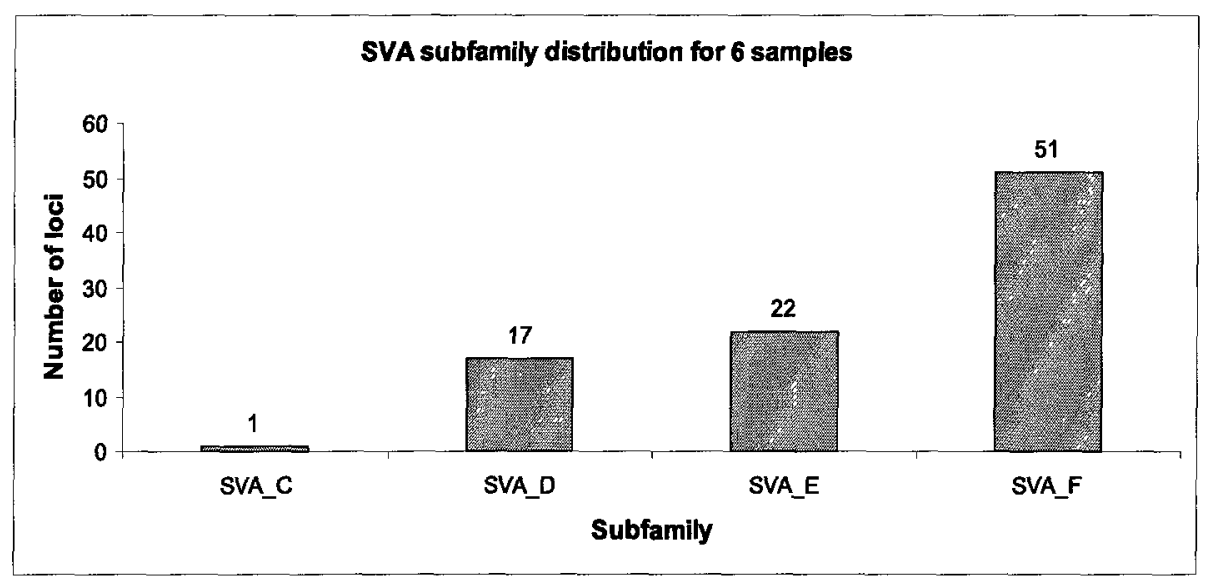

Figure 3.13: SVA subfamily distribution for 6 genomes.

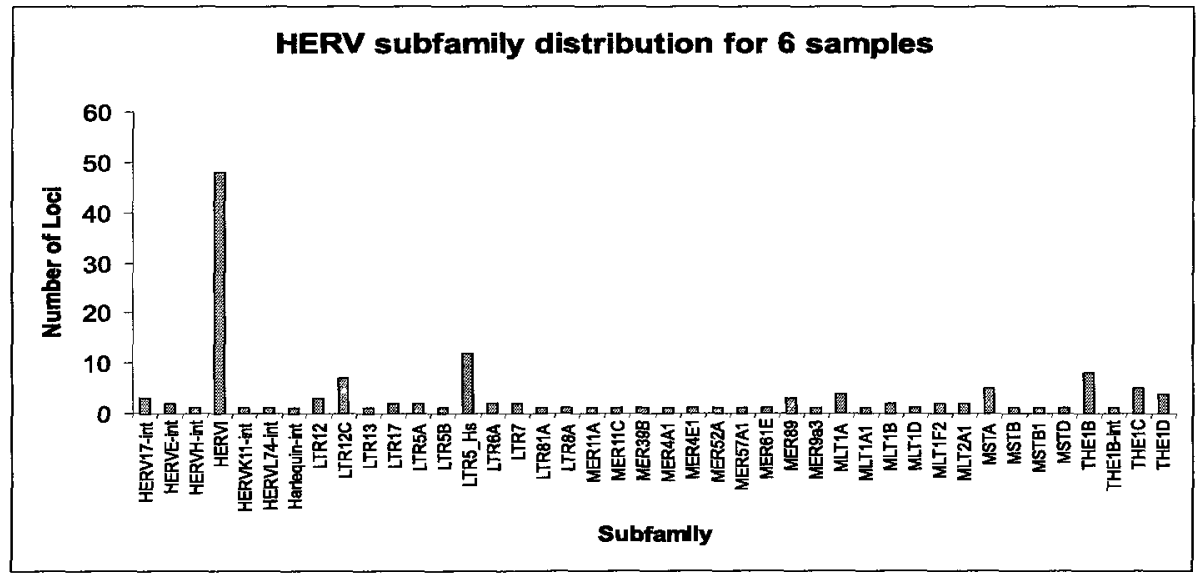

Figure 3.14: HERV subfamily distribution for 6 genomes.

As shown in Table 3.6, it is worth noting a few interesting patterns of TIPs_OUT. First, the numbers of TIPS_OUT from the two families are dramatically different, with the Nigerian family containing much more TIPs_OUT than the Utah Caucasian family (see Table 3.6). This is expected because the reference genome has mainly 
a Caucasian origin, thus is more similar to the Utah families than to the Nigerian family. The data suggest that a large number of new TE insertions have occurred in the genomes of the African populations after the migration of ancestors of current nonAfrican populations out of Africa. Second, the number of TIPs_OUT in heterozygous genotype ("+/-") is much higher than the homozygous genotype $("+/+$ ") for all TE types in most individuals. This is not only because these TE insertions follow the Hardy-Weinberg equilibrium, but also because TIPs_OUT represent more recent insertions and it takes time for them to reach the " $+/+$ " genotype in the populations. The fact that Alu has the highest ratio of "+/-" vs "+/+" among all TE types agrees with the fact that Alus have the largest subfamilies and are highly active TEs in the human genomes and thus have more recent insertions.

We compared the predicted TIPs_OUT with the 781 TIPs_OUT in dbRIP and found 453 (or $58 \%$ ) overlapping entries. This is a reasonable number considering the small number of genomes covered in this study. We also compared the predicted 4881 Alu TIPs_OUT with the 4343 Alus predicted based on 8 personal genomes by Hormozdiari [68], and found 1472 (or 33\%) overlapping. The percentage of overlap is relatively small, which is most likely due to the use of different personal genomes. Future work would apply our method to the same 8 personal genomes for more efficient comparison. Excluding the TIPs_OUT that overlap with those in dbRIP and those predicted by Hormozdiari, we found a total of 3984 novel TIPs_OUT including 3333 Alus, 426 L1s, 84 SVAs and 141 HERVs. This represents the most comprehensive analysis of retrotransposon insertion polymorphism. We also compared our predicted TIPs_OUT with those predicted by the 1000 genome project. Since a full list of TIPs predicted by the 1000 genomes browser is not available, we could not compare all our predicted TIPs_OUT with the 1000 genome project. We randomly chose 150 AluYa5 TIPs_OUT predicted by TIP-finder, and then compared them with the 1000 genomes browser and found 70\% overlap. Since the genomes used in this study are included in those reported in the 1000 genomes browser, we can expect that the results should mostly overlap. The fact that we found $30 \%$ more insertions for the six genomes indicates that our method perhaps provides a better sensitivity. 


\subsubsection{Experimental validation on predicted TIPs_OUT}

Some predicted TIPs_OUT results were validated by PCR experiments. As discussed before, in genotype "-/-", insertion is absent in both copies of the genome, and in genotypes " $+/+$ " and " $+/-$ ", insertion presents in both or only one copy of the genome, respectively. Table 3.7 shows the experimental validation results for the 14 Alu TIPs_OUT predicted by TIP-finder. For loci 8 and 10, all 6 genomes have genotype "-/-", suggesting no insertion is present in these two loci. For the other 12 loci, 11 insertions are present only in the Utah Caucasian family, and one insertion (loci 1) is present in both the Utah Caucasian and the Nigerian families. In total, 12 out of 14 (about 86\%) TIPs_OUT are confirmed to have insertions in at least one of six genomes by biological experiment. This further proved that our TIP-finder can identify TIPs_OUT with a high precision.

\begin{tabular}{|c|c|c|c|c|c|c|c|}
\hline Locus ID & Locus position & NA19238 & NA19239 & NA19240 & NA12891 & NA12892 & NA12878 \\
\hline 1 & $\operatorname{chr} 1855368680-55369412$ & $+/-$ & $+/-$ & $+/-$ & $+/-$ & $-/-$ & $-1-$ \\
\hline 2 & chr13 30789701-30790208 & $-1-$ & $-1-$ & $-1-$ & $+1-$ & $+1+$ & $+1+$ \\
\hline 3 & chr10 $98408229-98408714$ & $-1-$ & $-/-$ & $-1-$ & $-1-$ & $+/-$ & $+1-$ \\
\hline 4 & chr7 38444589-38445002 & $-1-$ & $-1-$ & $-1-$ & $-1-$ & $+1-$ & $+1-$ \\
\hline 5 & chr20 $37643378-37644453$ & $-/-$ & $-1-$ & $-1-$ & $-1-$ & $+1+$ & $+1-$ \\
\hline 6 & chr6 $32697176-32697966$ & $-1-$ & $-1-$ & $-1-$ & $-1-$ & $+/+$ & $+/+$ \\
\hline 7 & chr4 64409294-64409746 & $-1-$ & $-1-$ & $-1-$ & $+1-$ & $-1-$ & $+1-$ \\
\hline 8 & chr1 58977608-58978170 & $-1-$ & $-1-$ & $-1-$ & $-1-$ & $-1-$ & $-1-$ \\
\hline 9 & $\operatorname{chr} 8110170489-110171052$ & $-1-$ & $-1-$ & $-1-$ & $+1-$ & $-1-$ & $+1-$ \\
\hline 10 & $\operatorname{chrl} 176761255-176762231$ & $-/-$ & $-1-$ & $-1-$ & $-1-$ & $-/-$ & $-1-$ \\
\hline 11 & chr3 $37579150-37579784$ & $-/-$ & $-1-$ & $-/-$ & $-1-$ & $+/-$ & $+1-$ \\
\hline 12 & chr4 64409294-64409746 & $-/-$ & $-1-$ & $-/-$ & $-1-$ & $+/+$ & $+/-$ \\
\hline 13 & chr2 188870959-188871567 & $-1-$ & $-1-$ & $-1-$ & $+1-$ & $+1-$ & $+/+$ \\
\hline 14 & chr5 84420024-84420530 & $-1-$ & $-1-$ & $-1-$ & $+1-$ & $-/-$ & $+1-$ \\
\hline
\end{tabular}

Table 3.7: Summary of experimental validation on predicted TIPS_OUT.

\subsection{Discussions}

The personal genome data generated using the newer generation of sequencing technologies present an unprecedented grand opportunity for genetic study, particularly about the mechanisms of genetic variations, such as the structural variations, and their association with human diseases. The benefit mainly comes from the abundance of the sequence data and the affordability for accessing a large number of individuals representing a diverse human population. In the meantime, due to the nature of the sequence data being short in length with a high error rate, and the necessity of 
high level sequence redundancy to achieve sufficient coverage, it also creates many computational technical challenges to manage and analyze these data. These limitations require that the computational tools we develop to analyze these data must have efficiency for memory usage and speed in processing this type of data. For the analysis of structural variations, the identification of transposon insertion variations, particularly those that are absent in the reference genome or TIPs_OUT, represents the most difficult task mainly due to the repetitive nature of their sequences.

In developing TIP-finder, we utilize not only the special signatures of pairedend mapping associated with TIPs_OUT, but also annotation of transposons in the reference genome. More importantly, we incorporated the transposon biology into the establishment of a set of parameters for reducing the noise or false positive, a main issue associated with the analysis of TIPs. Our noise filtering parameters consider the minimal number of discordant pair reads characteristic of TIPs in relation to the genome coverage, the ratio of discordant paired-end reads vs the concordant reads, and the confidence level of PEM. Furthermore, we utilized a very unique data set, a simulated genome sequence containing known TIPs_OUT from dbRIP to train our algorithm by identifying the optimal combinatorial parameters in filtering the noise. We then used another set of data to test the algorithms. Enhanced by such a machinelearning approach, TIP-finder showed a high efficiency for the identification of TIPs. In addition to the prediction of insertion position, TIP-finder also provide prediction on subfamily, size, and genotype, which can be very valuable for the downstream experimental validation and analysis.

While we showed how TIP-finder uses flag information provided by MAQ [107] to identify TIPs_OUT, the method can also be applied to data aligned by other softwares such as BWA [106]. Although BWA does not provide MF32 flag, it provides flag information for discordant paired-end reads. In fact, if paired-end reads are discordant and mapped to different chromosomes, they can be considered as those reads with MF32 flag in MAQ alignment. The approach can also be applied to all discordant paired-end reads as initial input dataset instead of MF32 reads. Therefore, TIP-finder can easily be extended to use mapping information provided by other softwares to identify TIPs_OUT. 
In comparison with VariationHunter[69], TIP-finder is developed specifically to analyze the data generated by the 1000 genome project, which provides the alignment data generated by using either MAQ or BWA. Both MAQ and BWA map a read to the position where the sum of quality values of mismatched nucleotides is minimum. If a read can be mapped to several positions, they will randomly choose one position. As discussed in Section 3.2.8, in the method of VariationHunter, the minimum number of clusters are selected from the maximal valid clusters, which are generated based on the multiple mapping positions of all reads. Therefore, the multi-

ple mapping locations of the reads are essential input data for VariationHunter. Since the alignment data from MAQ or BWA do not have the information of other mapping locations, VariationHunter cannot be used to analyze the data generated by the 1000 genome project.

Since very stringent filtering criteria are used in this study, some real TIPs_OUT can be filtered out leading to false negatives. For example, at locus position chr1:2473007424730448 , biological experiment showed that an insertion is present in genome NA19238 with a genotype " $+/+$ ". However, this real TIPs_OUT was filtered out because its numMF32 (21) is smaller than the minimum numMF32 (29 for genotype " $+/+$ "). In fact, numMF32 is not only related to genome coverage, but also insertion size. The cutoff value of numMF32 was generated from our simulated data which contain the known TIPs_OUT. For the TIPS_OUT whose insertion sizes are smaller than those of simulated TIPs_OUT, they can be filtered out. The restriction on percentReliableMF32 excludes TIPs_OUT in the repetitive regions which tend to produce high level of false positives. However, this also leaves out the real TIPs_OUT in repetitive regions. Although the stringent filtering criteria improve the precision of our method, it may decrease the sensitivity of our method as well.

\subsection{Conclusions and future work}

SVs are a dominant and important type of genetic variation, and they contribute significantly to human diversity and disease susceptibility. The availability of rapidly increasing personal genome sequence data from the use of newer generations of sequencing technologies provides a very rich data source for surveying SVs in humans. 
Among all types of SVs, the identification of TIPs is more challenging due to the highly repetitive nature of transposon sequences.

We developed a computational method, TIP-finder, to identify TIPs through analysis of next generation personal genome data. TIP-finder predicts TIPs via a greedy algorithm based on the mapping patterns of paired-end reads associated with TIPs and other biological characteristics of transposons. Enhanced by a machine-learning approach, we tested the efficiency of TIP-finder using a simulated genome containing a set of known TIPs and were able to detect $\geq 88 \%$ of TIPs with a precision of $\geq 91 \%$. Despite the limited number of genomes analyzed, our data strongly suggest that the level of genetic variations from transposons is much higher than previously reported, and thus we can expect to identify much more of this types of genetic variation by analyzing more personal genome data.

We applied TIP-finder to six genomes subjected to deep sequencing using NGS and identified a total of 5569 TIPs, consisting of 4881, 456, 91, and 141 insertions from Alu, L1, SVA and HERV, respectively. There are 14 predicted Alu TIPs_OUT which were validated by PCR experiments and 12 of them are confirmed to have insertions in at least one of six genomes. This further proved that our TIP-finder can identify TIPs_OUT with a high precision. We demonstrate in this study that the rich personal genome sequence data generated using the next generation sequence technologies, albeit very challenging in managing and analyzing, provide a very valuable venue for studying genetic structural variations.

Future improvements for TIP-finder would include the support for sequence data generated by other types of NGS platforms, particularly those from the emerging new platforms, and more ideally the support of heterogeneous type of data. Future work also includes further characterization of each TIP locus at the sequence level via a localized de novo assembly. The length of current high throughput sequencing reads ranging from 35 to 250 base pairs, but the size of TIPs can be as long as a few thousand base pairs. In order to address this deficiency, a localized de novo assembly method will be developed to assemble the inserted transposon sequences. This method will involve an iterative process to identify or collect related reads and assemble the consensus sequence representing the TIP. At the end of each cycle, the 
consensus sequence is extended and used to identify new related reads for next round of assembly until no improvement of assembly can be made or no new reads can be found in existing assembly algorithm. Success in this method will not only further verify the candidacy of the TIPs, but also provide highly valuable information for the follow-up biological study. 


\section{Chapter 4}

\section{The cluster editing problem: implementation, improvement and application}

This chapter focuses on the cluster editing problem, implementation, improvement and application to predict protein complexes. A paper published by [37] is partly the basis for this chapter. The programming work was completed by both Dr. Yun Zhang in Professor Langston's group at University of Tennessee and myself. Dr. Zhang mainly contributed to the implementation of the FPT method for cluster editing, and I mainly contributed to the implementation of the greedy method and the LP method for cluster editing. For the improvement of the FPT-based method for cluster editing, Dr. Zhang and I contributed equally to this work. I also applied the cluster editing method to the PPI network to identify and predict protein complexes.

\subsection{Introduction}

A graph modification problem is about making a minimum number of modifications to a given graph so that a desired property of the graph can be met. The possible modifications include deletion and insertion. The cluster editing problem is one kind of graph modification problems, and it is defined as follows:

Input: An undirected graph $G=(V, E)$, and a non-negative integer $k$

Question: Is it possible to transform $G$ via at most $k$ insertion and deletion into a cluster graph?

A cluster graph is a graph that consists of a disjoint union of cliques. A clique is a graph where every two vertices have an edge between them. The cluster edit distance for a graph $G$ is the smallest value of $k$ for which cluster editing is possible. An example of the cluster editing problem is shown in Figures 4.1.

The cluster editing problem has been considered frequently in the literature since the 1980s. In 1986, Krivanek and Moravek showed that the cluster editing problem 

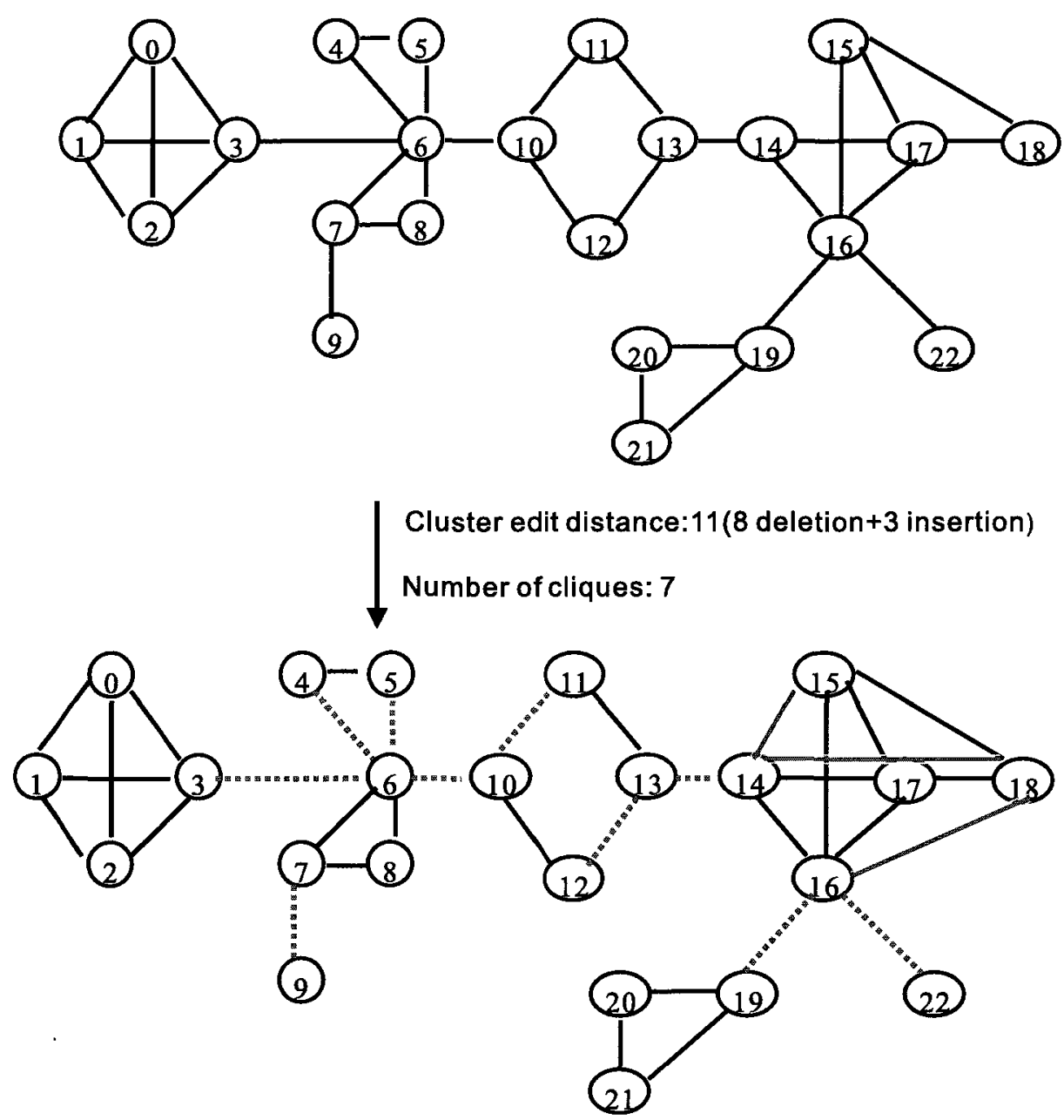

Figure 4.1: An example of the cluster editing problem. The dotted lines represent deleted edges and the grey lines represent added edges.

is non-deterministic polynomial-time hard (NP-hard) [98]. This problem was then rediscovered in the context of computational biology [159, 9]. Cai [20] proved that the cluster editing problem is fixed-parameter tractable. Many NP-hard problems can be formulated with a parameter $k$, so that when $k$ is fixed, the problems can be solved by polynomial-time algorithms. The theory of parameterized complexity was developed by Downey and Fellows [40], as an alternative to deal with such NPhard problems. A problem is fixed-parameter tractable if its input can be partitioned into a main part of size $n$ with an integer parameter $k$ so that there is an algorithm 
that solves the problem in time $O\left(n^{c} \cdot f(k)\right)$, where $f$ is a computable function only depending on $k$ and $c$ is a fixed constant [40]. The algorithm is called fixed-parameter algorithm. A kernel is an instance of the problem smaller than the input, such that the problem has a solution on the input if and only if it has a solution on the kernel. It is well known that a problem is fixed-parameter tractable if and only if a kernel of size $g(k)$ can be computed from the input in polynomial time, for a computable function only depending on $k[40,127]$.

In this study, we implemented a fixed-parameter tractability (FPT)-based method for cluster editing using the algorithm developed by Guo and coworkers[56, 57]. This was the first implementation of an FPT-based method for cluster editing. In order to evaluate the effectiveness of the FPT-based approach, we also implemented a previously well known approximation method based on linear programming (LP) [25] as well as a greedy approach for cluster editing. Our experiments showed that the best performance for cluster editing was obtained by using the refined branching method in [57] together with interleaving (re-kernelization). Our experiments also showed that the refined branching method in [57] was vastly superior to the basic branching method, which is not obvious because the refined branching method is considerably more complicated and incurs larger constant factors. We also demonstrated that, in practice, branching with interleaving was indeed faster than branching without interleaving.

After implementation of the approach described in $[56,57]$, we then developed a number of algorithms to improve the performance of the FPT-based method for cluster editing. These include a greedy method for choosing branching orders, a dynamically updated matrix for neighbor information, an LP method for choosing branching orders, and a parallel FPT-based method. With the new optimization methods, the performance of the FPT-based method was greatly improved. We also applied our cluster editing method to the protein-protein interaction (PPI) networks of yeast to identify and predict protein complexes. The comparison of the protein clusters found by our method with known yeast protein complexes showed that our method has potential to identify known protein complexes and also to predict some new protein interaction associated with in the complexes. 


\subsection{State of the art in algorithms for the cluster editing problem}

The cluster editing problem is NP-hard, and it has been studied with respect to approximation and fixed-parameter tractability. The following section summarizes approximation algorithms and fixed-parameter algorithms for the cluster editing problem.

\subsubsection{Approximation algorithms}

Approximation algorithms are used to find approximation solutions to optimization problems, usually when the problems are NP-hard. The quality of an approximation algorithm is measured by the approximation factor, which is the approximation of the optimum solution. Since the cluster editing problem is NP-hard, a number of research groups developed approximation algorithms to solve this problem as follows.

Bansal and coworkers [9] initiated the research on correlation clustering which is motivated by applications in machine learning. The correlation clustering problem is considered as follows: given a fully-connected graph $G=(V, E)$ with each edge labeled either "+" (similar) or "-" (different), partition the vertices into clusters that agrees as much as possible with the edge labels. That is, finding a clustering that maximizes the number of "+" edges within clusters plus the number of "-" edges between clusters. Similarly, minimizing disagreements is to minimize the number of "-" edges within clusters plus the number of "+" edges between clusters. In fact, minimizing disagreements is identical to cluster editing, and cluster editing is a special case of the correlation clustering problem. Unlike most clustering formulations, in the correlation clustering problem, the number of clusters is no longer a parameter of the input. The optimal clustering might have a different number of clusters, and the ideal number of clusters depends on the edge labels.

Bansal and coworkers[9] gave a polynomial time approximation scheme (PTAS) for the problem of maximizing agreements. For the problem of minimizing disagreement, they first provided a simple algorithm that is at most three times worse than the best clustering containing two clusters. They then presented a constant factor approximation (see Table 4.1). The constant is a rather large one (about 17433), but it demonstrated that a constant approximation can be achieved. Their solutions 
for both minimizing disagreement and maximizing agreements are based on the unweighted complete graphs. For weighted complete graphs, where each edge is assigned fractional value $w_{\imath \jmath}^{+}$or $w_{\imath \jmath}^{-}$rather than a discrete "+" or "-" label, Bansal and coworkers proved that there exists an algorithm that can achieve an approximation ratio of $(2 p+1)$ under a linear cost function, where $p$ is the approximation ratio for unweighted complete graphs. As shown in Table 4.1, Bansal and coworkers also proved that minimizing disagreement for the weighted general graphs is approximable(APX)hard, thus admitting there is no PTAS for the weighted general graph problem. They treated the problem of finding approximation algorithms for general graphs as open questions, where not all edges are labelled either "+" or "-", meaning that some edges do not have information. They also treated the problem of finding hardness of approximation for both unweighted complete graph and general graph as open questions.

\begin{tabular}{|c|c|c|c|}
\hline Type of Graph & Approximation & Hardness of approximation & Equivalence \\
\hline $\begin{array}{c}\text { Unweighted } \\
\text { complete }\end{array}$ & $c \in O(1)[9]$ & Open question [9] & \\
graph & $c=4[25]$ & APX-hard [25] & \\
\hline $\begin{array}{c}\text { Weighted } \\
\text { complete }\end{array}$ & $9=3[3]$ & & \\
graph & $3[54]$ & APX-hard [9] & \\
\hline Unweighted & Open question [9] & & \\
general graph & $O(\operatorname{logn})[38,45]$ & Open question [9] & Unweighted \\
\hline Weighted & Open question [9] & APX-hard [38, 45] & multicut [38, 45] \\
general graph & O(logn) [38, 45, 25] & & Weighted \\
multicut [38, 45]
\end{tabular}

Table 4.1: The approximation results on the problem of minimizing disagreements.

The open questions posed by Bansal and coworkers motivated a number of groups $[38,45,25]$ to work on the minimizing disagreements problem simultaneously, and several better approximations and lower bounds were developed for general graphs (see Table 4.1). Demaine and Immorlica [38] presented a factor $O(\log n)$ algorithm for general graphs, based on a linear-programming, rounding and the region-growing technique. Independently of Demaine and Immorlica's work, Emanuel and Fiat [45] provided an $O(\log n)$ approximation algorithm for minimizing disagreements on weighted and unweighted general graphs. They also proved that the problem of minimizing 
disagreements is APX-hard for the unweighted general graphs, and showed that the minimizing disagreements problem is equivalent to the minimum multicuts problem. The approximation for minimizing disagreements in the unweighted complete graphs was improved to a factor of 4 by Chairkar and coworkers [25]. They also proved that the minimizing disagreements problem is APX-hard for the unweighted complete graphs (see Table 4.1). According to Bansal and coworkers[9], an approximation ratio of $(2 p+1)$ can be achieved for weighted complete graphs where $p$ is the approximation ratio for unweighted complete graphs. Thus, an approximation ratio of 9 can be obtained for weighted complete graphs by combining the results of [9] and [25] $(p=4)$.

In 2005, Gionis et al. [54] presented an approximation algorithm called the Balls algorithm for correlation clustering on weighted completes graphs, and proved that the cost of the BALLS algorithm is at most 3 times that of the optimal clustering when the edge weights satisfy the probability and triangle inequality constraints. Probability constraints have the form $w_{\imath \jmath}^{+}+w_{\imath \jmath}^{-}=1$, and triangle inequality constraints have the form: for all $i, j, k, w_{\imath \jmath}^{+}+w_{\jmath k}^{+}+w_{\imath k}^{-} \leq 2$.

In the same year, Alion et al. [3] developed an improved approximation algorithm called CC-PIVOT algorithm and proved it is a randomized expected 3-approximation algorithm for correlation clustering on unweighted complete graphs, which improved the results demonstrated in [25]. They also applied the algorithm on weighted complete graphs, and proved when the edge weights satisfy the probability and triangle inequality constraints, its approximation factor is 2 , which improved the results demonstrated in [54].

\subsubsection{Fixed-parameter algorithms for cluster editing}

In 1996, Cai [20] reported the research on the fixed-parameter tractability of a graph modification problem. The problem is to decide whether a graph can be made into a graph with a specified hereditary property by deleting at most $i$ vertices, at most $j$ edges, and adding at most $k$ edges, where $i, j, k$ are fixed integers. It is shown that the problem is fixed-parameter tractable whenever the hereditary property can be characterized by a finite set of forbidden induced subgraphs. The cluster editing 
problem is fixed-parameter tractable, and its forbidden set is a $P_{3}$, which is a graph containing three vertices and two edges. That is, $P_{3}$ cannot be contained in the final graph of cluster editing. Cai developed an $O\left(3^{k} \cdot|n|^{4}\right)$ algorithm for cluster editing. The running time of the algorithm is mainly determined by the size of the resulting search tree. Gramm and coworkers [56] introduced a straightforward branching strategy that leads to a search tree of size $O\left(3^{k}\right)$ by destroying all occurrences of an induced $P^{3}$. This result was further improved in the same study by a more sophisticated branching strategy to a search tree of size $O\left(2.27^{k}\right)[57,56]$. The algorithm consists of two parts: it first builds a problem kernel with $O\left(k^{2}\right)$ vertices and $O\left(k^{3}\right)$ edges in $O\left(n^{3}\right)$ time; and then applies a bounded search tree in $O\left(2.27^{k}\right)$. The time complexity of building the problem kernel with $O\left(k^{2}\right)$ vertices was further improved to $O(n+m)$ ( $m$ is the number of edges) by Protti et al.[139, 140] based on modular decomposition techniques. The time complexity of the search tree strategy was also further improved to $O\left(1.92^{k}\right)$ based on automated search tree generation and analysis [55].

In recent years, two research groups[49, 58] presented polynomial-time kernelization algorithms for cluster editing that lead to linear size kernels. Fellows et al. [49] reported how a crown-type structural reduction rule can be used to obtain a $6 k$ kernelization bound. Guo [58] presented an $O\left(n^{3}\right)$ time algorithm that achieves a problem kernel of size at most $6 k$. This bound is then further improved to $4 k$ via an $O\left(n m^{2}\right)$ time kernelization algorithm in the same study.

The above fixed-parameter algorithms were studied for an ordinary graph, where each edge has information on whether it is similar or different. For general graphs where some edges have missing information, the cluster editing problem has previously been studied only with respect to approximation. Bodlaender et al. [16] recently reported a fixed-parameter algorithm for the cluster editing problem for general graphs. They proved that the correlation clustering problem is fixed-parameter tractable on general graphs when parameterized by $(k, r)$, where $k$ is the editing cost and $r$ is the minimum number of vertices required to cover the undecided edges. In particular, they showed a polynomial-time reduction to a problem kernel on $O\left(k^{2}+r\right)$ vertices. 


\subsubsection{Review of the algorithms implemented by this study}

In this study, we implemented the LP-based approximation algorithm described in $[25]$ and the fixed-parameter algorithms described in $[57,56,58]$ as reviewed below.

\subsubsection{The approximation algorithm}

The approximation algorithm (see Algorithm 11) developed by Chairkar et al. [25] is based on a natural LP relaxation. In LP, for each pair of vertices $i$ and $j$, a partition into clusters can be represented with a binary variable $x_{\imath \jmath}$, where $x_{\imath \jmath}=0$ if $i$ and $j$ are in the same cluster, and $x_{\imath \jmath}=1$ if they are in different clusters. The integer constraints can be relaxed to allow rational values for $x_{\imath \jmath}$, that is, $0 \leq x_{\imath \jmath} \leq 1$. For each triple of vertices $i, j$ and $k$, the triangle inequality $x_{\imath k} \leq x_{\imath \jmath}+x_{\jmath k}$ holds because if $x_{\imath \jmath}=0$ and $x_{\jmath k}=0$ then $x_{\imath k}=0$. The objective is to minimize the number of edge edits: the number of edges $(i, j) \in E$ for which $x_{\imath \jmath}=1$ and the number of pairs of vertices that are not adjacent $(i, j) \notin E$ for which $x_{\imath \jmath}=0$. That is, to minimize the number of edges $(i, j) \in E$ in different clusters and the number of edges $(i, j) \notin E$ in the same cluster.

After the LP model is solved, the AlgComplete algorithm is used to partition the graph into different clusters based on the variable $x_{\imath \jmath}$, which represents the distance between $i$ and $j$. Vertices that are close are placed in the same cluster, and vertices that are far are placed in different clusters. Chairkar and coworkers [25] proved that the AlgComplete algorithm achieves a factor of 4 approximation for cluster editing.

Algorithm 11 The AlgComplete algorithm

(1) Let $S=V$ and repeat the following steps until $S$ is empty.

(2) Select a vertex $u$ arbitrarily from $S$.

(3) Let $T$ be the set of vertices whose distance from $u$ is no greater than $1 / 2$, except $u$ itself: $B_{x}(u, 1 / 2)-\{u\}$.

(4) If the average distance of the vertices in $T$ from $u$ is not less than $1 / 4$, then make $C=\{u\}$ a singleton cluster and jump to step 6 .

(5) If the average distance is less than $1 / 4$, then make $C=\{u\} \cup T$ a cluster.

(6) Let $S=S-C$ and jump to step 2 (the start of the loop).

- End of Algorithm - 


\subsubsection{The fixed-parameter algorithm}

The fixed-parameter algorithm developed by Guo and coworkers $[57,56]$ consists of two steps: kernelization and branching. Two kenelization rules have been reported and a problem kernel for cluster editing after kenelization was proved to consist of at most $\left(k^{2}+4 k\right)$ vertices and at most $\left(k^{3}+4 k^{2}+k\right)$ edges. Two kernelization rules can be executed in $O\left(n^{3}\right)$. For branching, there are two search tree algorithms: basic branching strategy and refined branching strategy. The basic branching strategy leads to a search tree of size $O\left(3^{k}\right)$, and the refined search tree strategy leads to an improved search tree of size $O\left(2.27^{k}\right)$.

Two kernelization rules for the cluster editing problem have been described. The first rule is based on the neighborhood of every pair of vertices $u, v \in V$.

Rule 1:

(1) If $u$ and $v$ have more than $k$ common neighbors, then $(u, v)$ has to belong to $E$; if $(u, v) \notin E$, it should be added to $E$.

(2) If $u$ and $v$ have more than $k$ non-common neighbors, then $(u, v)$ cannot belong to $E$; if $(u, v) \in E$, it should be deleted.

(3) If $u$ and $v$ have both more than $k$ common and more than $k$ non-common neighbors, then the given instance has no size $k$ solution.

Rule 2: delete the connected components that are cliques from the graph.

The basic bounded search tree method for cluster editing is based on the observation that an induced path $P_{3}$, a path with three vertices and two edges, is forbidden for a graph consisting of disjoint cliques. Given a graph, considering any induced $P_{3}=\{u, v, w\}$ with edges $(u, v)$ and $(u, w)$, there are three cases of branching: delete edge $(u, v)$, delete edge $(u, w)$, or add edge $(v, w)$. For either case, the parameter $k$ is decreased by one. For basic branching, this leads to the search tree size of $O\left(3^{k}\right)$ where a resulting graph with disjoint cliques is found for $k$ edits.

There are two termination conditions:

(1) At each branch node, if the parameter $k$ goes down to be non-positive, no solution of size $\leq k$ exists on that branch.

(2) No $P_{3}$ can be found in the graph 
If no solution is found on all branches, then we conclude that no solution of size $k$ exists for the given graph. If and only if condition 2 is met and the value of $k$ is still positive, we conclude that there is a solution.

The basic bounded tree search method can be improved by making a case distinction of $P_{3}$ with three cases and giving each case a branching rule. Consider a $P_{3}=\{u, v, w\}$ with edges $(u, v)$ and $(u, w)$. There are three cases based on the neighborhood of $u, v$ and $w$ :

(1) $v$ and $w$ do not share a common neighbor other than $u$;

(2) $v$ and $w$ have a common neighbor $x$ other than $u$, and $x$ is adjacent to $u$;

(3) $v$ and $w$ have a common neighbor $x$ other than $u$, but $x$ is not adjacent to $u$.

For each pair of vertices, an Annotation Mapping to facilitate the branching rules is denoted as follows:

(1) Permanent: $(u, v) \in E$ and $(u, v)$ cannot be deleted

(2) Forbidden: $(u, v) \notin E$ and $(u, v)$ cannot be inserted

(3) None: if no information available, and it can be edited

For every three vertices $u, v, w \in V$, there are two Annotation Rules:

(1) If $(u, v)$ and $(u, w)$ are permanent, then $(v, w)$ has to be permanent. If $(v, w) \notin E$, $(v, w)$ has to be added to $E$ and be denoted as permanent.

(2) If $(u, v)$ is permanent and $(\mathrm{u}, \mathrm{w})$ is forbidden, then $(v, w)$ has to be forbidden. if $(v, w) i s \in E$, then it has to be deleted and denoted as forbidden.

\section{Algorithm 12 Refined Branching Strategy}

Given a graph $G=(V, E)$ and parameter $k$, consider a $P_{3}=\{u, v, w\}$ with edges $(u, v)$ and $(u, w)$. The refined branching strategy using the above annotation mapping works as follows.

(1) If $v$ and $w$ do not share a common neighbor other than $u$ (case $C 1$ ), then branch with

(a) $(G \backslash\{(u, v)\}, k-1)$, and

(b) $(G \backslash\{(u, w)\}, k-1)$.

(2) If $v$ and $w$ have a common neighbor $x \neq u$ and $(u, x) \in E$ (case $C 2$ ), then branch with five subcases (see Figures 4.2): 
(c) $(G \cup\{(v, w)\}, k-1)$;

(d) Set $(v, w)$ to forbidden, and branch with $(G \backslash\{(u, v),(v, x)\}, k-2)$;

(e) Set $(v, w)$ to forbidden, $(v, x)$ to permanent, and branch with $(G \backslash\{(u, v)$, $(u, x),(w, x)\}, k-3)$

(f) Set $(v, w)$ to forbidden, and branch with $(G \backslash\{(u, w),(w, x)\}, k-2)$;

(g) Set $(v, w)$ to forbidden, $(w, x)$ to permanent, and branch with $(G \backslash\{(u, w)$, $(u, x),(v, x)\}, k-3)$.

(3) If $v$ and $w$ have a common neighbor $x \neq u$ and $(u, x) \notin E$ (case $C 3$ ), then branch with five subcases (see Figures 4.3):

(h) $(G \backslash\{(u, v)\}, k-1)$;

(i) Set $(u, v)$ to permanent, $(v, w)$ to forbidden, and branch with $(G \backslash\{(u, w)$, $(v, x)\}, k-2)$;

(j) Set $(u, v)$ to permanent, $(v, w)$ to forbidden, $(\mathrm{v}, \mathrm{x})$ to permanent, and branch with $(G \cup\{(u, x)\} \backslash\{(u, w),(w, x)\}, k-3)$;

(k) Set $(u, v)$ and $(u, w)$ to permanent, and branch with $(G \cup\{(v, w)\} \backslash\{(w, x)$, $(v, x)\}, k-3)$;

(l) Set $(u, v)$ and $(u, w)$ to permanent, and branch with $(G \cup\{(v, w),(u, x)\}$, $k-2)$.

- End of Algorithm -

Initially, all vertex pairs are set to "none". When an edge is added it is set to "permanent", and when an edge is deleted it is set to "forbidden". The algorithm also stops when the parameter $k$ reaches 0 or below or when the graph $G$ contains no induced $P_{3}$. The search tree size for the refined branching strategy is $O\left(2.27^{k}\right)$.

\subsubsection{The linear kernelization}

Guo [58] developed a new data reduction rule to improve a kernel with a quadratic number of vertices to one with a linear number of vertices. In this work, Guo introduced a new concept known as critical clique. A critical clique of a graph $G$ is a clique $K$ where all the vertices of $K$ have the same set of neighbors in $V \backslash K$, and 


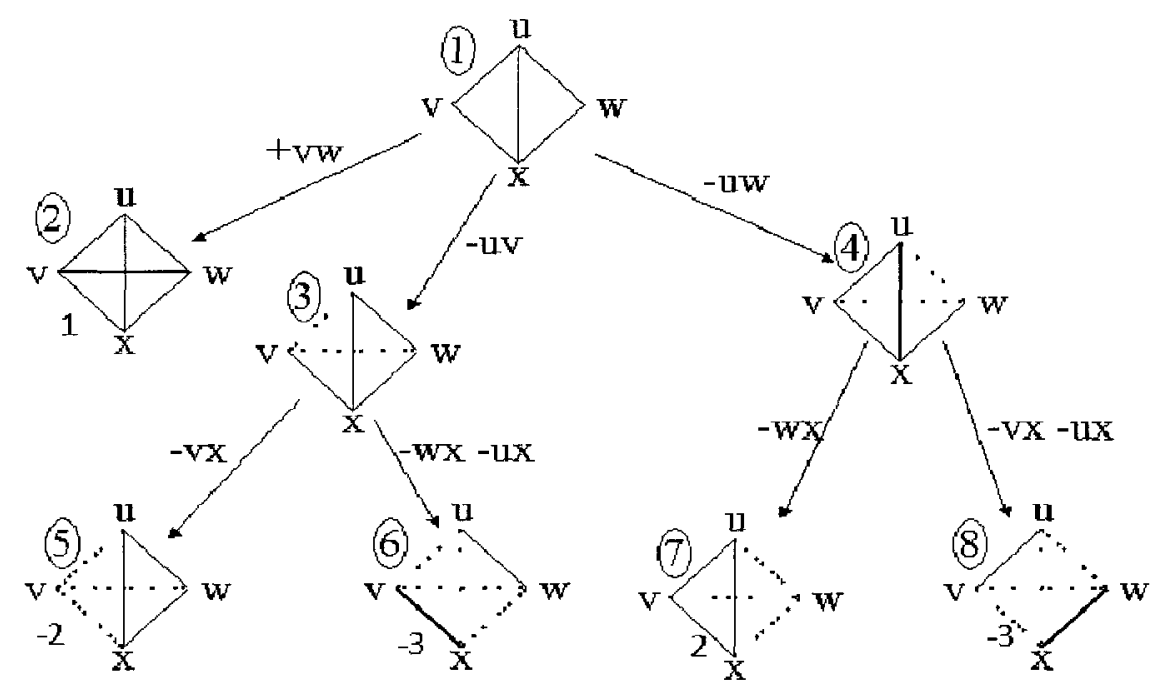

Figure 4.2: The branching rule for case C2 [57]. The bold lines denote edges annotated as permanent, the dashed lines denote edges annotated as forbidden.

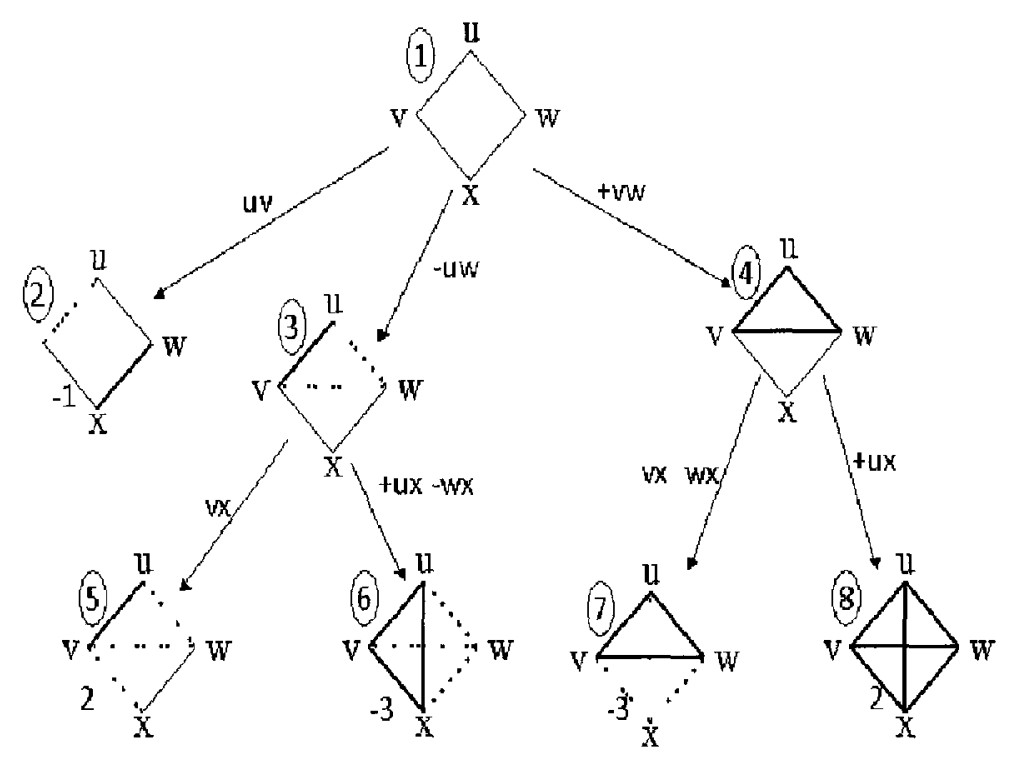

Figure 4.3: The branching rule for case C3 [57].

$K$ is maximal under this property. The critical clique graph is a graph with critical cliques as nodes, and two nodes are connected if and only if the corresponding critical cliques together form a larger clique (see Figure 4.4). According to Hsu and Ma [70], 
the critical clique graph can be constructed in $O(m+n)$, where $n$ is the number of vertices and $m$ is the number of edges.

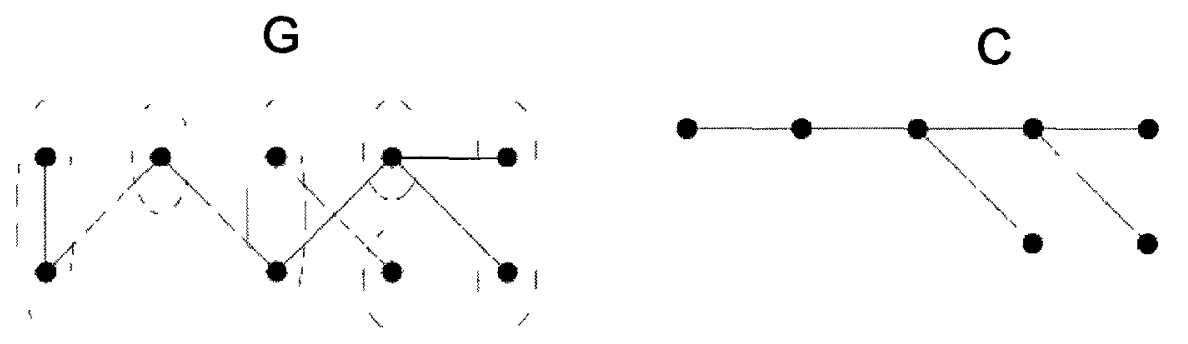

Figure 4.4: A graph $G$ and its critical clique graph $C$ [58]. Ovals represent critical cliques of $G$.

Guo described the basic idea behind introducing critical cliques as follows: given a solution with at most $k$ edge modifications for a graph $G=(V, E)$, at most $2 k$ vertices are "affected" by these edge modifications, i.e. two endpoints for each edited edge. Thus, in order to give a size bound on $V$ depending only on $k$, it needs to upperbound the size of the unaffected vertices. In the final cluster graph, the unaffected vertices contained in one clique must form a critical clique in the original graph $G$. Therefore, it seems easier to derive kernelization rules working on the critical clique graph than to derive rules directly working on the input graph.

Two kernelization rules for both input graph $G$ and critical clique graph $C$ are described in the following. Note that $V(K)$ denotes the set of vertices contained in a node $K$ in graph $C, N_{C}(K)\left(N_{c}[K]\right)$ denotes the open (closed) neighborhood of $K$, and $N_{c}^{2}(K)$ denotes the set of nodes in $C$ which have a distance of exactly 2 to $K$.

Rule 1: Remove all isolated critical cliques $K$ from $C$ and remove $V(K)$ from $G$. Rule 2: If, for a node $K$ in $C$, it holds $|V(K)|>\left|\bigcup_{K^{\prime} \in N_{c}(K)} V\left(K^{\prime}\right)\right|+\left|\bigcup_{K^{\prime} \in N_{c}^{2}(K)} V\left(K^{\prime}\right)\right|$, then remove nodes $K$ and $N_{C}(K)$ from $C$ and remove the vertices in $\bigcup_{K^{\prime} \in N_{c}[K]} V\left(K^{\prime}\right)$ from $G$. Accordingly, decrease parameter $k$ by the sum of the number of edges needed to transform subgraph $G\left[\bigcup_{K^{\prime} \in N_{c}(K)} V\left(K^{\prime}\right)\right]$ into a complete graph and the number of edges in $G$ between the vertices in $\bigcup_{K^{\prime} \in N_{c}(K)} V\left(K^{\prime}\right)$ and the vertices in $\bigcup_{K^{\prime} \in N_{c}^{2}(K)} V\left(K^{\prime}\right)$. If $k<0$, then the given instance has no solution.

Compared with Guo's previous kernelization rules in [57, 56], Rule 2 is a new reduction rule. Guo proved that the above new kernelization rules lead to a $6 \mathrm{~K}$ vertex kernel. Rule 1 and Rule 2 can be carried out in $O(m+n)$ and $O\left(n^{3}\right)$ time, 
respectively.

\subsection{Methods}

In order to evaluate the effectiveness of a fixed-parameter approach for cluster editing, we need to establish a baseline to which we can compare our implementation. As discussed previously, one well known method described in [25] is based on LP. An alternative is to use a greedy approach for cluster editing. Needless to say, both methods only provide an approximation of the edit distance. This section will present how we implement the three methods for cluster editing.

\subsubsection{A simple greedy heuristic}

Given a graph $G$, all possible edges are considered for an edge insertion, and only the edges in $G$ are considered for an edge deletion. A cost for each insertion/deletion is calculated as follows: For an edge $e=(i, j)$ the common neighborhood is defined as the set of common neighbors of $i$ and $j$, and the non-common neighborhood is defined as the set of non-common neighbors of $i$ and $j$. For an insertion of an edge $e=(i, j)$, the cost is defined as the number of edge insertions required to transform the common and non-common neighborhood of $e$ into a clique. For a deletion of an edge $e=(i, j)$, the cost is defined as the number of edge deletions required to disconnect the common neighborhood of $e$. The edit operation is selected with the smallest cost and the program iterates until a graph of disjoint cliques is obtained. To implement this greedy method, we initially label every pair of vertices as unmarked. For each unmarked pair of vertices $i$ and $j$, the smaller of the cost of having an edge between them and the cost of not having an edge between them is chosen as the cost of $i$ and $j$. We select the pair with least cost, perform the edits associated, and mark the pair. This is repeated until all pairs are marked, which will give a set of connected components. The edit distance is calculated as the number of edge editions to get the set of connected components plus the number of edge editions to transform those connected components into cliques. 


\subsubsection{An LP approach}

Given a graph, we first build an LP model by setting the objective function and constraints for every pair of vertices and every triple of vertices as described in Section 4.2.1. After the LP model is built, an LP C library (lp_solve version 5.5) is used to solve the LP model and generate the value for every variable $x_{\imath \jmath}$. Finally, the graph is partitioned into clusters based on the variables $x_{\imath \jmath}$ in the following way: two vertices $\imath$ and $\jmath$ are put into the same cluster if $x_{\imath \jmath} \leq 0.5$. The edit distance is calculated as the summation of the number of edges that are needed to be added, which do not exist but the two endpoints are in the same cluster, and the number of edges that are needed to be deleted, which exist but the two endpoints are in different clusters.

Algorithm 13 Solving the Cluster Edit Problem Via a LP based approach [25]

(1) build a LP model:

(a) objective function:

$$
\text { to minimize } \sum_{(\imath, \jmath) \in E} x_{\imath \jmath}+\sum_{(\imath, \jmath) \notin E}\left(1-x_{\imath \jmath}\right)
$$

(b) constraints:

for each pair of vertices $i$ and $\jmath: 0 \leq x_{\imath \jmath} \leq 1$

for each triple of vertices $i, j$ and $k, x_{2 k} \leq x_{2 \jmath}+x_{\jmath k}$

(2) solve LP model and get value for $x_{\imath \jmath}$

(3) for each pair of vertices $i$ and $j$ :

if $x_{\imath \jmath} \leq 0.5, i$ and $\jmath$ are in the same cluster

otherwise, $\imath$ and $j$ are in different clusters

- End of Algorithm -

\subsubsection{An FPT-based approach}

The following presents an outline of our adaptation of the algorithm described in $[57,56]$ that we used to obtain the first practical implementation for exact cluster edit distance computation. 
Algorithm 14 Solving the Cluster Edit Problem Via a Fixed-Parameter Tractability Approach

(1) Extract bioconnected components. Our motivation is to eliminate sparse parts of the input.

(2) Bound the search space for $k$ :

(a) Let $k_{L P}$ be the edit distance determined by the linear programming method described in Section 13.

(b) The search interval for the true edit distance $k$ is $\left[k_{L P} / 4, k_{L P}\right]$.

(3) For increasing $k$, starting with $k_{L P} / 4$ :

(a) Execute the kernelization method described in Section 4.2.3.2

(b) Execute either the basic or the refined bounded tree search method described in Section 4.2.3.2.

(c) Use "interleaving": at each branch node in the bounded tree search, execute again the kernelization method from Step 3a.

_ End of Algorithm _

For both basic and refined bounded tree search methods, we apply the kernelization method at each branch node. Both methods are implemented as recursive functions.

\subsubsection{An approach to extract biconnected components}

A biconnected component is a maximal biconnected subgraph. A biconnected graph is a graph that remains connected if any single vertex is deleted. In other word, a biconnected graph has no cut vertex. A cut vertex is a vertex of a graph such that the graph was connected before the removal of the vertex, and it will be disconnected afterwards.

We use the algorithm described in [82] to extract biconnected components. A depth-first search (DFS) is used in a given graph $(V, E)$, where the traversed vertices are labeled with consecutive numbers from 1 to $n=|V|$. There are two kinds of edges: one is the tree edge that leads to unlabeled vertices, and the other is the backward edge leading to vertices that were already discovered and labeled in a former step. 
Each vertex $v$ has a lower value (low[v]). It is the smallest label of any vertex that is reachable by tree edges followed by no more than one backward edge. When a new vertex is discovered by the DFS, its lower value is initialized by its own number. If a backward edge $(v, w)$ is discovered, low[v] should be updated to be the minimum of its current value and the label of $w$ (num[w]). When returning from a child $w$ from a tree edge $(v, w)$, low $[\mathrm{v}]$ should be updated to be the minimum of its current value and low $[\mathrm{w}]$.

To detect the cut vertices of the graph, the rules described in [82] are used as follows: A vertex $v$ is a cut-vertex if and only if one of the following conditions holds:

(1) if $v$ is the root of the DFS tree and is incident to at least 2 DFS tree edges

(2) if $v$ is not the root, but there is a child $w$ of $\mathrm{v}$ such that low $[\mathrm{w}] \geq \operatorname{num}[\mathrm{v}]$

To find the biconnected components, every new edge is put on a stack. After returning from a recursive call for a child $w$ of $v$, if low $[\mathrm{w}] \geq \operatorname{num}[\mathrm{v}]$, the edges on top of stack including edge $(v, w)$ form the next component.

\subsubsection{An approach to convert a graph to a critical clique graph}

We first developed a method to find a critical clique (see Algorithm 15) as follows: randomly choose a vertex $v$ and assign it to a critical clique $C_{S}$. For any vertex $u$, if it is connected to all vertices in $C_{S}$, and also $u$ and $C_{S}$ have the same neighbors, then $u$ belongs to $C_{S}$. The process is repeated until no such $u$ exists.

Algorithm 15 Approach to find a critical clique

(1) take any vertex $v$

(2) $C_{S}=\{v\}$

(3) select a vertex $u$ such that:

(a) $u$ is connected to all $v$ belong to $C_{S}$

(b) Neigbbors $(u)-C_{S}=N$ eighbors $\left(C_{S}\right)-u$

(4) $C_{S}=C_{S} \cup\{u\}$

(4) repeat (3) and (4) as long as such $u$ exists

— End of Algorithm - 
Given a graph $G$, we use the above approach to find all its critical cliques. To build a critical clique graph $C_{G}$, for any two critical cliques $C_{S}[i]$ and $C_{S}[j]$, if an edge exists between any node in $C_{S}[i]$ and any node in $C_{S}[j]$ in the original graph $G$, then an edge exists between $i$ and $j$ in the critical clique graph $C_{G}$.

\subsection{Implementation}

\subsubsection{Experimental setup}

Experiments were performed on a Dell OptiPlex GX280 using a 3.2GHz Pentium 4 dual processor, with 1.0 gigabytes (GB) of SDRAM, and running a Linux 2.6.8-2-686smp kernel. Algorithms were implemented in $\mathrm{C}$ and compiled using gcc version 3.4.4. Unless otherwise stated, we used as input synthetic graphs for which we know the optimum edit distances. For this, we built a graph generator which operates as follows. Our graph generator takes the following as input parameters: the desired number of vertices $(n)$, clusters $(c)$ and the required edit distance $(d)$. First, a random size (number of vertices) in the range $[0.5(n / c), 1.5(n / c)]$ is assigned to each cluster. We produce a clique graph $G^{\prime}$ containing $c$ fully connected cliques (clusters) of the sizes determined above, with no edges between those cliques. Then, we execute $d$ random edits on $G^{\prime}$ resulting in an output graph $G$. An edit consists of randomly inserting or deleting an edge. More precisely, for each edit we randomly decide whether to insert or delete an edge. For an insert operation, we randomly choose two vertices $i$ and $j$ that are not connected by an edge and then add an edge $(i, j)$. For a delete operation, we randomly select an edge in the graph and remove that edge. Once an edge is inserted in $G$ it cannot be deleted by a future edit. Similarly once an edge has been deleted from $G$ it cannot be re-inserted by another edit. For random number generation we used the Mitchell-Moore algorithm described in [95]. Note that, the above method creates in most cases, but not always, a graph $G$ with edit distance $d$. In some cases, as observed in our test runs, the edit distance of $G$ is smaller than $d$ because a different set of clusters than those used by our generator can be created with fewer edits. 


\subsubsection{Experimental results}

The results of our experimental evaluation of the LP and greedy methods are shown in Figures 4.5 and 4.6. Our experiments show that the LP-based cluster editing method is consistently better than the greedy method with respect to both the computation time and the value for $k$ obtained.

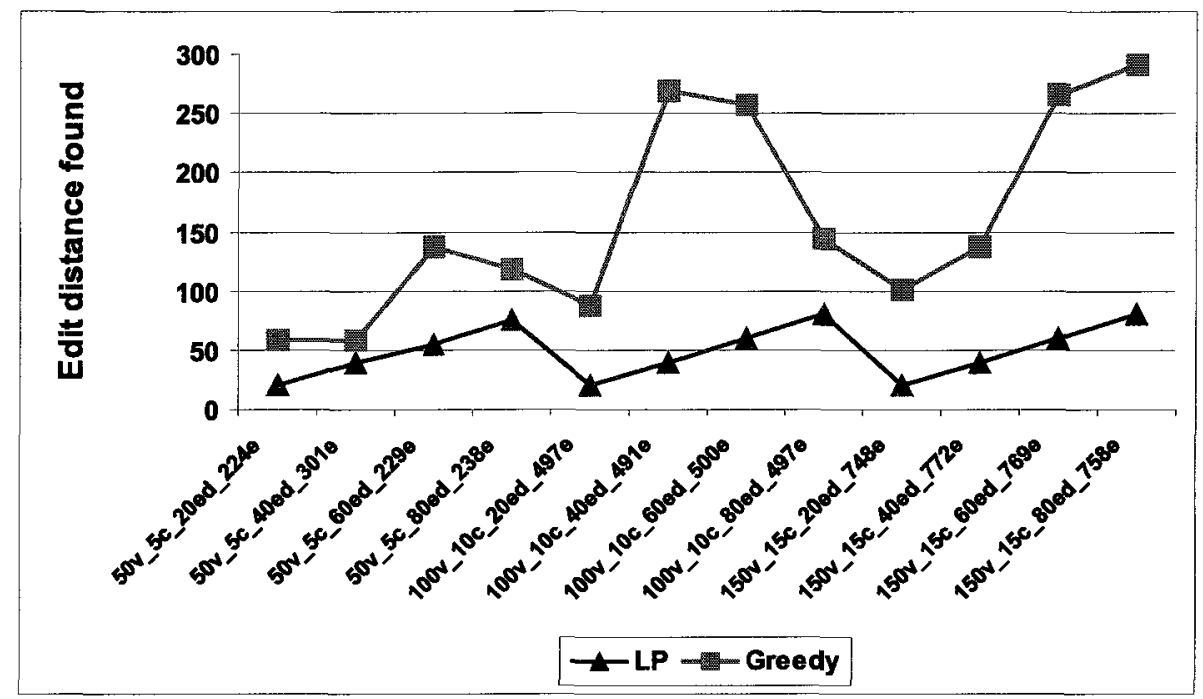

Figure 4.5: A comparison of edit distances computed by LP versus the greedy method.

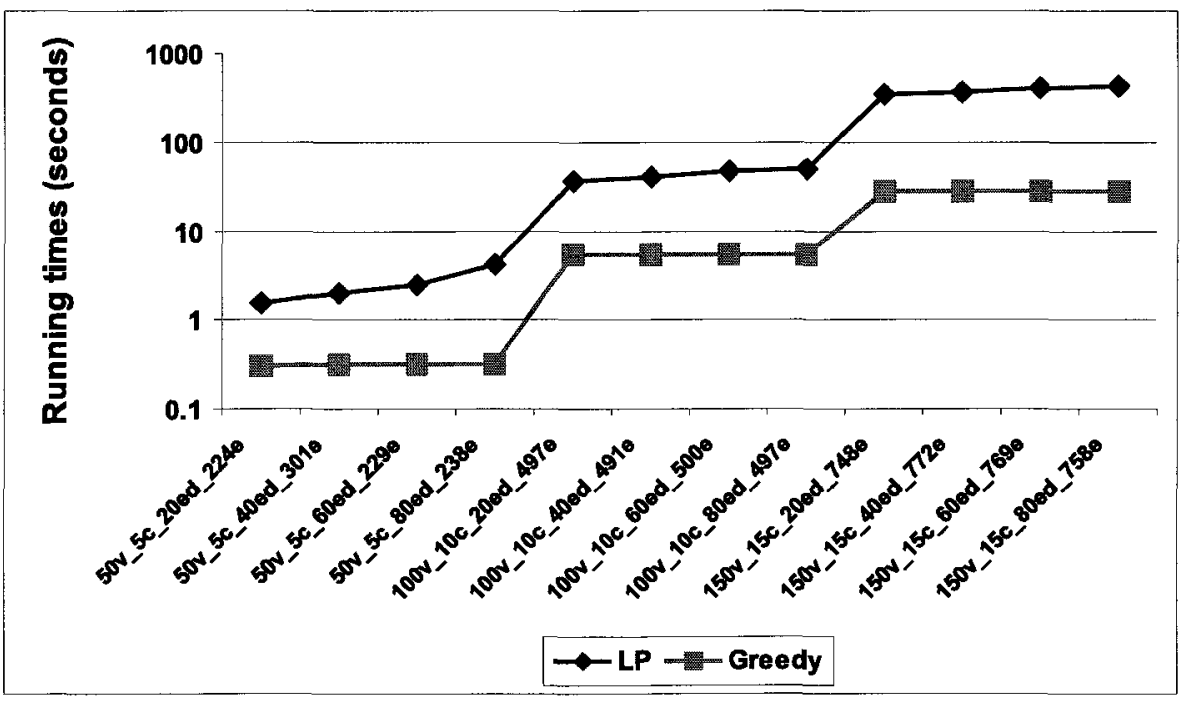

Figure 4.6: A comparison of run times required by LP versus the greedy method. 
In all cases we report branching times only because the time needed for initial preprocessing and kernelization is insignificant compared to that required during branching. However, in the experiments with interleaving, the runtime for branching includes kernelization.

It seems that refined branching is vastly superior to basic branching. The run times reported in Figure 4.7, where the edit distance is set to 20, are typical of those we observe. This was not obvious in advance. It is simply not always the case that asymptotically faster methods in the worst case translate into better algorithms in the average case. Unless the data is contrived, additional overhead and complexities incurred by ever more sophisticated branching strategies can ofttimes negate any real gains in efficiency.

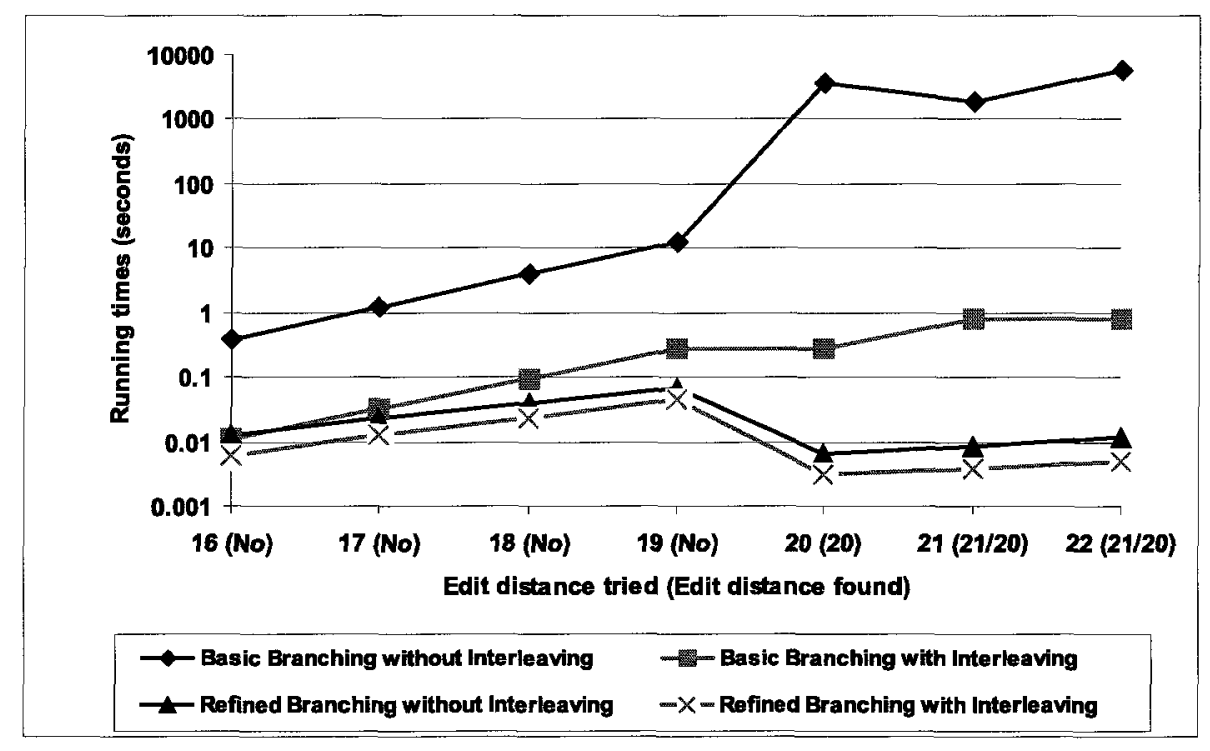

Figure 4.7: FPT run times on a graph with 50 vertices, 5 clusters and edit distance 20 .

It also seems that branching with interleaving is decidedly faster than branching without interleaving. The run times reported on larger instances in Figure 4.8, where the edit distance is set to 40, are typical. Again, this makes sense, but is neither obvious nor necessarily the case in general.

Of course we do not know the optimum edit value in advance, and so generally determine it by performing a binary search. One might expect run times to be rather 


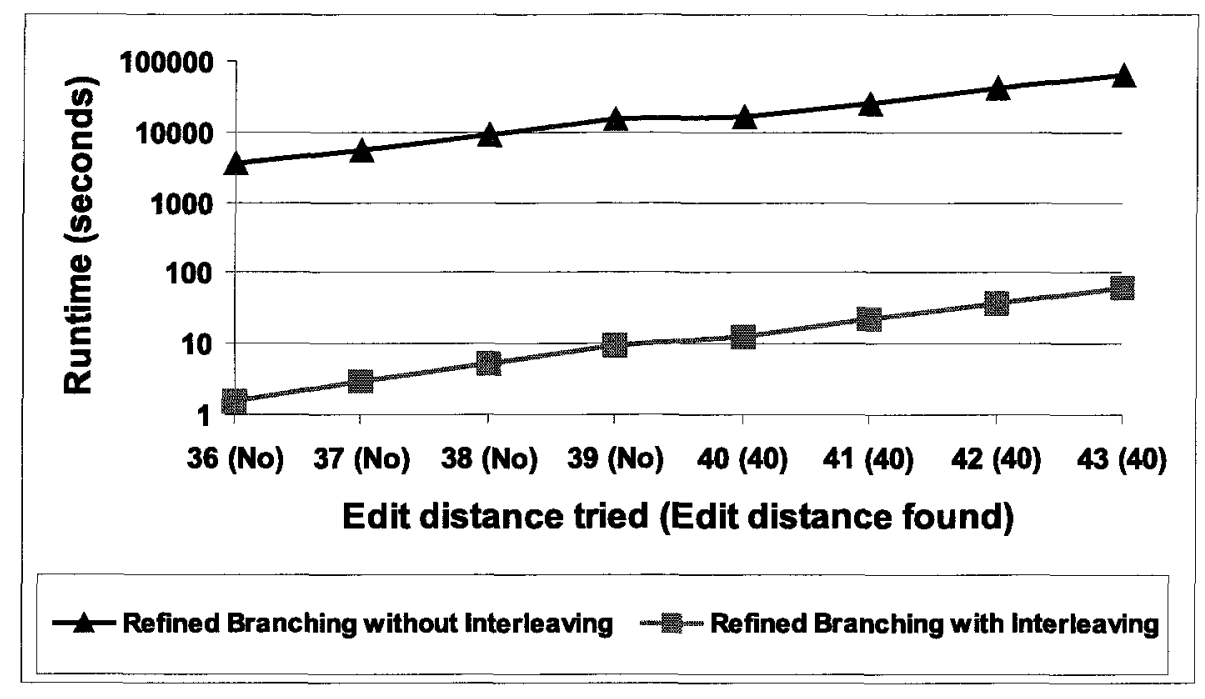

Figure 4.8: FPT run times on a graph with 100 vertices, 10 clusters and edit distance 40.

predictable. With vertex cover, for example, the most difficult computations are centered around the point at which a "no" instance becomes a "yes" instance [1, 2], with the "no" the harder of the two (with "no," our algorithms cannot find a solution and halt early). Because we are interested in clique, the situation is reversed but still monotonic above and below the optimum value. A standard example is illustrated in Figure 4.9, which was generated by Yun Zhang's another project.

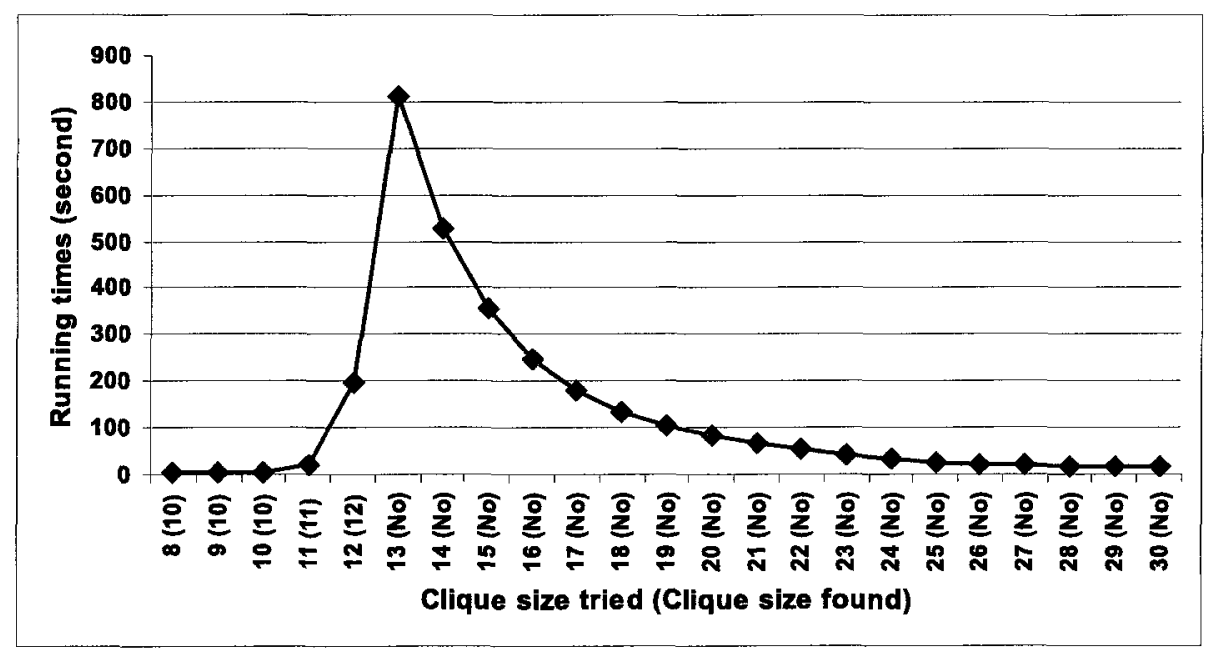

Figure 4.9: The monotonicity of FPT parameter effects as seen when solving clique with vertex cover. 
Things turn out to be quite different with cluster editing. If we happen to be advancing from below (that is, solving a "no" instance), then as with vertex cover run times predictably increase with rising parameter values. On the other hand, if we are advancing from above (facing a "yes" instance), then run times may decrease, increase or even stay the same with sinking parameter values. See Figures 4.10 and 4.11 .

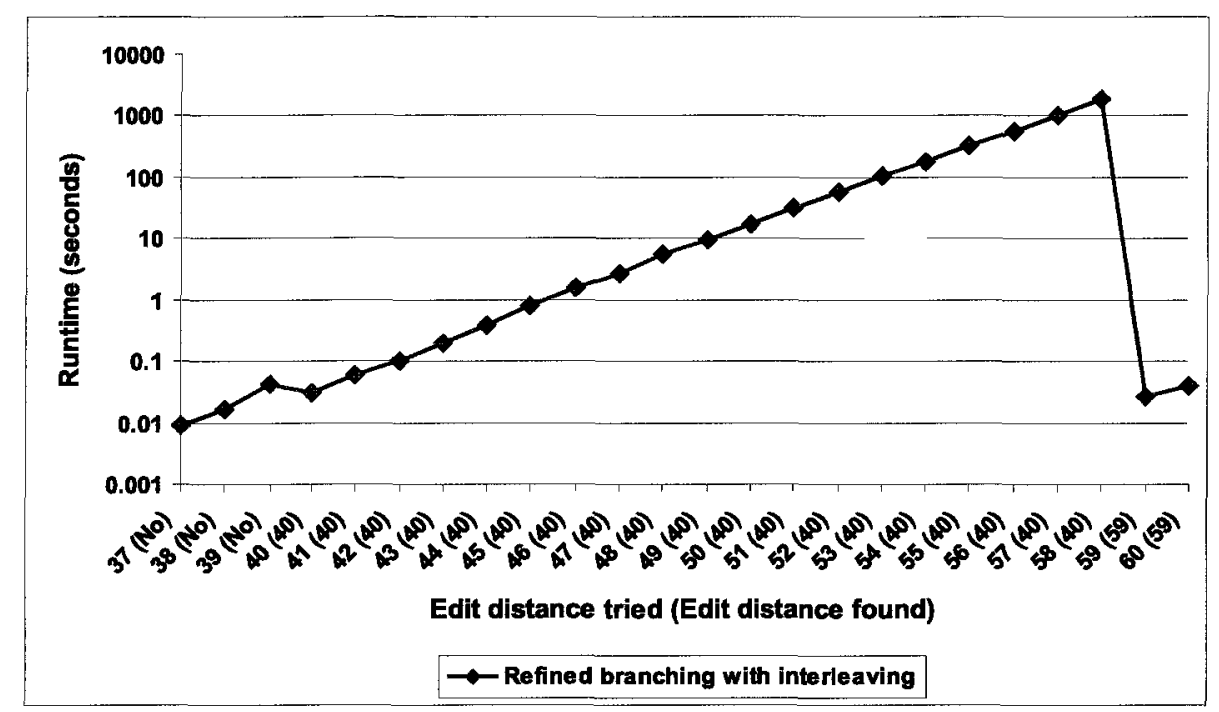

Figure 4.10: FPT parameter effects on a graph with 100 vertices, 5 clusters and edit distance 40 .

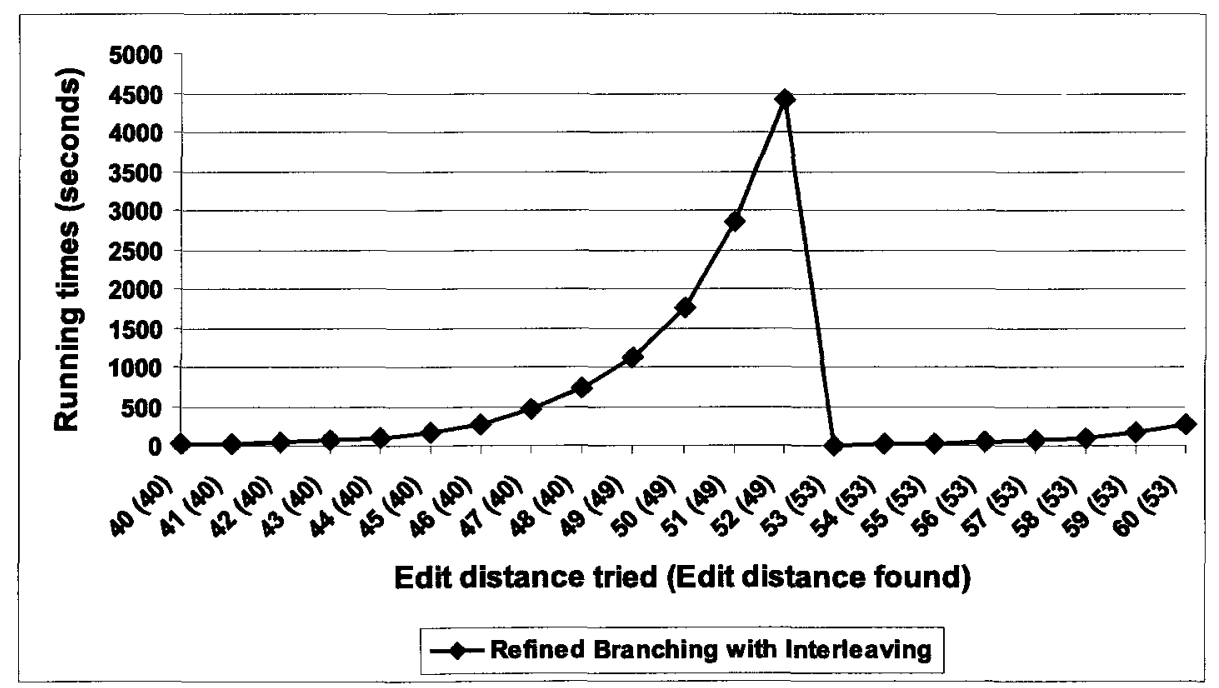

Figure 4.11: FPT parameter effects on a graph with 100 vertices, 10 clusters and edit distance 40 . 
In retrospect, we find the answer to this conundrum lies in the way solutions are distributed and the way branching works with parameter values above the optimum. With vertex cover, for example, a cover of size $i$ ensures a cover of size $i+1$ (as long as $i<|V|)$. With cluster editing, however, it is conceivable that there is a solution with edit distance $i$ yet no solution with distance $i+1$ (or $i+2$ and so forth). Thus a higher parameter value may mean only that a cluster editing algorithm has to do more work. See Figure 4.12, which depicts search tree traversals on the graph used to report run times in Figure 4.11. From this we conclude that a binary search may not be the best way to implement an FPT-based approach for clustering editing, and that one may be better off steering the parameter as much as possible from below.

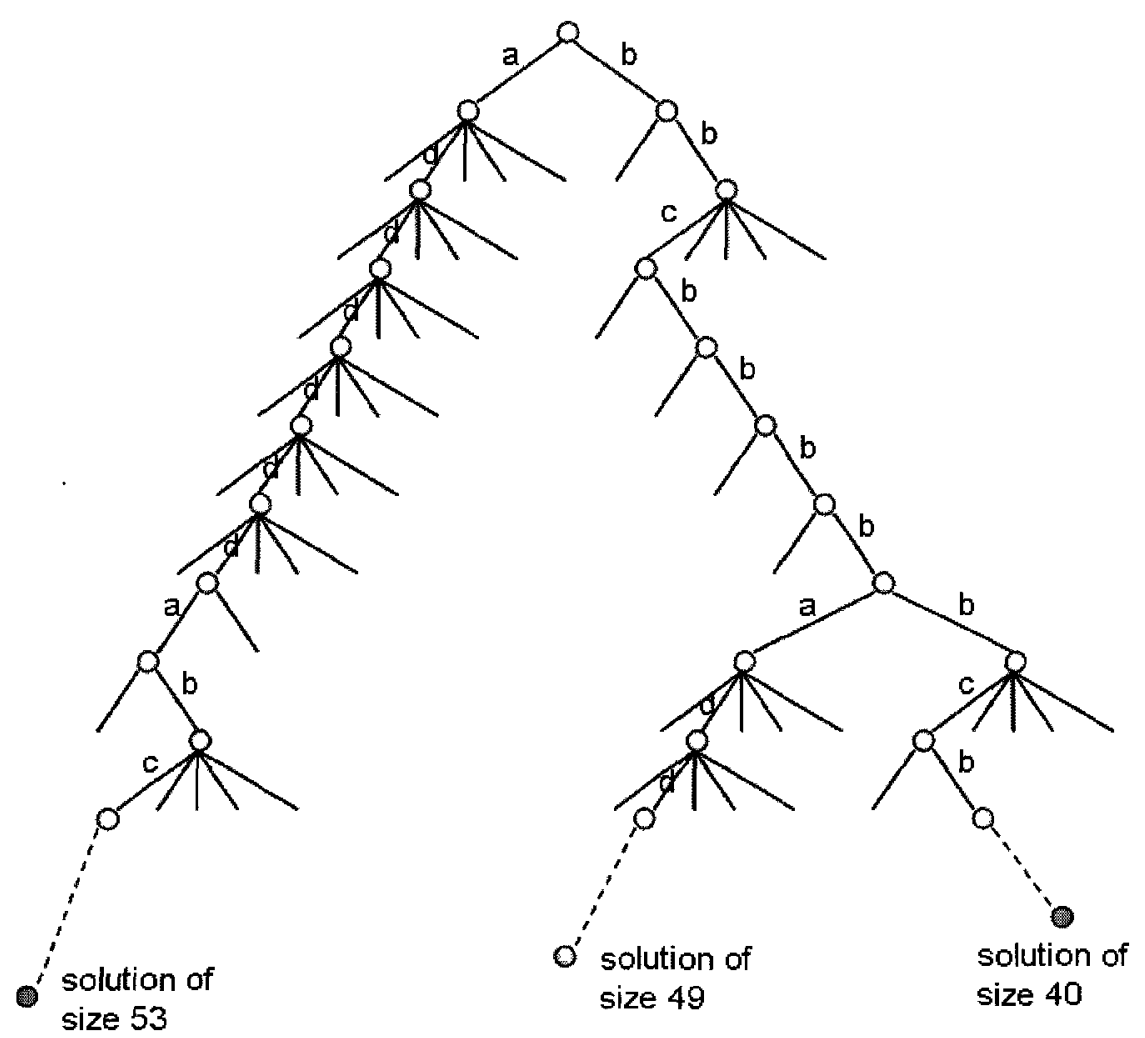

Figure 4.12: Search trees depend on parameter values.

The issue of scalability deserves scrutiny, especially if we are to scale to genomesized problem instances. Even for covers and cliques, supercomputers and monolithic memory may be heavily taxed [189]. In this respect, it is noteworthy that well-known problems such as vertex cover require searching a parameter space of size $|V|$, while 
cluster editing possesses a search space of size $|E|$. At some problem size, of course, approximation should better optimization. The exact size probably depends on many factors, including graph density, relative efficiency of implementations, and even machine architecture. Initial experiments have produced interesting comparisons. See Figure 4.13.

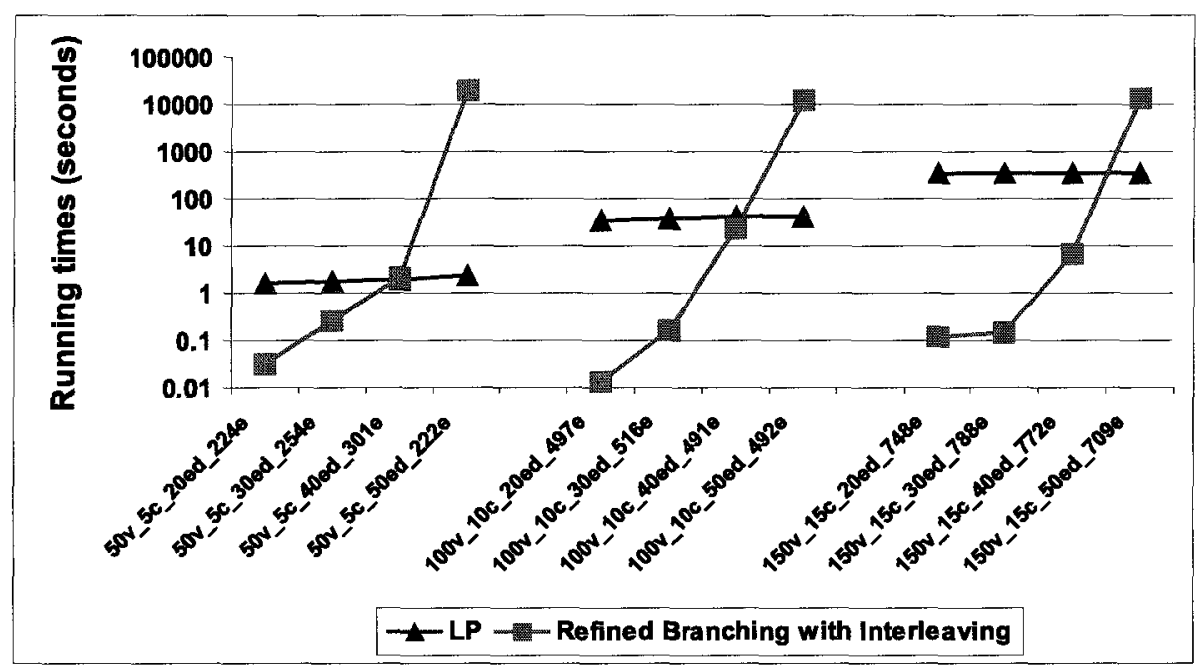

Figure 4.13: The scalability of approximation via LP versus optimization via FPT.

\subsection{Improvements}

After the above implementation was published by [37], we then developed a number of algorithms to improve the sequential performance of the FPT-based method for cluster editing. These include a greedy method for choosing branching orders(improvement method 1), a dynamically updated matrix for the number of common neighbors of a vertex pair(improvement method 2), and an LP method for choosing branching orders (improvement method 3). In addition, a parallel FPT-based method was developed to further reduce the computation time.

\subsubsection{A greedy method for choosing branching orders}

As discussed in Section 4.2.3.2, given a graph $G=(V, E)$, and a $P_{3}=\{u, v, w\}$ with edges $(u, v)$ and $(u, w)$, there are three branching rules: delete edge $(u, v)$, delete 
edge $(u, w)$, and add edge $(v, w)$. The order to perform these three branches was not considered in $[56,57]$. However, it is important to choose a branch first which is more likely to lead to a solution in order to improve the performance.

In the refined branching strategy, there are three cases:

(1) $v$ and $w$ do not share a common neighbor other than $u$;

(2) $v$ and $w$ have a common neighbor $x$ other than $u$, and $x$ is adjacent to $u$;

(3) $v$ and $w$ have a common neighbor $x$ other than $u$, but $x$ is not adjacent to $u$.

All the above cases have two branching rules: delete edge $(u, v)$ and delete edge $(u, w)$. Our previous experiments always delete edge $(u, v)$ first, then delete edge $(u, w)$. To optimize the FPT-based method, we use the neighborhood information of the given path $P_{3}$ to decide which edge should be deleted first. We suppose that two vertices with less common neighbors are more likely in different clusters than two vertices with more common neighbors, so the edge with less common neighbors should be deleted first.

We first calculate the number of common and non-common neighbors of $u$ and $v$ $\left(d_{u v}, d f_{u v}\right)$, and the number of common and non-common neighbor of $u$ and $w\left(d_{u w}, d f_{u w}\right)$. If $d_{u v} \leq d_{u w}$, delete edge $(u, v)$ first, and then delete edge $(u, w)$; otherwise, delete edge $(u, w)$ first, and then delete edge $(u, v)$. If $d_{u v}$ is equal to $d_{u w}$, then the edge with greater number of non-common neighbors is chosen to be deleted first. For example, in the case $C 2$ (see Figures 4.2), without the greedy method, the branching is performed in the following order: $2,5,6,7,8$. With the greedy method, if $d_{u v}>d_{u w}$, branching order would be $2,7,8,5,6$; if $d_{u v}=d_{u w}$ and $d f_{u v}<d f_{u w}$, branching order would be $2,7,8,5,6$. The branching order takes effect only when there exists a solution, so this method can improve "yes" cases, but will not optimize "no" cases.

\subsubsection{Dynamically updated matrix for neighbor information}

Because the number of common neighbors or non-common neighbors is frequently used in both kernelization rules and the rules for choosing branching orders, in order to improve the performance, it is necessary to have a matrix to store the neighbor information for each vertex pair, and dynamically update it when there is an edge addition or deletion. 
The matrix is symmetric. Each diagonal element stores the number of neighbors of vertex called vertex degree $d_{v}$, and each non-diagonal element stores the number of common neighbors of $u$ and $v$ called edge degree $d_{u v}$. The number of non-common neighbors of $u$ and $v$ called edge difference $d f_{u v}$ can be calculated as follows:

if $(u, v) \in E$

$$
d f_{u v}=d_{u}+d_{v}-2 d_{u v}-2
$$

if $(u, v) \notin E$

$$
d f_{u v}=d_{u}+d_{v}-2 d_{u v}
$$

The matrix is dynamically updated whenever an edge is added or deleted as follows:

If $E=E \cup(u, v)$, then $\forall w \in V$, if $(w, v) \in E, d_{u w} \leftarrow d_{u w}+1$;

$$
\text { if }(w, u) \in E, d_{v w} \leftarrow d_{v w}+1
$$

If $E=E \backslash(u, v)$, then $\forall w \in V$, if $(w, v) \in E, d_{u w} \leftarrow d_{u w}-1$;

$$
\text { if }(w, u) \in E, d_{v w} \leftarrow d_{v w}-1
$$

This method can improve performance for all $k$ values, including both "yes" and "no" cases.

\subsubsection{An LP method for choosing branching orders}

Given a graph $G=(V, E)$, and a $P_{3}=\{u, v, w\}$ with edges $(u, v)$ and $(u, w)$, there are three branching rules: delete edge $(u, v)$, delete edge $(u, w)$, and add edge $(v, w)$. As discussed in Section 4.5.1, a greedy method is used to choose branching orders. In fact, the LP method is better than the greedy method because the LP method searches global neighboring information, while the greedy method searches local neighboring information. Therefore, we developed an LP method to determine which edge should be branched first.

The LP method for choosing branching orders is implemented as follows: we first partition the graph into clusters using the LP method described in Section 4.3.2. If any two vertices are in the same cluster, they are defined as "in", otherwise, they are defined as "out". In case $C 1$ of the refined branching strategy, if the edges $(u, v)$ and $(u, w)$ are defined as the same, then any one of them can be deleted first; otherwise, the edge defined as "out" should be deleted first. 
In case $C 2$, there are 5 branching subcases (see Figures 4.2) and 8 different branching orders (see Table 4.2). The order of branching is determined on how LP defines the edges $(u, v),(v, w)$ and $(u, x)$. For example, if $(u, v)$ and $(v, w)$ are defined as "in" and $(u, x)$ is defined as "out", the branching subcase 2 should be implemented first because edge $(v, w)$ is added only in branching subcase 2. Since vertices $u$ and $v$ are in the same cluster, the branching subcases 5 and 6 should be implemented at the end because $(u, v)$ is deleted only in these two branching subcases. Vertices $u$ and $x$ are not in the same cluster, so the edge $(u, x)$ should be deleted. For branching subcases 7 and 8 , subcase 8 should be branched followed by subcase 7 because the edge $(u, x)$ is deleted in branching subcase 8 , but not in subcase 7 . Similarly, for branching subcases 5 and 6 , subcase 6 should be branched first because the edge $(u, x)$ is deleted in branching subcase 6 , but not in subcase 5 . Therefore, the branching order for this example is: $2,8,7,6,5$.

For case $C 3$ of the refined branching strategy, the branching orders are summarized in Table 4.3. Note that all subcases in Table 4.3 and Table 4.2 are labeled with the same ID as in Figures 4.2 and Figures 4.3.

\begin{tabular}{cccc}
\hline$(v, w)$ & $(u, v)$ & $(u, x)$ & Branching oder \\
\hline in & in & in & $2,7,8,5,6$ \\
in & in & out & $2,8,7,6,5$ \\
in & out & in & $2,5,6,7,8$ \\
in & out & out & $2,6,5,8,7$ \\
out & in & in & $7,8,5,6,2$ \\
out & in & out & $8,7,6,5,2$ \\
out & out & in & $5,6,7,8,2$ \\
out & out & out & $6,5,8,7,2$ \\
\hline
\end{tabular}

Table 4.2: The branching order for case $C 2$ of the refined branching strategy based on the LP method for choosing branching order. Case $C 2$ is shown in Figures 4.2.

\subsubsection{A parallel FPT-based method for cluster editing}

The basic structure of the parallel FPT-based method is a two-level master/slave model (see Algorithm 16 and Algorithm 17). A single scheduler process is in charge of dividing the branching search tree to a certain number of sub-trees and then splitting the jobs of branching to the worker processors in packets. Each packet contains a 


\begin{tabular}{cccc}
\hline$(u, v)$ & $(u, w)$ & $(u, x)$ & Branching oder \\
\hline in & in & in & $8,7,6,5,2$ \\
in & in & out & $7,8,5,6,2$ \\
in & out & in & $5,6,8,7,2$ \\
in & out & out & $5,6,7,8,2$ \\
out & in & in & $2,8,7,6,5$ \\
out & in & out & $2,7,8,5,6$ \\
out & out & in & $2,5,6,8,7$ \\
out & out & out & $2,5,6,7,8$ \\
\hline
\end{tabular}

Table 4.3: The branching order for case $C 3$ of the refined branching strategy based on the LP method for choosing branching order. Case $C 3$ is shown in Figures 4.3.

sub-tree and each worker executes refined branching on the subtree received from the scheduler process. When a worker finishes its job, it will request more work from the scheduler process and continue to work. If a worker finds a solution, it will immediately notify the scheduler processor, and then the scheduler processor will notify all other processors to stop computation. If all sub-trees are searched by processors and no solution is found by a processor at the end, the graph will be considered as "no" solution found with the current $k_{\text {tried }}$ value. It should be noted however that if the packet size is too small then the amount of communication between the scheduler and worker processes will negatively impact the running time of the system. It is therefore important to balance the packet size between being too small (too much communication overhead) and too large (too much work imbalance). However, the branching searched tree can only be divided into subtrees to a certain level because it is unknown how deep the search tree should be branched with a solution in advance. Thus, it is difficult to split the branching search tree into a balanced load.

Algorithm 16 Parallel FPT-based method for cluster editing: Scheduler

Split the branching searched tree into a certain number of packets

ndone $=0$

while ndone < nworker do

receive message from worker $\mathrm{x}$

if message $=$ NO_SOLUTION then 
ndone++

else if message $=$ YES_SOLUTION then

send KILL_SIGNAL to all workers

write YES results to output file

BREAK

while packets remain do

send packet to worker $\mathrm{x}$

send KILL_SIGNAL to all workers

write NO results to output file

— End of Algorithm -

Algorithm 17 Parallel FPT-based method for cluster editing: Worker

current_packet $=0$

current_results $=0$

work_available $=$ TRUE

foreach worker in parallel do

while work_available do

if current_packet $=0$ then

request work from scheduler

receive message from scheduler

if message $=$ NO_MORE_WORK or KILL_SIGNAL then

work_available $=$ FALSE

BREAK

current_packet $=$ message

retrieve subtree from current.packet

run refined branching on subtree

if result $=$ YES_SOLUTION

write result to output file

send message to scheduler

BREAK

send NO_SOLUTION to scheduler 
current_packet $=0$

- End of Algorithm -

\subsubsection{Experimental results after further improvements}

First, graph $100 v \_10 c \_40 e d \_491 e$ used in [37] was tested to compare the new implementation with the previous one. As shown in Figure 4.14 and Figure 4.15, the runtime of the new implementation was decreased significantly, and also smaller edit distance was found sooner. Figure 4.15 shows that in the old implementation without improvement methods, three different edit distances $\left(k_{\text {found }}\right)$ were found: 40,49 and 53 when $k_{\text {tried }}$ increases from 40 to 60 . For the new implementation with the greedy method for choosing branching orders (improvement methods 1) and dynamically updated matrix for neighbor information (improvement method 2), however, $k_{\text {found }}$ is always 40 , which is the optimum edit value. Figure 4.12 shows the search tree of the graph. Without the new improvement methods, the branch order is performed as follows: solution size of 53,49 , and 40 , so if the value of parameter $k_{\text {tried }}$ is larger than or equal to 53 , the edit distance $k_{\text {found }}$ would be 53 . If $k_{\text {trred }}$ is smaller than 53 but larger than 49 , then the edit distance would be 49 , and so on. However, with the new optimization methods, the branch of solution size of 40 is performed first, so when parameter $k_{\text {tried }}$ is larger than 40 , the edit distance $k_{\text {found }}$ would be 40 , which is the optimum edit distance. Therefore, with the new improvement methods 1 and 2 , the optimum edit distance can be found quicker for graph 100v_10c_40ed_491e.

We then implemented these two improvement methods on a small but dense graph. This graph (20v_95e) contains 20 vertices and 95 edges with unknown edit distance. Figure 4.16 depicts the runtimes for the "no" cases of $k_{\text {tried }}$ from 20 to 59 (right portion of the figure) and the "yes" cases of $k_{\text {trzed }}$ from 60 to 95 (left portion of the figure). It is apparent that there is a steady rise in the runtime when $k_{\text {treed }}$ increases for the "no" cases or decreases for the "yes" cases, although there are some step-shapes on the "yes" side. Note that the optimal edit value is unknown in advance. Figure 4.16 shows that the closer $k_{\text {tried }}$ is to the optimal edit distance(60), the longer it takes to get an output. This is different from what we observed in the previous experiments. This new implementation shows that a binary search might be a practical way to 


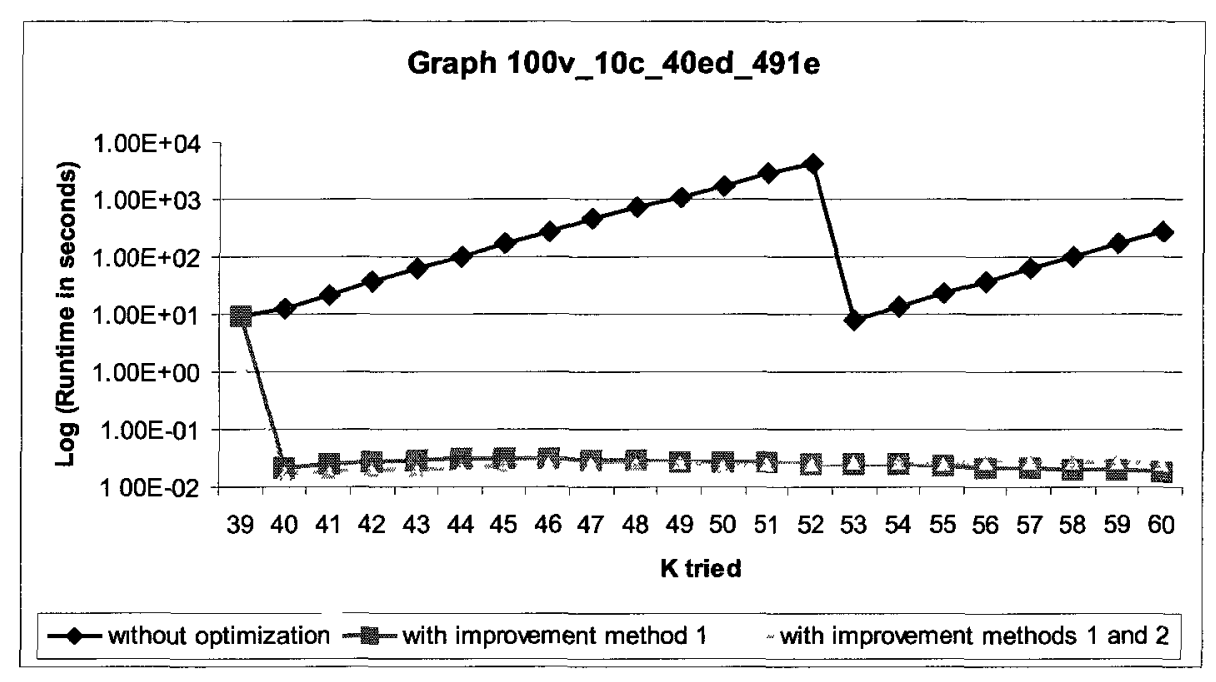

Figure 4.14: A comparison of run time before and after the FPT improvement methods.

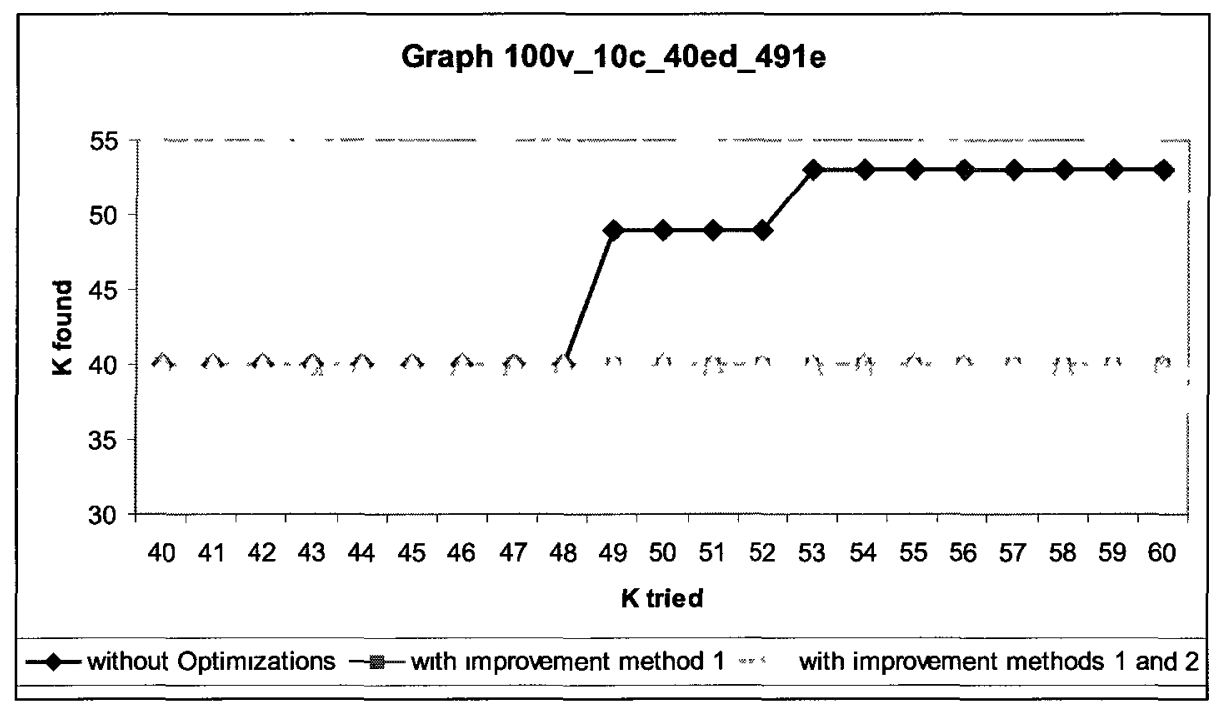

Figure 4.15: A comparison of edit distances computed for Yes case before and after the FPT improvement methods.

implement the improved FPT-based approach for clustering editing.

Figures 4.17 and 4.18 show the comparison of runtime between the LP method and the greedy method in choosing branching orders. In these two implementations, dynamically updated matrix was also used to store neighbor information. As shown in these two figures, compared with the greedy method in choosing branching orders, the LP method decreased the runtime greatly, especially for graph $20 v \_95 e$. These 


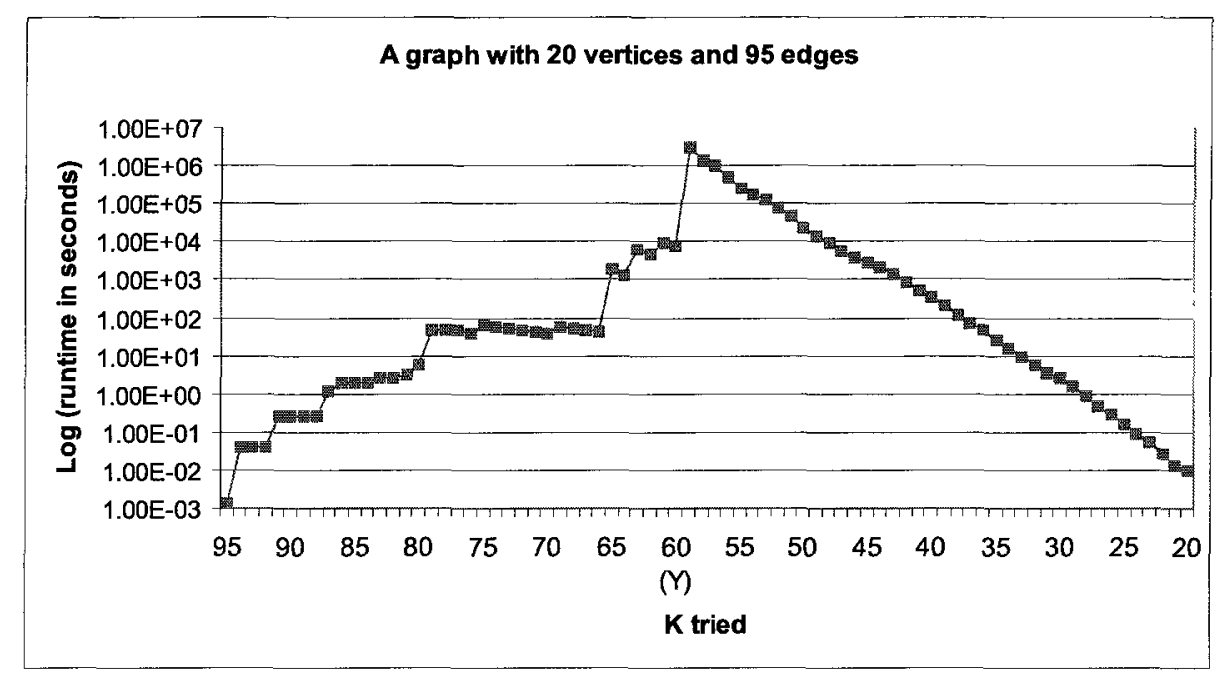

Figure 4.16: FPT run times on a graph with 20 vertices and 95 edges.

results further confirmed that the LP method is better than the greedy method in choosing branching orders.

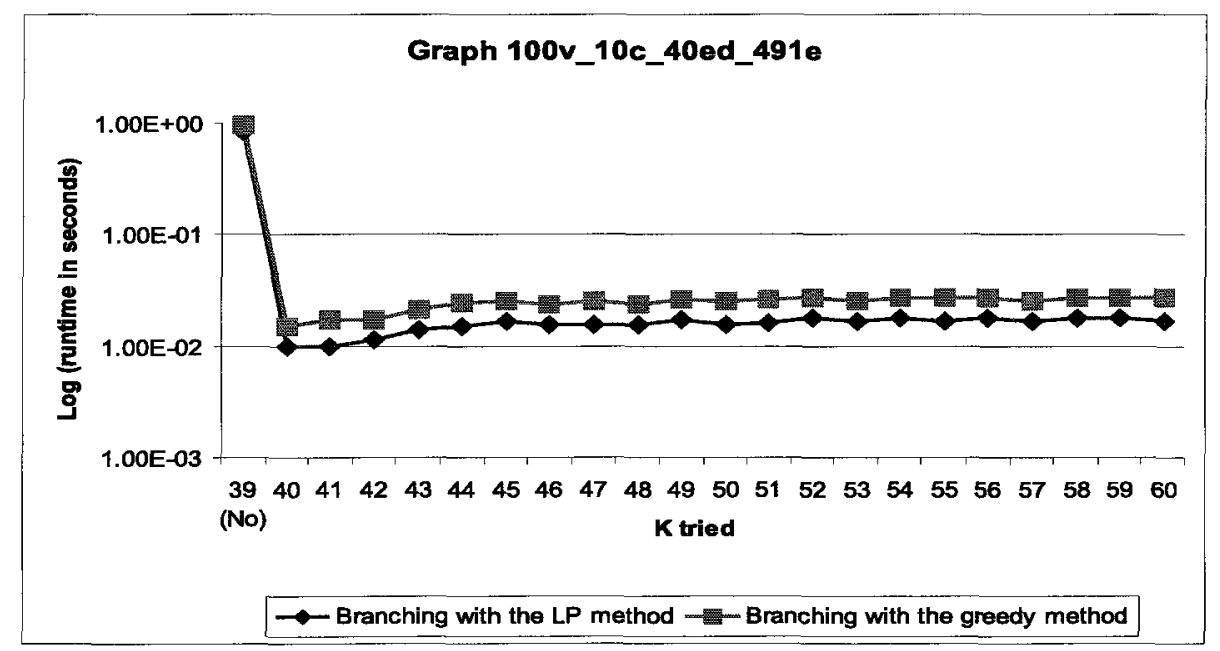

Figure 4.17: A comparison of run time on graph 100v_10c_40ed_491e between the LP method and the greedy method for choosing branching orders.

To further reduce the computation time, the FPT-based method was executed on a 200-processor cluster instead of a single workstation. As shown in Figure 4.18, in general the parallel method decreased the runtime, especially for "no" cases. The closer $k_{\text {tried }}$ is to the optimal edit distance, the more runtime is reduced. However, for some "yes" cases, the parallel method did not reduce the runtime. This is because the 


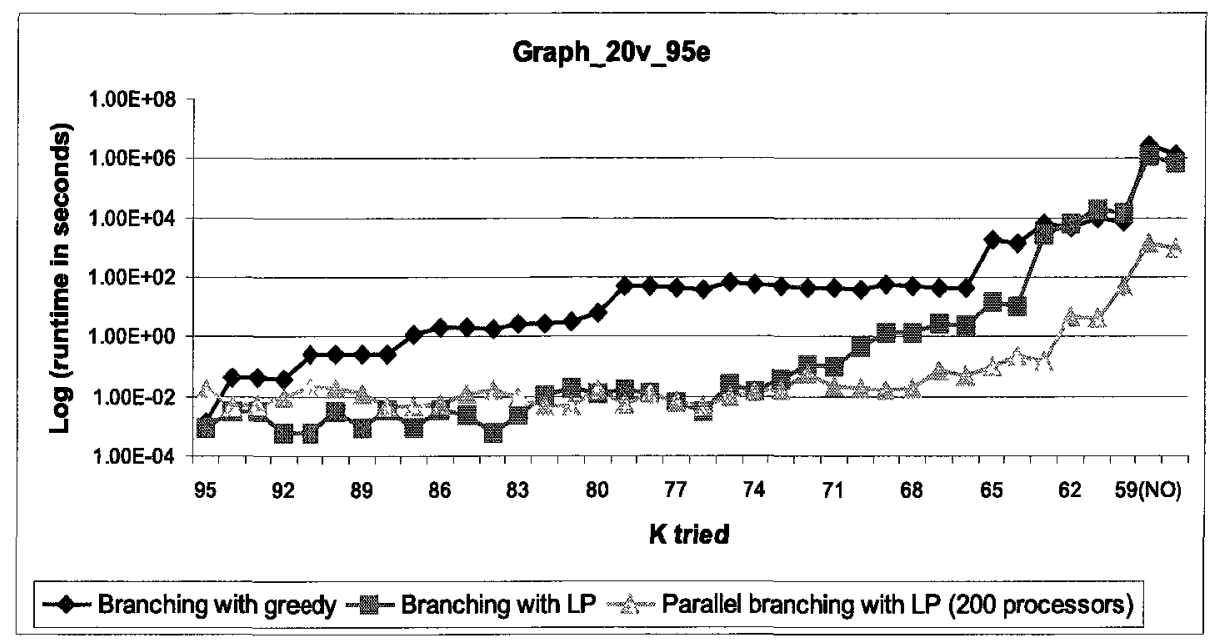

Figure 4.18: A comparison of run time on graph 20v_95e among the LP method, the greedy method and the parallel method for branching.

branching jobs are not equally assigned to the processors. Ideally, the jobs in parallel methods should be equally divided so that each processor is assigned a same load of work. However, it is difficult to split the jobs into a balanced load. Some processors will get more computation to perform than the others, and those processors with less work load will sit idle until the loaded processors complete. The runtime for cluster editing with a "yes" case depends on how quickly a branching tree with a solution can be searched by a processor. Due to unbalanced work load, it is likely that a solution will not be found sooner on parallel machines than on a single machine. However, for "no" cases, all sub-trees need to be searched so the performance on parallel machines should be better than that of a single machine.

Although we substantially improved the FPT-based method for cluster editing, this method can only solve graphs of small sizes. Even for a small but very dense graph, the program can not solve the problem within a reasonable time. For example, for a graph with 81 vertices and 269 edges (Graph 21 in Table 4.8), the edit distance was found to be 170 when $k_{\text {tried }}$ was set to 170 (see Figure 4.19). However, when $k_{\text {tried }}$ was set to 160 , the program did not generate any results after running on a 200 processor cluster for 8 days. Until now the optimal edit distance for this graph is still unknown.

To further improve the performance of this FPT-based method for cluster editing, 


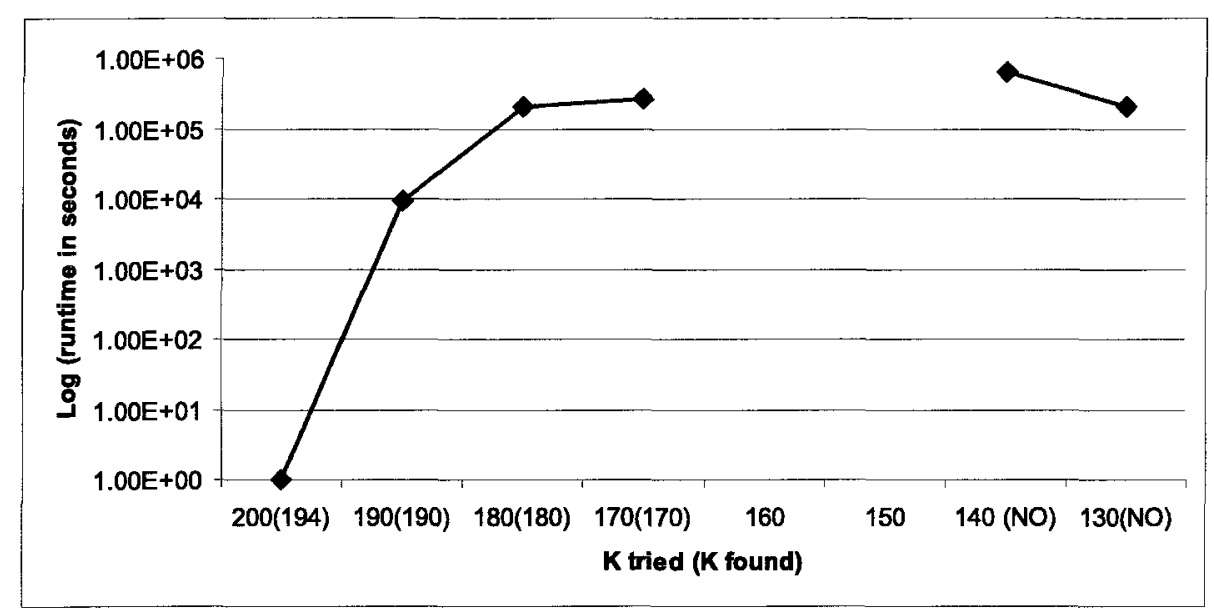

Figure 4.19: The run time on the graph $81 v \_269 e$ by the parallel FPT-based method.

we implemented Guo's new linear kernelization rules described in Section 4.2.3.3 to reduce the problem kernel. We first converted the graphs to critical clique graphs using the method describe in Section 4.3.3.2. The new kernelization rule (Rule 2 in Section 4.2.3.3) is applied to the critical graphs, but it is to our surprise that none of the tested graphs has a vertex that satisfies Rule 2. Therefore, the new kernelization rule is not able to reduce the problem kernels of the tested graphs. Thus, it cannot improve the performance of our cluster editing program.

\subsection{Application}

Protein-protein interactions (PPI) are fundamental to biological processes within a cell. With the rapid development in system biology and cell biology, large amounts of data focused on physical and genetic protein interactions have been generated. Physical interactions are defined as direct interactions between molecules, and genetic interactions are defined as functional relationships through cause-and-effect relationships between genes [8]. Both of these types of interactions can indicate shared or associated/related protein functions. The network of interactions between proteins is generally modeled as an interaction graph, where nodes represent proteins and edges correspond to interactions. Beyond individual interactions, a lot of systematic information are contained in protein interaction graphs as represented by protein complexes and regulatory pathways $[137,144]$. 
A protein complex is a group of two or more proteins that highly physically interact with each other. It corresponds to a dense subgraph in the PPI networks [7, 141]. Protein complexes carry out many important molecular functions. Therefore, identification of protein complexes can help to understand and explain certain biological processes. A number of experimental techniques have been applied to discover protein complexes including yeast two-hybrid and affinity purification/mass spectrometric identification methods such as TAP-tagging. These methods are all labour and time consuming and have a high cost associated with them, which has resulted in a growing need for the development of computational tools that are capable of predicting protein complexes. With the increase of the sizes of PPI networks, efficient and accurate methods to predict protein complexes on PPI graphs have become of great importance. Such prediction can serve as a guide to biological experiments to discover novel protein complexes.

A number of approaches have been reported to predict protein complexes from PPI networks. Most of these methods are based on clique finding [165, 35, 30] and clustering [90, 39, 46, 7, 15]. As most of them are heuristic methods, these methods cannot generate an optimal solution. It is therefore necessary to develop a methodology to predict protein complexes with an optimal solution.

\subsubsection{The cluster editing method to predict protein complexes in $S$. cere- visiae}

We applied our cluster editing method to the PPI networks of the yeast Saccharomyces cerevisiae to identify and predict protein complexes. We first built a protein graph generator for cluster editing. The interaction database used for generating protein graphs is the physical yeast interactions from the General Repository of Interaction Datasets (BioGRID). BioGRID is a database that contains genetic and physical protein-protein interactions among proteins from 13 species [167]. Interactions are regularly added through exhaustive curation of the primary literature.

The protein graph generator operates as follows. It takes two input parameters: a protein $(P)$ and the number of vertices $(v)$. Based on the protein $P$, the first step is to find $v$ proteins for the protein graph by searching the yeast interaction database. 
Starting from $P$, the program searches the interaction database and finds its neighboring proteins. The neighboring proteins are those proteins that have interactions with the searched protein. For every neighboring protein, the program performs the same search as for $P$. When the total number of proteins reaches $v$, the program stops searching. In the second step, the program generates a protein graph based on all searched proteins: vertices represent the searched proteins, and edges represent interactions. For any two vertices $i$ and $j$, if there is an interaction between them in the interaction database, an edge $(i, j)$ will be added into the graph.

The cluster edit distance for graph $G$ is the smallest value of $k$ for which cluster editing is possible. It is also the smallest value of $k_{\text {found }}\left(k_{\text {min }}\right)$. In order to find the cluster edit distance of $G$, we used the LP-based cluster editing method described in Subsection 4.3.2 to determine $k_{L P}$. As discussed in Subsection 4.3.3, the lower bound and the upper bound of the optimal edit distance are $k_{L P} / 4$ and $k_{L P}$, respectively. Based on the search interval $\left[k_{L P} / 4, k_{L P}\right]$, we run the FPT-based cluster editing method on $G$ through binary searching on $k$. The final graph, which is the output of cluster editing with $k_{\min }$ edge edition, consists of a number of disjoint cliques. Although a protein complex often involves more than two proteins, two proteins can also form a complex. However, there is a difference between two proteins that interact and form a complex, and two proteins that interact without leading to a complex. A protein complex is defined by a well characterized function, while protein interaction does not have to have a defined function. In this study, the minimum size of the predicted protein complexes is chosen as 4 in order to increase the chances of getting a complex and not just interactions. Therefore, the cliques whose size is smaller than 4 will be discarded in the final graph and the rest cliques are considered as our predicted complexes. In the remainder of this thesis, predicted and known protein complexes are referred to as clusters and complexes, respectively.

The result of this cluster editing method is a graph that consists of a disjoint union of cliques, so this method does not allow overlaps of protein complexes. However, it is possible that there are partial overlaps of protein complexes in PPI networks [188]. Although most proteins (around 73\%) are members of a single complex, some of them are shared by different complexes [18]. Therefore, when the cluster editing method 
is used to predict protein complexes, some will be left out due to the requirement of disjoint union of cliques in the final graph. In order to allow overlapping of cliques, we developed a new method called Cluster Editing with Overlapping as follows.

Given a graph $G$, we first use the cluster editing method to find protein cliques $C_{S}$, and then delete the edges of $C_{S}$ (not including the edited edges) from the original graph $G$. For the new Graph $G^{\prime}$, we run the cluster editing method again to find more cliques. Since the edges of some cliques have been deleted, new cliques might be found when the second round cluster editing is performed.

\subsubsection{Experimental results}

\subsubsection{Test graphs}

We first generated 19 protein graphs of yeast. For each graph, the input protein $P$ was randomly chosen from the database and the size of the graph is set to 45 proteins. We executed the cluster editing method on these test graphs to identify and predict protein complexes. Among 19 test protein graphs, 16 graphs were solved by cluster editing and 48 clusters were found. The other 3 protein graphs were not solved by cluster editing within a week and therefore the program was terminated.

We compared the protein clusters found by the cluster editing method with known protein complexes [125]. The database was obtained from Munich Information center for Protein Sequences (MIPS) [124] (ftp://ftpmips.gsf.de/yeast/catalogues/complexcat). 43 among the 48 protein clusters identified in our method have more than $50 \%$ overlaps with the known complexes, and 16 clusters share some common biological features. Table 4.4 shows three examples that correlate best with the known complexes. The biological features were obtained from a software called YEAST FEATURES (http://software.dumontierlab.com/yeastfeatures/).

Protein cluster $A$ in Table 4.4 matches a known protein complex. The MIPS $20 \mathrm{~S}$ proteasome complex has 15 proteins that are all present in cluster $A$. Compared with the 20S proteasome complex, a new protein YHR200W was found in cluster $A$. All the proteins in cluster $A$ except YBR173C have peptidase activity, and 12 proteins including the novel protein are involved in the protein modification processes. This suggests that the new protein YHR200W may have similar functions with other 


\begin{tabular}{|c|c|c|c|}
\hline $\begin{array}{l}\text { Protein clusters found } \\
\text { by cluster editing }\left(C_{S}\right)\end{array}$ & $\begin{array}{l}\text { Known proteln } \\
\text { complexes }\left(C_{S}^{\prime}\right)\end{array}$ & $\begin{array}{l}\text { No of } \\
\text { common } \\
\text { proteins }\end{array}$ & Biological features of $C_{S}$ \\
\hline $\begin{array}{l}\text { Cluster } A \text { (s1ze 16) } \\
\text { YBL041W YBR173C } \\
\text { YER012W YER094C } \\
\text { YFR050C YGL011C } \\
\text { YGR135W YGR253C } \\
\text { YJL001W YML092C } \\
\text { YMR314W YOL038W } \\
\text { YOR157C YOR362C } \\
\text { YPR103W YHR200W }\end{array}$ & $\begin{array}{c}\text { 20S proteasome (size 15) } \\
\text { YBL041W YBR173C } \\
\text { YER012W YER094C } \\
\text { YFR050C YGL011C } \\
\text { YGR135W YGR253C } \\
\text { YJL001W YML092C } \\
\text { YMR314W YOL038W } \\
\text { YOR157C YOR362C } \\
\text { YPR103W }\end{array}$ & 15 & $\begin{array}{c}\text { Function } \\
\text { Peptidase activity } \\
\text { (All except YBR173C) } \\
\text { Process } \\
\text { Protein modification } \\
\text { (12 Proteins including YHR200W) }\end{array}$ \\
\hline $\begin{array}{l}\text { Cluster } B \text { (size 7) } \\
\text { YML105C(SEC65) } \\
\text { YPR088C(SRP54) } \\
\text { YPL243W(SRP68) } \\
\text { YPL210C(SRP72) } \\
\text { YKL122C(SRP21) } \\
\text { YDL092W(SRP14) } \\
\text { YDL051W(LHP1) }\end{array}$ & $\begin{array}{l}\text { SRP (size 6) } \\
\text { YDL092W YKL122C } \\
\text { YML105C YPL210C } \\
\text { YPL243W YPR088C }\end{array}$ & 6 & $\begin{array}{c}\text { Process } \\
\text { Transport(SRP14, SRP 21, } \\
\text { SEC65, SRP72, SRP 68 and SRP54) } \\
\text { Complex } \\
\text { ribonucleoprotein complex } \\
\text { (SEC65, SRP14, SRP21, SRP54, } \\
\text { SRP68 and SRP72) } \\
\text { Compartement } \\
\text { Cytoplasm (SRP14, SEC65, } \\
\text { SRP72, SRP68, SRP52) }\end{array}$ \\
\hline $\begin{array}{l}\text { Cluster } C \text { (size 6) } \\
\text { YDL225W(SHS1) } \\
\text { YLR314C(CDC3) } \\
\text { YJR076C(CDC11) } \\
\text { YHR107C(CDC12) } \\
\text { YDR507C(GIN4) } \\
\text { YCR002C(CDC10) }\end{array}$ & $\begin{array}{l}\text { Septin filaments (size } 7 \text { ) } \\
\text { YCR002C YDL225W } \\
\text { YDR218C YGR059W } \\
\text { YHR107C YJR076C } \\
\text { YLR314C }\end{array}$ & 5 & $\begin{array}{c}\text { Function } \\
\text { Structural molecule activity } \\
\text { (CDC3, CDC10, CDC11, CDC12, SHS1) } \\
\text { Process } \\
\text { Cytokinesis (CDC3, CDC10, CDC11, CDC12, SHS1) } \\
\text { Cell wall organization and biogenes1s } \\
\text { (CDC3, CDC10, CDC11, CDC12) } \\
\text { Function for the new protein YDR507C(GIN4) } \\
\text { Protein kinase involved in bud growth } \\
\text { assembly of the septin ring } \\
\text { kinase-dependent and kinase-1ndependent activities } \\
\text { undergoes autophosphorylation }\end{array}$ \\
\hline
\end{tabular}

Table 4.4: Comparison of protein clusters found by cluster editıng with known protein complexes. 
proteins in cluster $A$ and may be also involved in the same process as other proteins in cluster $A$. Since functions and processes are important criteria in deciding biological interactions, it is very likely that YHR200W has interactions with other proteins in cluster $A$.

Protein cluster $B$ in Table 4.4 that contains 7 proteins matches the MIPS signal recognition particle (SRP) complex that has 6 proteins. All 6 proteins in the SRP complex are contained in cluster $B$, and all of them are involved in the transport process. The remaining protein in cluster $B$ is a novel protein YDL051W. Based on the clustering relationship, YDL051W can be predicted to be involved in the transport process as well.

Protein cluster $C$ matches the MIPS septin filaments complex. There are 5 common proteins between cluster $C$ and MIPS septin filaments complex. Also, cluster $C$ found a novel protein YDR507C (See Table 4.4). All the 5 common proteins have a function as structural molecules, and they are involved in the cytokinesis processes. The novel protein YDR507C has a number of biological functions: protein kinase involved in yeast growth; assembly of the septin ring; kinase-dependent and kinaseindependent activities; and undergoes autophosphorylation. Although the biological relevance of these clusters remains to be confirmed by biological experiments, our computational results indicated that our cluster editing method has potential not only to identify known protein complexes, but also to predict new proteins in existing complexes.

We also examined the new protein interactions predicted by our cluster editing method. For protein cluster $A$ in Table 4.4, there are 11 edges which were not in the original protein graph, but were added by the cluster editing method. These edges are defined as predicted edges. The database used for cluster editing is the BioGRID database published on July 26th 2006. Compared with the BioGRID database as of December 2007, 7 predicted edges have been reported as interactive and one more predicted edge has been added in the BioGRID database as of October 1st, 2008 (see Table 4.5). Among the 11 protein edges predicted by our cluster editing program, 8 $(73 \%)$ of them were later confirmed by biochemical experiments. This confirmed that our cluster editing method has potential to predict new edges in PPI networks. 


\begin{tabular}{ll}
\hline Predicted protein interactions & Confirmed \\
\hline YOR362C - YER094C & BioGRID (Dec. 2007) \\
YMR314W - YJL001W & BioGRID (Dec. 2007) \\
YMR314W - YER094C & BioGRID (Dec. 2007) \\
YJL001W - YER094C & BioGRID (Dec. 2007) \\
YJL001W - YDL188C & \\
YJL001W - YBR173C & BioGRID (Dec. 2007) \\
YGR253C - YER094C & BioGRID (Dec. 2007) \\
YFR050C - YBR173C & \\
YER094C - YBL041W & BioGRID (Dec. 2007) \\
YDL188C - YBR173C & \\
YBR173C - YBL041W & BioGRID (Oct. 1st, 2008) \\
\hline
\end{tabular}

Table 4.5: Comparison of predicted edges with newly updated protein-protein interaction database.

To examine cluster editing with overlapping method in the prediction of protein complexes, similar to previous experiment, we randomly chose a protein from the database as a starting vertex and generated 6 test graphs with 45 proteins for each. Table 4.6 shows a summary of implementation of cluster editing with overlapping on 6 test graphs. The second round cluster editing found one more clusters in four graphs, but not in the other two (Test Graphs 4 and 5). For Test Graph 2, three protein clusters (Cluster 1, 2 and 3 in Table 4.7) were found by the first round cluster editing. After deleting the edges of these three clusters in the original graph, one more protein cluster (Cluster 4 in Table 4.7) was found by the second round cluster editing. It occurs that Cluster 4 partially overlaps with Clusters 3 (YNL298W and YDR309C), and with Cluster 1 (YLR229C). This method allows the overlaps between protein clusters and thus has potential to find more protein clusters.

The above test graphs were generated from a random protein. In addition, to further predict new proteins in known complexes of interest, we implemented cluster editing on some protein graphs generated from a list of 26 proteins that are involved in non-homologous end-joining process. Based on these 26 proteins, we first found 21 MIPS complexes that contain at least two of these proteins. Then based on each of those 21 know complexes, we expanded the protein graphs to contain all its neighboring proteins from the interaction database. The size of the protein graphs varies from 27 to 538 , and the number of edges varies from 63 to over 4600 (see Table 4.8). 


\begin{tabular}{lcc}
\hline & $\begin{array}{c}\text { Number of clusters found } \\
\text { after the first round cluster editing }\end{array}$ & $\begin{array}{c}\text { Number of clusters found } \\
\text { after the second round cluster editing }\end{array}$ \\
\hline Test Graph 1 & 1 & 1 \\
Test Graph 2 & 3 & 1 \\
Test Graph 3 & 1 & 1 \\
Test Graph 4 & 2 & 0 \\
Test Graph 5 & 1 & 0 \\
Test Graph 6 & 2 & 1 \\
\hline
\end{tabular}

Table 4.6: Summary of the implementation of cluster editing method with overlapping on 6 test graphs.

\begin{tabular}{ll}
\hline & Protein cluster \\
\hline 1 & YLR229C YJL157C YHL007C YER114C YBR200W YAL041W \\
2 & YPL242C YNL271C YFL039C YCL027W \\
3 & YNL298W YHR061C YDR309C YHR107C \\
4 & YLR229C YOR127W YNL298W YDR309C \\
\hline
\end{tabular}

Table 4.7: Protein clusters predicted for Test Graph 2 after the first and the second round cluster editing.

Among those 21 protein graphs, we implemented cluster editing on 6 graphs whose sizes are relatively small (Graphs 4, 7, 15, 17, 18 and 21). The edit distances found for these 6 graphs were summarized in Table 4.9. Although some edit distances were found by the FPT-based cluster editing method, no optimal edit distance $\left(k_{m i n}\right)$ was found after running the program for about a month (see Table 4.9).

The test graphs generated from one protein are mainly centered around the starting protein (vertex). Such graphs are relatively sparse or require a small number of edits, therefore the cluster editing method can solve them and find corresponding optimal edit distances within a reasonable period of time. For the graphs generated from protein complexes, they are denser than those from one protein, therefore solving these graphs may require more edge edits and much more running time to transfer into a cluster graph.

\subsection{Conclusions and future work}

We implemented an FPT-based method for cluster editing using the approach described in $[56,57]$. This is the first implementation of an FPT based method for 


\begin{tabular}{lccc}
\hline Graph No. & Size of known complexes & Size of the graph & Number of edges \\
\hline Graph 1 & 31 & 538 & 4646 \\
Graph 2 & 28 & 454 & 3032 \\
Graph 3 & 20 & 351 & 2152 \\
Graph 4 & 4 & 84 & 295 \\
Graph 5 & 19 & 473 & 3199 \\
Graph 6 & 24 & 422 & 2711 \\
Graph 7 & 10 & 124 & 709 \\
Graph 8 & 14 & 184 & 977 \\
Graph 9 & 8 & 197 & 722 \\
Graph 10 & 9 & 255 & 901 \\
Graph 11 & 5 & 243 & 942 \\
Graph 12 & 6 & 259 & 1058 \\
Graph 13 & 8 & 237 & 1111 \\
Graph 14 & 3 & 63 & 114 \\
Graph 15 & 6 & 104 & 272 \\
Graph 16 & 2 & 27 & 63 \\
Graph 17 & 4 & 61 & 137 \\
Graph 18 & 4 & 71 & 219 \\
Graph 19 & 2 & 81 & 130 \\
Graph 20 & 3 & 70 & 226 \\
Graph 21 & 4 & 81 & 269 \\
\hline
\end{tabular}

Table 4.8: Summary of the graphs generated from a list of proteins that are involved in non-homologous end-joining process.

\begin{tabular}{|c|c|c|}
\hline Graph No. & $k_{L P}$ & $k_{\text {tried }}\left(k_{\text {found }}\right)$ \\
\hline 4 & 295 & $295(290) 250(250) 240(?) 90($ no $) 80($ no $)$ \\
7 & 875 & $875(659) 600(?)$ \\
15 & 462 & $180(170) 160(?)$ \\
17 & 98 & $78(78) 75(?) 70($ no $)$ \\
18 & 124 & $124(121) 110(?) 50($ no $)$ \\
21 & 565 & $350(220) 200(194) 170(170) 160(?) 140($ no $)$ \\
\hline
\end{tabular}

Table 4.9: Summary of the edit distances found by cluster editing for the protein graphs that are involved in non-homologous end-joining process. 
cluster editing. In order to evaluate the effectiveness of the FPT approach, we also implemented an LP-based method and a greedy method for cluster editing. Our experiments showed that the best results for cluster editing were obtained by using the refined branching method with interleaving, which was vastly superior to the basic branching method. We also demonstrated that, in practice, branching with interleaving was indeed faster than branching without interleaving. For cluster editing, we did not know the optimum edit distance in advance, so we used binary search of $k$. Our experimental results later confirmed that a binary search is a practical way to implement the FPT based approach for cluster editing.

We also developed a number of methods to improve the performance of the FPTbased method for cluster editing, a greedy method for choosing branching orders, a dynamically updated matrix for neighbor information and an LP method for choosing branching orders. With the new optimization methods, the performance of the FPTbased method was greatly improved. In addition, we also parallized the method on a cluster.

Finally, we applied our cluster editing method to the PPI networks of yeast to identify and predict protein complexes. The comparison of the protein clusters found by our method with known yeast protein complexes showed that our method has potential to identify known protein complexes and also to predict new proteins in the complexes. The cluster editing method predicted new edges in a PPI graph. By comparing these edges with the newly updated database, nearly $73 \%$ of them were later confirmed by biochemical experiments. These results confirmed that our cluster editing program has potential to predict new edges in PPI networks as well.

Despite of this substantially improved FPT-based method for cluster editing, our current method is limited to solve graphs of small sizes. Even for a small but very dense graph, our program may not be able to solve the problem within a reasonable period of time. This indeed represents a limitation of the cluster editing method in predicting protein complexes and protein interactions. Future work will improve the performance of the method in order to implement cluster editing on a larger graph. 


\section{Chapter 5}

\section{Summary of contributions}

This thesis presented computational methods for three different biological problems: pool design for in vitro selection of complex aptamers; identification of TIPs by computational comparative analysis of next generation personal genome data; and identification of protein complexes from protein-protein interaction networks.

We developed two computational methods to generate RNA/DNA sequences that exhibit higher structural complexity and that can be used to increase the overall structural diversity of initial pools for in vitro selection experiments [111]. Random Filtering selectively increases the number of five-way junctions in RNA/DNA pools, and Genetic Filtering designs RNA/DNA pools to a specified structure distribution, whether uniform or otherwise. Biological experiment showed that using our computationally designed DNA pool greatly improved access to highly complex sequence structures for SELEX experiments without losing the ability to select simple structures with low degrees of complexity. Random Filtering and Genetic Filtering have proved to be useful tools for biologists in designing the starting pool in order to enhance the possibility of finding novel aptamers with improved activity.

We developed a new algorithm called TIP-finder to identify TIPs from personal genomes [110]. We tested the efficiency of TIP-finder using a simulated genome and are able to detect about $88 \%$ of TIPs with a precision of $\geq 91 \%$. The precision of TIPs_OUT prediction does not seem to be affected by the low genome coverage, while the sensitivity tends to drop slightly along with the decreasing genome coverage. The fact that a genome sequence data at a coverage as low as $5 \mathrm{X}$ still allows detection of more than $84 \%$ of true TIPs_OUT suggests that low coverage personal genome data that are currently available at a much larger number than those at deep coverage due to the lower cost can also be valuable for the prediction of structural variations. Using TIP-finder to analyze six genomes representing two trio families, we identified a 
total of 5569 TIPs, consisting of 4881, 456, 91, and 141 insertions from Alu, L1, SVA and HERV, respectively. Some TIPs predicted by this method have been confirmed by PCR experiment. In addition to the estimated insertion location, TIP-finder also provides prediction of TE subfamily, insertion size and genotype for each TIP, which can be very valuable for the downstream experimental validation and analysis. This study represented one of the few comprehensive analysis of TIPs performed so far and revealed an unexpectedly highly level of transposon associated genetic polymorphsim in humans.

We presented the first practical implementation of a FPT-based method for cluster editing which is a NP-hard problem [37]. We also improved this algorithm by using techniques to optimize its performance. We then applied this cluster editing method to identify and predict protein complexes of yeast. This is the first attempt to use the cluster editing method to predict protein complexes. The comparison of the protein clusters found by our method with known yeast protein complexes showed that this method has potential to identify known protein complexes and to predict new proteins in the complexes. Since the FPT-based cluster editing method provides the optimum solution for cluster editing, our current method can only solve graphs of small sizes. Even for a small but very dense graph, the method may not solve the problem within a reasonable time. Further research is needed to validate the method and to improve its performance so that we can implement cluster editing on a larger graph.

In summary, we have demonstrated that high performance computational approaches can be implemented to provide highly meaningful perspectives in complex biological systems. These tools have become indispensable in bioinformatics research. 


\section{Bibliography}

[1] F. N. Abu-Khzam, R. L. Collins, M. R. Fellows, M. A. Langston, W. H. Suters, and C. T. Symons. Kernelization algorithms for the vertex cover problem: Theory and experiments. In Proceedings, Workshop on Algorithm Engineering and Experiments, New Orleans, Louisiana, 2004.

[2] F. N. Abu-Khzam, M. A. Langston, P. Shanbhag, and C. T. Symons. Scalable parallel algorithms for fpt problems. Algorithmica, 45:263 - 267, 2006.

[3] N. Ailon, M. Charikar, and A. Newman. Aggregating inconsistent information:ranking and clustering. In Proc. 37th ACM STOC, pages 684-693. Springer LNCS 4169, 2006.

[4] S. F. Altschul, W. Gish, W. Miller, E. W. Myers, and D. J. Lipmanl. Basic local alignment search tool. Journal of Molecular Biology, 215:403-410, 1990.

[5] S. Arcot, T. Shaikh, J. Kim, L. Bennett, M. Alegria-Hartman, D. Nelson, P. Deininger, and M. Batzer. Sequence diversity and chromosomal distribution of "young" alu repeats. Gene, 163:273 - 278, 1995.

[6] D. V. Babushok, E. M. Ostertag, and H. H. K. Jr. Current topics in genome evolution: molecular mechanisms of new gene formation. Cell Mol Life Sci, $64: 542-554,2007$.

[7] G. Bader and C. Hogue. An automated method for finding molecular complexes in large protein interaction networks. BMC Bioinformatics, 4(2), 2003.

[8] S. Bandyopadhyay, R. Kelley, N. J. Krogan, and T. Ideker. Functional maps of protein complexes from quantitative genetic interaction data. PLoS Computational Biology, 4:e1000065, 2008.

[9] N. Bansal, A. Blum, and S. Chawla. Correlation clustering. In Proc. of 43rd FOCS, pages $238-247,2002$.

[10] R. T. Batey, S. D. Gilbert, and R. K. Montange. Structure of a natural guanineresponsive riboswitch complexed with the metabolite hypoxanthine. Nature, $432: 411-415,2004$.

[11] M. Batzer, S. Arcot, J. Phinney, M. Alegria-Hartman, D. Kass, S. Milligan, C. Kimpton, P. Gill, M. Hochmeister, P. Ioannou, R. Herrera, D. Boudreau, W. Scheer, B. Keats, P. Deininger, and M. Stoneking. Sequence diversity and chromosomal distribution of "young" alu repeats. Journal of molecular evolution, 42:22 - 29, 1996. 
[12] M. Batzer and P. Deininger. Alu repeats and human genomic diversity. Nature reviews, 3:370 - 379, 2002.

[13] A. D. Baxevanis and B. F. F. Ouellette. Bioinformatics: A Practical Guide to the Analysis of Genes and Proteins. Wiley-Interscience, 2005.

[14] E. Bindewald, R. Hayes, Y. G. Yingling, W. Kasprzak, and B. A. Shapiro. Rnajunction: a database of rna junctions and kissing loops for three-dimensional structural analysis and nanodesign. Nucleic acids research, 36:D392 - D397, 2008.

[15] M. Blatt, S. Wiseman, and E. Domany. Superparamagnetic clustering of data. Physical Review Letters, 76(18):3251-3254, 1996.

[16] H. L. Bodlaender, M.R.Fellows, P. Heggernes, F.Mancini, C. Papadopoulos, and F. Rosamond. Clustering with partial information. In Proc. MFCS, pages 144 - 155. Springer LNCS 5162, 2008.

[17] R. R. Breaker. Natural and engineered nucleic acids as tools to explore biology. Nature, 432(7019):838-845, 2004.

[18] S. Brohee and J. Helden. Evaluation of clustering algorithms for protein-protein interaction networks. BMC Bioinformatics, 7:488-507, 2006.

[19] T. Brown. Genomes. John Wiley and Sons Inc., 2002.

[20] L. Cai. Fixed-parameter tractability of graph modification problems for hereditary properties. Information Processing Letters, 58:171-176, 1996.

[21] P. A. Callinan, J. Wang, S. W. Herke, R. K. Garber, P. Liang, and M. A. Batzer. Alu retrotransposition-mediated deletion. J Mol Biol, 348:791 - 800, 2005.

[22] P. Campbell and et al. Identification of somatically acquired rearrangements in cancer using genome-wide massively parallel paired-end sequencing. Nature genetics, 40:722-729, 2008.

[23] J. M. Carothers, J. H. Davis, and et al. Solution structure of an informationally complex high-affinity rna aptamer to gtp. $R N A, 12(4): 567-579,2006$.

[24] J. M. Carothers, S. C. Oestreich, and et al. Informational complexity and functional activity of rna structures. J Am Chem Soc, 126(16):5130-5137, 2004.

[25] M. Charikar, V. Guruswami, and A. Wirth. Clustering with qualitative information. Journal of Computer and System Sciences, 71:360-383, 2005. 
[26] J. M. Chen, N. Chuzhanova, P. D. Stenson, and C. F. andD. N. Cooper. Metaanalysis of gross insertions causing human geohuchi04ostertagostertag01netic disease, novel mutational mechanisms and the role of replication slippage. Hum. Mutat., 25(2):207 - 221, 2005.

[27] K. Chen and et al. Breakdancer: An algorithm for high resolution mapping of genomic structural variation. Nature methods, 6:677-681, 2009.

[28] D. Chiang and et al. High-resolution mapping of copy-number alterations with massively parallel sequencing. Nature methods, 6:99-103, 2009.

[29] W. Chiuman and Y. Li. Evolution of high-branching deoxyribozymes from a catalytic dna with a three-way junction. Chemistry and Biology, 13:1061-1069, 2006.

[30] H. N. Chua, K. Ning, and et al. Using indirect protein-protein interactions for protein complex prediction. J Bioinform Comput Biol., 6(3):435-466, 2008.

[31] D. Conrad, T. Andrews, N. Carter, M. Hurles, and J. Pritchard. A highresolution survey of deletion polymorphism in the human genome. Nature genetics, 38:75-81, 2006.

[32] G. Cooper, D. Nickerson, and E. Eichler. Mutational and selective effects on copy-number variants in the human genome. Nature genetics, 39:S22-S29, 2007.

[33] G. Cooper, T. Zerr, J. Kidd, E. Eichler, and D. Nickerson. Systematic assessment of copy number variant detection via genome-wide snp genotyping. Nature genetics, 40:1199-1203, 2008.

[34] R. Cordaux and M. Batzer. The impact of retrotransposons on human genome evolution. Nat Rev Genet, 10:691 - 703, 2009.

[35] G. Cui, Y. Chen, D. Huang, and K. Han. An algorithm for finding functional modules and protein complexes in protein-protein interaction networks. Journal of Biomedicine and Biotechnology, 2008:860270, 2008.

[36] J. H. Davis and J. W. Szostak. Isolation of high-affinity gtp aptamers from partially structured rna libraries. PNAS, 99(18):11616-11621, 2002.

[37] F. Dehne, M. A. Langston, X. Luo, S. Pitre, P. Shaw, and Y. Zhang. The cluster editing problem: Implementations and experiments. In Proceedings, International Workshop on Parameterized and Exact Computation, pages 13 24. Springer LNCS 4169, 2006.

[38] E. D. Demaine and N. Immorlica. Correlation clustering with partial information. In Proc. of 6th APPROX, pages 1-13. Springer LNCS 2764, 2003. 
[39] S. V. Dongen. Graph clustering by flow simulation. PhD thesis, University of Utrecht, 2000.

[40] R. G. Downey and M. R. Fellows. Parameterized Complexity. Springer, 1999.

[41] J. Du, R. Bjornson, Z. Zhang, Y. Kong, M. Snyder, and M. Gerstein. Integrating sequencing technologies in personal genomics: optimal low cost reconstruction of structural variants. PLoS computational biology, 5:e1000432, 2009.

[42] R. Durbin, G. Abecasis, D. Altshuler, A. Auton, L. Brooks, R. Durbin, R. Gibbs, M. Hurles, and G. McVean. A map of human genome variation from population-scale sequencing. Nature, 467:1061 - 1073, 2010.

[43] E. Eichler, D. Nickerson, B. A. Altshuler, D., L. Brooks, N. Carter, D. Church, A. Felsenfeld, M. Guyer, C. Lee, J. Lupski, J. Mullikin, J. Pritchard, J. Sebat, S. Sherry, D. Smith, D. Valle, and R. Waterston. Completing the map of human genetic variation. Nature, 447:161 - 165, 2007.

[44] A. D. Ellington and J. W. Szostak. In vitro selection of rna molecules that bind specific ligands. Nature, 346:818 822, 1990.

[45] D. Emanuel and A. Fiat. Correlation clustering - minimizing disagreements on arbitrary weighted graphs. In Proc. of 11th ESA, pages $208-220$. Springer LNCS 2832, 2003.

[46] A. J. Enright, S. V. Dongen, and C. A. Ouzounis. An efficient algorithm for large-scale detection of protein families. Nucleic Acids Res, 30(7):1575-1584, 2002.

[47] A. Ewing, H. Kazazian, and Jr. High-throughput sequencing reveals extensive variation in human-specific 11 content in individual human genomes. Genome research, 20:1262 - 1270, 2010.

[48] M. Famulok and S. Verma. In vivo-applied functional rnas as tools in proteomics and genomics research. Trends Biotechnol, 20(11):462-466, 2002.

[49] M. Fellows, M. Langston, F. Rosamond1, and P. Shaw. Efficient parameterized preprocessing for cluster editing. In Fundamentals of Computation Theory, pages 312 - 321. Springer LNCS 4639, 2007.

[50] L. Feuk, A. Carson, and S. Scherer. Structural variation in the human genome. Nature reviews, 7:85 - 97, 2006.

[51] R. Fleischmann, M. Adams, O. White, R. Clayton, E. Kirkness, A. Kerlavage, C. Bult, J. Tomb, B. Dougherty, J. Merrick, and et al. Whole-genome random sequencing and assembly of haemophilus influenzae rd. Science, 269:496-512, 1995. 
[52] H. H. Gan, S. Pasquali, and T. Schlick. Exploring the repertoire of rna secondary motifs using graph theory; implications for rna design. Nucleic Acids Research, 31(11):2926-2943, 2003.

[53] J. Gevertz, H. Gan, and T. Schlick. In vitro rna random pools are not structurally diverse: A computational analysis. $R N A, 11: 853-863,2005$.

[54] A. Gionis, H. Mannila, and P. Tsaparas. Clustering aggregation. In Proc. 21st ICDE, pages $341-352,2005$.

[55] J. Gramm, J. Guo, F. Hueffner, and R. Niedermeier. Automated generation of search tree algorithms for hard graph modification problems. Algorithmica, $39: 321-347,2004$.

[56] J. Gramm, J. Guo, F. Hueffner, and R. Niedermeier. Graph-modeled data clustering: Fixed-parameter algorithms for clique generation. Theory of Computing Systems, 38(4):373 - 392, 2005.

[57] J. Guo. Algorithm design techniques for parameterized graph modification problems. PhD thesis, Univ. Jena, 2005.

[58] J. Guo. A more effective linear kernelization for cluster editing. In Proc. 1st ESCAPE, pages $36-47$. Springer LNCS 4614, 2007.

[59] J. S. Han, S. T. Szak, and J. D. Boeke. Transcriptional disruption by the 11 retrotransposon and implications for mammalian transcriptomes. Nature, 429:268 - 274, 2004.

[60] K. Han, S. K. Sen, J. Wang, P. A. Callinan, J. Lee, R. Cordaux, P. Liang, and M. A. Batzer. Genomic rearrangements by line-1 insertion-mediated deletion in the human and chimpanzee lineages. Nucleic Acids Res, 33:4040 - 4052, 2005.

[61] T. Hermann and D. J. Patel. Adaptive recognition by nucleic acid aptamers. Science, 287(5454):820 825, 2000.

[62] D. Hinds, A. Kloek, M. Jen, X. Chen, and K. Frazer. Common deletions and snps are in linkage disequilibrium in the human genome. Nature genetics, 38:82 $85,2006$.

[63] D. R. Hodgson and H. Suga. Mechanistic studies on acyl-transferase ribozymes and beyond. Biopolymers, 73(1):130-150, 2004.

[64] I. Hofacker. Vienna rna sccondary structure server. Nucleic Acids Res, 31(13):3429-3431, 2003.

[65] I. L. Hofacker, W. Fontana, P. F. Stadler, L. S. Bonhoeffer, M. Tacker1, and P. Schuster. Fast folding and comparison of rna secondary structures. Chemical Monthly, 125(2):167-188, 1994. 
[66] E. Hollox, U. Huffmeier, P. Zeeuwen, R. Palla, J. Lascorz, D. Rodijk-Olthuis, P. van de Kerkhof, H. Traupe, G. de Jongh, M. den Heijer, A. Reis, J. Armour, and J. Schalkwijk. Psoriasis is associated with increased beta-defensin genomic copy number. Nature genetics, 40:23 - 25, 2008.

[67] F. Hormozdiari, C. Alkan, E. Eichler, and S. Sahinalp. Combinatorial algorithms for structural variation detection in high-throughput sequenced genomes. Genome research, 19:1270-1278, 2009.

[68] F. Hormozdiari, C. Alkan, M. Ventura, I. Hajirasouliha, M. Malig, F. Hach, D. Yorukoglu, P. Dao, M. Bakhshi, S. Sahinalp, and E. Eichler. Alu repeat discovery and characterization within human genomes. Genome research, 2011 March 10.

[69] F. Hormozdiari, I. Hajirasouliha, P. Dao, F. Hach, D. Yorukoglu, C. Alkan, E. Eichler, and S. Sahinalp. Next-generation variationhunter: combinatorial algorithms for transposon insertion discovery. Bioinformatics, 26:i350-357, 2010.

[70] W. Hsu and T. Ma. Substitution decomposition on chordal graphs and applications. In Proc. 2nd International Symposium on Algorithms, pages $52-60$. Springer LNCS 557, 1991.

[71] C. Huang, A. Schneider, Y. Lu, T. Niranjan, P. Shen, M. Robinson, J. Steranka, D. Valle, C. Civin, T. Wang, S. Wheelan, H. Ji, J. Boeke, and K. Burns. Mobile interspersed repeats are major structural variants in the human genome. Cell, $141: 1171-1182,2010$.

[72] D. E. Huizenga and J. W. Szostak. A dna aptamer that binds adenosine and atp. Biochemistry, 34:656 - 665, 1995.

[73] International HapMap Consortium. A haplotype map of the human genome. 437.

[74] International HapMap Consortium. The international hapmap project. Nature, 426, 2003.

[75] International Human Genome Sequencing Consortium. Initial sequencing and analysis of the human genome. Nature, 409:860 - 921, 2001.

[76] International Human Genome Sequencing Consortium. Finishing the euchromatic sequence of the human genome. Nature, 431:931-945, 2004.

[77] F. J. Isaacs, D. J. Dwyer, and et al. Rna synthetic biology. Nat Biotechnol, 24(5):545-554, 2006.

[78] L. Jaeger, M. C. Wright, and et al. A complex ligase ribozyme evolved in vitro from a group i ribozyme domain. Proc Natl Acad Sci U S A, 96(26):14712$14717,1999$. 
[79] H. H. K. Jr. Mobile elements: drivers of genome evolution. Scrence, 303:1626 $-1632,2004$.

[80] J. Jurka, V. Kapitonov, A. Pavlicek, P. Klonowski, O. Kohany, and J. Walichiewicz. Repbase update, a database of eukaryotic repetitive elements. Cytogentıc and Genome Research, 110:462 - 467, 2005.

[81] J. Kaiser. Dna sequencing. a plan to capture human diversity in 1000 genomes. Scrence, 319:395, 2010.

[82] F. Kammer and H. Taeubig. Graph Connectuvty. Technische Univeritat Munchen, 2004.

[83] V. B. Kandimalla and H. Ju. New horizons with a multi dimensional tools for applications in analytical chemistry - aptamer. Analytıcal letters, 37(11):2215$2233,2004$.

[84] D. Khoo, C. Perez, and et al. Characterization of rna determinants recognized by the arginine- and proline-rich region of us11, a herpes simplex virus type 1-encoded double-stranded rna binding protein that prevents pkr activation. $J$ Virol, 76(23):11971-11981, 2002.

[85] J. S. Kieft, K. Zhou, A. Grech, R. Jubin, and J. A. Doudna. Crystal structure of an rna tertiary domain essential to hev ires-mediated translation initiation. Nat. Struct. Bıol., 9:370 - 374, 2002.

[86] N. Kim, H. H. Gan, and T. Schlik. A computational proposal for designing structured rna pools for in vitro selection of rnas. Bıoınformatıcs, 13:478-492, 2007.

[87] N. Kim, J. A. Izzo, S. Elmetwaly, H. H. Gan, and T. Schlick. Computational generation and screening of rna motifs in large nucleotide sequence pools. $\mathrm{Nu}$ cleıc Acıds Res, 38(13):e139, 2010.

[88] N. Kim, J. Shin, S. Elmetwaly, H. Gan, and T. Schlick. Ragpools: Rna-asgraph-pools a web server for assisting the design of structured rna pools for in vitro selection. Bionformatıcs, 23(21):2959-2960, 2007.

[89] P. Kim and et al. Analysis of copy number variants and segmental duplications in the human genome: Evidence for a change in the process of formation in recent evolutionary history. Genome research, 18:1865-1874, 2008.

[90] A. D. King, N. Przulj, and Z. Yakhini. Protein complex prediction via cost-based clustering. Bıoınformatıcs, 20:3013-3020, 2004.

[91] S. Klussmann. The Aptamer Handbook: Functıonal Olıgonucleotıdes and Therr Applıcatıons. Wiley-VCH, 2006. 
[92] S. Klussmann. The Aptamer Handbook: Functional Oligonucleotides and Their Applications, pages 94-115. Wiley, 2006.

[93] S. Klussmann. The Aptamer Handbook: Functional Oligonucleotides and Their Applications, pages 131-166. Wiley, 2006.

[94] R. Knight, H. D. Sterck, and et al. Abundance of correctly folded rna motifs in sequence space, calculated on computational grids. Nucleic Acids Res, 33(18):5924-5935, 2005.

[95] D. E. Knuth. The Art of Computer Programming, volume 2: Seminumerical algorithms. Addison-Wesley, 3 edition, 1997.

[96] M. Konkel, J. Wang, P. Liang, and M. Batzer. Identification and characterization of novel polymorphic line- 1 insertions through comparison of two human genome sequence assemblies. Gene, 390:28-38, 2007.

[97] J. Korbel and et al. Paired-end mapping reveals extensive structural variation in the human genome. Science, 318:420-426, 2007.

[98] M. Krivanek and J. Moravek. Np-hard problems in hierarchical-tree clustering. Acta Informatica, 23:311-323, 1986.

[99] U. Laserson, H. H. Gan, and et al. Searching 2d rna geometries in bacterial genomes. In proceedings of the 12th Annual Symposium on Computational geometry, pages 373-377, 2004.

[100] M. W. L. Lau, K. E. C. Cadieux, and P. J. Unrau. Isolation of fast purine nucleotide synthase ribozymes. Journal of the American Chemical Society, 126:15686-15693, 2004.

[101] S. Le, R. Nussinov, and J. Maizel. Tree graphs of rna secondary structures and their comparisons. Comput. Biomed. Res., 22:461-473, 1989.

[102] J. F. Lee, J. R. Hesselberth, L. A. Meyers, and A. D. Ellington. Aptamer database. Nucleic Acids Research, 32:D95-D100, 2004.

[103] S. Lee, F. Hormozdiari, C. Alkan, and M. Brudno. Modil: detecting small indels from clone-end sequencing with mixtures of distributions. Nature methods, 6:473-474, 2009.

[104] M. Legiewicz, A. Wichlacz, and et al. Antigenomic delta ribozyme variants with mutations in the catalytic core obtained by the in vitro selection method. Nucleic Acids Res, 34(4):1270-1280, 2006. 
[105] S. Levy, G. Sutton, P. Ng, L. Feuk, A. Halpern, B. Walenz, N. Axelrod, J. Huang, E. Kirkness, G. Denisov, Y. Lin, J. MacDonald, A. Pang, M. Shago, T. Stockwell, A. Tsiamouri, V. Bafna, V. Bansal, S. Kravitz, D. Busam, K. Beeson, T. McIntosh, K. Remington, J. Abril, J. Gill, J. Borman, Y. Rogers, M. Frazier, S. Scherer, R. Strausberg, and J. Venter. The diploid genome sequence of an individual human. PLoS biology, 5:e254, 2007.

[106] H. Li and R. Durbin. Fast and accurate short read alignment with burrowswheeler transform. Bioinformatics, 25:1754-1760, 2009.

[107] H. Li, J. Ruan, and R. Durbin. Mapping short dna sequencing reads and calling variants using mapping quality scores. Genome research, 18:1851 - 1858, 2008.

[108] D. M. Lilley, R. M. Clegg, S. Diekmann, N. C.Seeman, E. V. Kitzing, and P. J. Hagerman. A nomenclature of junctions and branchpoints in nucleic acids. Nucleic Acids Res., 23:3363 - 3364, 1995.

[109] K. Liolios, I. Chen, K. Mavromatis, N. Tavernarakis, P. Hugenholtz, V. Markowitz, and N. Kyrpides. The genomes on line database (gold) in 2009: status of genomic and metagenomic projects and their associated metadata. Nucleic acids research, 38:D346-354, 2009.

[110] X. Luo, F. Dehne, and P. Liang. Identification of transposon insertion polymorphisms by computational comparative analysis of next generation personal genome data. In AIP Proceedings of AMMCS, 2011, submitted.

[111] X. Luo, M. McKeague, S. Pitre, M. Dumontier, J. Green, A. Golshani, M. C. DeRosa, and F. Dehne. Computational approaches towards the design of pools for the in vitro selection of complex aptamers. $R N A, 16(11): 2252-2262,2010$.

[112] N. M. Luscombe, D. Greenbaum, and M. Gerstein. What is bioinformatics? a proposed definition and overview of the field. Method Inform Med, 40:346358, 2001.

[113] E. Mardis. Next-generation dna sequencing methods. Annual review of genomics and human genetics, 9:387-402, 2008.

[114] M. Margulies, M. Egholm, W. Altman, S. Attiya, J. Bader, L. Bemben, J. Berka, M. Braverman, Y. Chen, Z. Chen, S. Dewell, L. Du, J. Fierro, X. Gomes, B. Godwin, W. He, S. Helgesen, C. Ho, G. Irzyk, S. Jando, M. Alenquer, T. Jarvie, K. Jirage, J. Kim, J. Knight, J. Lanza, J. Leamon, S. Lefkowitz, L. J. L. K. Lei, M., H. Lu, V. Makhijani, K. McDade, M. McKenna, E. Myers, E. Nickerson, J. Nobile, R. Plant, B. Puc, M. Ronan, G. Roth, G. Sarkis, J. Simons, J. Simpson, M. Srinivasan, K. Tartaro, A. Tomasz, K. Vogt, G. Volkmer, S. Wang, Y. Wang, M. Weiner, P. Yu, B. R.F., and J. Rothberg. Genome sequencing in microfabricated high-density picolitre reactors. Nature, 437:376$380,2005$. 
[115] C. Marshall, A. Noor, J. Vincent, A. Lionel, L. Feuk, J. Skaug, M. Shago, R. Moessner, D. Pinto, Y. Ren, B. Thiruvahindrapduram, A. Fiebig, S. Schreiber, J. Friedman, C. Ketelaars, Y. Vos, C. Ficicioglu, S. Kirkpatrick, R. Nicolson, L. Sloman, A. Summers, C. Gibbons, A. Teebi, D. Chitayat, R. Weksberg, A. Thompson, C. Vardy, V. Crosbie, S. Luscombe, R. Baatjes, L. Zwaigenbaum, W. Roberts, B. Fernandez, P. Szatmari, and S. Scherer. Structural variation of chromosomes in autism spectrum disorder. American journal of human genetics, 82:477 - 488, 2008.

[116] D. H. Mathews, J. Sabina, M. Zuker, and D. H. Turner. Expanded sequence dependence of thermodynamic parameters improves prediction of rna secondary structure. Journal of molecular biology, 288:911 - 940, 1999.

[117] A. Mathuriya, D. A. Bader, C. E. Heitsch, and S. C. Harvey. Gtfold: A scalable multicore code for rna secondary structure prediction. In 24th Annual ACM Symposium on Applied Computing (SAC), pages 981 - 988, 2009.

[118] A. Maxam and W. Gilbert. A new method for sequencing dna. Proceedings of the National Academy of Sciences of the United States of America, 74:560-564, 1977.

[119] S. McCarroll and et al. Common deletion polymorphisms in the human genome. Nature genetics, 38:86-92, 2006.

[120] S. McCarroll, A. Huett, P. Kuballa, S. Chilewski, A. Landry, P. Goyette, M. Zody, J. Hall, S. Brant, J. Cho, R. Duerr, M. Silverberg, K. Taylor, J. Rioux, D. Altshuler, M. Daly, and R. Xavier. Deletion polymorphism upstream of irgm associated with altered irgm expression and crohn's disease. Nature genetics, 40:1107 - 1112, 2008.

[121] B. McCLINTOCK. The origin and behavior of mutable loci in maize. Proc Natl Acad Sci U S A, 36:344 - 355, 1950.

[122] K. McKernan and et al. Sequence and structural variation in a human genome uncovered by short-read, massively parallel ligation sequencing using two-base encoding. Genome research, 19:1527-1541, 2009.

[123] P. Medvedev, M. Stanciu, and M. Brudno. Computational methods for discovering structural variation with next-generation sequencing. Nature methods, 6:S13-20, 2008.

[124] H. Mewes, C. Amid, R. Arnold, D. Frishman, U. Guldener, G. Mannhaupt, M. Munsterkotter, P. Pagel, N. Strack, V. Stumpflen, J. Warfsmann, and A. Ruepp. Mips: analysis and annotation of proteins from whole genomes. Nucleic acids research, pages D41-44, 2004. 
[125] H. W. Mewes, D. Frishman, U. Guldener, G. Mannhaupt, K. Mayer, M. Mokrejs, B. Morgenstern, M. Munsterkotter, S. Rudd, and B. Weil. Mips:a database for genomes and protein sequences. Nucleıc Acrds Res, 30:31-34, 2002.

[126] R. Mills, E. Bennett, R. Iskow, and S. Devine. Which transposable elements are active in the human genome? Trends Genet, 23:183 - 191, 2007.

[127] R. Niedermeier. Invitatıon to Fuxed-Parameter Algorıthms. Oxford University Press, 2006.

[128] NIH. NIH working definition of bioinformatics and computational biology, July 2000. http://www.bisti.nih.gov/docs/CompuBioDef.pdf.

[129] NIH. NHGRI seeks next generation of sequencing technologies, October 2004. http://www.genome.gov/12513210.

[130] S. J. Ohuchi, Y. Ikawa, H. Shiraishi, and T. Inoue. Modular engineering of a group i intron ribozyme. Nucleuc Acıds Res, 30(15):3473-3480, 2002.

[131] S. J. Ohuchi, Y. Ikawa, H. Shiraishi, and T. Inoue. Artificial modules for enhancing rate constants of a group i intron ribozyme without a p4-p6 core element. J. Brol. Chem., 279(1):540-546, 2004.

[132] E. Ostertag, H. Kazazian, and Jr. Biology of mammalian 11 retrotransposons. Annual revıew of genetıcs, 35:501 - 538, 2001.

[133] M. Parisien and F. Major. The mc-fold and mc-sym pipeline infers rna structure from sequence data. Nature, 452:51 - 55, 2008.

[134] J. Pevsner. Bıoınformatıcs and Functıonal Genomıcs. Wiley-Blackwell, 2009.

[135] D. Pinkel and et al. High resolution analysis of dna copy number variation using comparative genomic hybridization to microarrays. Nature genetıcs, 20:207-211, 1998.

[136] M. Pop. Genome assembly reborn: recent computational challenges. Briefings in bioınformatıcs, 10:354-366, 2009.

[137] J. F. Poyatos and L. D. Hurst. How biologically relevant are interaction-based modules in protein networks? Genome Bıol, 5(11):R93, 2004.

[138] A. L. Price, E. Eskin, and P. A. Pevzner. Whole-genome analysis of alu repeat elements reveals complex evolutionary history. Genome Res., 14:2245-2252, 2004.

[139] F. Protti, M. D. da Silva, and J. L. Szwarcfiter. Applying modular decomposition to parameterized bicluster editing. In Proceedings of IWPEC, pages 1 12. Springer LNCS 4169, 2006. 
[140] F. Protti, M. D. da Silva, and J. L. Szwarcfiter. Applying modular decomposition to parameterized cluster editing problems. Theory of Computing Systems, 44(1):91 - 104, 2009.

[141] N. Przulj. Graph theory approaches to protein interaction data analysis. CRC Press, 2004.

[142] C. Reidys, F. W. Huang, J. E. Andersen, R. C. Penner, P. F. Stadler, and M. E. Nebel. Topology and prediction of rna pseudoknots. Bioinformatics, 27:1076-1085, 2011.

[143] E. Rivas and S. R. Eddy. A dynamic programming algorithm for rna structure prediction including pseudoknots. J. Mol. Biol., 285:2053-2068, 1999.

[144] A. W. Rives and T. Galitski. Modular organization of cellular networks. Proc Natl Acad Sci USA, 100(3):12123-12128, 2003.

[145] Y. Rogers and J. Venter. Genomics: massively parallel sequencing. Nature, 437:326-327, 2005.

[146] A. Roy, M. Carroll, D. Kass, S. Nguyen, A. Salem, M. Batzer, and P. Deininger. Recently integrated human alu repeats: finding needles in the haystack. Genetica, 107:149 - 161, 1999 .

[147] P. C. Sabeti, P. J. Unrau, and D. P. Bartel. Accessing rare activities from random rna sequences: the importance of the length of molecules in the starting pool. Chemistry and biology, 4(10):767 - 774, 1997.

[148] F. Sanger, G. Air, B. Barrell, N. Brown, A. Coulson, C. Fiddes, C. Hutchison, P. Slocombe, and M. Smith. Nucleotide sequence of bacteriophage phi x174 dna. Nature, 265:687 - 695, 1977.

[149] F. Sanger, A. Coulson, G. Hong, D. Hill, and G. Petersen. Nucleotide sequence of bacteriophage lambda dna. Journal of molecular biology, 162:729-773, 1982.

[150] F. Sanger, S. Nicklen, and A. Coulson. Dna sequencing with chain-terminating inhibitors. Proceedings of the National Academy of Sciences of the United States of America, 74:5463-5467, 1977.

[151] S. Schuster. Next-generation sequencing transforms today's biology. Nature methods, 5:16-18, 2008.

[152] H. Schwalbe, J. Buck, B. Furtig, J. Noeske, and J. Wohnert. Structures of rna switches: insight into molecular recognition and tertiary structure. Angewandte Chemie International ed. in English, 46(8):1212 - 1219, 2007. 
[153] W. G. Scott, J. B. Murray, J. R. Arnold, B. L. Stoddard, and A. Klug. Capturing the structure of a catalytic rna intermediate: the hammerhead ribozyme. Science, 274:2065 - 2069, 1996.

[154] J. Sebat and et al. Large-scale copy number polymorphism in the human genome. Science, 305:525-528, 2004.

[155] S. K. Sen, K. Han, J. Wang, J. Lee, H. Wang, P. A. Callinan, M. Dyer, R. Cordaux, P. Liang, and M. A. Batzer. Human genomic deletions mediated by recombination between alu elements. Am J Hum Genet, 79:41 - 53, 2006.

[156] A. Serganov, Y. R. Yuan, O. Pikovskaya, A. Polonskaia, L. Malinina, A. T. Phan, and et al. Structural basis for discriminative regulation of gene expression by adenine- and guanine-sensing mrnas. Chem. Biol., 11:1729-1741, 2004.

[157] D. Shangguan, Y. Li, and et al. Aptamers evolved from live cells as effective molecular probes for cancer study. PNAS, 103:11838-11843, 2006.

[158] B. Shapiro and K. Zhang. Comparing multiple rna secondary structures using tree comparisons. Comput. Appl. Biosci., 6:309 318, 1990.

[159] R. Sharan and R. Shamir. Algorithmic approaches to clustering gene expression data. In T. Jiang, Y. Xu, and M. Q. Zhang, editors, Current Topics in Computational Molecular Biology, pages 269 - 300. The MIT Press, 2002.

[160] A. Sharp, Z. Cheng, and E. Eichler. Structural variation of the human genome. Annu. Rev. Genom. Human Genet., 7:407-442, 2006.

[161] A. Sharp and et al. Segmental duplications and copy-number variation in the human genome. Am. J. Hum. Genet., 77:78-88, 2004.

[162] J. Shendure and H. Ji. Next-generation dna sequencing. Nature biotechnology, 26:1135-1145, 2008.

[163] A. F. Smit. Interspersed repeats and other mementos of transposable elements in mammalian genomes. Curr. Opin. Genet. Dev., 9:657 - 663, 1999.

[164] G. A. Soukup and R. R. Breaker. Nucleic acid molecular switches. Trends Biotechnol, 17(12):469-476, 1999.

[165] V. Spirin and L. A. Mirny. Protein complexes and functional modules in molecular networks. Proc. Natl Acad. Sci., 100:12123-12128, 2003.

[166] R. Staden. A strategy of dna sequencing employing computer programs. Nucleic acids research, 6:2601-2610, 1979. 
[167] C. Stark, B. J. Breitkreutz, T. Reguly, L. Boucher, A. Breitkreutz, and M. Tyers. Biogrid: a general repository for interaction datasets. Nuclevc Acıds Res, 34(Database issue):D535 - 539, 2006.

[168] F. Stuhlmann and A. Jaschke. Characterization of an rna active site: Interactions between a diels-alderase ribozyme and its substrates and products. $J$. Am. Chem. Soc., 124:3238-3244, 2002.

[169] F. Tang, C. Barbacioru, Y. Wang, E. Nordman, C. Lee, N. Xu, X. Wang, J. Bodeau, B. Tuch, A. Siddiqui, K. Lao, and M. Surani. mrna-seq wholetranscriptome analysis of a single cell. Nature methods, 6:377-382, 2009.

[170] V. Thodima, M. Pirooznia, and Y. Deng. Riboaptdb: A comprehensive database of ribozymes and aptamers. BMC Broinformatzcs, Suppl 2:S6, 2006.

[171] C. Tuerk and L. Gold. Systematic evolution of ligands by exponential enrichment: Rna ligands to bacteriophage $\mathrm{t} 4$ dna polymerase. Scıence, 249:505-510, 1990.

[172] E. Tuzun, A. Sharp, J. Bailey, R. Kaul, V. Morrison, L. Pertz, E. Haugen, H. Hayden, D. Albertson, D. Pinkel, M. Olson, and E. Eichler. Fine-scale structural variation of the human genome. Nature genetzcs, 37:727 - 732, 2005.

[173] A. Valouev, J. Ichikawa, T. Tonthat, J. Stuart, S. Ranade, H. Peckham, K. Zeng, J. Malek, G. Costa, K. McKernan, A. Sidow, A. Fire, and S. Johnson. A highresolution, nucleosome position map of c. elegans reveals a lack of universal sequence-dictated positioning. Genome research, 18:1051-1063, 2008.

[174] J. Venter, M. Adams, E. Myers, P. Li, R. Mural, G. Sutton, and et al. The sequence of the human genome. Scrence, 291:1304-1351, 1995.

[175] K. Voelkerding, S. Dames, and J. Durtschi. Next-generation sequencing: from basic research to diagnostics. Clinical chemistry, 55:641-658, 2009.

[176] H. Wang, J. Xing, D. Grover, D. Hedges, K. Han, W. J.A., and B. M.A. Sva elements: a hominid-specific retroposon family. J. Mol. Bıol, 354:994-1007, 2005.

[177] J. Wang, L. Song, M. Gonder, S. Azrak, D. Ray, M. Batzer, S. Tishkoff, and P. Liang. Whole genome computational comparative genomics: A fruitful approach for ascertaining alu insertion polymorphisms. Gene, 365:11 - 20, 2006.

[178] J. Wang, L. Song, D. Grover, S. Azrak, M. Batzer, and P. Liang. dbrip: a highly integrated database of retrotransposon insertion polymorphisms in humans. Human mutatıon, 27:323 - 329, 2006. 
[179] J. Wang, W. Wang, R. Li, Y. Li, G. Tian, L. Goodman, W. Fan, J. Zhang, J. Li, J. Zhang, Y. Guo, B. Feng, H. Li, Y. Lu, X. Fang, H. Liang, Z. Du, D. Li, Y. Zhao, Y. Hu, Z. Yang, H. Zheng, I. Hellmann, M. Inouye, J. Pool, X. Yi, J. Zhao, J. Duan, Y. Zhou, J. Qin, L. Ma, G. Li, Z. Yang, G. Zhang, B. Yang, C. Yu, F. Liang, W. Li, S. Li, D. Li, P. Ni, J. Ruan, Q. Li, H. Zhu, D. Liu, Z. Lu, N. Li, G. Guo, J. Zhang, J. Ye, L. Fang, Q. Hao, Q. Chen, Y. Liang, Y. Su, A. San, C. Ping, S. Yang, F. Chen, L. Li, K. Zhou, H. Zheng, Y. Ren, L. Yang, Y. Gao, G. Yang, Z. Li, X. Feng, K. Kristiansen, G. Wong, R. Nielsen, R. Durbin, L. Bolund, X. Zhang, S. Li, H. Yang, and J. Wang. The diploid genome sequence of an asian individual. Nature, 456:60 - 65, 2008.

[180] D. Wheeler, M. Srinivasan, M. Egholm, Y. Shen, L. Chen, A. McGuire, W. He, Y. Chen, V. Makhijani, G. Roth, X. Gomes, K. Tartaro, F. Niazi, C. Turcotte, G. Irzyk, J. Lupski, C. Chinault, X. Song, Y. Liu, Y. Yuan, L. Nazareth, X. Qin, D. Muzny, M. Margulies, G. Weinstock, R. Gibbs, and J. Rothberg. The complete genome of an individual by massively parallel dna sequencing. Nature, 452:872-876, 2008.

[181] D. S. Wilson and J. W. Szostak. In vitro selection of functional nucleic acids. Annu Rev Biochem, 68:611-647, 1999.

[182] T. J. Wilson, M. Nahas, T. Ha, and D. M. Lilley. Folding and catalysis of the hairpin ribozyme. Biochem. Soc. Trans., 33:461 - 465, 2005.

[183] D. Witherspoon, J. Xing, Y. Zhang, W. Watkins, M. Batzer, and L. Jorde. Mobile element scanning (me-scan) by targeted high-throughput sequencing. $B M C$ genomics, 11:410, 2008.

[184] J. Xing, H. Wang, V. P. Belancio, R. Cordaux, P. L. Deininger, and M. A. Batzer. Emergence of primate genes by retrotransposon-mediated sequence transduction. Proc Natl Acad Sci U S A, 103:17608 - 17613, 2006.

[185] J. Xing, Y. Zhang, K. Han, A. Salem, S. Sen, C. Huff, Q. Zhou, E. Kirkness, M. Levy, S. andDurbin10 Batzer, and L. Jorde. Mobile elements create structural variation: analysis of a complete human genome. Genome research, 19:1516 - 1526, 2009.

[186] K. Ye, M. H. Schulz, Q. Long, R. Apweiler, and Z. Ning. Pindel: a pattern growth approach to detect breakpoints of large deletions and medium sized insertions from paired-end short reads. Bioinformatics, 25:2865-2871, 2009.

[187] W. Yoshioka, Y. Ikawa, and et al. Generation of a catalytic module on a selffolding rna. RNA, 10(12):1900-1906, 2004.

[188] H. Yu, A. Paccanaro, V. Trifonov, and M. Gerstein. Predicting interactions in protein networks by completing defective cliques. Bioinformatics, 22:823-829, 2006. 
[189] Y. Zhang, F. N. Abu-Khzam, N. E. Baldwin, E. J. Chesler, M. A. Langston, and N. F. Samatova. Genome-scale computational approaches to memory-intensive applications in systems biology. In Proceedings, Supercomputing, Seattle, Washington, 2005.

[190] S. P. Zinnen, K. Domenico, and et al. Selection, design, and characterization of a new potentially therapeutic ribozyme. $R N A, 8(2): 214-228,2002$.

[191] M. Zuker. Mfold web server for nucleic acid folding and hybridization prediction. Nucleic acids research, 31:3406 - 3415, 2003.

[192] M. Zuker and P. Stiegler. Optimal computer folding of large rna sequences using thermodynamic and auxiliary information. Nucl Acid Res, 9:133-148, 1981. 